\title{
WestVirginiaUniversity
}

THE RESEARCH REPOSITORY @ WVU

Graduate Theses, Dissertations, and Problem Reports

2004

\section{Biologically inspired evolutionary temporal neural circuits}

\author{
Reza Derakhshani \\ West Virginia University
}

Follow this and additional works at: https://researchrepository.wvu.edu/etd

\section{Recommended Citation}

Derakhshani, Reza, "Biologically inspired evolutionary temporal neural circuits" (2004). Graduate Theses, Dissertations, and Problem Reports. 2110.

https://researchrepository.wvu.edu/etd/2110

This Dissertation is protected by copyright and/or related rights. It has been brought to you by the The Research Repository @ WVU with permission from the rights-holder(s). You are free to use this Dissertation in any way that is permitted by the copyright and related rights legislation that applies to your use. For other uses you must obtain permission from the rights-holder(s) directly, unless additional rights are indicated by a Creative Commons license in the record and/ or on the work itself. This Dissertation has been accepted for inclusion in WVU Graduate Theses, Dissertations, and Problem Reports collection by an authorized administrator of The Research Repository @ WVU.

For more information, please contact researchrepository@mail.wvu.edu. 


\title{
Biologically Inspired Evolutionary Temporal Neural Circuits
}

\author{
by \\ Reza Derakhshani \\ Dissertation submitted to the \\ College of Engineering and Mineral Resources \\ at West Virginia University \\ in partial fulfillment of the requirements \\ for the degree of \\ Doctor of Philosophy \\ in \\ Computer Engineering \\ Approved by \\ Stephanie Schuckers, Ph.D., Committee Chairperson \\ Bojan Cukic, Ph.D. \\ Lawrence Hornak, Ph.D. \\ Mark Jerabek, Ph.D. \\ George Spirou, Ph.D.
}

Lane Department of Computer Science and Electrical Engineering

$$
\text { Morgantown, West Virginia }
$$

2004

Keywords: Artificial Neural Networks, Time Delay Neural Networks, Evolving Neural Networks, Evolutionary Algorithms, Sequence Analysis, Intelligent Signal Processing, Pattern Recognition

Copyright 2004 Reza Derakhshani 


\section{Abstract \\ Biologically Inspired Evolutionary Temporal Neural Circuits by Reza Derakhshani}

Biological neural networks have always motivated creation of new artificial neural networks, and in this case a new autonomous temporal neural network system. Among the more challenging problems of temporal neural networks are the design and incorporation of short and long-term memories as well as the choice of network topology and training mechanism. In general, delayed copies of network signals can form shortterm memory (STM), providing a limited temporal history of events similar to FIR filters, whereas the synaptic connection strengths as well as delayed feedback loops (IIR circuits) can constitute longer-term memories (LTM). This dissertation introduces a new general evolutionary temporal neural network framework (GETnet) through automatic design of arbitrary neural networks with STM and LTM. GETnet is a step towards realization of general intelligent systems that need minimum or no human intervention and can be applied to a broad range of problems. GETnet utilizes nonlinear moving average/ autoregressive nodes and sub-circuits that are trained by enhanced gradient descent and evolutionary search in terms of architecture, synaptic delay, and synaptic weight spaces. The mixture of Lamarckian and Darwinian evolutionary mechanisms facilitates the Baldwin effect and speeds up the hybrid training. The ability to evolve arbitrary adaptive time-delay connections enables GETnet to find novel answers to many classification and system identification tasks expressed in the general form of desired multidimensional input and output signals. Simulations using Mackey-Glass chaotic time series and fingerprint perspiration-induced temporal variations are given to demonstrate the above stated capabilities of GETnet. 


\section{DEDICATION}

To Dr. Michael Henry, the man who introduced me to the amazing world of Neurocomputing. 


\section{ACKNOWLEDGEMENTS}

I want to thank my advisor, Dr. Stephanie Schuckers, for her help, mentoring, encouragement, and valuable advice. I would also like to extend my appreciation and gratitude to my other committee members, Dr. Bojan Cukic, Dr. Lawrence Hornak, Dr. Mark Jerabek, and Dr. George Spirou, for their guidance, support, and patience. I want to thank Mr. Ray Lane for the generous support he provided me through the Lane fellowship. I am also thankful to my colleagues at the Biomedical Signal Analysis Lab, West Virginia University, especially Pisut Raphisak, Simona Crihalmeanu, and Rohin Govindarajn for their help and cooperation.

Last but not the least, I want to thank my family: my wife Maria, my mother Shahzad, my father Khalil, and my sisters Taraneh, Hanieh, and Sara, and my uncle Mohammad, for their continuous love and support. Without your sacrifices I would never have been where I am today. Thank you all. 


\section{TABLE OF CONTENTS}

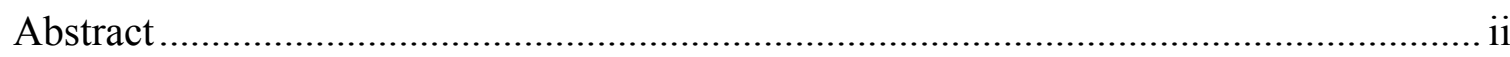

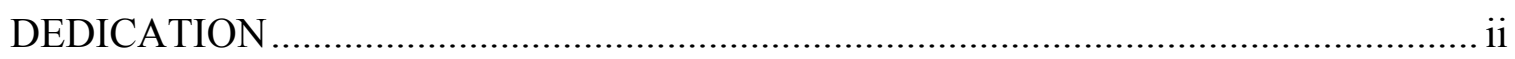

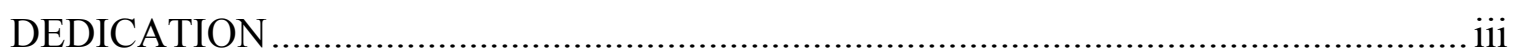

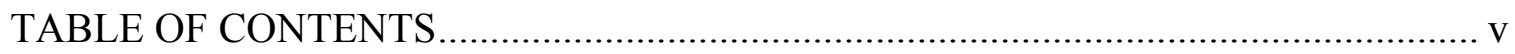

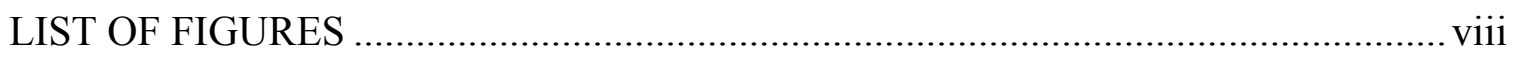

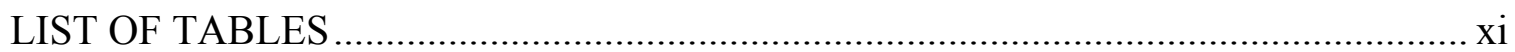

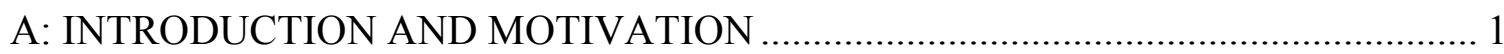

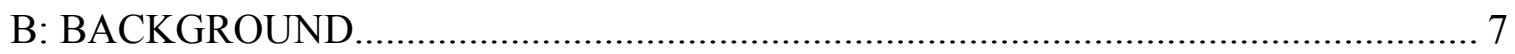

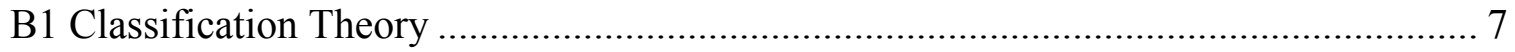

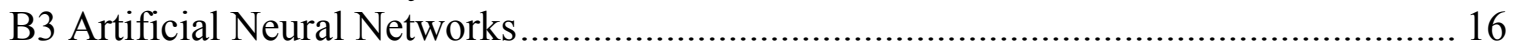

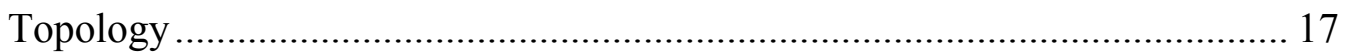

Performance Measures................................................................................ 17

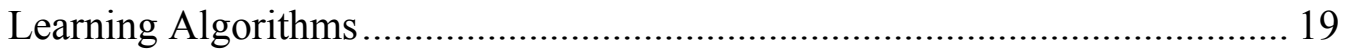

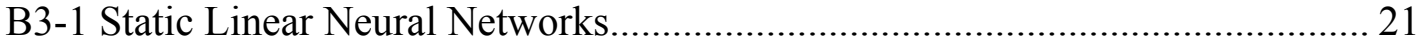

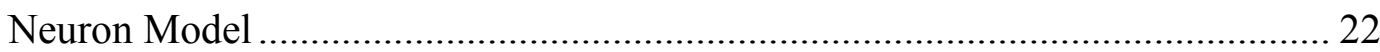

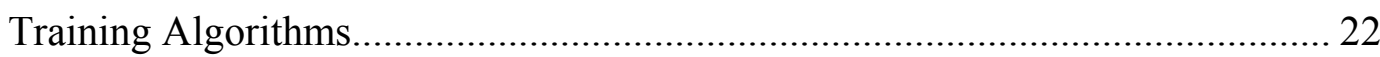

First Order Algorithms: LMS Method ........................................................... 22

Second Order Algorithm: Newton's Method..................................................... 26

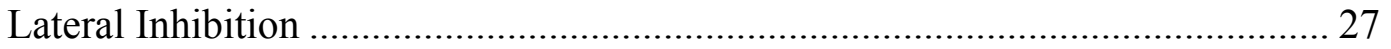

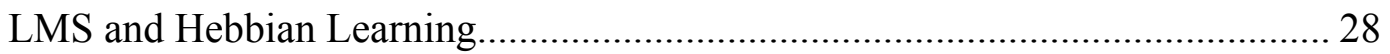

B3-2 Dynamic Linear Neural Networks ............................................................ 30

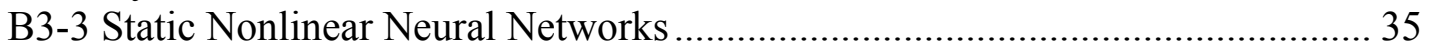

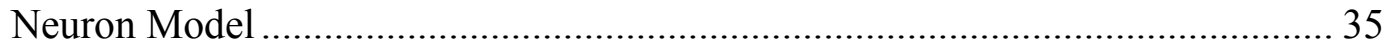

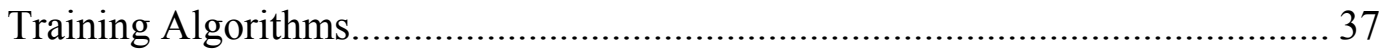

Multi-Layer Networks ...................................................................................... 37

Computation of Gradients in Ordered Networks ............................................... 41

Improving Backpropagation Learning .............................................................. 49

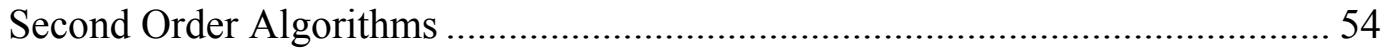

Improving Backpropagation For Unseen Data ................................................ 58

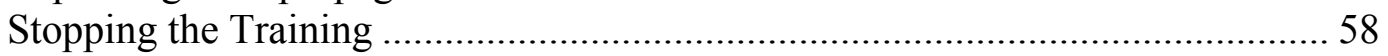

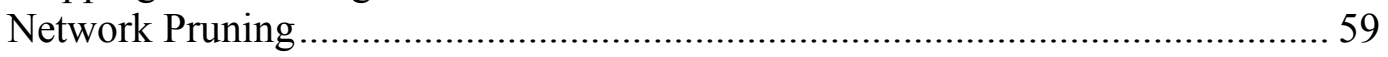

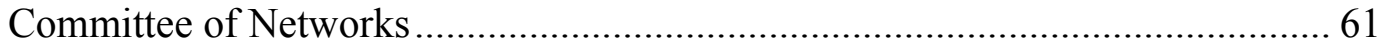

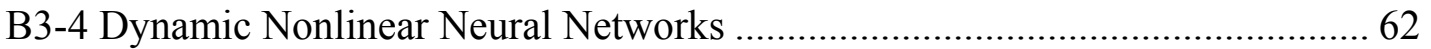

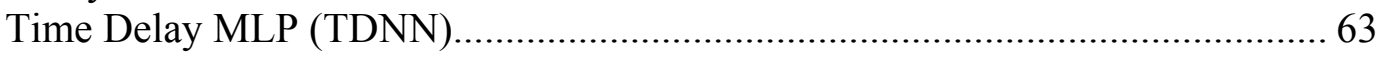

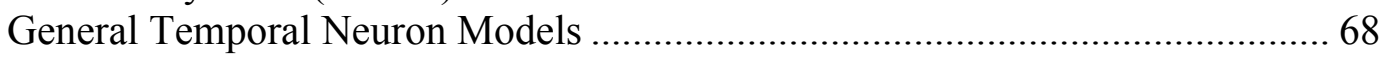

Training Recurrent Neural Networks................................................................ 71

Network Energy, Hopfield and Boltzmann Neural Networks ................................ 75

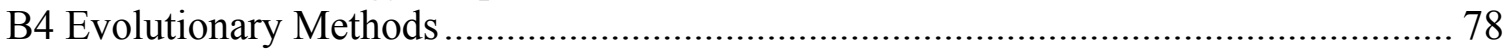


B4-1 A Review of Evolutionary Computing ...................................................... 78

Evolutionary Algorithms (EA), General Concepts ………................................. 78

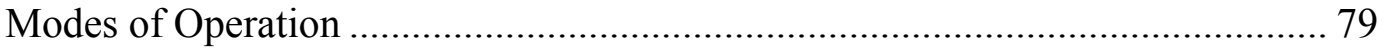

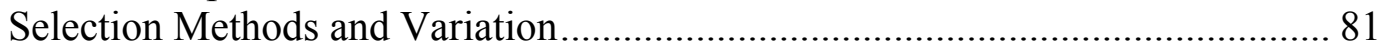

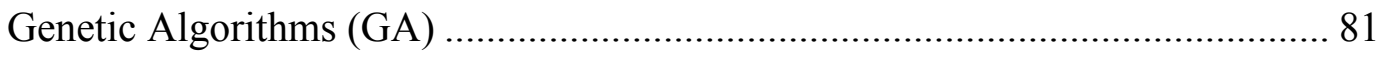

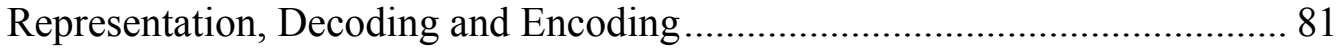

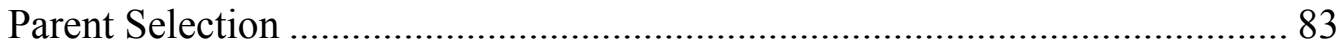

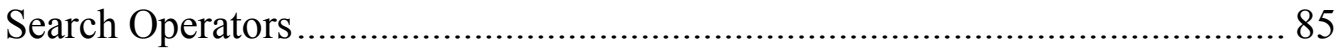

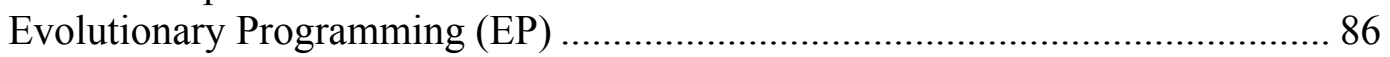

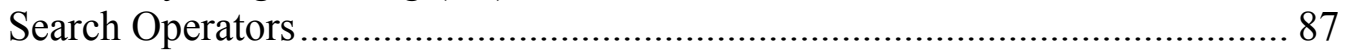

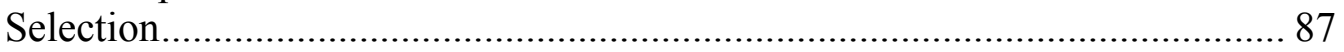

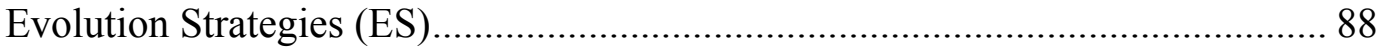

B4-2 Application of Evolutionary Methods to Artificial Neural Networks ............. 91

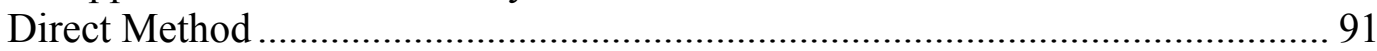

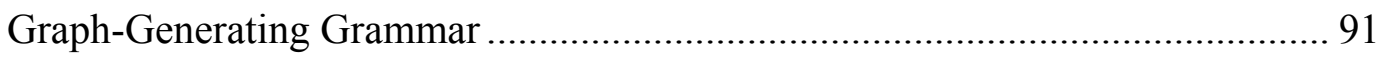

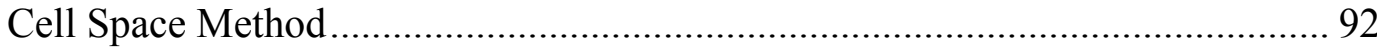

Co-Evolution of Architecture and Parameters ...................................................... 93

C: SUGGESTED GENERAL EVOLUTIONARY TEMPORAL NEURAL NETWORK

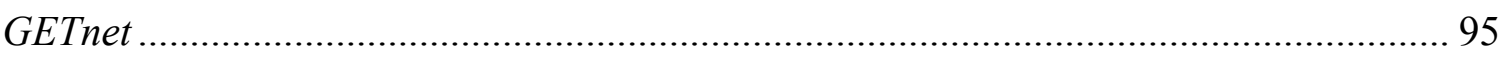

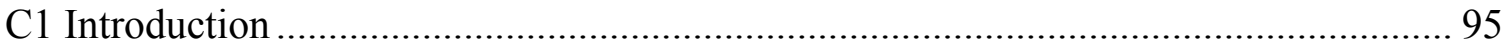

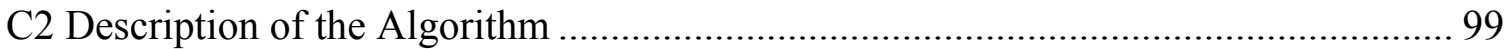

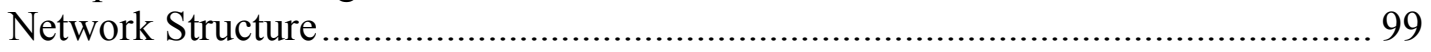

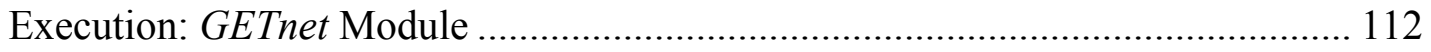

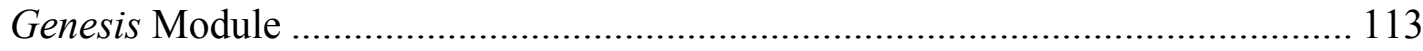

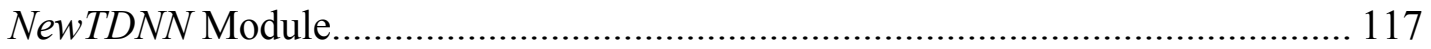

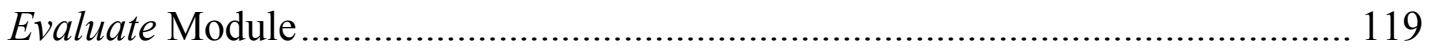

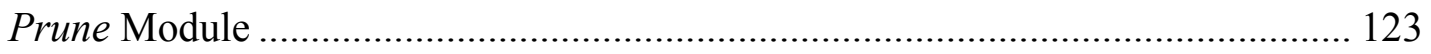

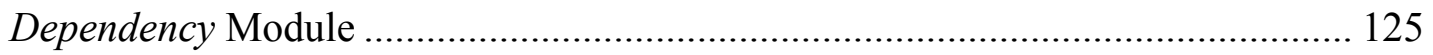

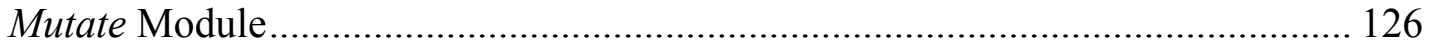

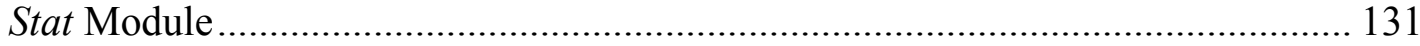

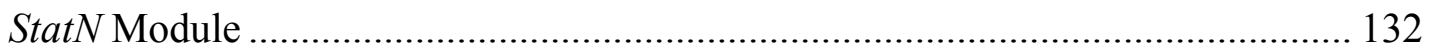

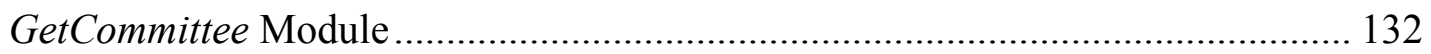

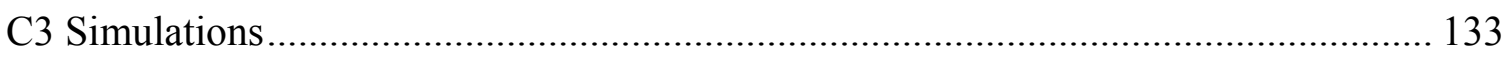

Mackey-Glass Chaotic Series 1 ...................................................................... 133

Problem Description ................................................................................. 133

Data and Simulation Settings, 6-Step Prediction ................................................. 134

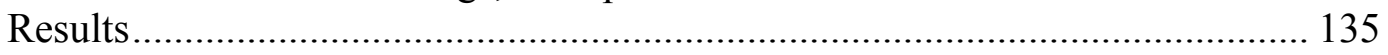

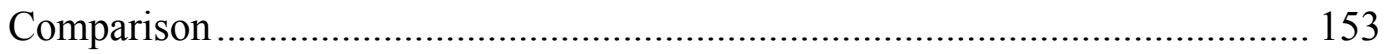

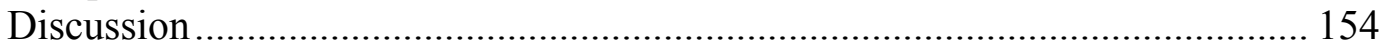

Mackey-Glass Chaotic Series 2 ……………………................................... 157

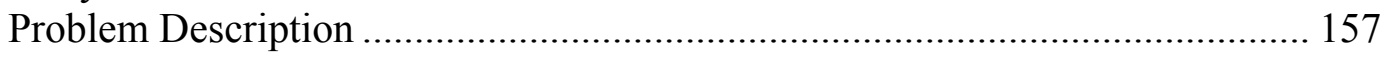

Data and Simulation Settings, 36-Step Prediction............................................... 157

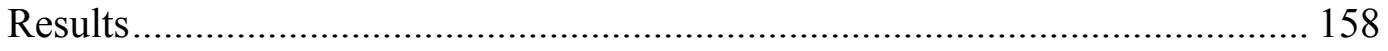

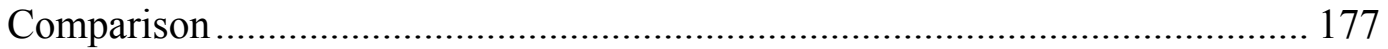




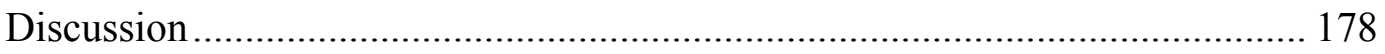

Fingerprint Perspiration Sequence Detection .................................................. 181

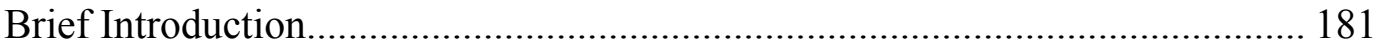

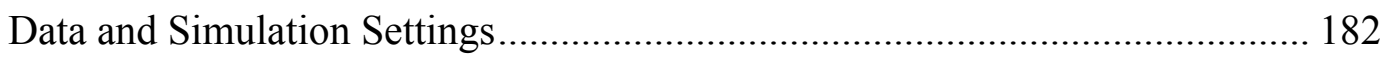

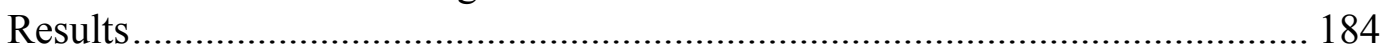

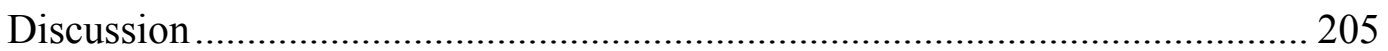

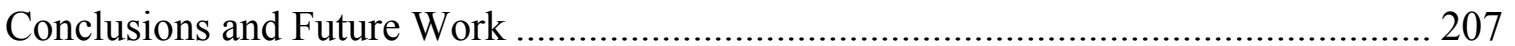

Appendix A: More on Gradient Conjugate Methods..................................................... 213

Appendix B: Nguyen-Widrow Weight Initialization Algorithm .................................. 214

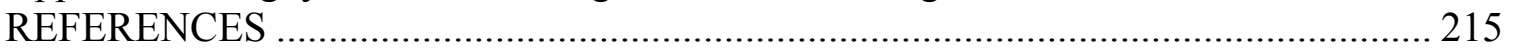

CURRICULUM VITAE 226 


\section{LIST OF FIGURES}

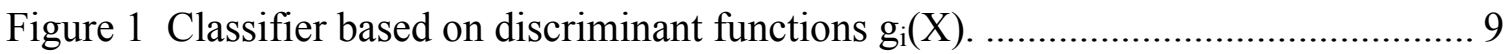

Figure 2 A kernel-based classifier. ....................................................................... 10

Figure 3 Plot of $\mathrm{P}_{\mathrm{N}}(\mathrm{M})$ demonstrates Cover's Theorem........................................... 12

Figure 4 Solid curve shows fitting a quadratic to 4 points (not enough degrees of freedom, model bias). Dashed curve shows fitting a $6^{\text {th }}$ order curve (extra degrees of

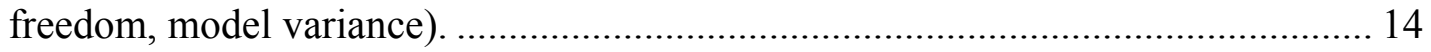

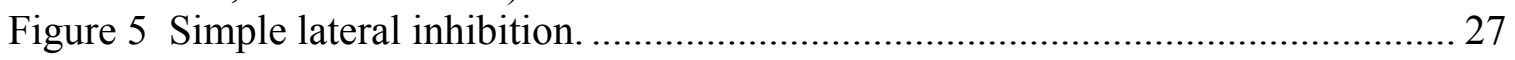

Figure 6 A moving-average linear neuron............................................................ 31

Figure 7 In modified Mcculloch-Pitts neurons class boundary depends on the weight

ratios whereas the transition band depends on the actual weight values. .................. 36

Figure 8 MLPs can create arbitrary convex decision surfaces. .................................... 38

Figure 9 Node notations used in multiple hidden layer MLP back-propagation............. 40

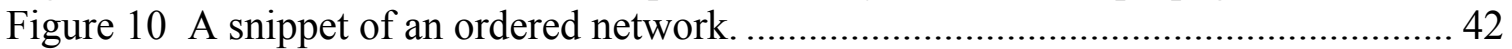

Figure 11 The problem of choosing the right number of hidden units. ......................... 48

Figure 13 Derivative of the sigmoid function has a maximum of 0.25 at the origin....... 52

Figure 14 The network should stop early at point A for optimum overall performance on both the training (solid curve) and cross-validation data (dashed curve) and retain its

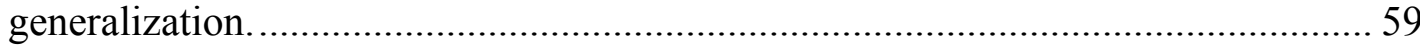

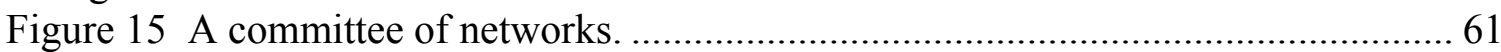

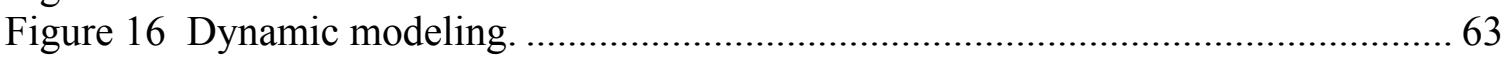

Figure 17 A focused time delay multilayer Perceptron. ............................................. 64

Figure 18 A delay line memory (left) vs. a recurrent or context memory (right)........... 65

Figure 19 Gamma memory (left) and its recurrent context element (right). ................. 65

Figure 20 Jordan temporal network (left) vs. Elman temporal network (right). Bold lines

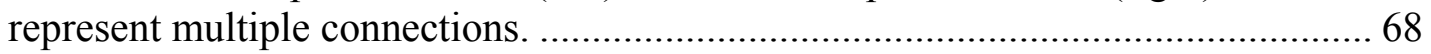

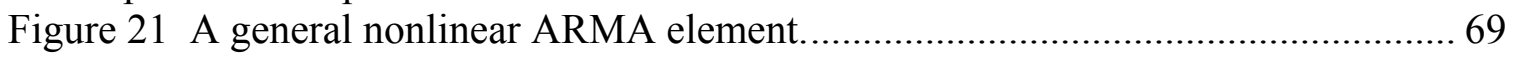

Figure 22 Linear ordering selection probability for a population of $\mu=100$ and $\beta=1.2$

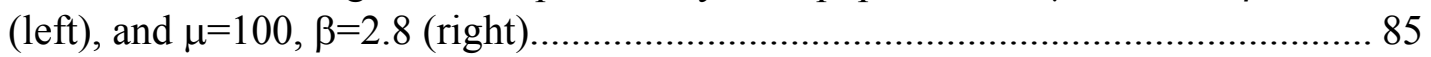

Figure 23 A network resulted from Nolfi and Parisi cell spacing encoding................... 93

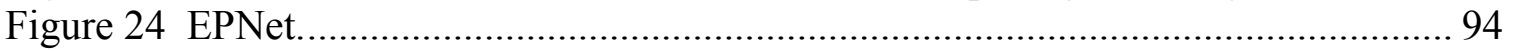

Figure 25 GETnet's flow and organization. The names of actual main modules are italicized, and product of each stage appears after the colon. Secondary helper modules Stat and StatN are not shown for simplicity........................................... 98

Figure 26 A sample network such as the ones generated by the Genesis module.......... 99

Figure 27 A hypothetical performance surface in a 2-D weight space. Ellipsoids show 2 different evolved stochastic search regions around deterministic optima marked with

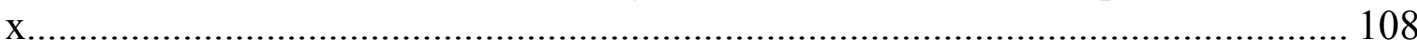

Figure 28 Best evolved network for MG17 six-step prediction. Each line represents a delayed synaptic connection between one input and two layer nodes................... 141

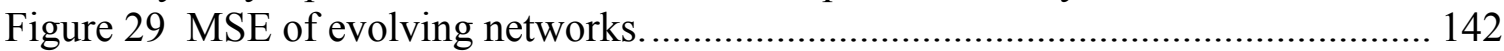

Figure 30 Histogram of the MSEs of the best networks through 203 generations........ 142

Figure 31 Size of evolving networks. ................................................................ 143

viii 
Figure 32 Training data, best evolved network. ………………............................... 143

Figure 33 Training data, magnified section, best evolved network............................ 144

Figure 34 Best evolved network, training error...................................................... 144

Figure 35 Best evolved network: training performance correlation............................. 145

Figure 36 Best evolved network, training data Fourier transform magnitude plots. ..... 145

Figure 37 Training data, committee of last generation networks. ................................ 146

Figure 38 Training data, magnified section for network committee. ............................ 146

Figure 39 Network committee, training error........................................................... 147

Figure 40 Network committee: training performance correlation............................... 147

Figure 41 Network committee, training data Fourier transform magnitude plots......... 148

Figure 42 Test data, best evolved network. ........................................................... 148

Figure 43 Test set performance, magnified. .......................................................... 149

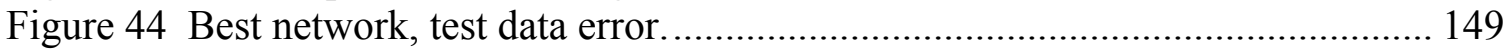

Figure 45 Best evolved network, test set performance correlation................................ 150

Figure 46 Best evolved network, test data Fourier transform magnitude plots............. 150

Figure 47 Test data, committee of last generation networks. ........................................ 151

Figure 48 Test set performance, magnified section for network committee. ………..... 151

Figure 49 Network committee, test data error. ......................................................... 152

Figure 50 Network committee, test data performance correlation. ............................... 152

Figure 51 Network committee, test data Fourier transform magnitude plots................ 153

Figure 52 Best evolved network for MG17 thirty six-step prediction. There is a 30-line delayed synaptic connection between the input and the layer nodes....................... 165

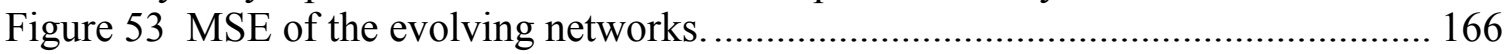

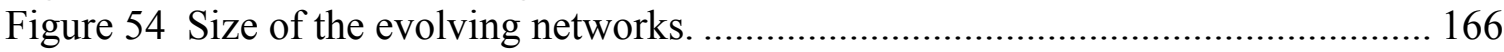

Figure 55 Training data, best evolved network. ………………………………..... 167

Figure 56 Training, magnified section for best evolved network................................ 167

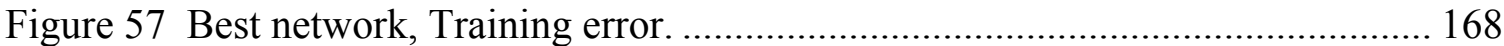

Figure 58 Best evolved network, training performance correlation. ............................ 168

Figure 59 Best evolved network, training data Fourier transform magnitude plots. .... 169

Figure 60 Training data, committee of last generation networks. ................................ 169

Figure 61 Training, magnified section for the network committee............................. 170

Figure 62 Network committee, training error......................................................... 170

Figure 63 Network committee, training performance correlation. ................................ 171

Figure 64 Network committee, training data Fourier transform magnitude plots. ......... 171

Figure 65 Test data, best evolved network. ........................................................ 172

Figure 66 Test set performance, magnified section from the best evolved network. .... 172

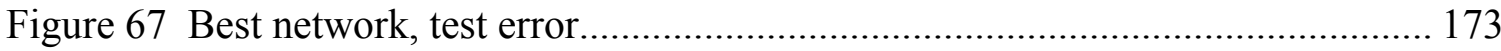

Figure 68 Best evolved network, test set performance correlation............................... 173

Figure 69 Best evolved network, test data Fourier transform magnitude plots............. 174

Figure 70 Test data, committee of last generation networks. ........................................ 174

Figure 71 Test set performance, magnified section from the network committee. ....... 175

Figure 72 Network committee, test data error........................................................ 175

Figure 73 Network committee, test data performance correlation. ............................... 176

Figure 77 Network committee, test data Fourier transform magnitude plots................ 176 
Figure 78 Perspiration-based fingerprint liveness detection. Top and from left to right: temporal progression of fingerprints. Bottom: conversion of ridge gray levels to signals.

Figure 79 Best evolved network for fingerprint liveness detection. Note the novel structure, delayed weight bus widths, and multiple feedback loops..................... 196

Figure 80 ROC curve for the 30 point test data................................................... 198

Figure 81 Training data. Red: first capture signal, blue: last capture signal. Green high: live signals, green low: nonliving signals......................................................... 199

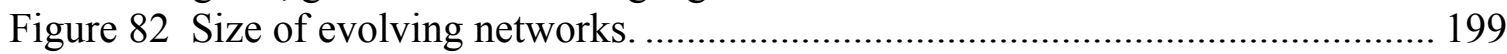

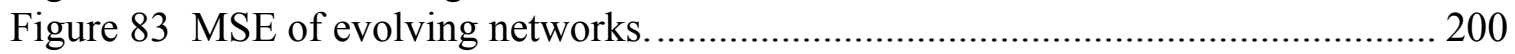

Figure 84 Training output, best evolved network................................................ 200

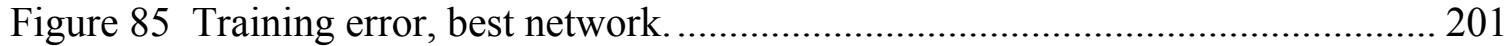

Figure 86 Training data, committee of last generation networks. ................................ 201

Figure 87 Sample live test data output, best evolved network. ................................ 202

Figure 88 Sample live test data output, committee of last generation networks.......... 202

Figure 89 Sample cadaver test data output, best evolved network.............................. 203

Figure 90 Sample cadaver test data output, committee of last generation networks..... 203

Figure 91 Sample spoof test data output, best evolved network................................ 204

Figure 92 Sample spoof test data output, committee of last generation networks. ....... 204 


\section{LIST OF TABLES}

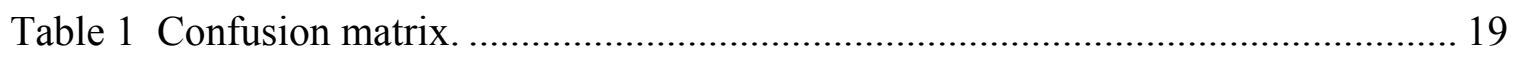

Table 2 Test outputs for live subjects. Incorrect classifications are italicized............... 197

Table 3 Test outputs for cadaver subjects. Incorrect classifications are italicized........ 197

Table 4 Test outputs for spoof subjects. Incorrect classifications are italicized............ 197

Table 5 Confusion matrix for the test data. Threshold for network output is set at zero. 


\section{A: INTRODUCTION AND MOTIVATION}

Asim Roy ${ }^{1}$ mentioned the extensive and tedious steps for producing an effective neural network as the major criticism for this otherwise very powerful paradigm. The need for human experts to constantly intervene in the design and training processes of a neural network is also known as the "baby sitting" problem of the artificial neural networks, which according to Roy has degraded them to "just another way of solving a problem". He also mentions that the most significant, and currently absent, biological resemblance of the artificial neural networks to real brains should be automatic learning, and so suggests automating the learning and design processes to alleviate current practical problems of artificial neural networks. However, this automation involves fundamental issues that are considered open and unanswered. Addressing the baby-sitting problem is the key to solving the current paradoxical situation of needing human experts with vast knowledge to develop a much more restricted intelligent system. For instance, classical neural networks need extensive human expertise to custom design each network to the domain of the problem at hand. This matter becomes more exasperating when even the experts do not readily know what type of neural network system to use.

Addressing this problem is more crucial for the temporal systems. Organisms model and analyze the external world in their minds through the information that they receive from their sensory inputs as a stream of multidimensional temporal signals. In biological brains, the temporal association of synaptic inputs activates cellular mechanisms that underlie such diverse brain processes as learning, memory and coincidence detection for sound localization. Temporal factors can be built into real neural assemblies through repeating units of cellular architecture as are most easily recognized in cortical territories, and tapped delays via branches of axons traversing the entire structure $2,3,4,5$. 
In artificial neural networks, finding the right structure and adaptation algorithm for temporal systems is hard. There are no analytical methods to ensure the quality and capabilities of an arbitrary topology. For instance, in the case of short-term memories implemented with input delay lines, what should be the depth of the delay line? Generally speaking, the size of the feature space for time signals cannot be analytically determined. The same problem exists for implementation of long-term memory structures such as Gamma memories ${ }^{6}$.

Nature has found answers to the above-mentioned problems through genetics and evolution. Biological evidence supports the role of genetics in both anatomy and behavior of the brain. It has been known that learning and memory are related to synaptic architecture and transmission strength ${ }^{7,8,9,10,11,12}$. Genes seem to have a direct role in brain architecture and its learning and memory functions. Studies on artificially mutated Drosophila show definite changes in individual functional components of learning and memory such as loss of short-term memory ${ }^{13,14}$ which result from specific genes' mutations. Some of these learning mutants show no sign of anatomical abnormalities in their brain, while some display obvious neural architecture deformations ${ }^{15,16}$. It has also been shown that synaptic development in Drosophila shares features with higher mammals ${ }^{17,18}$. Thus one can find biological evidence in favor of the application of evolutionary and genetic algorithms to the design of artificial neural circuits.

Based on the above, this dissertation explores a new framework for a unified approach to temporal signal feature extraction, feature selection, and functional approximation. Evolutionary algorithms are applied to determine the design of a temporal neural network for each application, including both the general structure and the specific weights and delays within the structure. The suggested general evolutionary temporal neural networks or GETnet finds the topology, size, connection sparsity, distributed memory depth and structure, synaptic connection strengths, and description complexity of the sought neural network through a unique hybrid system of deterministic and stochastic searches in weight, delay, and architecture spaces. GETnet evolves a general 
class of nonlinear recurrent neural networks (RNN) with distributed delay structures. RNNs can represent arbitrary dynamic systems ${ }^{19,20}$ and are at least as powerful as Turing machines ${ }^{21}$. GETnet also introduces a novel and pragmatic regularization mechanism in order to achieve minimum description length (MDL) solutions to address the biasvariance dilemma and achieve better generalization with smaller data sets.

The following paragraphs summarize the GETnet's algorithm. First, GETnet's algorithm (figure 25) randomly generates a population of temporal neural networks, with single or multidimensional training sequences as input and outputs. Each neuron in a network is connected either to itself or to other neurons with single or multiple branches, each with a specific weight and delay. These connections can be either feed forward or recurrent. Minimum trivial heuristics are used to ensure functionality, such as each network and its nodes should have their input(s) and output(s) connected to somewhere, and that zero-delay loops should be avoided.

Once functionality is checked, each neural network is trained partially on a training dataset. The training in this phase is partial because the gradient descent time is limited to favor more compact networks. This race against time is adjusted in each generation to achieve a functioning minimum description length (temporal MDL) that ensures fastest performance on the hosting hardware. After the networks are trained, adaptive pruning reduces the size of evolving networks. The products of aggressive network minimization through the novel temporal MDL and pruning, as well as fitness scores that are based on unseen validation data, are compact evolved neural networks with minimum variance resulting in excellent generalization capabilities.

Next, the fitness of each individual, pruned neural network in a generation is calculated as the inverse of its mean squared error after partial training. The best networks are chosen based on the fitness function using a roulette wheel form of selection to parent the next generation.

The parents are then mutated in the simulated evolutionary process to form the offspring. Evolution continues until the required precision or maximum time is reached. Mutation is performed for three categories of variables: (1) strategy variables, 
(2) branches (including delays), connections and nodes, and (3) network weights. First mutations of strategy variables, described in section $\mathrm{C}$, define the overall characteristics of the evolution process. Second, additive or subtractive mutations on branch, connection, and node levels are performed. When a structural element is to be added, GETnet tries to follow the overall network pattern to make the augmentation seamless. During the deletion process, chained dependencies are taken into account to calculate the overall effect and avoid disruptive deletions such as removing a network's output path if possible. These smooth mutations reduce the noise in evolutionary assessment of evolving parameters. Third, the remaining weights of the parent networks are mutated by an adaptive, additive noise.

Once the offspring networks are generated, the networks are trained as described above and evaluated in order to select a new set of parents, forming the basis of the next cycle of evolution.

After finishing the evolutionary loop when either the required precision is achieved or a timeout occurs, the last generation of networks is fully trained and the best network output as well as the average outputs of all the survivors in the last generation are produced. The latter creates a committee of networks that might yield a lower error in case of independence of errors in a population that has not converged towards a single blueprint. Please see section $\mathrm{C}$ for a detailed description of the algorithm.

GETnet offers the following new, unique contributions to the field of temporal neural networks:

- Autonomous learning with minimal human supervision.

- General multidimensional temporal input-output format.

- General distributed memory.

- An adaptive mechanism to determine the structure, depth and distribution of short and long term memories.

- A novel, practical temporal minimum description length for regularization.

- An adaptive, noisy Lamarckian evolution for weight transfer. 
- Non-disruptive mutations for continuous phenotypical and structural change.

- Comprehensive framework integrating other useful established heuristics.

GETnet is also more flexible and comprehensive than the existing temporal neural network paradigms such as TDNN ${ }^{22}$, FIRnet $^{23}$, Elman $^{24}$, Jordan ${ }^{25}$, PRNN $^{26}$, and NARMA $^{27}$. In contrast to GETnet, all the mentioned networks need human experts to determine their memory and network structures as well as the other learning parameters (baby-sitting problem), which also entails the lack of an automated mechanism to determine the minimum required network size, an essential issue in generalization. Furthermore, none of the above paradigms offer an arbitrary distributed memory structure comprised of recurrent nodes and sub-circuits as well as delay lines of variable degrees. Please see the discussion at the end of section C "Conclusions and Future Work" for a more detailed explanation.

This document is divided into three main parts. Section A is this introduction. Section B goes through the relevant background theory. This section not only helps the reader to understand the fundamentals upon which GETnet is based, but also impresses upon the reader the sheer number of design parameters and issues that need to be determined in regular neural networks, leading to the "baby sitting" problem that GETnet avoids by automating almost everything. Section B is divided into four parts. The first part briefly describes some fundamentals of connectionist learning machines. The second and third parts go through linear and nonlinear neural networks, with each section being divided into static and dynamic networks. These three sections were mainly adopted from Principe's excellent new book ${ }^{48}$. The fourth and last part of section B describes evolutionary methods and their application in neural networks. Section $\mathrm{C}$ formally introduces the suggested General Evolutionary Temporal Neural Network or GETnet in detail, going through all the main modules. It is followed by the results and analysis of three simulations: 6 step prediction of Mackey-Glass chaotic series, 36 step prediction of Mackey-Glass chaotic series, and fingerprint perspiration sequence detection problem. A 
final discussion, conclusion, and future work section concludes section C. References and appendices go after this section and conclude this document.

Notation: In this document, bold letters (e.g. X) are used interchangeably for vectors or matrices. The arrow notation (e.g. $\vec{X}$ ) is used for vectors as well. Formula numbers begin with a letter that denotes their section, e.g. (B10), (C23), and so on. 


\section{B: BACKGROUND}

\section{B1 Classification Theory}

Any artificial or biological adaptive system in interaction with its environment needs to classify given inputs from the external world in order to produce the required response. The system has to preprocess its inputs, extract features, select a salient subset, and then make a sound decision by assigning input to a predefined class for supervised classification or cluster it into emerging classes in case of unsupervised classification. Here a very short survey of some fundamentals of supervised pattern recognition and its relation to artificial neural networks is presented. Artificial Neural Networks (or in short ANNs) can realize (optimal) adaptive statistical nonparametric classifiers in a fault tolerant, distributed presentation suitable for parallel hardware. ANNs can also implement unsupervised classifiers which will not be discussed here since this dissertation focuses on supervised learning.

The events from the external world can be expressed as a stream of Ddimensional vectors, with $\mathrm{D}$ being the number of basic acquisition elements (e.g. number of transducer cells). The elements of such vectors can be the pixel intensities from a two dimensional image, time samples of tactile transducers, etc. It is desired to reduce the high dimensional input into a lower salient subset so the input data appears in compact and disjoint clusters. These clusters are to be assigned to different classes according to the training data. The boundaries assigned by the classifier between input classes are called decision surfaces. Their choice has to minimize class assignment errors.

Linear regression networks are not suitable for classification since they try to minimize fitting error rather than classification error. Output nonlinearities called 
indicator functions are needed to bend regression hyper planes towards the class-specific numerical tags.

Optimal Bayesian Classifiers: These statistical classifiers are based on minimizing a misclassification risk given that the class conditional probabilities are known $^{28}$. Consider a vector $\mathbf{X}$ (random variable), and classes $c_{i}$ with given probability density or mass functions. The loss function $L\left(c_{i}, c_{j}\right)$ is the price paid when the classifier decides $\mathbf{X} \in \mathrm{c}_{\mathrm{i}}$ while in fact $\mathbf{X} \in \mathrm{c}_{\mathrm{j}}$. Using a posteriori probability $P\left(c_{i} \mid \boldsymbol{X}\right)$, the risk of a classifier for each pattern $\mathrm{c}_{\mathrm{i}} R\left(c_{i} \mid \boldsymbol{X}\right)$ is defined as the expected value of the loss $L\left(c_{i}, c_{j}\right)$ :

$$
R\left(c_{i} \mid \vec{X}\right)=\sum_{j} L\left(c_{i}, c_{j}\right) P\left(c_{j} \mid \vec{X}\right)
$$

Obviously for $\mathrm{i}=\mathrm{j} L\left(c_{i}, c_{j}\right)=0$. R should be minimized for an optimal classifier. A Bayesian classifier is optimal since for a given conditional probability it provides the best decision for minimizing the risk as defined in (B1). Using the above idea, if $L\left(c_{i}, c_{j}\right)=1$ for all $\mathrm{i} \neq \mathrm{j} 0$, one can obtain a simpler condition for classification

$$
\boldsymbol{X} \in c_{i} \text { if } P\left(\boldsymbol{X} \mid c_{i}\right) P\left(c_{i}\right)>P\left(\boldsymbol{X} \mid c_{j}\right) P\left(c_{j}\right) \quad \forall j \neq i
$$

For a simplified two class optimal classifier one can find a boundary $\mathbf{X}=\mathbf{T}$ such that

$$
p\left(\boldsymbol{X} \mid c_{1}\right) P\left(c_{1}\right)=p\left(\boldsymbol{X} \mid c_{2}\right) P\left(c_{2}\right)
$$

This is the optimal classifier's decision boundary, which depends on the classes' conditional distributions (e.g. means and variances for Gaussian distributions). Probability of overall classification error will be $\mathrm{P}\left(\mathbf{X}\right.$ classified $\in \mathrm{c}_{1}$ while $\mathbf{X}$ really $\left.\in \mathrm{c}_{2}\right)+\mathrm{P}\left(\mathbf{X}\right.$ classified $\in \mathrm{c}_{2}$ while $\mathbf{X}$ really $\in \mathrm{c}_{1}$. That is 


$$
P_{\text {error }}=\int_{\vec{X}>\vec{T}} p\left(\vec{X} \mid c_{1}\right) P\left(c_{1}\right) d \vec{X}+\int_{\vec{X}<\vec{T}} p\left(\vec{X} \mid c_{2}\right) P\left(c_{2}\right) d \vec{X}
$$

Generally speaking, the classification error is a function of both the class variances and means, thus the metric for classification (separability) should not merely be Euclidean, but it should also include class dispersion. An example of such a metric is Mahalanobis distance $^{29}$, which is proportional to $\frac{\|x-\mu\|}{\sigma}$, the distance of point $\mathbf{X}$ from a cluster with mean $\mu$ and standard deviation $\sigma$.

Discriminant Functions: The scaled likelihood $p\left(\boldsymbol{X} \mid c_{i}\right) P\left(c_{i}\right)$ or any monotonically increasing function of it such as the logarithmic function can constitute a discriminant function $\mathrm{g}_{\mathrm{i}}(\mathrm{x})$ so that if $\vec{X} \in c_{i}$ then it maximizes the corresponding discriminant function $\mathrm{g}_{\mathrm{i}}$ amongst other classes' discriminant functions like $\mathrm{g}_{\mathrm{j}}: g_{i}(\vec{X})>g_{j}(\vec{X}), \forall j \neq i$. Intersections of discriminant functions $\mathrm{g}_{\mathrm{i}}(\mathbf{X})$ are the decision surfaces, which partition input (or pattern) space into regions associated with each class.

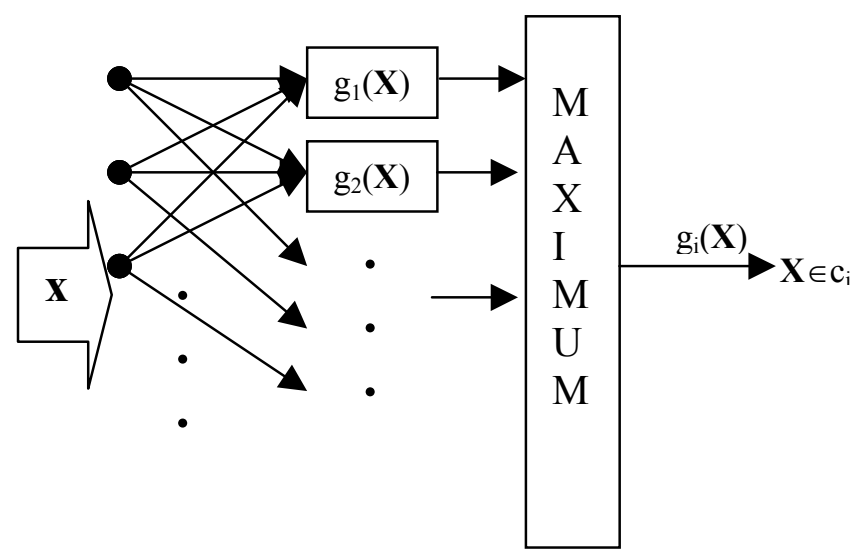

Figure 1 Classifier based on discriminant functions $g_{i}(X)$. 
Kernel-based Machines: These classifiers try to make given classes linearly separable by a nonlinear mapping from the input space to an intermediate space. Their behavior can be described by Cover's theorem ${ }^{30}$ which states that through nonlinear transformations, any classification task can become linearly separable in a sufficiently high dimensional intermediate space (i.e. the feature space). More specifically, assume $\mathrm{N}$ patterns $P=\left\{\vec{X}_{1}, \vec{X}_{2}, \ldots \vec{X}_{N}\right\}$ in the input space. $P$ can be categorized into two classes (a dichotomy) in $2^{\mathrm{N}}$ different ways, which can be considered as all the possible subsets of $P$ and their complements $\left\{p_{i} p_{i}^{c}\right\}, \forall p_{i} \subseteq P$.

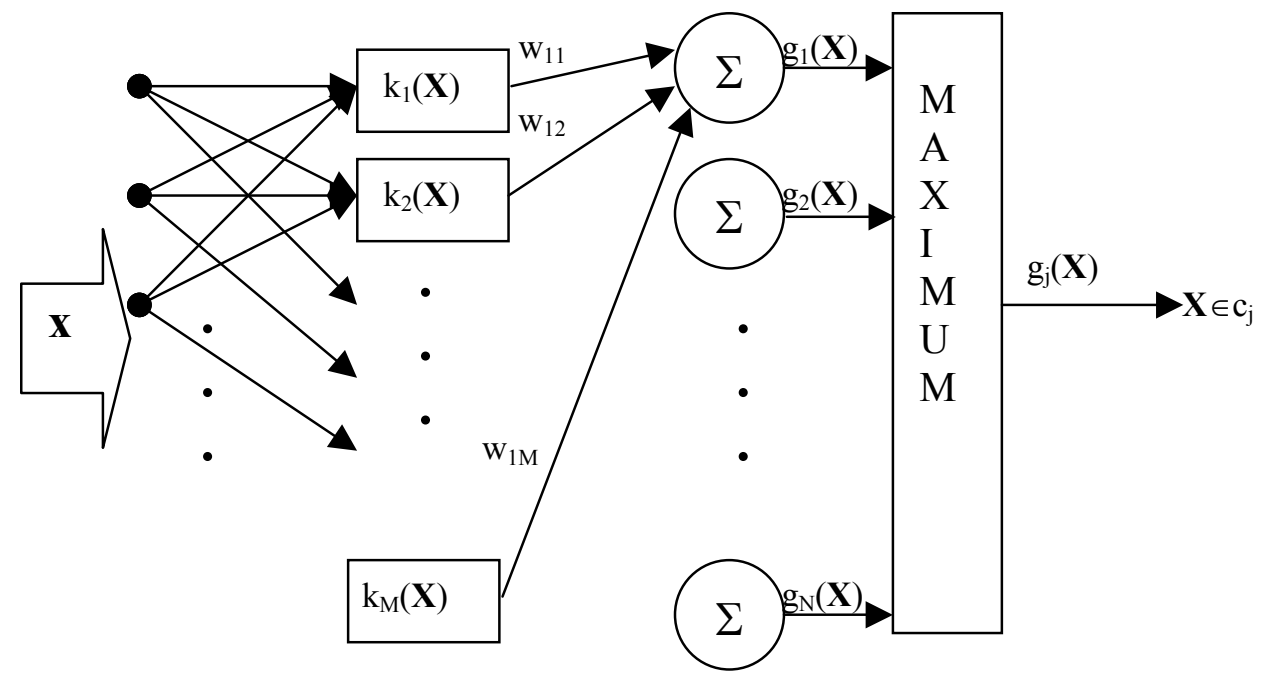

Figure 2 A kernel-based classifier.

$\mathrm{k}_{1}(\mathbf{X}), \mathrm{k}_{2}(\mathbf{X}), \ldots \mathrm{k}_{\mathrm{M}}(\mathbf{X})$ are the kernel functions in charge of nonlinear mapping of the input space into feature space and $\mathrm{g}_{1}(\mathbf{X}), \mathrm{g}_{2}(\mathbf{X}), \ldots . \mathrm{g}_{\mathrm{N}}(\mathbf{X})$ are the discriminants, where $g_{j}(\vec{X})=\sum_{i=0}^{M} w_{j i} k_{j}(\vec{X}), \quad w_{0}=b, k_{0}(\vec{X})=1$. The largest discriminant output indicates the classifier's decision. For instance, if kernels $\mathrm{k}_{\mathrm{i}}$ implements $x_{i}, x_{j}, x_{i}^{2}, x_{j}^{2}$, $x_{i} x_{j}, \ldots$ then $g_{i}(\boldsymbol{X})$ can implement a quadratic discriminant function obtained from the logarithm of Gaussian-distributed classes, and so forth. 
Cover showed that the probability of any such randomly chosen dichotomy being correctly classified by the above kernel-based machine is

$$
P_{N}(M)=\left\{\begin{array}{cc}
\left(\frac{1}{2}\right)^{N-1} \sum_{i=0}^{M}\left(\begin{array}{c}
N-1 \\
i
\end{array}\right) & M<N \\
1 & M \geq N
\end{array}\right.
$$

Where each of the $\mathrm{N}$ inputs is mapped nonlinearly to a $\mathrm{M}$-dimensional feature space and classified by $2^{\mathrm{N}}$ linear discriminants. (B5) shows that for $M \geq N$ i.e. feature space dimension equal or greater than number of input data points, this machine can always classify any dichotomy correctly. For $M<N$, the given probability function has a sharp knee at $N=2(M+1)$ where $P_{N}(M)$ starts to decrease rapidly. This best performance tradeoff neighborhood (i.e. the maximum number of entries in input space that can be classified with a small error into any dichotomy for a given machine) is defined as $\mathrm{C}$, the learning machines' capacity:

$$
C=2(M+1)
$$

For a linear classifier, one can assume $\mathrm{k}_{\mathrm{i}}=1$ (a direct connection for each input line to the output linear discriminants) and thus $\mathrm{M}=\mathrm{D}$ and $\mathrm{C}=2(\mathrm{D}+1)$.

Kernel-based machines de-couple machine capacity from input space dimension by going to a higher dimension feature space, where data clusters become more sparse and thus easier for linear separation. However, bigger classifiers need many more training points, which almost never are available. This leads to a famous paradoxical situation known as curse of dimensionality and peaking phenomena. The high dimensional problem should be more separable, but the higher number of free parameters, given the limited number of training samples, will degrade the performance (e.g. Trunk's example $\left.{ }^{31}\right)$. On the other hand, by reducing the number of features we decrease the input- 


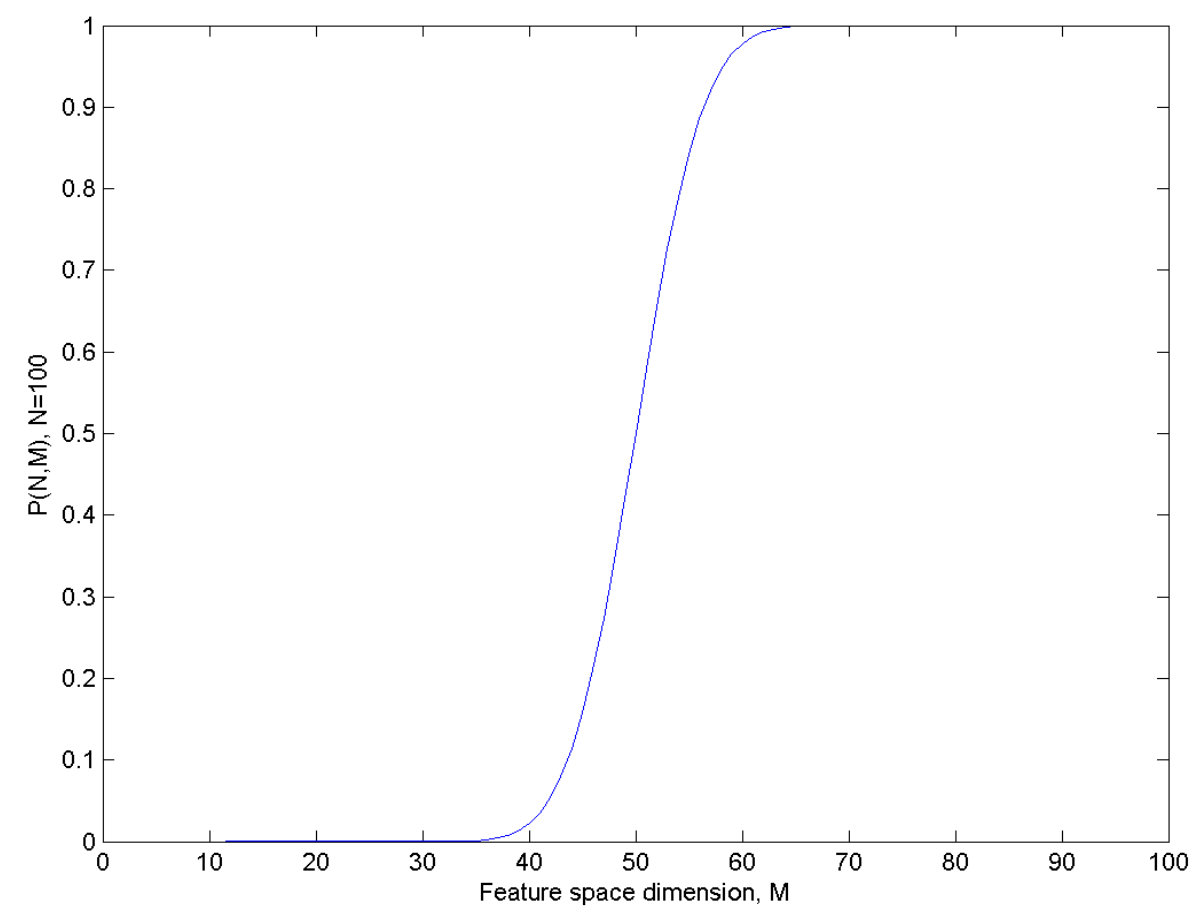

Figure 3 Plot of $\mathrm{P}_{\mathrm{N}}(\mathrm{M})$ demonstrates Cover's Theorem.

dimension and thus have fewer parameters to estimate, but at the same time reduce the separability given by Cover's theorem. The problem is that there are no exact rules describing the number of required salient feature and free parameters versus the size of the training set. This is one of the problems that will be addressed by the evolutionary design of the suggested evolutionary temporal neural networks, or GETnet (please see section C).

A related class of neural networks is the Support vector machine (SVM). SVM was introduced by Vapnik ${ }^{32,33}$ based on the concept of kernel machines where the input space is projected into a higher dimension kernel space. As mentioned above, the dimension of the kernel space can be made high enough so that the classes become linearly separable. SVM then chooses the largest margin discriminant using algorithms such as Adatron ${ }^{34}$ that find the projected data support vectors that are closest to the class margins and place the decision surface in between accordingly to achieve best generalization with the given training set. SVM can solve some of complex classification 
problems such as the intertwined spirals ${ }^{35}$ much better and faster. However, the kernel Adatron algorithm assigns one kernel per data point, which makes it expensive for large amounts of data. Furthermore, SVM's reliance on support vectors in feature spaces might make it sensitive to outliers, and most importantly SVM does not address temporal structures.

Neural Networks as Optimal Bayesian Classifiers: As expressed earlier, an optimal classifier with minimum error can be built based on a posteriori probability. That is, probability of an outcome given an observation. For a neural network, it translates into the probability of an output y given the input(s) $\mathbf{X}, P(y \mid \boldsymbol{X})$. It can be shown that under certain conditions, a neural network can realize an optimal Bayesian classifier by learning a posteriori probability of target values given the observed inputs. Artificial neural networks implement this scheme robustly in a distributed manner and learn nonparametrically from the examples.

The Bias-Variance Dilemma: consider a simple 1-D curve-fitting problem. One can exactly fit a polynomial of the degree $\mathrm{N}$ to $\mathrm{P}$ sample points provided that $\mathrm{N} \geq \mathrm{P}-1$. However, if the degree of the polynomial is less than P-1, the regression generally cannot accommodate all the sample points (over-constrained case) and thus the model will have bias. On the other hand, if the regression has more or even just enough parameters to fit the samples, it might overshoot or undershoot for the points in between compared to the actual test data (under-constrained case). In this case our model is suffering from variance (figure 4).

In general, one wishes to approximate the actual phenomena (function) $f$ in $d=f(\vec{X})$ by an adaptive approximant $y=\hat{f}(\vec{X}, \vec{W})$ so that $y$ follows $d$ as closely as possible. Thus for function approximation one needs to find an approximant that provides the minimum model variance and bias at the same time by choosing the right number of free parameters or model complexity. The complexity is also proportional to the number of elementary functions, kernels, layers, etc. A large number of free parameters enables- 


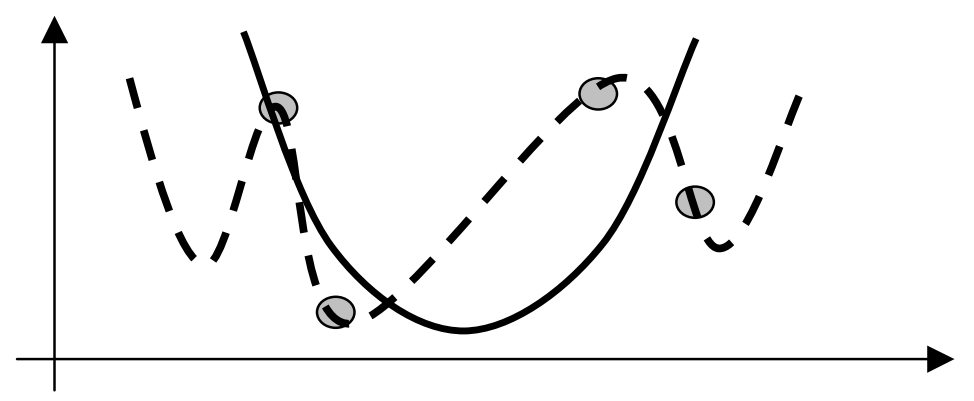

Figure 4 Solid curve shows fitting a quadratic to 4 points (not enough degrees of freedom, model bias). Dashed curve shows fitting a $6^{\text {th }}$ order curve (extra degrees of freedom, model variance).

the model to memorize the training pattern but this usually hurts generalization by introducing variance in the regions not covered by the training set (don't-care areas in training). Reducing the number of parameters reduces unwanted variance as well, but at the cost of over-simplifying the network and introducing an inescapable bias error. This trade-off in choosing the right model complexity is called the bias-variance dilemma. Note that the average of different models in a committee of classifiers tends to cancel out the variance. Early stopping in cross-validation tries to stop an under-constrained model from introducing extra variance. This problem is being addressed by evolutionary design of GETnet (see the following and section C).

Regularization: in order to include the above-mentioned phenomena in the design of learning machines, instead of minimizing just the training error one can minimize a new criterion that includes system complexity as well. This way a better design that can minimize both the training error and model variance can be achieved. One such cost function is the Akaike information criterion (AIC) which includes a linear penalty for system size

$$
A I C(M)=\ln (J)+\frac{2 M}{N}
$$


$M$ is the number of model's free parameters (complexity) and $N$ is the size of the training set. Larger training sets require more parameters to encompass their possibly more complicated mapping. This is accommodated by inclusion of $N$. This way one can use more (or even all) of available data for training since limiting the number of parameters reduces the unwanted model variance for the unseen data which is also the purpose of cross-validation. Note that counting just the number of parameters is not a good measure for multilayer neural networks since the role of each layer is very different from that of say a single layer, kernel based machine. This is one the reasons behind the new timebased regularization system of GETnet.

More generally, the extra penalties added to the original cost function are called regulizers $\Gamma$

$$
J_{\text {new }}=J+\lambda \Gamma
$$

where $J$ is the original error (e.g. MSE), $\lambda$ is the regularization constant, and $\Gamma$ is the regulizer. $\Gamma$ can penalize different aspects of the learning machine, including the size of the first and second derivatives of the output vs. the inputs in order to keep the model variance down.. Interestingly, a class of kernel-based machines called Radial Basis Function Neural Networks can be derived as a solution for Tikhonov regulization expressed in $(\mathrm{B} 9)^{36}$. In section $\mathrm{C}$, a more practical regularization method is introduced for use in GETnet which is based on the minimum length of the neural network description on the hosting machine and the actual execution times. 


\section{B3 Artificial Neural Networks}

Artificial Neural Network (ANN) is a connectionist model motivated by biological neural networks. It generally consist of simplified neuron-like nodes interconnected through a set of adaptable weights. ANNs derive many of their characteristics from their biological counterparts, including massively parallel connections for fault tolerant parallel processing, local computation, decentralized control, as well as associative and distributed memories. Using the hierarchy of minds, brains, and machines used in the study of brain systems, ANNs fall under the machines category. That is, the engineering aspect of these connectionist models that are applicable to real world problems are of the most interest. It should be emphasized that the aim of this research is not modeling the biological neural networks, but rather using general ideas from their structure and function to help making better intelligent machines. However, while the field of artificial neural networks and computational intelligence in general is continuously utilizing the ideas taken from biological systems, ANNs are also used by medical researchers to explain the mechanisms of biological brain systems $^{37,38,39,40}$.

To design an adaptive system in general and a neural network in particular, be it linear or nonlinear, one has to choose system's topology (including component models), a performance criterion, and a learning algorithm. Training data collected for such a system should be sufficient in number, capture fundamental principles at work, and have the least observation noise. Such a system can be used for several purposes, including system identification (finding input-output relations while treating the studied system as a black box) and classification, among the other things. Among these three criteria, the first has been the most complicated to answer. GETnet provides an automated solution to this problem (please see section C). 


\section{Topology}

Topology plays a very important role in the system performance. As a connectionist system, incorporation of appropriate nodes as well as their number and interconnections directly dictates the computational and adaptive capabilities of an ANN. Topology and network architecture also heavily influence the bias-variation dilemma and generalization capability of a network.

\section{Performance Measures}

As stated earlier, a learning system needs a performance criterion to determine how good its output is. One popular measure for supervised learning is the mean of squared errors, or MSE

$$
J=\frac{1}{2 N} \sum_{i=1}^{N} \varepsilon_{i}^{2}
$$

This criterion also has special significance in probabilistic interpretation of learning, since a neural network with MSE performance criterion can implement Baysian optimal classifiers.

To minimize the MSE, one can set the partial derivatives of this error function to zero with respect to the adaptive parameters. This is especially true for linear neural networks since MSE creates a non-negative parabolic error surface with respect to the parameters of such networks. For nonlinear systems, iterative algorithms such as gradient descent are used.

MSE belongs to a more general family of norms called $\mathrm{L}_{\mathrm{P}}$, which is the output error to power P. Performance measures can include more than the output error, including 
penalty terms for topology as described in regularization. Temporal ANNs can use similar performance measures that are summed over the duration of interest. Even though ANNs usually use simplified single-objective performance criteria, multi-objective performance criteria in general are also receiving attention recently ${ }^{41}$.

The following visualization tools are also useful for describing the learning and testing phases of neural networks:

Performance Plots: also known as the learning curve or MSE plots include graphing of MSE vs. iteration number. One can also plot weight tracks (i.e. plot each connection weight vs. iteration number) for more insight. Weight tracks may demonstrate over-damped, critically damped, or divergent behaviors based on the value of adaptation step size $\eta$, with small step sizes resulting in a sluggish over-damped convergence and large steps making the learning more prone to unstable and divergent regimes.

False Accepts and False Rejects, and the Confusion Matrix: a simple but effective way to visualize and compare classifying machines is through the creation of a confusion matrix using test data results. The matrix for a dichotomy follows. This method can also be applied if more than two classes are involved. Having a diagonal matrix will be the best case (no misidentification). Since this matrix is supposed to be built using the test data set which is not used during the training, a populated diagonal also implies good generalization. Each off-diagonal element indicates a class that was identified as another. Furthermore, one can see which classes are more separable. Thus this will provide the experimenter with valuable performance information that is not evident in other measures such as MSE and weight tracks. 
Table 1 Confusion matrix.

\begin{tabular}{|c|c|c|c|}
\hline Actual & Class 1 & Class 2 & Total Actual \\
\hline Class 1 & $\begin{array}{c}\mathrm{C}_{11} \\
\text { Correct }\end{array}$ & $\begin{array}{c}\mathrm{C}_{12} \\
\text { Misidentify }\end{array}$ & $\mathrm{C}_{11}+\mathrm{C}_{12}$ \\
\hline Class 2 & $\begin{array}{c}\mathrm{C}_{21} \\
\text { Misidentify }\end{array}$ & $\begin{array}{c}\mathrm{C}_{22} \\
\text { Correct }\end{array}$ & $\mathrm{C}_{21}+\mathrm{C}_{22}$ \\
\hline $\begin{array}{c}\text { Total Neural } \\
\text { Network }\end{array}$ & $\mathrm{C}_{11}+\mathrm{C}_{21}$ & $\mathrm{C}_{12}+\mathrm{C}_{22}$ & $\begin{array}{c}\text { Total } \\
\text { Samples }\end{array}$ \\
\hline
\end{tabular}

\section{Learning Algorithms}

A learning algorithm is the search method that changes the system's free parameters such that the performance measure is optimized. For supervised learning, besides an optimality criterion and learning method, one needs desired input-response pairs. One method used extensively in first-order supervised adaptation algorithms for many types of neural network is gradient descent on the error function. In conjunction with the chain rule for multivariate functions, gradient descent is the cornerstone of the famous and powerful Least Mean Squares (LMS) family of algorithms. LMS is local in two different senses. First, because the nodes in a neural network can take part in the global (network-wide) calculation for optimal performance just by using the local signals from immediate nodes. Second, LMS finds local error minima and by itself cannot distinguish between local and global answers. Enhancements such as adding momentum and noise during the training phase or use of global search methods such as evolutionary techniques can help alleviate this problem, as described during the later sections. Other 
learning algorithm issues include choice of initial conditions and finding criteria to determine when the training should be stopped. 


\section{B3-1 Static Linear Neural Networks}

A learning linear system tries to adapt its parameters so that it can fit a hyperplane with minimal or no error to given data points. This is also known as linear regression. A neural network implementation of the linear regressor is called Adaline, which stands for Adaptive linear element.

The Adaline (linear regression) model explains the relationship $f$ in $d_{i}=f\left(\vec{X}_{i}\right)$ by minimizing the MSE. The first note of caution in using linear neural networks is the limitation imposed by the first order regression: a linear network cannot map the given data points $\left\{\left(x_{i}, d_{i}\right)\right\}$ well if they are not linearly correlated. One way to find out about the co-trends between given data is calculating the correlation coefficient. The correlation coefficient between $\mathrm{x}$ and $\mathrm{y}$ is defined as:

$$
r=\frac{\frac{\sum_{i}\left(x_{i}-\bar{x}\right)\left(y_{i}-\bar{y}\right)}{N}}{\sqrt{\frac{\sum_{i}\left(x_{i}-\bar{x}\right)^{2}}{N} \sqrt{\frac{\sum_{i}\left(y_{i}-\bar{y}\right)^{2}}{N}}}}=\frac{\operatorname{Cov}(x, y)}{\sigma(x) \sigma(y)} \quad-1 \leq r \leq+1
$$

$\mathrm{r}=+1$ shows perfectly positive linear correlation between $\mathrm{x}$ and $\mathrm{y}, \mathrm{r}=-1$ shows perfectly negative linear correlation between them, and $\mathrm{r}=0$ means $\mathrm{x}$ and $\mathrm{y}$ are uncorrelated. The closer the coefficient to \pm 1 , the better a linear fit. Thus if the training data covers most of possible cases with a correlation coefficient close \pm 1 , then we can use a linear regression model for prediction of unseen data (generalization). This coefficient can also be used to show quality of prediction in any neural network model by setting $x_{i}$ to the actual target values and $y_{i}$ to the corresponding prediction, as shown in the results section for Mackey-Glass chaotic series prediction tasks. 


\section{Neuron Model}

The model used in linear neural networks is simply a weighted average of the inputs, similar to that of the linear regression $y=b+w_{1} x_{1}+w_{2} x_{2}+\ldots+w_{D} x_{D}$. However, the adaptation and implementation approaches are different.

\section{Training Algorithms}

Linear neural networks can utilize different algorithms to change their weights in order to minimize their error. As in the linear regression case, if the number of free parameters is equal or more than the number of training data a perfect linear fit can be achieved (under-constrained). In this case the training data is memorized, which usually is not the best case for fitting the test data (poor generalization). If the system has fewer free parameters i.e. weights, (over-constrained), one can use an iterative algorithm such as LMS to find the minimum-error fit as described below.

\section{First Order Algorithms: LMS Method}

Generally speaking, for a given dataset of $\mathrm{N}$ input-target pairs $\left\{\left(\mathbf{X}_{\mathrm{i}}, \mathrm{d}_{\mathrm{i}}\right)\right\}$, $\mathrm{i}=1,2, \ldots, \mathrm{N}$ and $\mathbf{X}_{\mathrm{i}}=\left(\mathrm{x}_{\mathrm{i} 1}, \mathrm{x}_{\mathrm{i}} 2, \ldots \mathrm{x}_{\mathrm{iD}}\right)$, it is desired to fit a D-dimensional hyper plane In vector (matrix) notation:

$$
\widetilde{d}_{i}=y_{i}=\sum_{j=0}^{D} w_{j} x_{i j}=\vec{W} \cdot \overrightarrow{X_{i}}=W^{T} \cdot X_{i}, \quad \vec{W}=\left[\begin{array}{c}
w_{0} \\
w_{1} \\
\vdots \\
w_{D}
\end{array}\right], \vec{X}=\left[\begin{array}{c}
x_{0} \\
x_{1} \\
\vdots \\
x_{D}
\end{array}\right] ; w_{0}=\text { bias, } x_{0}=1
$$


One can find the optimal weight set $\overrightarrow{W^{*}}$ to minimize $M S E=J(\vec{W})=\frac{1}{2 N} \sum_{i=1}^{N} \varepsilon_{i}^{2}, \quad \varepsilon_{i}=d_{i}-\tilde{d}_{i}$ by setting $\frac{\partial J}{\partial w_{k}}=0, k=0,1, \ldots D$ and solving the resulting $\mathrm{D}+1$ equations. For iterative solution which is preferred for adaptive systems, one can use the gradient-descent LMS algorithm. Both methods are described below.

Analytical Solution: The input autocorrelation matrix R (from D+1 input lines) is defined as

$$
R=\frac{1}{N} \sum_{i=1}^{N} R_{i}, \quad \text { where } R_{i(D+1 \times D+1)}=X_{i} X_{i}^{T}
$$

Since $r_{m n}=$ mean $\left(x_{m} x_{n}\right)=$ mean $\left(x_{n} x_{m}\right)$, then $r_{m n}=r_{n m}$ and $R$ is symmetric.

The input-output cross-correlation matrix $\mathrm{P}$ is defined as

$$
P=\frac{1}{N} \sum_{i=1}^{N} P_{i}, \quad \text { where } P_{i(D+1 \times 1)}=d_{i} X_{i}
$$

Since one can write $\vec{\nabla}_{W}()=.\sum_{i} \hat{u}_{i} \partial / \partial w_{i}($.$) , so grad (.) is a linear operator with$ derivative-like properties. Thus one can write

$$
\begin{gathered}
J(\vec{W})=\frac{1}{2 N} \sum_{i=1}^{N}\left(d_{i}-\tilde{d}_{i}\right)^{2}, \quad \tilde{d}_{i}=W^{T} X_{i}=X_{i}^{T} W \\
\vec{\nabla}_{\vec{W}} J=\frac{1}{2 N} \sum_{i=1}^{N} 2\left(d_{i}-\widetilde{d}_{i}\right)\left(0-\vec{\nabla} \widetilde{d}_{i}\right)=-\frac{1}{N} \sum_{i=1}^{N}\left(d_{i}-X_{i}^{T} W\right)\left(X_{i}\right) \\
=-\frac{1}{N} \sum_{i=1}^{N} X_{i} d_{i}+\frac{1}{N} \sum_{i=1}^{N} X_{i} X_{i}^{T} W=-\frac{1}{N} \sum_{i=1}^{N} d_{i} X_{i}+\left(\frac{1}{N} \sum_{i=1}^{N} X_{i} X_{i}^{T}\right) W=-P+R W
\end{gathered}
$$




$$
\begin{gathered}
\vec{\nabla}_{\vec{W}} J=R W-P ; \quad \text { For } J_{\text {min }}: \vec{\nabla}_{\vec{W}} J=\overrightarrow{0} \rightarrow P=R W^{*}: \\
W^{*}=R^{-1} P \quad \text { (B15) }
\end{gathered}
$$

Iterative Solution: instead of computing the optimal $\mathbf{W}^{*}$ from (B15) for minimum error, one can do an iterative search over the error surface. Since $\vec{\nabla} J(\vec{W})$ points towards the maximum (rate of change) direction, then $-\vec{\nabla} J(\vec{W})$ points towards the minimum (fastest descending) direction of the error surface. To find the MSE gradient, one can write

$$
\begin{gathered}
\vec{\nabla}_{\vec{W}} J=\vec{\nabla}_{\vec{W}}\left(\frac{1}{2 N} \sum_{i=1}^{N} \varepsilon_{i}^{2}\right)=\frac{1}{2 N} \sum_{i=1}^{N} 2\left(d_{i}-\widetilde{d}_{i}\right)\left(0-\vec{\nabla} \widetilde{d}_{i}\right) \\
=-\frac{1}{N} \sum_{i=1}^{N}\left(\varepsilon_{i}\right)\left(\vec{X}_{i}\right)
\end{gathered}
$$

for single data point $\mathrm{i}=\mathrm{k}$ :

$$
\vec{\nabla}_{\vec{W}} J_{k}=\vec{\nabla}_{\vec{W}}\left(\frac{1}{2} \varepsilon_{k}^{2}\right)=\left(d_{k}-\widetilde{d}_{k}\right)\left(0-\vec{\nabla} \widetilde{d}_{k}\right)=-\varepsilon_{k} \vec{X}_{k}
$$

To move in the direction of steepest descent by a single sample gradient (say $\mathrm{k}^{\text {th }}$ ), one can write:

$$
\vec{W}_{k+1}=\vec{W}_{k}-\eta \vec{\nabla} J_{k}=\vec{W}_{k}+\eta \varepsilon_{k} \vec{X}_{k}
$$

$\eta$ is a small, positive step size which is also called learning rate. This is a noisy estimate since it is based on a single sample $\left(\mathrm{x}_{\mathrm{k}}, \mathrm{d}_{\mathrm{k}}\right)$ of the whole set of $\mathrm{N}$ points. This noise might 
be averaged out over many iterations. Iteration over the entire $\mathrm{N}$ data points is called an epoch.

Step Size Control: based on the above calculations one can show that

$$
W_{k+1}-W^{*}=(I-\eta R)^{k}\left(W_{0}-W^{*}\right)
$$

For convergence, it is sufficient that $\lim (I-\eta \Lambda)^{k} \stackrel{k \rightarrow \infty}{\longrightarrow} 0$, where

$$
\begin{aligned}
& \Lambda=\left[\begin{array}{cccc}
\lambda_{0} & 0 & \cdots & 0 \\
0 & \lambda_{1} & & \vdots \\
\vdots & & \ddots & 0 \\
0 & \cdots & 0 & \lambda_{D}
\end{array}\right] \text { and } \lambda_{0}, \lambda_{0}, \ldots, \lambda_{\mathrm{D}} \text { are the eigenvalues of } \mathrm{R} \text {. Then we should have } \\
& \left|1-\eta \lambda_{i}\right|<1 \text { which means } 0<\eta<\frac{2}{\lambda_{i}}, i=0,1, \ldots D \text {. So for converging step size } \\
& \eta<\frac{2}{\lambda_{\max }}
\end{aligned}
$$

If one considers step $\mathrm{k}$ as a discrete time, then the convergence time constant in the $\mathrm{i}^{\text {th }}$ direction $\left(\mathrm{w}_{\mathrm{i}}\right)$ will be $\tau_{i}=\frac{1}{\eta \lambda_{i}}$, implying a faster initial pace along the direction of largest eigenvectors (larger $\lambda$, smaller $\tau$ ), and continuing along smaller eigenvectors afterwards.

In order to achieve both speed and precision especially for nonlinear multilayer networks where the optimum step size cannot be calculated, one can use step size scheduling by starting with a larger step size for initial speed and then reduce it for accuracy near optimal weights (called learning-rate scheduling). The reduction of $\eta$ can be performed by using linear, geometric, or logarithmic schemes. This technique is also known as annealing. There are other general heuristics for the LMS adaptation that are described in the literature ${ }^{42}$. The above-mentioned details are just a small portion of all 
the intricacies that one should go through in order to design and implement even a simple neural network, a problem that GETnet tries to circumvent.

We must also mention two important modalities in training of neural networks: batch and online learning. Updating $\mathbf{W}_{\mathrm{i}}$ for each step is called online learning. One can use the same starting $\mathbf{W}$ for calculating all the $\Delta \mathbf{W}_{\mathrm{i}}$ in an epoch and then average them to get the new W. This is called batch learning. It involves fewer calculations and might provide a smoother convergence. Batch learning is also important in temporal neural networks, where each training pair represents a different moment in time. Such temporal batch training is called trajectory learning.

\section{Second Order Algorithm: Newton's Method}

Since we had $\vec{\nabla}_{\vec{W}} J=R W-P$, then $R^{-1} P=R^{-1}(R W-\vec{\nabla} J)$ or $W^{*}=W-R^{-1} \vec{\nabla} J$. Iteratively, one can write

$$
\vec{W}_{k+1}=\vec{W}_{k}-R^{-1} \vec{\nabla} J_{k}
$$

This modified gradient-based training method is also called the Newton's method. This method changes the direction of search for skewed error surfaces by $\mathrm{R}^{-1}$. The original gradient descent algorithm moves perpendicular to constant-error contours on the error

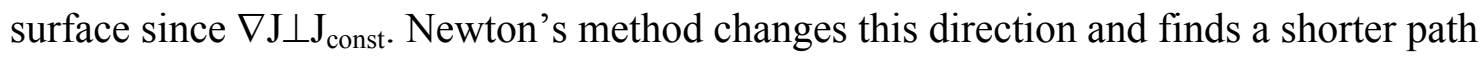
to $\mathrm{J}_{\min }$, because for skewed error surfaces contour plots from $\mathrm{J}=$ constant are non-circular and this method compensates for different time constants $\tau_{\mathrm{i}}$ in different directions. As one can see from (B21), this method can get stuck at saddle points where the gradient is zero. GETnet avoids this problem by adding adaptive noise components to the network weights, as described later in section $\mathrm{C}$. 
Modified Newton (LMS/Newton) algorithm: one can add a step size $\eta$ to the second term in (B21) and replace the gradient with the sample-based approximation from (B17) to get the iterative LMS/Newton form

$$
\vec{W}_{k+1}=\vec{W}_{k}+\eta R^{-1} \varepsilon_{k} \vec{X}_{k}
$$

\section{Lateral Inhibition}

The proposed network in section $\mathrm{C}$ can produce an arbitrary network structure, including those with lateral inhibition. The decorrelating capabilities of such a formation can shed a light into many of the capabilities of GETnet and will be briefly discussed here.

Consider the paths in figure 5 for the network signals $\mathrm{x}_{2}$ and $\mathrm{y}_{1}$

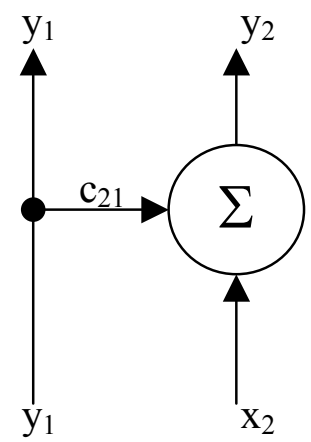

Figure 5 Simple lateral inhibition.

This is a simple lateral inhibition where $\mathrm{y}_{1}$ adds a negative lateral signal $\mathrm{c}_{21} \mathrm{y}_{1}$ to $\mathrm{x}_{2}$ so that

$$
y_{2}=x_{2}+c_{21} y_{1}
$$

The sample-based cross-correlation between $\mathrm{y}_{1}$ and $\mathrm{y}_{2}$ can be written as 


$$
r_{y_{1}, y_{2}}=\frac{1}{N} \sum_{n=1}^{N} y_{1}(n) y_{2}(n)=\frac{1}{N} \sum_{n=1}^{N} y_{1}(n)\left(x_{2}(n)+c_{21} y_{1}(n)\right)=\frac{1}{N} \sum_{n=1}^{N} y_{1}(n) x_{2}(n)+\frac{1}{N} \sum_{n=1}^{N} c_{21} y_{1}{ }^{2}(n)
$$

One then can easily choose a $c_{21}$ to decorrelate $\mathrm{y}_{1}$ and $\mathrm{y}_{2}$

$$
\frac{1}{N} \sum_{n=1}^{N} y_{1}(n) x_{2}(n)+\frac{1}{N} \sum_{n=1}^{N} c_{21} y_{1}^{2}(n)=0 \rightarrow c_{21}=-\frac{\sum_{n=1}^{N} y_{1}(n) x_{2}(n)}{\sum_{n=1}^{N} y_{1}^{2}(n)}
$$

so the strength of such decorrelating lateral inhibition is equal to (minus) the inputs' cross-correlation over the first signal's energy.

\section{LMS and Hebbian Learning}

$$
\begin{aligned}
& \text { According to (B18) } \vec{W}(n+1)=\vec{W}(n)-\eta \vec{\nabla} J(n)=\vec{W}(n)+\eta \varepsilon(n) \vec{X}(n) \text {, or } \\
& \qquad \vec{W}(n+1)=\vec{W}(n)+\eta d(n) \vec{X}(n)-\eta y(n) \vec{X}(n) \quad \text { (B26) }
\end{aligned}
$$

That is, the LMS algorithm for a linear node is composed of a forced-Hebbian term $\eta d(n) \vec{X}(n)$ that drives the weight vector towards the correlation of input-target values and an anti-Hebbian term $-\eta y(n) \vec{X}(n)$ that is depositing a decorrelation of input-output in the weight vector and driving the output towards zero, thus acting similar to the stabilizing term in Oja's rule ${ }^{43}$.

There is biological evidence for Hebbian learning, whereas LMS and backpropagation type of learning have not been clearly observed in biological nervous 
systems. However, it was shown above that Hebbian learning is a component of the LMS gradient descent learning. Moreover, there is emerging new evidence of gradient descent backpropagation learning in biological systems such as stem cell regulation ${ }^{44}$ as further indication of biological relevance of gradient descent-based learning paradigms.

To conclude this section for static linear neural networks, it must be mentioned that the reason for not introducing multi-layer linear ANN is the fact that combination of any $\mathrm{N}$ hidden layers of linear PEs will yield a linear transfer function, so such configuration is redundant and will degenerate to a single layer Adaline. 


\section{B3-2 Dynamic Linear Neural Networks}

Consider a delay line with D taps and D-1 delay elements receiving a timesampled signal $\mathrm{x}(\mathrm{n})$. As long as the sampling frequency for $\mathrm{x}(\mathrm{n})$ is at least twice the highest frequency of interest in $x(t), x(n)$ will represent the input signal $x(t)$ faithfully

(Nyquist's theorem ${ }^{45}$ ). The delay line can be considered as a short-term memory (STM) since the system will remember (D-1)* $\mathrm{T}_{\text {sampling }}$ of the input signal's history. Three different neuron models, namely moving average (MA), autoregressive (AR), and autoregressive-moving average (ARMA $)^{46}$ are used for temporal linear ANNs. The first two can be considered as special cases of ARMA.

Moving Average Model: a D-point weighed average of the input from a tapped input delay line represents a Moving Average (MA) filtering of x(n):

$$
y(n)=\sum_{i=0}^{D-1} w_{i} x(n-i)
$$

Since the impulse response of (B27) exists only for D clock ticks, it is also referred to as a Finite Impulse Response or FIR filter. This form is easily realized from the (zero bias) linear model studied earlier, with the input vector defined by the instantaneous contents of the delay line:

$$
\vec{X}(n)=\left[\begin{array}{c}
x(n) \\
x(n-1) \\
\vdots \\
x(n-D+1)
\end{array}\right]
$$

Similarly the discrete-time desired output is denoted by $d(n)$ and the resulting error is 


$$
\begin{aligned}
& \varepsilon(n)=d(n)-y(n) \\
J= & \frac{1}{N} \sum_{n=1}^{N} \varepsilon^{2}(n)
\end{aligned}
$$

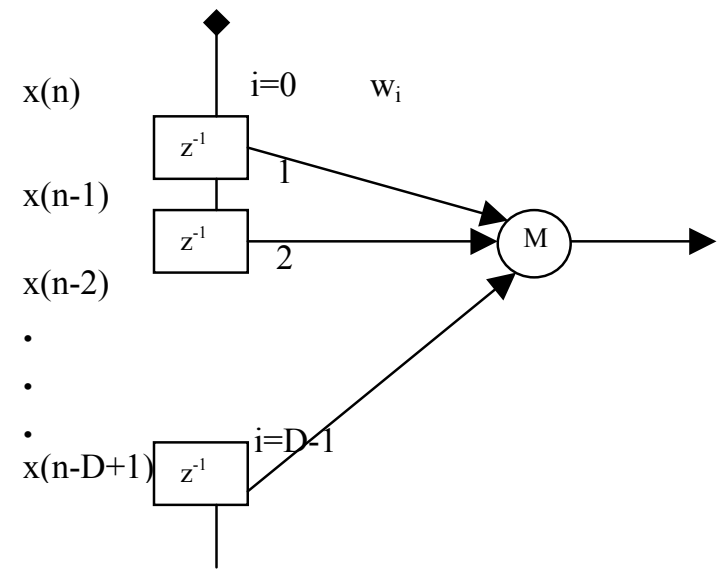

Figure 6 A moving-average linear neuron.

We also can extend this temporal interpretation to auto-correlation and crosscorrelation matrices $\mathrm{P}$ and $\mathrm{R}$ :

$$
P=\frac{1}{N} \sum_{n=1}^{N} P(n), \text { where } P(n)_{(D \times 1)}=d(n) X(n)
$$

and

$$
R=\frac{1}{N} \sum_{n=1}^{N} R(n), \quad \text { where } R(n)_{(D \times D)}=X(n) X^{T}(n)
$$

where $\mathrm{N}$ is the number (length) of time samples available and $\mathbf{X}_{\mathrm{Dx} 1}(\mathrm{n})$ is the timesampled input signal $x$ in the delay line as shown in figure 6. If the input-target samples are ergodic, the above time averages can be replaced by the ensemble averages (or 
statistical expected values). It can be seen that for temporal interpretation one can just replace the sample index $i$ with the discrete time index $n$ and add an input tapped delay line to a linear neuron according to Figure 6 for constructing $\mathbf{X}(\mathrm{n})$, and thus all the previously derived results still hold. The time series can be padded with zeros for unavailable samples (e.g. negative indices). There are other algorithms such as RLS (Recursive Least Squares) for finding the optimal weights for the linear node in (B27) and minimize the error in (B29). The linear MA filter of (B27) is also called a Wiener filter.

Besides the usual applications of linear regression, one can train this linear neuron for $\mathrm{d}(\mathrm{n})=\mathrm{x}(\mathrm{n}+\mathrm{k})$ to do prediction, with $\mathrm{k}$ usually set to 1 . In this case since only the input is being used for training, so it can be considered as some type of unsupervised learning. This mode of operation is used to test GETnet with Mackey-Glass chaotic time series (please see section C). Other applications of temporal linear neural networks include interference and echo-cancellation, line enhancement and adaptive control, to name a few.

Auto Regressive Model: this node model comes with a recursive time-delayed connection to combine its past outputs with its present input

$$
y(n)=a_{0} x(n)+\sum_{i=1}^{D} w_{i} y(n-i)
$$

Here the tapped delay line is implemented at the output of the linear node and fed back to the input. This constitutes the auto-regressive (AR) model.

Auto Regressive Moving Average Model: one can combine the moving average model of (B27) with the auto-regressive model of (B32) to get a more flexible model (and at the same time computationally more expensive to train) called ARMA:

$$
y(n)=\sum_{i=0}^{Z} a_{i} x(n-i)+\sum_{j=1}^{P} b_{j} y(n-j)
$$


Because of the recursive connections from the output, the impulse response of the linear neurons of (B32) and (B32) are stretched infinitely in time, so they are also called Infinite Impulse Response or IIR filters as well. This makes AR and ARMA models prone to becoming unstable, whereas the MA model will always have a bounded, finite response, provided that it is given a bounded, finite input (bounded in, bounded out or BIBO stability).

The frequency (steady state) responses of the MA, AR, and ARMA models can be inspected from their transfer functions in z-domain:

$$
\begin{gathered}
H_{M A}(z)=\frac{Y(z)}{X(z)}=\sum_{i=0}^{D} w_{i} z^{-i} \\
H_{A R}(z)=\frac{Y(z)}{X(z)}=\frac{a_{0}}{1-\sum_{i=1}^{D} w_{i} z^{-i}} \\
H_{A R M A}(z)=\frac{Y(z)}{X(z)}=\frac{\sum_{i=0}^{Z} a_{i} z^{-i}}{1-\sum_{j=1}^{P} b_{j} z^{-j}}
\end{gathered}
$$

It can be seen that the MA model only has zeros and AR is an all pole model. ARMA has both poles and zeros and thus the most versatile. In addition, because of their poles, AR and ARMA can oscillate and become unstable. All these models can be realized by a general linear neuron with summing tap delays both on its input and output paths, realizing different variations of the difference equation

$$
\sum_{i=0}^{Z} a_{i} x(n-i)=\sum_{j=0}^{P} b_{j} y(n-j)
$$


Other Memory Kernels: besides the simple delay line, one can use more complex memory structures (also called memory kernels) such as memories with recurrent connections in different configurations like a tapped line. These recurrent memory kernels such as Gamma memory units will be explained in nonlinear dynamic neural networks, section B3-4. 


\section{B3-3 Static Nonlinear Neural Networks}

Nonlinear neural networks are interconnected networks of adaptive, nonlinear elements. They are capable of creating arbitrary discriminant functions, including that of an optimal classifier. Nonlinear ANNs are usually arranged in different layers and can be trained with different algorithms, including the popular backpropagation algorithm that is based on gradient descent LMS technique and is applicable to supervised adaptation of multi-layer ANNs with differentiable nonlinearities.

\section{Neuron Model}

The popular neuronal model used in these ANNs is a linear neuron cascaded with a saturating nonlinearity $f$. The hyperplane created by the linear weighted summation creates the decision surface

$$
n e t=W^{T} X ; x_{0}=1, w_{0}=b ; \quad y=f(\text { net })
$$

For decision surface net $=W^{T} X=0$, i.e. the weight vector $\mathbf{W}$ is normal to the decision surface. For instance For $\mathrm{D}=2$ we have $n e t=w_{1} x_{1}+w_{2} x_{2}+b=0$ or $x_{2}=-\frac{w_{1}}{w_{2}} x_{1}-\frac{b}{w_{2}}$, which is a line determined by the weight ratios. Even though the placement of the decision surface does not change as long as the ratios remain the same, the transition band through the nonlinearity bending of the hyperplane does. This is because larger $w_{i}$ s create a steeper hyperplane that bends faster and thus creates a narrower transition band (see figure 7). Introduction of the nonlinear activation function $f$ may introduce multiple local minima and saddle points in the error function $J=\frac{1}{2 N} \sum_{k=1}^{N}\left(d_{k}-f\left(W^{T} X_{k}\right)\right)^{2}$. However, the nonlinearity helps classification by bending the regression hyperplane and fitting it to the desired target classes. 
Output Nonlinearities: the popular nonlinearities are

- Hyperbolic tangent $(\tanh )$

$$
f(x)=\tanh (x)=\frac{e^{x}-e^{x}}{e^{x}+e^{-x}} \text {, and } f^{\prime}=0.5\left(1-f^{2}\right) .
$$

- Sigmoid (logistic)

$$
f(x)=\frac{1}{1+e^{-\alpha x}} \text {, and } f^{\prime}=\alpha f(1-f) .
$$

- Hard limit (threshold)

$$
f(x)=\left\{\begin{array}{rr}
1 & x \geq 0 \\
-1 & x<0
\end{array}, \text { and } f^{\prime}\right. \text { does not exist. }
$$

The last nonlinearity creates the Mcculloch-Pitts $(M-P)$ neurons, whereas the first two form the Modified $M-P$ neurons. The tanh and logistic functions have derivatives that are easy to compute. From now on by a neuron or node we mean a modified M-P neuron, unless stated otherwise.

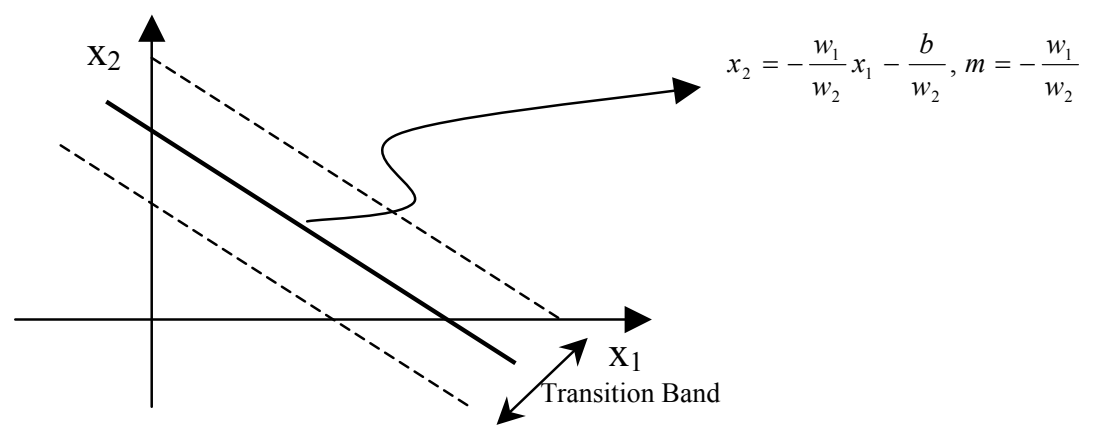

Figure 7 In modified Mcculloch-Pitts neurons class boundary depends on the weight ratios whereas the transition band depends on the actual weight values. 
Note that the intersections of decision surfaces for neurons with smooth nonlinearities create curves instead of piece-wise linear boundaries of hard thresholding.

The choice of sigmoidal functions also has biological basis. A single neuron's firing rate vs. its excitation voltage nonlinearly saturates to an upper bound that is inversely proportional to its refractory period. Furthermore, from an averaging viewpoint, if one considers the firing threshold of each cell to have a random value, an ensemble's firing threshold will have a Gaussian probability density function $p(t)$. Thus on the average, the probability of a cell firing for a stimulus of $v$ volts is $P(v)=\int_{-\infty}^{v} p(t) d t$, also called the error function which has a general sigmoidal form ${ }^{47}$. Sigmoidal nonlinearities can also create competition between the neurons of a network ${ }^{48}$.

\section{Training Algorithms}

Here the most famous architecture for static nonlinear ANNs that is multiple layer perceptron (MLP) will be introduced and some related supervised training algorithms will be discussed.

\section{Multi-Layer Networks}

Multi-layer nonlinear ANNs are much more powerful than their single layer counterparts. They can realize any decision surface. A two-layer network with $\mathrm{k}$ hidden

neurons can create $2^{\mathrm{k}}$ half-spaces in the input space that are then combined into decision regions by the output layer nodes. For instance, a two input ANN with three or more nodes in hidden layer can create a closed area in input space (see figure 8). One hidden layer MLP with sigmoidal activation function and a large enough number of neurons in 
the hidden layer is a universal mapper capable of approximating any continuous decision region according to Kolmogorov's theorem ${ }^{49}$.

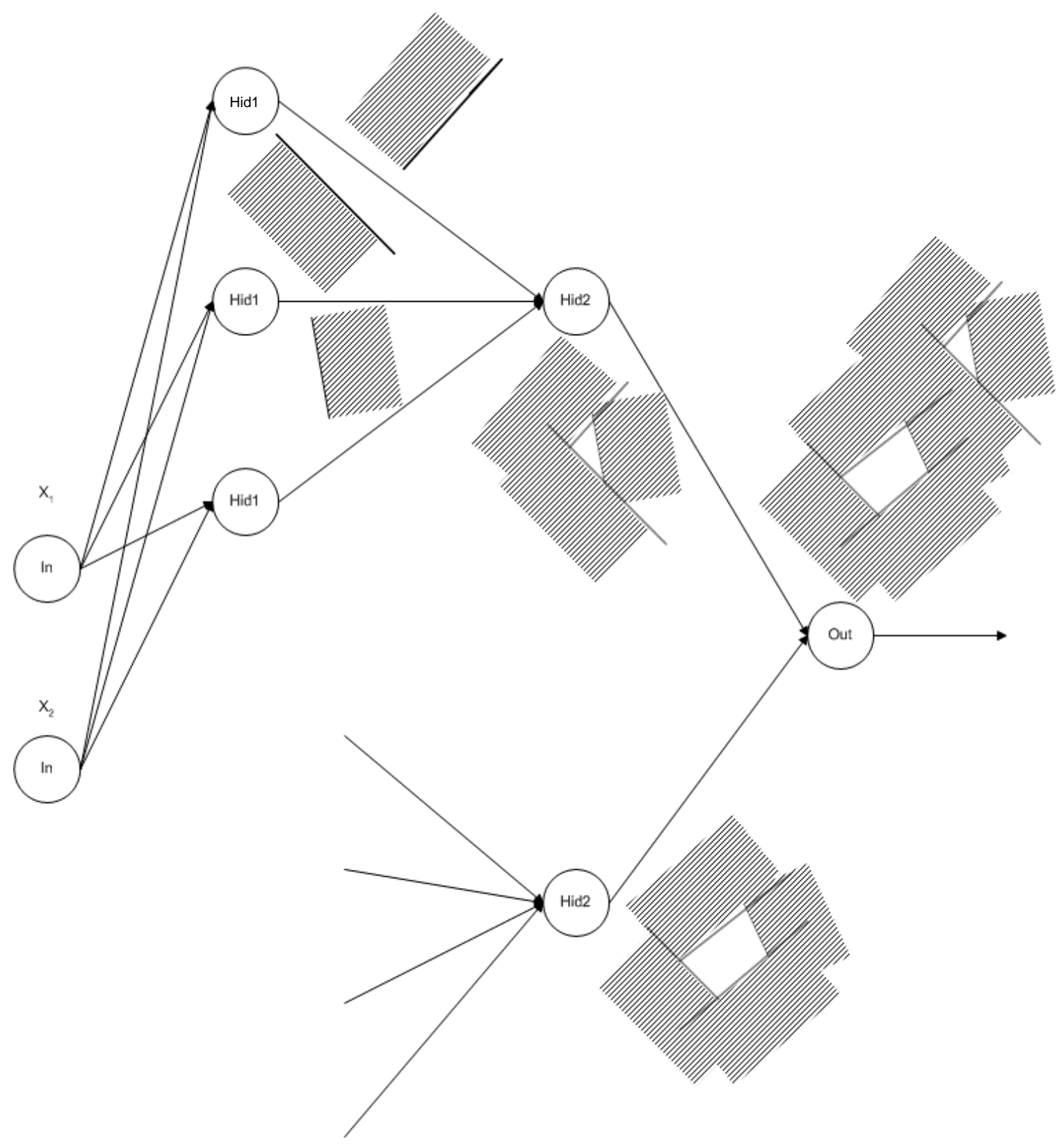

Figure 8 MLPs can create arbitrary convex decision surfaces. 
For two hidden layers, the discriminant function takes the form of $y=f_{\text {out }}\left(\Sigma f_{2 n d-h i d}\left(\Sigma f_{\text {Ist-hid }}(\right.\right.$ inputs $\left.\left.)\right)\right)$ with $f_{\text {lst-hid }}$ creating the hyperplanes in the input space, $f_{\text {2nd-hid }}$ combining those hyperplanes into disjoint areas, and $f_{\text {out }}$ combining these disjoint decision enclosures.

The two hidden layer MLP is also a universal approximator. Despite being slower in adaptation, it is more versatile. However, a one hidden layer MLP can asymptotically approximate the performance of a 2 hidden layer MLP when the number of neurons in the hidden layer approaches infinity.

Back-propagation for Multiple Hidden Layer MLP: first let's define the notation []$_{i}^{l}(n)$, where the subscript $i$ represents the node number within a layer, the superscript $l$ the layer number $l=1,2, \ldots L$, and $n$ the iteration number. The training data is given as $\left\{\left(\vec{X}_{p}, \vec{D}_{p}\right)\right\}$ where the $\mathrm{p}^{\text {th }}$ input-output training pair $\left(\vec{X}_{p}, \vec{D}_{p}\right)$ is presented as $\vec{X}=\left[\begin{array}{c}x_{0} \\ x_{1} \\ x_{2} \\ \vdots\end{array}\right]=\left[\begin{array}{c}y_{0}^{0} \\ y_{1}^{0} \\ y_{2}^{0} \\ \vdots\end{array}\right], \vec{D}=\left[\begin{array}{c}d_{1} \\ d_{2} \\ d_{3} \\ \vdots\end{array}\right] ; w_{0}=$ bias,$x_{0}=1$. In a left to right network visualization, $l=0$ denotes the input layer (so $y_{i}^{0}=x_{i}$ ), $l=1$ denotes the first hidden layer and so on, till $l=L$ that denotes the output layer. For any node, say $j$ in layer $l, w_{j 0}^{l}=b_{j}^{l}$ and $y_{0}^{l-1}=1$, representing the bias term (see figure 9). Here the indices $i, j$, and $k$ are used for consecutive layer $1-1,1$, and $1+1$, respectively to show a typical three layer slice of an MLP.

The local error (or injected error) $\delta$ for the $\mathrm{j}^{\text {th }} \mathrm{PE}$ in the $\mathrm{t}^{\text {th }}$ layer at the $\mathrm{n}^{\text {th }}$ iteration is defined as

$$
\delta_{j}^{l}(n)=\varepsilon_{j}^{l}(n) f^{\prime}\left(n e t_{j}^{l}(n)\right)
$$


where

$$
\begin{gathered}
\mathcal{E}_{j}^{l}(n)= \begin{cases}d_{j}(n)-y_{j}^{L}(n) & \text { if } l=L(\text { output layer }) \\
\sum_{k} w_{k j}^{l}(n) \delta_{k}^{l+1}(n) & \text { otherwise }\end{cases} \\
n \operatorname{nt}_{j}^{l}(n)=\sum_{i} w_{j i}^{l}(n) y_{i}^{l-1}(n) \quad(\mathrm{B} 42)
\end{gathered}
$$

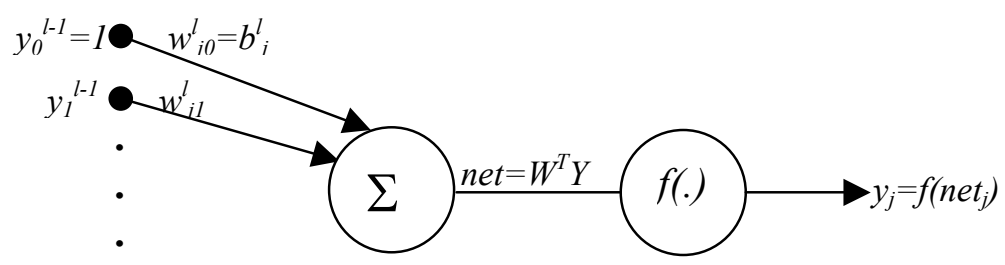

Figure 9 Node notations used in multiple hidden layer MLP back-propagation.

Using the chain formula for LMS, the backpropagation algorithm for the multiple hidden layer MLP per each iteration $n$ then can be expressed as follows.

1. Forward activation: compute output of each node, from network input to output.

$$
y_{j}^{l}(n)=f\left(n e t_{j}^{l}(n)\right), \quad n e t_{j}^{l}(n)=\sum_{i} w_{j i}^{l}(n) y_{i}^{l-1}(n)
$$

2. Back-propagated error: Compute local (injected) error $\delta$ for each node, from network output to input.

$\delta_{j}^{l}(n)=\varepsilon_{j}^{l}(n) f^{\prime}\left(\right.$ net $\left._{j}^{l}(n)\right) \quad$ where $\varepsilon_{j}^{l}(n)=\left\{\begin{array}{l}d_{j}(n)-y_{j}^{L}(n) \quad \text { if l } l=L(\text { output layer }) \\ \sum_{k} w_{k j}^{l}(n) \delta_{k}^{l+1}(n) \quad \text { otherwise }\end{array}\right.$ 
3. Update weights:

$$
w_{j i}^{l}(n+1)=w_{j i}^{l}(n)+\Delta w_{j i}^{l}(n) \quad \text { where } \Delta w_{j i}^{l}(n)=\eta \delta_{j}^{l}(n) y_{i}^{l-1}
$$

$\eta$ is the learning rate (step size). The vector form for the above equation (for weight vector going to $j^{\text {th }} \mathrm{PE}$ in the $1^{\text {th }}$ layer) can be written as:

$$
\vec{W}_{j}^{l}(n+1)=\vec{W}_{j}^{l}(n)+\Delta \vec{W}_{j}^{l}(n) \quad \text { where } \Delta \vec{W}_{j}^{l}(n)=\eta \delta_{j}^{l}(n) Y^{l-1}
$$

Note that $\vec{\nabla} J_{W_{j}^{l}}(n)=-\delta_{j}^{l}(n) Y^{l-1}$, so $\Delta \vec{W}_{j}^{l}(n)=-\eta \vec{\nabla} J_{W_{j}^{l}}(n)=\eta \delta_{j}^{l}(n) Y^{l-1}$. A general derivation that is also applicable to the temporal neural networks is given below.

\section{Computation of Gradients in Ordered Networks}

Paul Werbos ${ }^{50}$ introduced the powerful notion of ordered derivatives for calculation of sensitivities in ordered networks, which befits many types of neural networks including temporal. Here this method is introduced and the back propagation equations in feed forward MLPs are derived through the general framework of ordered networks.

Ordered Networks: An ordered network is network whose state variables can be computed in a specific order, one at a time. One can number the nodes in such a network according to their order of evaluation. A change in any state will ripple through the network according to this order and state updates can be calculated accordingly. In such networks, dependence of the sensitivity (derivative) of a variable with respect to a preceding variable can be divided into two parts: 
- Explicit or direct,

- Implicit or indirect.

Computation of sensitivities through such grouping of dependencies is the basis of ordered derivative. For instance, consider the following three-node ordered network with linear neurons. Sensitivity of $y_{3}$ with respect to $y_{1}$ in terms of ordered derivative is computed as

$$
\frac{\partial^{\text {ord }} y_{3}}{\partial y_{1}}=\frac{\partial^{\text {dir }} y_{3}}{\partial y_{1}}+\frac{\partial^{\text {ind }} y_{3}}{\partial y_{1}}=\frac{\partial y_{3}}{\partial y_{1}}+\frac{\partial y_{3}}{\partial y_{2}} \frac{\partial y_{2}}{\partial y_{1}}=w_{31}+w_{32} w_{21}
$$
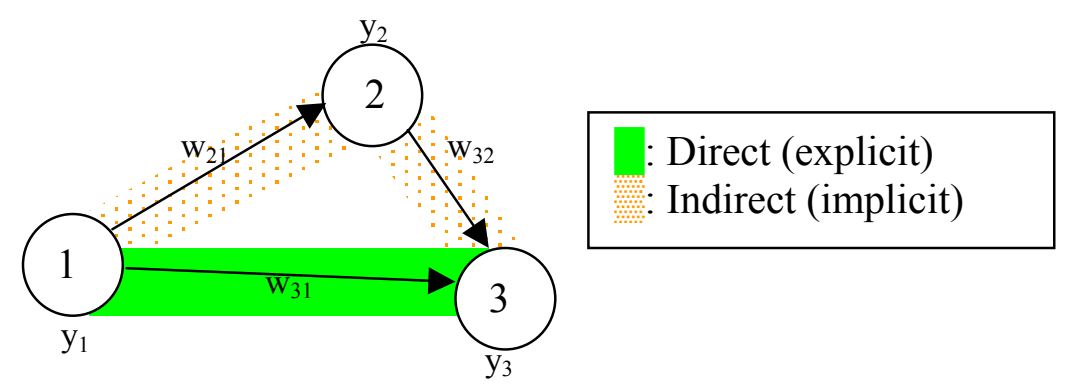

Figure 10 A snippet of an ordered network.

The superscripts $o r d$, dir, and ind indicate ordered, direct, and indirect derivatives, respectively. This is similar to the partial derivative of a multivariate function. For instance consider $y_{3}=f\left(y_{1}, y_{2}\right)$, whose dependencies are the same as those depicted in figure 10 . The multivariate derivative can be written as

$$
\begin{gathered}
d f=d y_{3}=d f_{y_{1}}+d f_{y_{2}}=\frac{\partial y_{3}}{\partial y_{1}} d y_{1}+\frac{\partial y_{3}}{\partial y_{2}} d y_{2} \\
\frac{d y_{3}}{d y_{1}}=\frac{\partial y_{3}}{\partial y_{1}}+\frac{\partial y_{3}}{\partial y_{2}} \frac{d y_{2}}{d y_{1}}
\end{gathered}
$$


Note that the state of the node $j\left(y_{j}\right)$ cannot be computed unless the states of the variables such as $y_{i}$ that $y_{j}$ is dependent on are already known, either directly on indirectly $(i<j$ in feed forward networks).

In general, for an ordered network one can arrange the node states in the order that they affect each other's updates and write:

$$
\frac{\partial^{\text {ord }} J}{\partial y_{i}}=\frac{\partial^{\text {dir }} J}{\partial y_{i}}+\frac{\partial^{i n d} J}{\partial y_{i}}=\frac{\partial^{\text {dir }} J}{\partial y_{i}}+\sum_{j>i} \frac{\partial J^{\text {ord }}}{\partial y_{j}} \frac{\partial^{\text {dir }} y_{j}}{\partial y_{i}}
$$

where $J$ is the dependent variable of interest.

In a feed forward neural network, this will be the network error (sum of output node errors $J_{i}$ over all training patterns), subject to minimization through gradient descent $J=\frac{1}{N} \sum_{i} J_{i}, \quad J_{i}=\frac{1}{2} \varepsilon_{i}^{2}=\frac{1}{2}\left(d_{i}-y_{i}\right)^{2}, \quad \frac{\partial^{d i r} J}{\partial y_{i}}=-\varepsilon_{i} \quad$ (Summation through pattern indices $\mathrm{p}$ has been omitted for clarity). One can consider $J$ in the above format as the output of another node receiving its inputs from $y_{i}$ and $d_{i}$. Note that in (B47) $\frac{\partial^{d i r} J}{\partial y_{i}}$ is zero unless $\mathrm{y}_{\mathrm{i}}$ is an output node. $\frac{\partial^{d i r} y_{j}}{\partial y_{i}}$ in $\sum_{j>i} \frac{\partial J^{\text {ord }}}{\partial y_{j}} \frac{\partial^{d i r} y_{j}}{\partial y_{i}}$ is also zero for any $\mathrm{y}_{\mathrm{j}}$ that is not directly connected to $\mathrm{y}_{\mathrm{i}}$, so the term will be reduced to direct derivative(s) of whatever node(s) $y_{j}$ that are connected to $y_{i}$ on the path from output to $y_{i}$ times the ordered derivative of $J$ vs. $y_{j}$ (backward in terms of indices). Consequently the summation index is only for the nodes $\mathrm{j}>\mathrm{i}$ since these intermediate nodes are "after" the origin node $y_{\mathrm{i}}$. In other words, in such an ordered feed forward configuration $\frac{\partial^{d i r} y_{j}}{\partial y_{i}}=0$ for $\mathrm{i}>\mathrm{j}$, which means that $\mathrm{y}_{\mathrm{j}}$ changes with respect to $\mathrm{y}_{\mathrm{i}}$ and not the other way around. By the same token, replacing $\mathrm{y}_{\mathrm{i}}$ with $\mathrm{w}_{\mathrm{ji}}$, one can write: 


$$
\frac{\partial^{o r d} J}{\partial w_{j i}}=\frac{\partial^{d i r} J}{\partial w_{j i}}+\frac{\partial^{i n d} J}{\partial w_{j i}}=\frac{\partial^{d i r} J}{\partial w_{j i}}+\sum_{k} \frac{\partial J^{o r d}}{\partial y_{k}} \frac{\partial^{d i r} y_{k}}{\partial w_{j i}}
$$

The first term $\frac{\partial^{d i r} J}{\partial w_{j i}}$ is zero since $\mathrm{J}$, if considered as a node, receives its direct inputs from $y_{\mathrm{i}}$ and $\mathrm{d}_{\mathrm{i}}$ and not the connection weights. For the indirect part $\sum_{k} \frac{\partial J^{\text {ord }}}{\partial y_{k}} \frac{\partial^{d i r} y_{k}}{\partial w_{j i}}$, the non-zero term will be for the intermediate node $\mathrm{k}=\mathrm{j}$, since this is the only node connected directly to $\mathrm{w}_{\mathrm{ji}}$. Hence, (B48) will be reduced to:

$$
\frac{\partial^{\text {ord }} J}{\partial w_{j i}}=\frac{\partial J^{\text {ord }}}{\partial y_{j}} \frac{\partial^{\text {dir }} y_{j}}{\partial w_{j i}}=\frac{\partial J^{\text {ord }}}{\partial y_{j}} \frac{\partial f\left(\text { net }_{j}\right)}{\partial w_{j i}}=\frac{\partial J^{\text {ord }}}{\partial y_{j}} f^{\prime}\left(\text { net }_{j}\right) y_{i}
$$

Now one can derive the backpropagation formulas with ordered derivatives. Starting from (B47):

Explicit (direct) term:

$$
\frac{\partial^{d i r} J}{\partial y_{i}}=\left\{\begin{array}{cl}
-\varepsilon_{i} & y_{i} \text { is output } \\
0 & \text { otherwise }
\end{array}\right.
$$

For $\frac{\partial J^{\text {ord }}}{\partial y_{j}}$, the first part of the implicit term in (B47) as well as the first term in (B49), we define

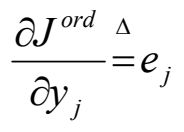


The ordered derivative of $J$ with respect to $y_{i}$ reduces to $-\varepsilon_{i}$ for the output layer $\left(e_{i}=-\varepsilon_{i}\right)$ since $J=\frac{1}{N} \sum_{j} \varepsilon_{i}^{2}, \varepsilon_{i}^{2}=\frac{1}{2}\left(d_{i}-y_{i}\right)^{2}$, and with $\mathrm{y}_{\mathrm{i}}$ having only direct ordered connection to $\mathrm{J} \frac{\partial^{\text {ord }} J}{\partial y_{i}}=\frac{\partial^{\text {dir }} J}{\partial y_{i}}+\frac{\partial^{\text {ind }} J}{\partial y_{i}}=\frac{\partial^{\text {dir } J}}{\partial y_{i}}+0=-\varepsilon_{i}$. Alternatively, one can say since the term $\frac{\partial^{\text {ind }} J}{\partial y_{i}}=\sum_{j>i} \frac{\partial J^{\text {ord }}}{\partial y_{j}} \frac{\partial^{\text {dir }} y_{j}}{\partial y_{i}}$ and for the output nodes $\mathrm{y}_{\mathrm{i}}$, there are no other nodes $j$ to the right $(\neg \exists \mathrm{j}>\mathrm{i})$, then the summation vanishes and reduces to zero.

$$
\begin{aligned}
& \text { For } \frac{\partial^{d i r} y_{j}}{\partial y_{i}} \text { part of the implicit term in (B47): } \\
& \qquad \frac{\partial^{d i r} y_{j}}{\partial y_{i}}=\frac{\partial y_{j}}{\partial n e t_{j}} \frac{\partial n e t_{j}}{\partial y_{i}}=f^{\prime}\left(n e t_{j}\right) w_{j i}
\end{aligned}
$$

As stated earlier, this term is calculated for the nodes $y_{j}$ directly connected to $y_{i}(j>i)$, otherwise the term will be zero (or connection weight is zero).

We also define

$$
\delta_{i} \stackrel{\Delta}{=} \frac{\partial^{\text {ord }} J}{\partial n e t_{i}}=\frac{\partial^{\text {ord }} J}{y_{i}} \frac{\partial y_{i}}{\partial n e t_{i}}=e_{i} f^{\prime}\left(\text { net }_{i}\right)
$$

We used (B51) in the substitution above.

Now, we can re-write our main equations (B47) and (B49). For (B47), using (B50), (B51), and (B52) we can write

(a) $y_{i}$ is an output: 


$$
\begin{gathered}
e_{i}=\frac{\partial^{\text {ord }} J}{\partial y_{i}}=\frac{\partial^{\text {dir }} J}{\partial y_{i}}+\frac{\partial^{\text {ind }} J}{\partial y_{i}}=-\varepsilon_{i}+0=-\varepsilon_{i} \\
\delta_{i}=e_{i} f^{\prime}\left(\text { net }_{i}\right)=-\varepsilon_{i} f^{\prime}\left(\text { net }_{i}\right)
\end{gathered}
$$

(b) $y_{i}$ is intermediate (not an output):

$$
\begin{gathered}
e_{i}=\frac{\partial^{\text {ord }} J}{\partial y_{i}}=\frac{\partial^{\text {dir }} J}{\partial y_{i}}+\frac{\partial^{\text {ind }} J}{\partial y_{i}}=\frac{\partial^{\text {dir }} J}{\partial y_{i}}+\sum_{j>i} \frac{\partial J^{\text {ord }}}{\partial y_{j}} \frac{\partial^{\text {dir }} y_{j}}{\partial y_{i}}=0+\sum_{j>i} e_{j} f^{\prime}\left(n e t_{j}\right) w_{j i}=\sum_{j>i} \delta_{j} w_{j i} \\
\delta_{i}=e_{i} f^{\prime}\left(\text { net }_{i}\right)=f^{\prime}\left(\text { net }_{i}\right) \sum_{j>i} \delta_{j} w_{j i} \quad \text { (B57) }
\end{gathered}
$$

In either (a) or (b), using (B51) and (B53) we can write (B49) as

$$
\frac{\partial^{\text {ord }} J}{\partial w_{j i}}=\frac{\partial J^{\text {ord }}}{\partial y_{j}} f^{\prime}\left(\text { net }_{j}\right) y_{i}=e_{j} f^{\prime}\left(\text { net }_{j}\right) y_{i}=\delta_{j} y_{i}
$$

Which is for the direct connection between $\mathrm{y}_{\mathrm{i}}$ and $\mathrm{y}_{\mathrm{j}}$ by $\mathrm{w}_{\mathrm{ji}}$.

One can observe that (B58) yields the error gradient vs. connection weights necessary for the gradient descent algorithm. (B55) and (B57) provide the required local (or injected) error for (B58). This error computation starts from the output and propagates back to the input (i.e. backpropagation) because of constraint $j>i$ in computation of inject errors in (B57).

In summary, for each iteration $n$ :

1. Compute forward activations using 


$$
y_{j}=f\left(\sum_{i<j} w_{j i} y_{i}\right)
$$

Direct inputs to node $\mathrm{j}$ can be considered as a $\mathrm{y}_{\mathrm{i}}$ from a preceding node (e.g. a sensor). The activations will be computed from input to output, according to ascending (forward) node indices.

2. Compute backward local errors $\delta_{\mathrm{i}}$ using (B55) and (B57):

$$
\delta_{i}=\left\{\begin{array}{lr}
-\varepsilon_{i} f^{\prime}\left(\text { net }_{i}\right) & \text { i is output } \\
f^{\prime}\left(\text { net }_{i}\right) \sum_{j>i} \delta_{j} w_{j i} & \text { otherwise }
\end{array}\right.
$$

These injected errors will be computed from the output to the input, i.e. according to descending (backward) node indices. net values(e.g. net $\left.{ }_{j}=\sum_{i<j} y_{i} w_{j i}\right)$ are already known from step 1 .

3. Compute weight updates for next iteration $n+1$ using (B58) and substituting results from steps 1 and 2:

$$
\begin{gathered}
\frac{\partial J}{\partial w_{j i}}=\delta_{j} y_{i} \\
w_{j i}(n+1)=w_{j i}(n)-\eta \frac{\partial J}{\partial w_{j i}}(n)
\end{gathered}
$$

4. Proceed to the iteration $\mathrm{n}+1$, using inputs from the current pattern $\vec{X}_{p}$ or the next pattern $\vec{X}_{p+1}$. 
Computational Complexity of Backpropagation: Both the forward (activation, $\left.y_{j}=f\left(\sum_{i<j} w_{j i} y_{i}\right)\right)$ and the backward (error, $\delta_{i}=f^{\prime}\left(\right.$ net $\left.\left._{i}\right) \sum_{j>i} \delta_{j} w_{j i}\right)$ paths of a network with $\mathrm{N}$ nodes, in terms of number of multiplication, have the asymptotic computational complexity of $\mathrm{O}\left(\mathrm{N}^{2}\right)$. This is because the $\mathrm{N}$ nodes can have a maximum of $\left(\begin{array}{l}N \\ 2\end{array}\right)=\frac{N(N-2)}{2}$ connections, with a connection weight multiplication associated to each.

Design of MLPs is very dependent on the choice of topology. Too few hidden layers may not be able to solve the problem (e.g. the XOR problem which needs at least one hidden layer), while too many hidden layers can cause extra computation burden, and much worse, create spurious regions that are "don't care" for training but not necessarily for test sets (bias-variance dilemma). GETnet addresses this problem by minimizing the network size through its evolutionary MDL network design (please see section C).

A well-trained and well-designed MLP with $\mathrm{L}_{2}$ error criterion can yield $a$ posteriori probability of the desired target values given the observed input values, so it can construct an optimal Bayesian classifier.

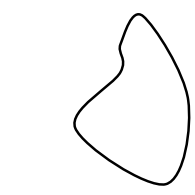

Original decision region

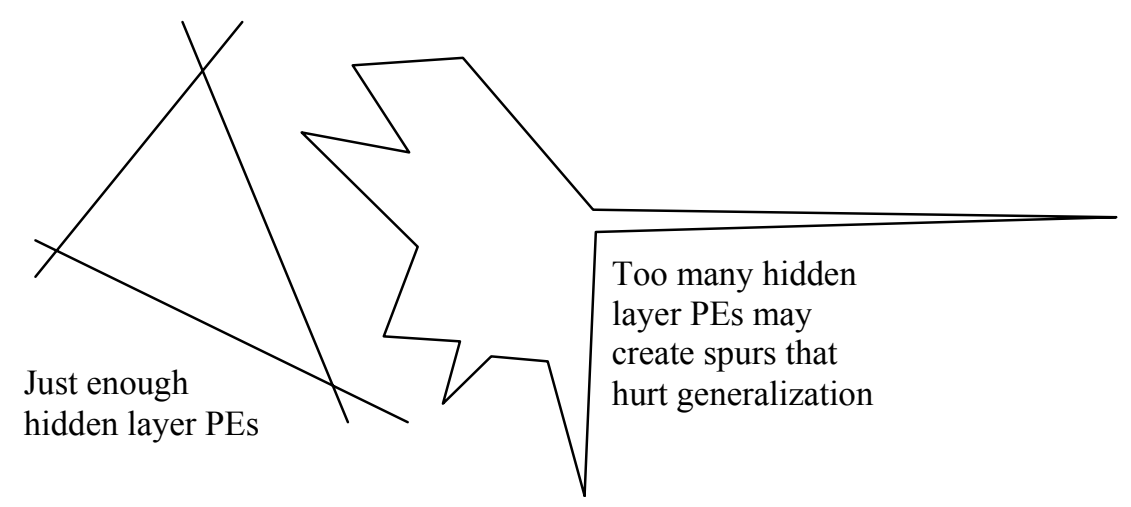

Figure 11 The problem of choosing the right number of hidden units. 


\section{Improving Backpropagation Learning}

The simple backpropagation algorithm introduced earlier has many advantages such as simplicity, locality, and online implementation. Nevertheless, it can be improved to avoid more or less situations such as stalling on flat regions of the error surface, local minima, etc. Some of the human-tuned improvement techniques are as follows. Please note how many parameters have to be guessed by the designer with no given definite analytical guideline, further demonstrating the baby-sitting problem.

Momentum learning: Consider a hypothetical weight track such as the one depicted in figure 12, where the network under training is rolling down under an imaginary gravity and surface friction. Then each weight such as $\mathrm{w}_{\mathrm{ji}}$ will not only change because of the error gradient, but also the gained momentum under the imaginary gravitational acceleration. To incorporate this concept, (B61) can be augmented with a fraction of last weight change as

$$
w_{j i}(n+1)=w_{j i}(n)+G_{j}(n)+\alpha\left(w_{j i}(n)-w_{j i}(n-1)\right)
$$

where $\alpha$ is the momentum constant, usually between 0.5 and 0.9 and chosen manually, and $\mathrm{G} \mathrm{j}$ is the gradient descent update term $G_{j}=-\eta \frac{\partial J}{\partial w_{j i}}=-\eta \delta_{j} y_{i}$.

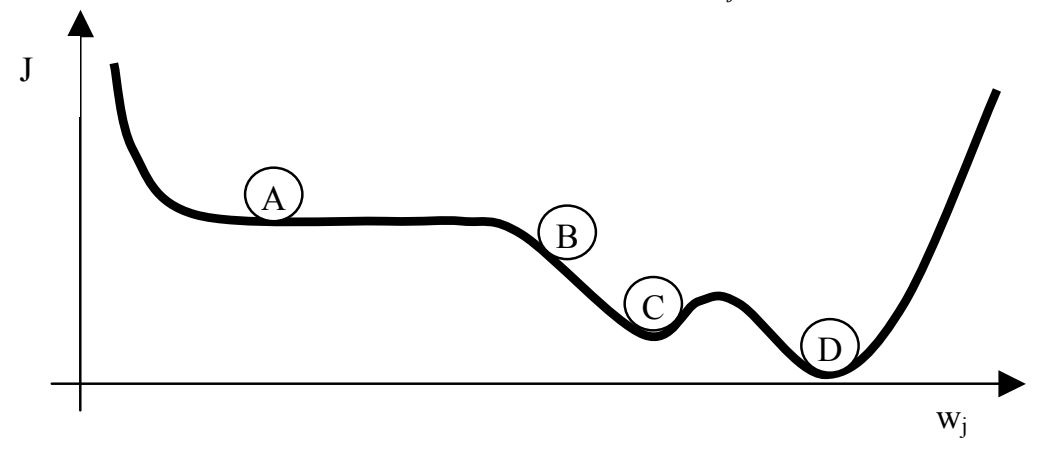

Figure 12 An imaginary error surface with local minimum and a plateau to demonstrate momentum learning. 
This way if the network hits a finite plateau (A in figure 12), the gained momentum, depending on the value of $\alpha$, can push the operating point further to the next downhill (point B), if any. Furthermore, momentum can move the network out of shallow minima (point C). This way the network can hopefully find a better next minimum (point D).

Adaptive Step Size: for problems such as linear regression, the step size should be adjusted according to the eigenvalues in each direction. One can also adjust the step size for a faster and more stable learning by using simple heuristics such as increasing the step size when the learning curve is flat and decreasing it if the learning network starts to oscillate around a minimum. This observation should be made for each connection's step size. As long as two successive weight updates $-\eta_{j i} \frac{\partial J}{\partial w_{j i}}$ (or the last gradient vs. a running sum of previous gradients) have the same sign the step size for that particular weight should be increased, and if the sign for a weight toggles, then the corresponding step size should be decreased. One can increase the learning rate for each connection by a small constant per iteration for a linear and slow step size growth. If the learning rate is too high, it can be decreased by a fraction of the previous step size for geometric and fast reduction. This step size adaptation algorithm is called Delta-bar-Delta, and is expressed mathematically as

$$
\eta_{j i}(n+1)= \begin{cases}\eta_{j i}(n)+a & \text { if } \frac{\partial J(n)}{\partial w_{j i}}\left(\gamma \frac{\partial J(n-1)}{\partial w_{j i}}+\sum_{m=1}^{n-2}(1-\gamma)^{n-m-1} \frac{\partial J(m)}{\partial w_{j i}}\right)>0 \\ b \eta_{j i}(n) & \text { if } \frac{\partial J(n)}{\partial w_{j i}}\left(\gamma \frac{\partial J(n-1)}{\partial w_{j i}}+\sum_{m=1}^{n-2}(1-\gamma)^{n-m-1} \frac{\partial J(m)}{\partial w_{j i}}\right)<0 \\ \eta_{j i}(n) & \text { if } \frac{\partial J(n)}{\partial w_{j i}}\left(\gamma \frac{\partial J(n-1)}{\partial w_{j i}}+\sum_{m=1}^{n-2}(1-\gamma)^{n-m-1} \frac{\partial J(m)}{\partial w_{j i}}\right)=0\end{cases}
$$

$0<\gamma \leq 1$ and is set manually to determine contribution of previous gradients' history. For instance, $\gamma=1$ only compares the previous gradient to the current. $0<b<1$ for 
step reduction and should be set manually as well. Variations of this scheme such as Almeida $^{51}$ or Fahlman ${ }^{52}$ are also used utilized.

One other method for step size control is through scheduling. The error criterion is a function of the network's adaptive parameters, here the weight vector; W. If the direction of search in the adaptive parameter space, e.g. the fastest local descent $-\nabla \mathrm{J}(\mathbf{W})$ is denoted by $\mathbf{S}$, then we desire to find the best step size $\eta$ in order to have the fastest possible descent to the error minimum for each step $\mathrm{k}$ by minimizing $\mathrm{J}\left(\mathbf{W}_{\mathrm{k}}+\eta_{\mathrm{k}} \mathbf{S}_{\mathrm{k}}\right)$. One may be able to find an analytical solution for the best step size, but usually for more complicated networks one should use a heuristic such as scheduling or use trial and error, with the tradeoff being between speed (bigger step size) and accuracy (smaller step size). One popular learning rate scheduling is method is simulated annealing ${ }^{53}$. In this method, learning will start with bigger step sizes to enjoy initial speed (provided that the network is not initially near its goal) and then later, when the network is nearer to an error minimum, the step size is decreased to achieve greater accuracy. One formulation for this scheduling can be written as:

$$
\eta_{k}=\frac{\eta_{1}}{1+\frac{k-1}{n_{0}}}
$$

Where $\eta_{1}$ is the initial step size, $\mathrm{k}$ the iteration number. Constants $\eta_{1}$ and $n_{0}$ should be set experimentally by the designer.

Another issue that can be helped through step size is to adjust for the nonlinearity attenuation of back-propagated error. According to (B60) $\delta_{j}=f^{\prime}\left(n e t_{j}\right) \sum_{k>j} \delta_{k} w_{k j}$, which determines the amount of weight update as expressed in (B61) $\Delta w_{j i}=-\eta \delta_{j} y_{i}$. However, for sigmoidal activation function $f^{\prime}=f(1-f)$ which is always smaller than 1 , so the 
weighted sum of errors from the next layer $\sum_{k>j} \delta_{k} w_{k j}$ is always attenuated by the factor $f^{\prime}($ net $)$.

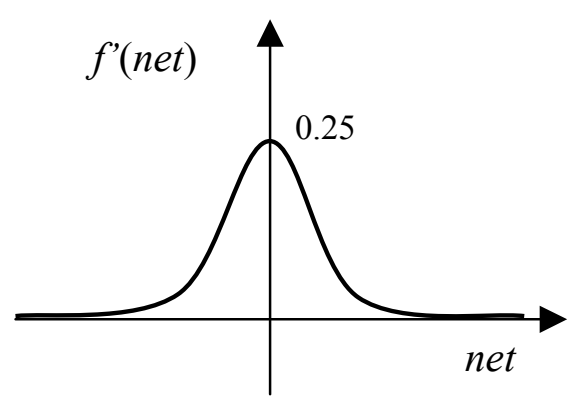

Figure 13 Derivative of the sigmoid function has a maximum of 0.25 at the origin.

This will make the weight update $\Delta w_{j i}=-\eta \delta_{j} y_{i}$ shrink from the output to the input layers, so the first layer's adaptation may become very sluggish. One remedy is increasing the step size $\eta$ from output layer to the input layer, with the ratio determined manually. The rule of thumb is increasing $\eta 2$ to 5 times, from each layer to the one preceding it.

Random Perturbation During Learning: borrowing from the idea of simulated annealing, one can add random perturbation (usually zero-mean noise) to the adapting parameters (e.g. weights) during the learning period to move them out of local minima or plateaus. This noise can be scheduled so it would become negligible during final iterations, when the network is hopefully converging to the desired goal. This is one of the motivations behind weight perturbations in GETnet, as explained later in section C. However, in GETnet the Baldwin effect replaces the scheduling for reduction of perturbation.

Initial Conditions: assigning initial weights to nonlinear MLPs is an important issue, since the starting point for training should not be far from the intended goal. This is not an issue for linear networks since their MSE error surface is hyperbolic with a unique 
minimum, whereas nonlinear neural networks can have multiple, non-global minima on their performance surfaces. An undesirable initial condition can also lead to undesirable local minima, slow convergence, or degenerate answers (such as all 0 weights in an XOR problem). Furthermore, neurons that are initialized in their linear region $(\mid$ net $\mid<<1)$ train faster since this results in higher $\Delta w_{j i}=-\eta y_{i} f^{\prime}\left(n e t_{j}\right) \sum_{k>j} \delta_{k} w_{k j}$ because of higher $f^{\prime}\left(n e t_{j}\right)$.

There are no comprehensive analytical solutions to this problem, so usually random weights in conjunction with trial and error are used. However, there are some rules of thumb for guiding the random initialization, such as Nguyen-Widrow so neurons will be initialized in a region for faster training ${ }^{54,55}$, which is used in GETnet during instantiation of new networks (please see appendix B for more details). Subsequent networks partially inherit their contents from the previous generation.

Training set size: it can be shown ${ }^{56}$ that for an MLP with $N_{W}$ weights, the number of required training data points $N$ for reaching an error $\varepsilon$, given that the training data is representative of the test data, obeys the inequality

$$
N>\frac{N_{W}}{\varepsilon}
$$

This shows a linear growth of training set with respect to network parameters, which is another advantage of MLPs compared to the other classifiers. It also shows that as a rule of thumb, for a $10 \%$ error, one needs 10 training data points per weight. However, in the real world we usually do not have such a big training set, so we might need to downsize the network by reducing the number of nodes or connection weights (sparse connectivity). One can also decrease the number of input nodes by preprocessing the data and extracting fewer features for the network input. GETnet implements these notions by competitive regularization and adaptive pruning. 


\section{Second Order Algorithms}

The introduced gradient based back propagation only uses the information of the first derivative of the cost function with respect to the adaptive parameters, with a sample-based noisy estimation of the gradient. There are other search methods that use more information from the curvature of the error surface through higher order derivatives as well as global search methods such as evolutionary algorithms. The advantage of the gradient-based back-propagation is in its simplicity (just a few additions and multiplications per weight update), dependence on local parameters, and capability of online, real time training.

The more complex second order algorithms can be derived from the Taylor approximations. Going back to the basic problem of minimizing the error function $J(\boldsymbol{W})$, its expansion by the multivariate Taylor series around the operating point $\mathbf{W}_{0}$ can be written as

$$
\begin{gathered}
J(\vec{W})=\left.\sum_{k=0}^{\infty} \frac{1}{k !}\left(\left(\vec{W}-\vec{W}_{0}\right) \cdot \vec{\nabla}_{\vec{W}}\right)^{k} J(\vec{W})\right|_{\vec{W}=\vec{W}_{0}} \quad \text { or } \\
J\left(w_{1}, w_{2}, \ldots w_{M}\right)=\left.\sum_{k=0}^{\infty} \frac{1}{k !}\left(\sum_{l=1}^{M}\left(w_{l}-w_{0 l}\right) \frac{\partial}{\partial w_{l}}\right)^{l} J\left(w_{1}, w_{2}, \ldots w_{M}\right)\right|_{\vec{W}=\vec{W}_{0}}
\end{gathered}
$$

where $\nabla_{W}$ is the gradient operator, the variable vector $\vec{W}=\sum_{l=1}^{M} w_{l} \hat{u}_{l}$ represents all the network weights in the M-dimensional weight space with unit vectors $\hat{u}_{l}$, and $\vec{W}_{0}=\sum_{l=1}^{M} w_{0 l} \hat{u}_{l}$ is the initial weight vector close to $\boldsymbol{W}$. (B66) can be obtained from the repeated integration of the $\mathrm{n}+1^{\text {th }}$ derivative of $J$ with respect to the weights: 


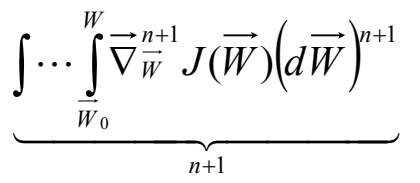

Using (B66), the second order truncated vector Taylor approximation can be written as ${ }^{57}$

$$
J(\vec{W})=J\left(\vec{W}_{0}\right)+\left(\left.\left(\vec{W}-\vec{W}_{0}\right) \cdot \vec{\nabla}_{\vec{w}} J(\vec{W})\right|_{\vec{W}=\vec{W}_{0}}\right)+\frac{1}{2}\left(\vec{W}-\vec{W}_{0}\right) \cdot\left(\left(\vec{W}-\vec{W}_{0}\right) \cdot \vec{\nabla}_{\vec{w}}\left(\left.\vec{\nabla}_{\vec{w}} J(\vec{W})\right|_{\vec{W}=\vec{W}_{0}}\right)\right.
$$

(B68) can be written in matrix notation as

$$
J(\vec{W})=J\left(\vec{W}_{0}\right)+\left(\vec{W}-\vec{W}_{0}\right)^{T} \vec{\nabla} J\left(\vec{W}_{0}\right)+\frac{1}{2}\left(\vec{W}-\vec{W}_{0}\right)^{T} H\left(\vec{W}_{0}\right)\left(\vec{W}-\vec{W}_{0}\right)+\cdots
$$

$\mathbf{W}=\left[\mathrm{w}_{\mathrm{i}}\right]_{\mathrm{M} \times 1}$ is the column matrix of all the network weights (total weight vector made of concatenation of all the nodes' weight vectors) and $\mathbf{W}_{0}=\left[\mathrm{W}_{0 \mathrm{i}}\right]_{\mathrm{M} \times 1}$ is the initial center close to $\mathbf{W} . \nabla \mathrm{J}\left(\mathbf{W}_{0}\right)$ is the gradient in the form of a column vector $\nabla \mathrm{J}(\mathbf{W})=\left[\left(\partial / \partial \mathrm{w}_{\mathrm{i}}\right) \mathbf{J}\right]_{\mathrm{M} \times 1}$ evaluated at $\mathbf{W}=\mathbf{W}_{0} . \mathbf{H}$ is the Hessian matrix of the error function $\mathrm{J}(\mathbf{W}): \mathfrak{R}^{\mathrm{M}} \rightarrow \mathfrak{R}$. The Hessian itself is a function of the network weights and defined as

$$
H(\vec{W})=\left[h_{i j}\right]_{M \times M}, \quad h_{i j}=\frac{\partial J\left(w_{1}, w_{2}, \ldots, w_{M}\right)}{\partial w_{i} \partial w_{j}}
$$

$\mathbf{H}\left(\mathbf{W}_{0}\right)$ is a symmetric matrix of partial derivatives evaluated at $\mathbf{W}_{0}$. Thus gradient of $J$ in with respect to $\mathbf{W}$ results in

$$
\nabla J(\vec{W})=\vec{\nabla} J\left(\vec{W}_{0}\right)+H\left(\vec{W}_{0}\right)\left(\vec{W}-\vec{W}_{0}\right)+\cdots
$$

The first order methods such as gradient descent use the first term, and the second order methods such as Newton use the second order approximation which involves the Hessian. 
In fact, equating the second order truncation of (B66) with zero to find coordinates of minimum error in weight space ( $\mathbf{W}$ for which $\nabla \mathrm{J}(\mathbf{W})=0$ ) results in

$$
\begin{gathered}
0=\vec{\nabla} J\left(\vec{W}_{0}\right)+H\left(\vec{W}_{0}\right)\left(\vec{W}-\vec{W}_{0}\right) \text { or } 0=H^{-1}\left(\vec{W}_{0}\right) \vec{\nabla} J\left(\vec{W}_{0}\right)+\left(\vec{W}-\vec{W}_{0}\right) \text {, yielding } \\
\vec{W}=\vec{W}_{0}-\left.\left.H^{-1}\right|_{\vec{W}_{0}} \vec{\nabla} J\right|_{\vec{W}_{0}}
\end{gathered}
$$

Which is the same as our earlier formula for the Newton method in linear ANN, since from (B15) $\vec{\nabla}_{\vec{W}} J=R W-P$ so $H=\vec{\nabla}(\vec{\nabla} J)=R$. (B72) can be used iteratively as

$$
\vec{W}(n+1)=\vec{W}(n)-\left.\left.H^{-1}\right|_{\vec{W}(n)} \vec{\nabla} J\right|_{\vec{W}(n)}
$$

Note that in both (B72) and (B73) W is considered to be a column matrix representation of the total network weight vector. The Hessian is not local as it needs non-local information (e.g. partial derivatives of $J$ with respect to all weight combinations $w_{i} w_{j}$ across the whole network), and increases quadratically in size with the number of weights which makes it computationally expensive, not to mention the computation of its inverse provided that it exists. One can either improve the first order method (e.g. line search methods) or approximate the Hessian (e.g. for the pseudo Newton methods), as described below.

Line Search: As discussed earlier in learning step size control, the goal of learning is minimizing the error function, $J(\mathbf{W})$. The direction of fastest local descent in each step is $-\nabla J(\mathbf{W}(\mathrm{n}))$, which is perpendicular to the $J=$ constant contours. Based on the eccentricity and skew of the error surface $J(\mathbf{W})$, the first order gradient search will go through a zigzag path. One can reduce this longer jagged path by combining the two most recent update directions as: 


$$
\left\{\begin{array}{c}
\vec{s}(n+1)=-\vec{\nabla} \vec{W} J(n)+\alpha(n) \vec{s}(n) \\
\Delta \vec{W}(n+1)=\eta \vec{s}(n+1)
\end{array}\right.
$$

This is called the conjugate gradient method $^{58} . \alpha$ can be determined through different methods as described in appendix A. Scaled Conjugate Gradient method, or SCG, is the method of choice used for GETnet, since it avoids a plethora of manually set constants and complexities of the other accelerated gradient searches described earlier. Please refer to section $\mathrm{C}$ for more information.

Pseudo-Newton Methods: In these methods a computationally less complex approximation to the Hessian in conjunction with (B73) is used. One method is to keep only the diagonal terms of the Hessian so (B73) can be written as

$$
w_{i}(n+1)=w_{i}(n)-\frac{\frac{\partial J(n)}{\partial w_{i}(n)}}{\frac{\partial^{2} J(n)}{\partial w_{i}(n)^{2}}}(\mathrm{~B} 75)
$$

or use the absolute value of the second derivative plus a small positive constant $c$ to avoid division by zero:

$$
w_{i}(n+1)=w_{i}(n)-\frac{\frac{\partial J(n)}{\partial w_{i}(n)}}{\left|\frac{\partial^{2} J(n)}{\partial w_{i}(n)^{2}}\right|+c}
$$

There are also better approximation methods such as Levenberg-Marquardt, DavidsonFletcher-Powell, and the Broyden-Fletcher-Goldfarb-Shanno ${ }^{59}$. 


\section{Improving Backpropagation For Unseen Data}

To improve generalization, one should find measures indicating when the network has learned the problem in a general sense. This can be done among other methods by observing the performance on a portion of the test data as the criteria to end training (to avoid overtraining and memorization), or pruning a network that has too many free parameters (to reduce don't care regions). One can also take the democratic approach and ask different classifiers to cast their votes which averages out their output errors. All of the above techniques are utilized by GETnet.

\section{Stopping the Training}

A simple criterion is using a stopping threshold for training error. For instance, one can stop training when the network error (e.g. MSE) reaches a threshold, say 0.02. However, general network errors such as MSE are indirect measures of performance and are based on the training set and do not carry information on the test set and thus generalization cannot be guaranteed. Setting too low a threshold for training set error might make the network over-fit or memorize the training or the preset threshold might never be achieved (a maximum number of iterations can be set to avoid an infinite loop in this case). Increasing the error will stop training before appropriate class boundaries are obtained. It is also possible to set a stopping threshold to the performance measure's rate of change. However this criterion still suffers from the above stated issues, plus some networks start converging to the answer after a period of low MSE slopes, in which case the network might exit training prematurely. Based on the above, it would be much better to base the stop criterion on generalization. The goal is stopping the network from overtraining, when the discriminants start to leak to the don't care areas where some of the unseen test data may reside. One can keep a portion of the training set as the crossvalidation set (usually $10 \%$ of the training data). The network should check after every few iterations to see whether the cross validation error is increasing, and stop early in the 
interest of generalization even if the training error is still decreasing (see figure 14). This method is also known as early stopping.

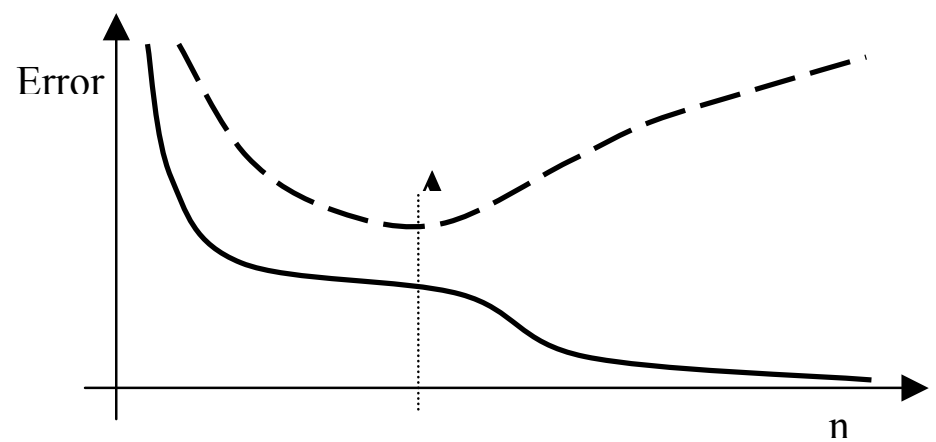

Figure 14 The network should stop early at point A for optimum overall performance on both the training (solid curve) and cross-validation data (dashed curve) and retain its generalization.

\section{Network Pruning}

Earlier the relation between network size and architecture vs. its classification capabilities (bias-variance dilemma) was discussed. Methods such as early stopping with cross validation help generalization by avoiding over-training but they do not address the problem in terms of model size and extra free parameters. One might want to follow Occam's razor principle and use a network just big enough to solve the problem at hand. To achieve an ANN of such size, one can either start from a smaller network and grow it to reach a working network, or start from a bigger ANN and downsize it by pruning the network (removing inconsequential parameters). GETnet tries to eliminate unnecessary connections while adding the new ones according to evolutionary experience, thus it is capable of both growing and shrinking the network.

Weight Decay: The idea is to decrease all the weights just a little during each iteration. If a weight was not to be decreased, the learning algorithm will increase it in next iteration. Otherwise the weight will be gradually driven to zero and eliminated after falling below a threshold. 
Finding Importance of a Parameter: a good but complicated method for finding the most suitable candidates for weight elimination is calculation of each parameter's saliency, by finding the effect of setting it to zero in the error function. It can be shown ${ }^{60}$ that the Hessian has this information and a local approximation for weight saliency can be obtained from:

$$
s_{k}=\frac{H_{k k} u_{k}{ }^{2}}{2}=\frac{1}{2} \partial u_{k}^{2} \frac{\partial^{2} J}{\partial u_{k}{ }^{2}}
$$

where $u_{k}=w_{j i}$, and $k \in\{$ all weight index pairs $\}$. To implement this method, also known as optimal brain damage, one should first train the original network, then calculate the saliency of its weights and sort them accordingly, and keep a predefined top percentage. Then the network should be retrained with this new smaller set of weights and their original initial values. This process will be repeated until the desired number of weights (based on the size of available training data set, etc) and generalization is achieved, i.e. the optimal damage (reduction) to the brain (neural network) has been found.

Another pruning technique is keeping only the most important inputs. Selection can be performed by calculating output sensitivity with respect to each input. One should first train the network and then add random perturbation to the inputs one by one and measure the resulting swing in the output(s). The sensitivity then can be found from the ratio of resulting output variance to input variance. In any case, one should always consider the negative effects of network complexity, as in the regularization term in (B9).

GETnet prunes the synaptic weights using a relative importance $(\mathrm{C} 18)$. The evolutionary part of GETnet also estimates the sensitivity of network in terms of the fitness score with respect to connections and nodes by changing them according to the strategy parameters. 


\section{Committee of Networks}

A neural network has a stochastic learning nature since each training episode results in a different set of weights. Even if the training errors of some of the runs are not minimal, they might prove to be the better solution based on their performance on the unseen test data (generalization). It was also mentioned that architecture and size influence network behavior and performance. One approach for getting a better performance is to retain all those solutions obtained from different training runs on the same network as well as different topologies and average the results, also known as committee of networks method. It can be shown that for such an approach, given that each network's error is statistically independent, the MSE error of the committee of networks can be reduced $\mathrm{N}$ times compared to the mean error of an individual network with $\mathrm{N}$ being the number of networks in the committee ${ }^{61}$. In practice the errors are higher since the errors of the networks are not independent. In the case of using one topology with different parameters in a committee, the resulting system can be viewed as a sparsely connected network, i.e. a special case of a weight-eliminated network that has resulted in parallel modules.

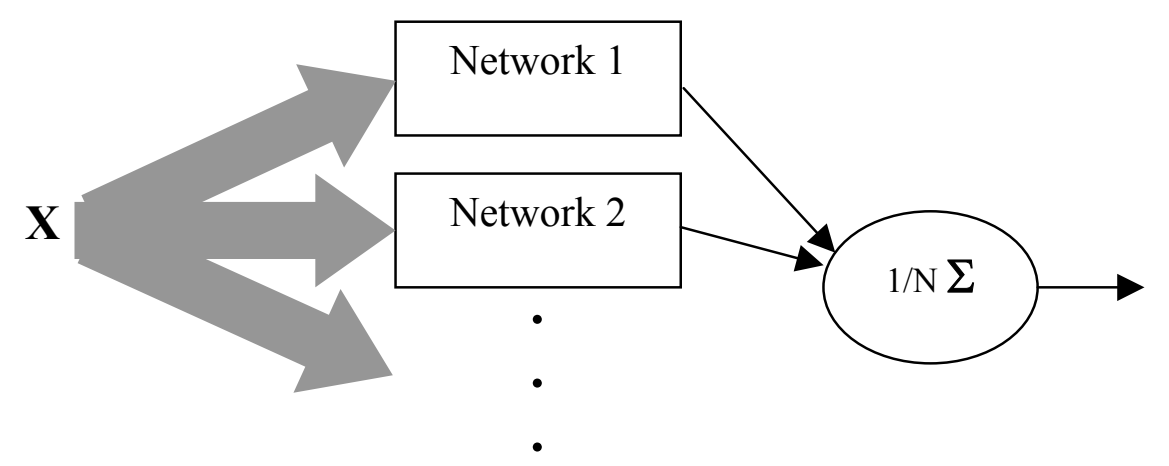

Figure 15 A committee of networks. 


\section{B3-4 Dynamic Nonlinear Neural Networks}

All the biological neural networks perceive and process information in time. Though more complicated, temporal processing can give an adaptive system a lot more information about the input sequences. Inputs with the same static distribution might have different dynamic progressions (i.e. different signal trajectories in time). For instance, a time sequence of the sum of two sinusoidal signals with different frequencies but equal amplitudes can be separated by filters, while their cumulative amplitude traces are non-separable if the time progression (frequency) information is not available. In other words, in dynamic systems the order as well as the pace of presented data is given and important, whereas in static systems it is not.

Temporal inputs along with temporal pattern processing gives rise to the notion of memory. Path delay lines (both forward and feedback) can store a moving window of the signal history and can be considered as a form of Short Term Memory (STM) ${ }^{62}$, such as the one discussed in MA model of a linear neuron. Information stored in form of connection weights of an artificial neural network such as the distributed memory of a Linear Associative Memory (LAM) ${ }^{63}$ as well as the infinite delayed feedback loops can be considered as Long Term Memory (LTM).

Dynamic systems with temporal connections can be feed-forward or have feedbacks (recurrent systems). In either case, because of time delays the output will have a transient period before reaching steady state, given that system is stable. Use of delay lines as memory structures inside the feed forward neural network provides static snapshots of the signal's past within a time window, giving rise to a Finite Impulse Response (FIR) system ${ }^{64}$. The length of the delay line must be carefully chosen to capture the desired information. If the sought features are stationary, their derivation should remain the same in spite of the sliding input time window, given enough length of the static window. 
In theory, recurrent systems offer infinite recall through feedback loops and create Infinite Impulse Response (IIR) systems. However, such systems may become unstable or oscillate, which sometimes may be desirable in neurocomputing. In fact, nodes in a recurrent neural network with sigmoidal activation function can saturate towards either output extreme, mimicking a finite-state machine. One can consider the states of such a network (i.e. outputs of the nodes) with $\mathrm{N}$ nodes being represented by a hyper $\mathrm{N}$-cube. A saturating network approaches one of the vertices, which is called an attractor. This topic is further discussed under network energy later on in this section. Computation through attractors can display regular or chaotic behavior. However, the training of such a network is much more involved. It is possible to unroll the feedback loops and simulate recurrent systems with feed forward time delay neural networks (TDNN) for a given time span, or to use temporal versions of back propagation ${ }^{65}$.

\section{Time Delay MLP (TDNN)}

If one places a delay line (such as the one used in the MA filter) at the input of a multilayer Perceptron, the resulting structure is called a focused Time Delay Neural Network, or simply a TDNN. The term focused emphasizes the fact that the short-term memory structure is focused in the input. Such structures were introduced by Waibel for speech processing ${ }^{22}$. TDNN can also be used for other nonlinear temporal mappings such as nonlinear dynamic system identification and nonlinear time series prediction. In fact, an adequate predictor can autonomously reproduce a time series (dynamic modeling). It is enough to set the right initial conditions (seeding the system) and connect the output of the adapted predictor $y(n)=f(x(n))=x(n+1)$ to its input, as shown below.

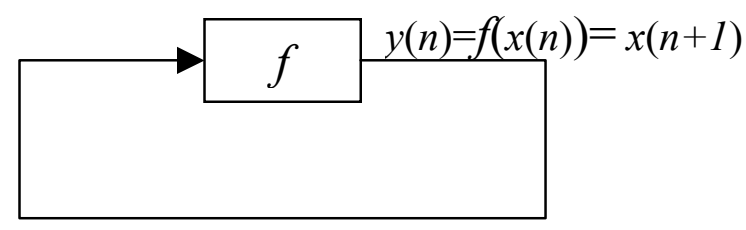

Figure 16 Dynamic modeling. 
As can be seen from figure 17, the first layer of the TDNN is essentially a bank of moving average (MA) filters with nonlinearities after each weighted time delay average. The subsequent layers simply function like regular MLPs, nonlinearly mapping the results of the various filterings of the input signals to the (desired) output signals.

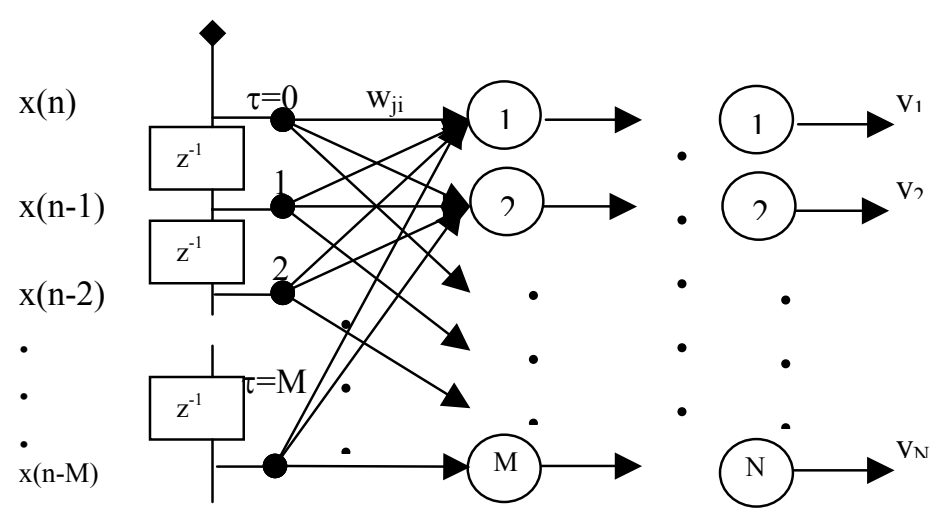

Figure 17 A focused time delay multilayer Perceptron.

In temporal paradigms the input as well as desired and network outputs will all be single or multidimensional sequences. As long as the desired output for each time step exists, the network weights can be trained with the static algorithms such as backpropagation. A TDNN with appropriate MLP topology and memory resolution and depth can be a universal temporal mapper ${ }^{66}$.

Generally speaking, the short-term memory in a neural network can be implemented either by a delay line as described earlier or by a delayed feedback connection (e.g. from the output to the input of a node) to create a recurrent element. Such elements are also known as a context node (see figure 18). The depth of the memory (time extent of impulse response) of the delay line memory kernel is equal to the length of the delay line, whereas in the case of recurrent context memory it is theoretically infinite but practically limited depending on the feedback strength. In either case, the resolution of the memory (temporal sampling grain) depends on the value of $d$, the inverse of the sampling rate of the delay element. 
The delay line STM can also be seen as a linear projector of the input signal into a space whose coordinates are the consecutive delayed values of input within the delay line. The deeper a delay line of adequate resolution, the higher the dimension of this representation. This translates into a higher chance of separation of the input patterns by the subsequent MLP since the signal trajectories will hopefully be further apart and have fewer overlaps, i.e. longer histories may potentially reveal more distinctive features.
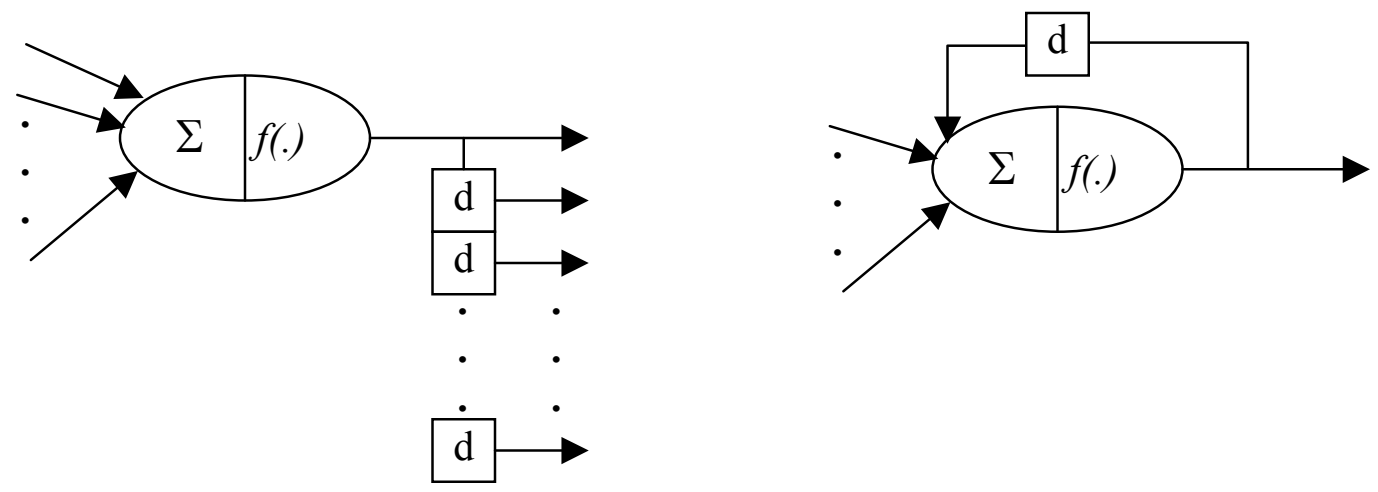

Figure 18 A delay line memory (left) vs. a recurrent or context memory (right).

A combination of the delay line memory and recurrent context memory creates a special memory system called the Gamma Memory ${ }^{6}$. Each memory element of the Gamma memory delay line is made of a simple first-order recurrent kernel (figure 19).
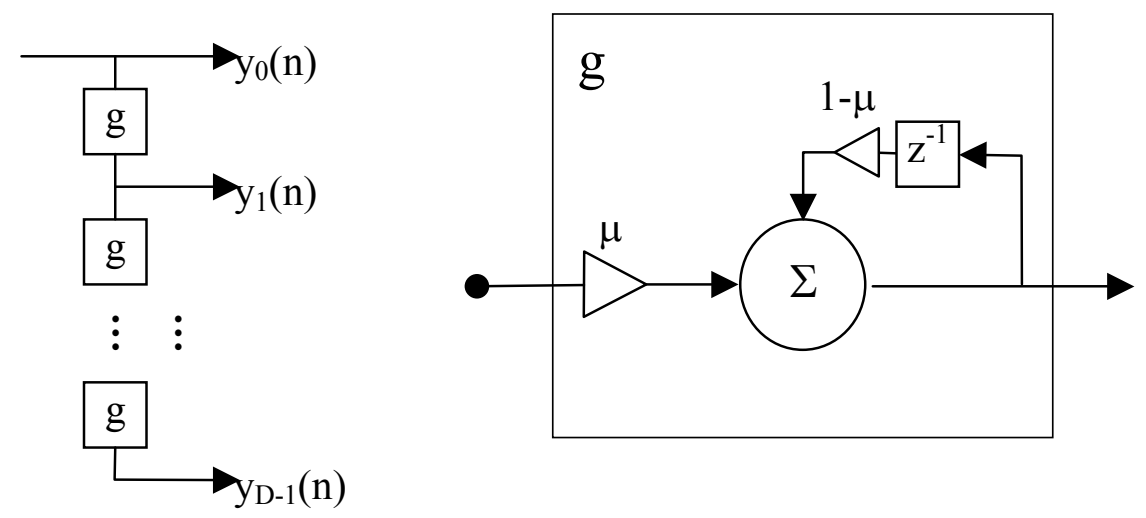

Figure 19 Gamma memory (left) and its recurrent context element (right). 
The delay element's output for the $\mathrm{k}^{\text {th }}$ tap is given by

$$
y_{k}(n)=\mu y_{k-1}(n)+(1-\mu) y_{k}(n-1)
$$

In the z-domain, (B78) can be written as $Y_{k}(z)=\mu Y_{k-1}(z)+z^{-1}(1-\mu) Y_{k}(z)$ or

$$
G(z)=\frac{Y_{k}(z)}{Y_{k-1}(z)}=\frac{\mu z}{z-(1-\mu)}
$$

In the time domain, $y_{k}(n)$ can also be written as $y_{k}(n)=y_{k-1}(n) * g(n)$ where $g(n)=Z^{1}\{G(z)\}$, equal to $Y_{k}(z)=Y_{k-1}(z) G(z)$. Iteratively

$$
Y_{k}(z)=X(z) G^{k-1}(z)(\mathrm{B} 80)
$$

$\mathrm{g}(\mathrm{n})$ has the form of the Gamma function's integrand, and hence comes the name Gamma memory. If the tap outputs of the Gamma memory delay line are fed to a linear neuron for weighed sum, the resulting configuration is called a Gamma Filter.

It should be noted that if $\mu=1$, then the Gamma memory turns into a simple delay line. The feedback portion of the delay element creates an exponentially decaying infinite impulse response (IIR) filter with $h(n)=\mu(1-\mu)^{n-1}$ since $y(n)=\mu x(n)+(1-\mu) y(n-1)$. This theoretically infinite impulse response of the Gamma memory gives it more memory depth (in recall of the past) with a shorter delay line. However, in contrast to the arbitrary impulse response of an FIR, the impulse response of the recurrent IIR has only one control parameter $\mu$.

Time-delay RBF Neural Networks: Besides MLP, one can feed the tap outputs of a memory structure to a RBF neural network. For a simple delay line focused architecture 
such as the one given in figure 17 the overall signal swing will be the same along each time delay axis (tap) since the input signal traverses all the stages in turn, thus the input space will be extensively covered. Since many basis functions such as Gaussian are local (i.e. their magnitudes decrease rapidly as their arguments increase), one may have to use many input bases in order to cover the signal space spanned by tap-delay. The choice of Gamma memory over a simple delay line may help since it has more depth with fewer taps. However, Gamma memories have less resolution because of the low pass averaging action of their recurrent context elements.

Jordan Networks: these networks have a context layer whose outputs go to network's hidden layer. The context layer is made of context memory elements with predefined fixed feedback gain (for instance the Gamma delay kernel shown in Figure 19). The context layer receives its input from the network output. This way, based on the output history (output context) the system can differentiate between incoming temporal patterns (figure 20).

Elman Networks: these networks are similar to Jordan networks with a hidden context layer made of context memory elements with pre-defined fixed (or even adaptive) feedback gains. However, their context layer receives its input from the network's hidden layer (see figure 20). Then based on the on system's internal state history (internal context), the system can differentiate between incoming temporal patterns.

Jordan and Elman networks are capable of producing different results for the same input patterns based on network context layer contents (i.e. different past histories and scenarios). Since the feedback weights are constant, one can use backpropagation during each time step to find the corresponding error-descent weight gradients. The nonadapting feedback weights as well as the general network size and topology leave quite a bit for guessing and trial and error. In Jordan networks erroneous outputs will be fed back to the context layer and may corrupt its contents for future steps. 
Based on the versatile arbitrary configurations that GETnet can assume, Elman and Jordan networks, as well as all memory kernels described above can be realized by it.
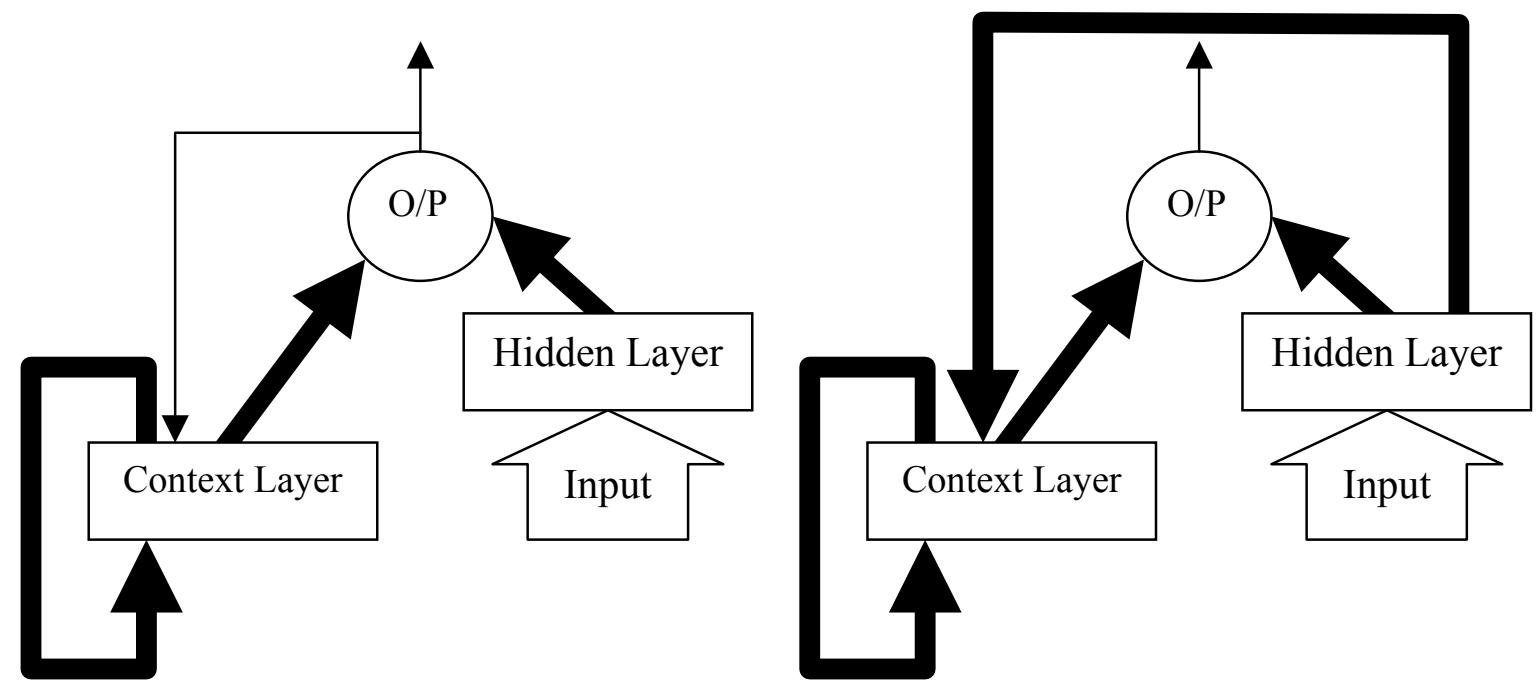

Figure 20 Jordan temporal network (left) vs. Elman temporal network (right). Bold lines represent multiple connections.

\section{General Temporal Neuron Models}

All the studied neural elements studied so far can be categorized as special cases of a Nonlinear Auto-Regressive Moving-Average, or NARMA processing element. A single input, single output causal discrete-time NARMA element of order $(\mathrm{M}, \mathrm{N})$ is defined as

$f\left(x_{1}(n), x_{1}(n-1), \ldots, x_{1}\left(n-M_{1}\right) ; \ldots ; x_{D}(n), x_{D}(n-1), \ldots, x_{D}\left(n-M_{D}\right) ; \ldots ; y(n), y(n-1), \ldots, y(n-N)\right)=0$

The above formula corresponds to a discrete-time system described by a set of difference equations. (B81) can be re-arranged as

$$
y(n)=f_{i o}\left(\ldots, x_{i}(n), x_{i}(n-1), \ldots ; y(n-1), y(n-2), \ldots\right)
$$


and depicted as

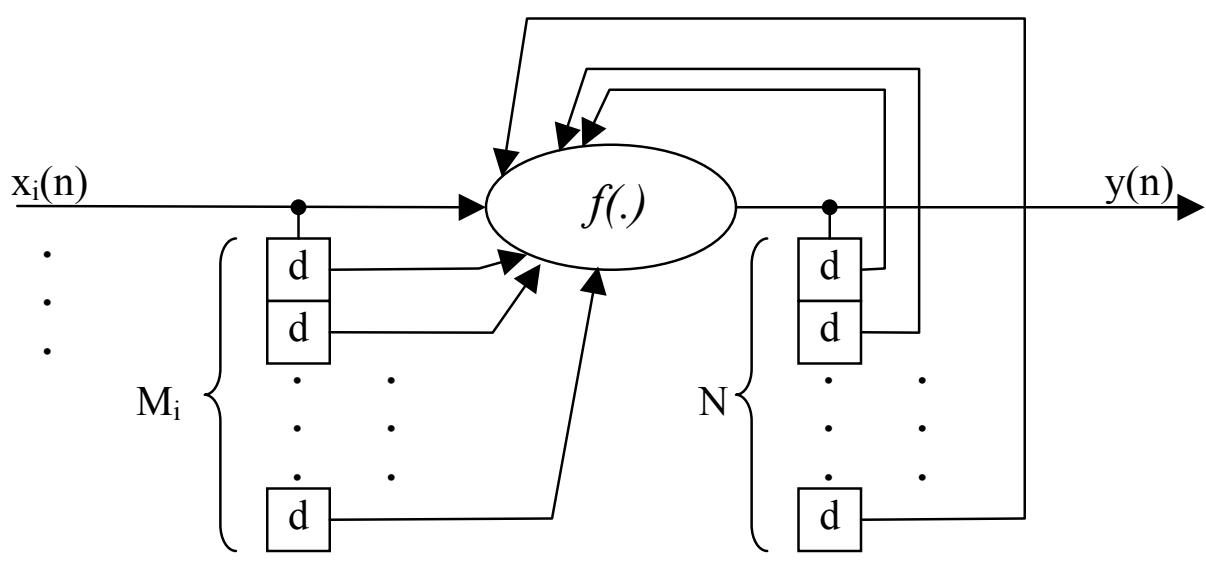

Figure 21 A general nonlinear ARMA element.

In fact the term "moving average" befits a simpler arrangement given later here by (B87) since the nonlinear function $f$ here is not essentially averaging the contents of the sliding time windows captured by delay lines $\mathrm{M}_{\mathrm{i}}$.

Special cases of NARMA include

- $\exists \mathrm{M}_{\mathrm{i}} \neq 0, \mathrm{~N}=0$ (no feedback): (B82) reduces to a nonlinear moving average or NMA described by $y(n)=f_{i o}\left(\ldots x_{i}(n), x_{i}(n-1), \ldots\right)$.

- $\forall \mathrm{x}_{\mathrm{i}}=0, \mathrm{~N} \neq 0$ (no input, just feedback loops): we will have a nonlinear autoregressive element or NAR which displays an output based on its initial conditions described by $y(n)=f(y(n-1), y(n-2), \ldots)$.

- $\forall \mathrm{M}_{\mathrm{i}}=0, \exists \mathrm{x}_{\mathrm{i}} \neq 0$ (no input delay): we will have a nonlinear auto-regressive element with external input (NARX).

NARMA is the most comprehensive model and encompasses the existing ANN and biological models such as Grossberg's additive model and Freeman's model. The continuous version of Grossberg's model is given by 


$$
\frac{d y_{j}(t)}{d t}=-\mu y_{j}(t)+f\left(\sum_{i, i \neq j} w_{j i} y_{i}(t)+b_{j}\right)+I_{j}(t)
$$

$b_{j}$ is the $j^{\text {th }}$ node bias and $I_{j}(t)$ is its external input. Note how this equation resembles that of a leaky integrator (parallel RC circuit) which is indicative of a simple, passive, single compartment biological neural state space model:

$$
\left\{\begin{aligned}
\frac{d v(t)}{d t} & =-\frac{1}{R C} v(t)+\frac{1}{C} \sum_{i=1}^{N} w_{i} x_{i}(t)+\frac{I(t)}{C} \\
y(t) & =f_{\text {sigmoid }}(v(t))
\end{aligned}\right.
$$

with $v(t)$ being the instantaneous membrane voltage, $I(t)$ the external injected current signal, and $w_{i} x_{i}(t)$ the weighed $\mathrm{i}^{\text {th }}$ input current from other node(s). The second equation in (B84) approximates the integrate and fire action of an excitable membrane that converts the internal variable $v(t)$ (membrane voltage) to an instantaneous spiking frequency $y(t)$. Substituting $y(n)$ for $y(t)$ and $y(n)-y(n-1)$ for $d y(t) / d t$ in (B84) yields the discrete time version of Grossberg's model. In general:

$$
y_{j}(n+1)=(1-\mu) y_{j}(n)+f\left(\sum_{i, i \neq j} w_{j i} y_{i}(n)+b_{j}\right)+I_{j}(n)
$$

Variations of this model are the predominant neural models used in artificial neural networks. Note that $\mu=1$ yields the famous McCulloch-Pitts static neural element.

Higher order model such as Freeman's ${ }^{67}$ are used in the modeling of biological systems. Freeman's model represents the rabbit olfactory system and is given as an ensemble of second order neuronal assemblies. The building blocks are defined by the second order differential equation 


$$
\frac{1}{a b}\left[\frac{d^{2} x(t)}{d t^{2}}+(a+b) \frac{d x(t)}{d t}+a b x(t)\right]=Q(x(t))
$$

which represents a second order system in discrete time as well.

In the above models, the nonlinear function $f_{i o}$ in (B82) is responsible for synaptic integration. Activation is considered as a weighted sum of inputs passed through a sigmoidal nonlinearity, as formulated below for a discrete time multiple input, single output causal NARMA node.

$$
y(n)=f_{\text {sigmoid }}\left(\sum_{i=0}^{D} \sum_{m=1}^{M_{i}} w_{i m}^{f w d} x_{i}(n-m)+\sum_{p=1}^{N} w_{p}^{b w d} y(n-p)\right)
$$

Here $\mathrm{D}$ is number of inbound signals ( $\mathrm{x}_{0}=1$ for bias), $w^{f f w}{ }_{i m}$ is the associated weight of the forward connection between the $\mathrm{m}^{\text {th }}$ tap of the $\mathrm{M}_{\mathrm{i}}$ stage input delay line and the $\mathrm{i}^{\text {th }}$ input $\mathrm{X}_{\mathrm{i}} \cdot w_{p}{ }^{b w d}$ is the feedback connection strength of the $\mathrm{p}^{\text {th }}$ tap in the output's $\mathrm{N}$ stage delay line (pre-nonlinearity), and $\mathrm{n}$ is the current discrete time. It can also be shown that the sigmoidal nonlinearity results in limited richer synaptic integrations such as $\Sigma \Pi$ (for instance, the Taylor expansion of the summed inputs $\Sigma$ through the sigmoidal nonlinearity will include all the multiplicative terms $\Pi$.)

\section{Training Recurrent Neural Networks}

For supervised temporal learning, first one should have the desired temporal output, $d_{k}(t)$ for each input signal $x_{k}(t)$, where $k=1,2, \ldots, K$ is the pattern index. Then one can use any norm to calculate instantaneous error and the sum over the period of interest, say $\left[\mathrm{t}_{\mathrm{A}} \mathrm{t}\right]$ for continuous time or $\left[\mathrm{N}_{\mathrm{A}} \mathrm{n}\right]$ for discrete time signals. The corresponding instantaneous $\mathrm{L}_{\mathrm{p}}$ error norm can be written as 


$$
\varepsilon(t)=(y(t)-d(t))^{p}
$$

Replacing $t$ with $n$ will yield the discrete-time version, $\varepsilon(n)=(y(n)-d(n))^{p}$. The resulting aggregated temporal errors will be given by

$$
\begin{aligned}
& J(n)=\sum_{k=1}^{K} \sum_{i=N_{A}}^{n} \varepsilon_{k}(i) \\
& J(t)=\sum_{k=1}^{K} \int_{\tau=t_{A}}^{t} \varepsilon_{k}(\tau) d \tau
\end{aligned}
$$

Two general modes of training can be applied to error signals described in (B88) through (B90): fixed-point or trajectory learning.

- For fixed-point back propagation learning, the input is applied and clamped at each time instance $n$ till the transients of the network are over. Then the corresponding error at that clamped instance is calculated and propagated back through the dual network. Corresponding weight gradients are calculated after the transients of the dual network have died out.

- For trajectory learning, the cumulative temporal error over the period of interest as given by (B89) or (B90) is used. One has to wait for the changes to propagate through all the path delays and show their effects over the whole period of interest (time trajectory) in order to be able to calculate required derivatives.

Static back-propagation cannot be used in adapting recurrent parameters since a change in feedback parameters loops and propagates in time forever. However, the network topology imposes a specific order on system state updates that remains constant. This leads to an ordered list such as the one implied for ordered derivatives in (B47) and enables one to derive networks' variable sensitivities through time. More specifically, 
consider a temporal neural network of $\mathrm{N}$ nodes and their corresponding internal states (instantaneous node activations) $S(n)=\left\{y_{i}(n)\right\}$ and connection weights $W=\left\{w_{j i}\right\}$. The Ntuple $S(n)$ describes a static, non-recurrent network in each time-snapshot $n$ and has an ordered list of the instant feed-forward dependencies of the network. The overall temporal dependency list can be written as

$$
L=\{W, S(0), S(1), \ldots\}=\left\{\ldots, w_{j i}, \ldots, y_{i}(0) \ldots, y_{i}(1), \ldots\right\}
$$

One can also arrange weights of an ordered network in a matrix format $W=\left[w_{j i}\right]$ so that the row index $\mathrm{j}$ designates the destination node and the column index $\mathrm{i}$ designates the source node of the connection weight $w_{j i}$. Since for a feed-forward network connections $j>i$, such a network will have all its upper-triangle elements equal to zero, and vice versa. The nonzero diagonal elements indicate the strength of self-feedback in corresponding nodes. Note that no feedback loop can exist without delay; otherwise unrealistic races will take place. Based on the ordered dependence list of (B91) one can derive ordered derivatives needed for back-propagation. Recalling the earlier definition of ordered derivative in an ordered network from (B47) and taking into account the new extended ordered list of (B91) one can write

$$
\frac{\partial^{\text {ord } J}}{\partial y_{i}(n)}=\frac{\partial^{\text {dir } J}}{\partial y_{i}(n)}+\frac{\partial^{\text {ind }} J}{\partial y_{i}(n)}=\frac{\partial^{\text {dir }} J}{\partial y_{i}(n)}+\sum_{\tau>n} \sum_{j>i} \frac{\partial J^{\text {ord }}}{\partial y_{j}(\tau)} \frac{\partial^{\text {dir }} y_{j}(\tau)}{\partial y_{i}(n)}
$$

The index $\tau>n$ ensures that the temporal order in the list $L$ is preserved, and $j>i$ implements the same ordering imposed by the (spatiotemporal-unrolled) structure. Similarly, if we replace $y$ with $w$ in (B92) and simplify, we will have:

$$
\frac{\partial^{\text {ord }} J}{\partial w_{j i}}=\frac{\partial^{\text {dir }} J}{\partial w_{j i}}+\frac{\partial^{i n d} J}{\partial w_{j i}}=\sum_{n} \sum_{k \geq j} \frac{\partial J^{\text {ord }}}{\partial y_{k}(n)} \frac{\partial^{d i r} y_{k}(n)}{\partial w_{j i}}
$$


Note that the direct derivative of error as described in (B88) through (B90) with respect to weights is zero. In addition, since the weights are before all the states in (B91), the time index should run its full course. $\sum_{k \geq j} \frac{\partial J^{\text {ord }}}{\partial y_{k}(n)} \frac{\partial^{d i r} y_{k}(n)}{\partial w_{j i}}$ is nonzero only for $\mathrm{k}=\mathrm{j}$ because of the second term. Thus (B93) can be further simplified into

$$
\frac{\partial^{\text {ord }} J}{\partial w_{j i}}=\sum_{n} \frac{\partial J^{\text {ord }}}{\partial y_{j}(n)} \frac{\partial^{d i r} y_{j}(n)}{\partial w_{j i}}
$$

Similar to the procedure shown in (B47) through (B61), (B92) and (B94) will yield the required weight gradients for backpropagation in a recurrent temporal ordered network of $\mathrm{N}$ nodes within the given time frame $n \in\left[\begin{array}{ll}1 & n_{f}\end{array}\right]$. Now (B94) can be substituted in (B92) to yield the sought gradient component

$$
\frac{\partial^{\text {ord }} J}{\partial w_{j i}}=\sum_{n=1}^{n_{f}}\left(\frac{\partial^{d i r} J}{\partial y_{j}(n)}+\sum_{\tau>n}^{n_{f}} \sum_{k>j}^{N} \frac{\partial J^{\text {ord }}}{\partial y_{k}(\tau)} \frac{\partial^{d i r} y_{k}(\tau)}{\partial y_{j}(n)}\right) \frac{\partial^{d i r} y_{j}(n)}{\partial w_{j i}}
$$

The main challenges for using the above scheme to train temporal recurrent neural networks include:

1. (B95) becomes rapidly costly for bigger networks and longer time spans (bigger $\mathrm{N}$ and $\mathrm{n}_{\mathrm{f}}$ ).

2. One has to find a method for choosing appropriate delays.

3. Co-adapting of weights and delays makes the performance estimate very noisy, and introduces many local minima.

4. It has been shown that it is hard for backpropagation in time to learn long-term dependencies.

Two other remaining issues are the non-causality of the summations in (B95) which can be solved by deferring the calculations to the end of the time trajectory $n=n_{f}$, 
similar to the batch mode; as well as the infamous problem of finding the optimal topology as discussed earlier in terms of the bias-variance dilemma. Gradient descent learning can actually be applied to network delays ${ }^{68,69}$ but still the other mentioned challenges remain. GETnet tries to circumvent these problems by introducing hybrid training and using partial temporal backpropagation, while finding suitable delay structures through an evolutionary process, as described in section C.

\section{Network Energy, Hopfield and Boltzmann Neural Networks}

A different way of looking at temporal networks is through the concept of network energy. Especially in recurrent networks with saturating nonlinearities, one can consider the transition of the network's $\mathrm{N}$ internal states towards a vertex in the N-cube as the convergence of a dynamic system to an equilibrium state under the given constraints and energy function, also known as computing through attractors. Once the given transient state falls within a basin of attraction, the network will converge to the attractor on the bottom of that basin. This convergence, contingent upon its existence, can be straight forward or through a chaotic path or a limit cycle.

The stability of such networks as well as other neural networks with a defined energy function has been studied using stability analysis methods of control systems theory ${ }^{70,71,72}$. Note that neural networks such as GETnet with saturating activation functions such as the sigmoid always have bounded outputs and are stable in the sense of BIBO (bounded in, bounded out). This is further reinforced by the fact that that the teaching data are bounded themselves. Moreover, saturation of nodes in such networks is seen as a form of computation with attractors and is thus deemed an essential part of their function under certain regimes ${ }^{73}$. Convergence of recurrent networks under the concept of network energy is briefly introduced below for a Hopfield nets.

Hopfield Networks: a symmetric, fully connected recurrent network with a hard limiting bipolar activation function is called a Hopfield Neural Network. The input is fed 
to the network as initial states, and then the network is allowed to run freely with nodes' state transition given by

$$
y_{j}(n+1)=\operatorname{sgn}\left(\sum_{i} w_{j i} y_{i}(n)+b_{j}\right)
$$

This network will settle down when $y_{j}(n+1)=y_{j}(n), \forall j$. If the weight vector is computed from the inner product of input patterns $\vec{W}=E\left(\vec{X} \cdot \vec{X}^{T}\right)$ then the Hopfield network will act as an associative memory and is able to converge to a desired pattern even if it receives a partial or corrupted initial pattern key. One can define an energy function for such network as

$$
H=-\frac{1}{2} \sum_{i} \sum_{j} w_{j i} y_{i} y_{j}
$$

It can be shown that a Hopfield network with symmetric weights (a sufficient condition) and the above energy function is stable in the sense of Lyapunov since the network energy $H$ is non-increasing during the course of node transitions. Based on the initial state which can be considered as the network's input, the system will converge to the nearest minimum on the energy surface defined by $\mathrm{H}$ (i.e. an attractor). This is similar to a solid body moving down towards a resting point under the constraints of a surface in order to minimize its gravitational potential energy.

Hopfield networks (and their variants such as Boltzmann machines) are dynamic and undergo temporal changes. They also utilize the notion of computational energy, similar to potential energy in mechanics, in order to simplify the otherwise complex behavior of the recurrent network and explain their computations in terms of attractors. However, the internal temporal changes of such networks do not represent the temporal contents of the external world data, but rather the networks' internal state changes. The same can be said about self-organizing maps. Moreover, the real world networks receive 
and process the temporal information continually, whereas the Hopfield networks receive their inputs only as an initial condition and then are left running free receiving no more information. These networks also suffer from low memory capacity since the number of stored patterns is only $15 \%$ of number of nodes. The other problem is spurious memories (false energy minima), which results in false recalls. Translating other types of problems for Hopfield networks (e.g. coding the problem into an appropriate energy function to be optimized) is also hard, if not impossible.

It is possible to study stability of other neural networks in the sense of Lyapunov through definition of network energy in similar fashion under different update regimes and network architectures ${ }^{74,75,76}$. 


\section{B4 Evolutionary Methods}

\section{B4-1 A Review of Evolutionary Computing}

Evolutionary computing started mainly through works of Holland, Rechenberg, Schwefel, and Fogel as a general purpose and adaptable problem solver. Recently this field has seen an exponential growth because of its flexibility as well as the availability of powerful and affordable computers. Having roots in the evolutionary processes of nature and specially the neo-Darwinian scheme, this discipline tries to mimic the general process that resulted in creation of intelligent and adaptive living organisms. In what follows the focus is mainly on those evolutionary approaches that are of some interest to this research and thus other topics have intentionally received less attention.

\section{Evolutionary Algorithms (EA), General Concepts}

Here a brief introduction to the Evolutionary Algorithms (EA) $77,78,79,80,81,82,83,84$ is given. EA is an essential part of GETnet since it governs both alterations in contents as well as architecture of the sought neural network solutions.

Consider a general optimization problem of finding a vector of parameters $\boldsymbol{X} \in M$ such that a quality criterion which usually is a real-valued function also known as an objective function $f: M \rightarrow \Re$ is maximized. $X^{*}$ is called a global solution if:

$$
\forall \vec{X} \in M: f(\vec{X}) \leq f\left(\vec{X}^{*}\right)
$$

$\boldsymbol{X}^{*}$ is called a local solution if:

$$
\exists \varepsilon>0, \forall \vec{X} \in M: \rho\left(\vec{X}, \vec{X}^{*}\right)<\varepsilon \Rightarrow f(\vec{X}) \leq f\left(\vec{X}^{*}\right)
$$


where $\rho$ denotes a distance measure. The existence of several such local maxima is called multimodality. There might also exist constraints such that only a subset of $M$ like $F$ is considered feasible. Notice that $f$ and $F$ need not to be mathematically defined, especially for real world problems.

The search space $M$ is composed of variables that represent solutions to be optimized (such as neural network parameters). $M$ can even include the adaptation parameters themselves, such as the standard deviation of mutations in GETnet. $M$ is also called the phenotype space. The phenotypes can be encoded into more abstract objects, for instance binary or real-valued strings called genomes or chromosomes. In this case, the space of these encoded parameters is called genotype space. Evolutionary algorithms have a population of $\mu$, which in each generation produces $\lambda$ offspring through search operators such as mutation and recombination (crossover). Each of these search operators has several variants and operates on those parents selected due to their higher fitness values. Through another fitness-based selection (i.e. performance with respect to environment), applied to the pool of offspring and parents, the next generation is selected. In the real world, natural selection chooses organisms that are more successful in garnering the limited available resources while competing against each other in a finite environment. Those are more likely to survive and propagate their genetic material through reproduction. Reproduction is either asexual (e.g. in bacteria, where genome is only subject to transcription error or mutation) or sexual (e.g. mammals, where genome is subject to a further change through parental information recombination).

\section{Modes of Operation}

If $\mu$ parents produce $\lambda$ offspring, and then from the offspring $\mu$ individuals replace the older generation, we have $\operatorname{EA}(\mu, \lambda)$, also called the comma strategy. Note that if some parents have a higher fitness value they would be replaced by their variant offspring 
anyway. Such mode is non-elitist in the hope of avoiding local optima by not allowing such solutions to stay and propagate in population and tolerating temporary deterioration of solutions by the replacing the whole generation.

If $\mu$ parents produce $\lambda$ offspring that compete with parents, $\mu$ individuals from both sides are selected for the next generation and we have $\operatorname{EA}(\mu+\lambda)$, also called the plus strategy. Depending on the selection scheme, this can be an elitist search since the best of the population can always survive unless a score of better solutions oust it.

An intermediate mode will allow each solution to have a lifespan of $\kappa$ generations, e.g. $\operatorname{EA}(\mu, \kappa, \lambda)$. In this view, one can consider the comma strategy at one extreme with $\kappa=0$ and the plus strategy at the other extreme with $\kappa=\infty$.

Evolutionary algorithm needs diversity to operate. Search operators such as mutation and crossover create this diversity. Initial population is created randomly, either entirely or by mutating one individual $\mu$ times. In pseudo-code one can summarize evolutionary algorithms as follows

$t=0$;

initialize $P(t)$; //random initial population

evaluate $P(t)$;

while not terminate /le.g. fitness goal achieved, timeout, etc.

$P^{\prime}(t)=$ variation $[P(t)]$; //e.g. mutation, crossover

evaluate $\left[P^{\prime}(t)\right] ; \quad \quad$ /le.g. assign fitness

$P(t+1)=$ select_survivors $\left[P^{\prime}(t) \cup Q(t)\right] / /$ Fittest to survive for the next generation $t=t+1$;

endwhile

An EA with a higher $\mu / \lambda$ tends to search more globally and converge slower, while a lower $\mu / \lambda$ does a faster but more local search. Notice that EAs are not purely random, since an offspring is not instantiated independently from its parent(s), and it carries its lineage's search history. EAs can be adapted to a wide range of multi 
parameter combinatorial optimization problems, be they linear or nonlinear. The fitness function does not have to be differentiable either, in contrast with methods such as gradient search.

\section{Selection Methods and Variation}

Some search operators for EA are discussed below. Since most of these approaches share concepts and methods for variation and specially selection, the first part on genetic algorithms describes the shared methods in more detail and for subsequent parts only the differences will be mentioned.

\section{Genetic Algorithms (GA)}

This type of EA was introduced by Holland in 1975. GA uses genetic operators on an encoded genotype, which is then decoded back into a phenotype. GA is widely used for optimization problems. Genotypes that eventually represent individuals are binary strings of fixed length (in contrast to genetic programming GP) composed of the encoded parameters to be optimized. Genotype elements (usually binary bits) are called genes. Specific positions where each gene appears are called alleles.

\section{Representation, Decoding and Encoding}

An encoder function like $h$ is needed to map the phenotype space $M$ into genotype space. For instance, the phenotype space could consist of $n$-dimensional real vectors $\left(M \subseteq \mathfrak{R}^{\prime \prime}\right)$ that are mapped into an $(\mathrm{n} \times \mathrm{b})$ binary strings (chromosomes) through linear scaling of each phenotype (i.e. vector component) to $\left[0,2^{\mathrm{b}}-1\right]$ and then into a b-bit binary number 


$$
h: \mathfrak{R}^{n} \rightarrow\{0,1\}^{(n \times b)}
$$

This binary chromosome then will be subject to search operators such as mutation and crossover. The resulting new generation of chromosomes should be mapped back to phenotype space for fitness assessment, etc., through the inverse function $h^{-1}$ :

$$
h^{-1}:\{0,1\}^{(n \times b)} \rightarrow \Re^{n}
$$

The objective function then can assess the fitness of the solution represented by the phenotype

$$
f_{R}: \Re^{n} \rightarrow \Re
$$

or equivalently from the binary chromosome

$$
f:\{0,1\}^{n \times b} \rightarrow \Re^{n}
$$

where $f=f_{R}$ o $h^{-1}$

Note that the genetic encoding and decoding functions $\mathrm{h}$ and $\mathrm{h}^{-1}$ and the more complex objective function $f=f_{R} \mathrm{o} h^{-1}$ may introduce more complexity and even multimodality compared to the original $f_{R}$. GETnet uses direct mapping and phenotype evaluation based on $f_{R}$.

The choice of binary representation has been justified by interpreting GAs' behavior in light of schema theory, where it is assumed that detrimental effects of mutation and crossover lead to survival of the shortest and lowest-order schemas that are supposed to be the data building blocks of the evolving solutions. However, this analogy is not very strong. Moreover, for some theoretical and many practical problems such as 
GETnet, real valued representations are more suitable than binary. Real-valued genotypes are discussed later under evolution strategies (ES).

\section{Parent Selection}

In GA, one needs to know whether all the computing resources for the next generation should be devoted to individuals currently occupying a promising neighborhood (strict parent selection) or rather dispersed randomly across the entire search space. These two extremes depict the trade-off between degenerating to a local search on one hand and a very slow global search on the other hand. Usually an inbetween compromise is made. The parent selection policies can be classified as follows:

Dynamic vs. Static: In static methods, selection is made based on the fitness of the whole population, while in the dynamic method the selection is based on local tournaments.

Preservative vs. Extinctive: In preservative methods, each individual is guaranteed to receive a reproduction probability bigger than zero, e.g. linear ordering with $\beta<2$ or roulette wheel selection as described later here. However, in extinctive selection methods, some individuals may not be given a chance for reproduction, e.g. linear ordering with $\beta \geq 2$.

Elitist vs. Purist: Elitist methods guarantee selection of the fittest member, while in the purist version this does not hold. It has been shown that the canonic purist GAs will never converge to a global optimum regardless of initialization, crossover operator and objective function ${ }^{85}$. 
Selection Techniques: some more popular schemes are described below.

Relative Fitness Selection: This technique is also known as stochastic sampling with replacement or a roulette-wheel based selection scheme. In this technique, the relative chance of an individual such as $\boldsymbol{X}_{i}$ for reproduction is given by:

$$
P_{\text {parent }}\left(\vec{X}_{i}\right)=\frac{f\left(\overrightarrow{X_{i}}\right)}{\sum_{j=1}^{\mu} f\left(\overrightarrow{X_{j}}\right)}
$$

Positive fitness values are needed for the above selection. Roulette wheel selection needs a selection fitness function $f$. There might be the problem of a super individual, i.e. an individual with very high relative fitness. This individual will parent most of the next generation and the search may prematurely converge to a local optimum. However, roulette wheel selection is preservative and may help faster convergence by giving relatively better solutions more reproduction chance.

Linear Ordering: One can assign the reproduction probability linearly to the $\mathrm{N}$ individuals according to their sorted fitness

$$
P(i)=\operatorname{MAX}\left\{\frac{1}{\beta}\left(\beta-2(\beta-1) \frac{i-1}{\mu-1}\right), 0\right\} \quad \beta \geq 1, \quad i=1,2, \ldots \mu
$$

$\beta$ controls the selection pressure. If $\beta=1$, then $P(i)=1$ for all $i$, and thus this method degenerates into a uniform random sampling. For $1<\beta<2$ the method is preservative, and for $\beta \geq 2$ it becomes extinctive (see figure 22). A variation of this method called exponential ordering is also used. 

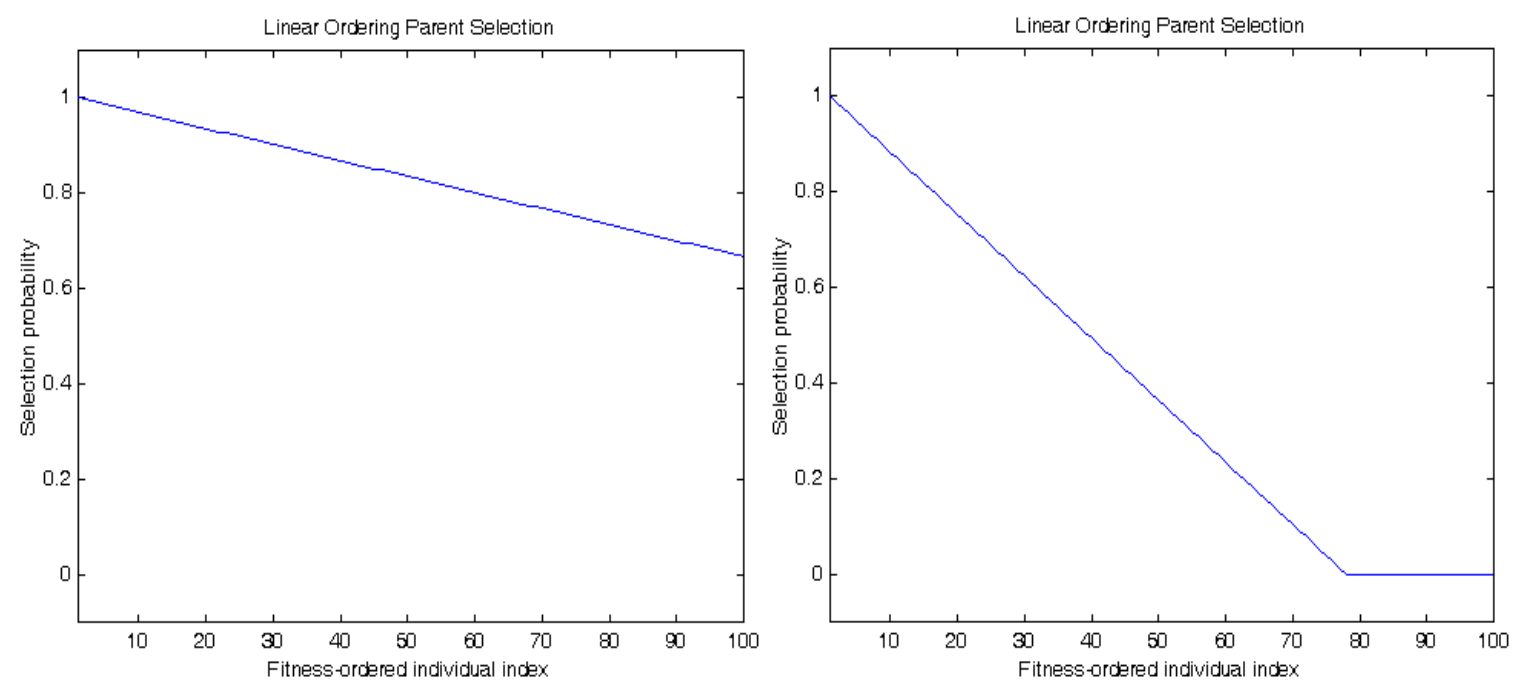

Figure 22 Linear ordering selection probability for a population of $\mu=100$ and $\beta=1.2$ (left), and $\mu=100, \beta=2.8$ (right).

Tournament Selection: In this method, T individuals from the parent generation (with $\mathrm{T}$ being the tournament size) are randomly drawn $\lambda$ times. The best of each subgroup is the one that scores the most wins (one out of T, $\lambda$ times). A bigger tournament size increases selection pressure.

Truncation: This method was invented and used in the earlier works in the field. Simply a fitness threshold is chosen, and the individuals with fitness values above this threshold are selected for reproduction.

GAs usually use $(\mu, \lambda)$ mode. That is, the new population replaces the old one.

\section{Search Operators}

Mutation: This is an asexual search operator resembling genetic transcription error. It is used to avoid stagnation in an evolving population and for introduction of new solutions. Individuals are selected for mutation with a low probability $p_{m}$. Then alleles are 
randomly chosen and their bits are negated. In binary encoded parameters the bit inversion might act upon any bit of the parameter regardless of its significance, thus sometimes the Gray code is used. Then the Hamming distance of 1 resulting from such a single bit mutation will result in single increment in binary parameter value, regardless of mutated bit position. One can also start with larger $p_{m}$ and decrease it over the course of the evolution as it reaches an optimum, similar to simulated annealing. Both the smooth mutations and their extent are controlled through novel real methods in GETnet. Selfadaptation of GA mutation has been suggested by incorporation of $p_{m}$ as a binary number into the chromosome and thus subjecting it to evolutionary optimization. Self-adaptation will be described later in Evolutionary Strategies (ES), since it is one of main features of ES.

Crossover: This is a sexual search operator resembling genotype mixing in multiparent reproduction. It reveals dominant or non-dominant phenotypes or intra-species variations. Individuals are selected with a probability $p_{c}$ for recombination. The selected parents will have parts of their chromosomes marked by random pointers and swapped. Self-adaptation of crossover in GA has been suggested by including the number and position of crossover points into the chromosome, so they would adapt automatically to the problem at hand (called punctuated crossover). A simpler method includes a bit in the chromosome that chooses between simple and two-point crossover. Crossover is not used in GETnet since in practice it destroys the distributed knowledge spread throughout a neural network.

\section{Evolutionary Programming (EP)}

Mainly introduced by L.J. Fogel in 1960s as an approach to artificial intelligence, this is another optimization technique very close to evolution strategies (ES). However, EP was developed independently, initially to evolve finite state machines (FSM) that 
could learn and predict a sequence of symbols. The mechanisms that are different from GA are described briefly below.

\section{Search Operators}

Mutation: In original EP mutations were discrete (FSM state transitions, their number, changing the initial state). When real valued parameters are used, EP becomes very similar to ES. Instead of emphasizing on imitating the complex genetic encoding as it happens in nature (i.e. genotypical evolution), EP focuses on behavioral evolution of a population on the species-level (i.e. phenotypical evolution). Thus, the solutions can directly represent the problem parameters, usually real vectors $M \subseteq \Re^{n}$. For instance, parameters can directly represent weights of a neural network, perturbed by zero mean Gaussian mutations then and connections can also be randomly added or deleted, as is the case in GETnet. The perturbations are supposed to be in a way that small ones are more likely to happen so a strong behavioral linkage between offspring and parents is maintained while the macroevolution caused by mega-mutations (as seen in punctuated equilibrium) is not ruled out. As in GA, it is assumed that there is an optimal solution and that solutions can be coded into a set of variables. Fitness of a solution is calculated from its objective function values. EP uses a range of mutation operators on the current generation to produce competing offspring. Composition of the next generation is based on the fitness, usually through randomly drawn tournaments. No crossover is used since EP is supposed to be at the species level and not individual. Population size might vary as well. EP also subjects the mutation parameters to evolution by bundling individual solutions with search parameters (e.g. variance of Gaussian mutations). These parameters themselves will be subjected to adaptation through perturbation and eventually selection, based on the quality of the offspring for which they are responsible.

\section{Selection}

Probabilistic methods described in the GA selection section apply to EP as well. 


\section{Evolution Strategies (ES)}

ES was introduced by a group of German researchers, most notably Hans-Paul Schwefel. Besides a deterministic selection and utilization of crossover, ES is identical to EP. An ES solution includes a vector of object variables $\boldsymbol{X}_{i}$ and a vector of strategy variables $\sigma_{i}$. Adaptation of strategy variables makes the solutions learn the fitness landscape of the given problem.

Mutation: mutation takes place in the form of additive, zero mean Gaussian perturbations $\sigma_{\mathrm{i}}$ applied afresh and individually to each element in a solution $\boldsymbol{X}=\left(x_{1}, x_{2}, \ldots x_{n}\right)$

$$
x_{i}{ }^{\prime}=x_{i}+\sigma_{i} N_{i}(0,1)
$$

Self-Adaptation: adjustment of the step size $\sigma_{\mathrm{i}}$ is a non-trivial problem. In ES, problems of this type are solved by bundling the strategy parameters with the solution and letting them adapt together. Thus an individual will consist of the solution plus the mutation strategy parameter $\sigma=\left(\sigma_{1}, \sigma_{2}, \ldots \sigma_{n}\right)$

$$
\boldsymbol{a}=(\boldsymbol{X}, \sigma)
$$

where $\sigma \in \mathfrak{R}_{+}{ }^{\mathrm{n}}$ and usually $\mathbf{X} \in \mathfrak{R}^{\mathrm{n}}$. For strategy parameter $\sigma$ Schwefel $^{86}$ suggested that

$$
\begin{aligned}
& \sigma_{i}{ }^{\prime}=\sigma_{I} \exp \left[\tau \mathcal{N}(0,1)+\tau N_{i}(0,1)\right] \\
& \tau^{\prime} \propto 2^{-0.5} n^{-0.5}, \tau \propto 2^{-0.5} n^{-0.25}
\end{aligned}
$$

The older form of self adaptation is additive:

$$
\sigma_{i}^{\prime}=\sigma_{i}\left[1+\alpha N_{i}(0,1)\right]
$$


The additive adaptation can be thought of as the first order Taylor series approximation of (B108). It has been shown that (B109) actually performs similar to (B108) for small $\alpha$ and $\tau$. The linear model might also perform better with noisy objective functions.

Note that the first part of a parameter variance $\tau \mathcal{N}(0,1)$ is the same for all parameters in the solution but the second part $\tau N_{i}(0,1)$ is initialized and applied individually to each $\mathrm{x}_{\mathrm{i}} \in \mathbf{X}$. Existence of a separate, independent $\sigma_{i}$ for every dimension of the search space means that the perturbation will be within a hyper-ellipsoid. This is the evolution method used in GETnet, and in section C these principles are demonstrated through simulations. If all $\sigma_{i}$ are equal, the search neighborhood will reduce to a hypersphere. More elaborate schemes include correlated $\sigma_{i}$ that will allow rotations of the mutation hyper-ellipsoids in the search space. All these degrees of freedom will lead to a better adaptation of evolution to the given problem's fitness landscape, but at the expense of increased computational time complexity.

The order in application of mutation is very important. The strategy parameter vector $\sigma$ should be mutated before being used at each step, since an offspring with a good object vector $\mathbf{X}$ but a poor strategy vector $\sigma$ might be created otherwise. That is, the individual mutated towards a worse situation while its $\sigma$ has no role in its current placement.

Crossover: in ES, unlike GA, recombination is performed on either the whole population or none. It can take the form of simple swapping or linear combination of two or more parents over the whole population $\mu, \lambda$ times, as well as other forms of averaging such as geometric averages. Deciding on the type of ES crossover depends on the problem, objective function, search space dimension, and the number of strategy parameters. Usually the recombination type for object variables differs from that of strategy variables. The number of parents is usually either 2 or $\mu$. ES and EP both usually 
act directly on real valued solutions from $M \subseteq \Re^{n}$ to solve combinatorial optimization problems, without the genetic mapping of GA. Other variants such as mixed integer and real parameters are also possible. ES and EP both include self-adaptation of search operators' parameters. EP selects those solutions receiving the most wins against others in randomly drawn competitions but ES uses deterministic selection based on relative fitness values.

Selection: deterministic versions of the methods described in GA selection are used for the ES selection processes.

There are various other evolutionary algorithm spin-offs such as Genetic Programming (GP), where the evolving solutions are not fixed length strings of parameters but actual computer programs (series of instruction) that provide solutions to a problem. These forms of EA are out of the scope of this research and will not be addressed here. 


\section{B4-2 Application of Evolutionary Methods to Artificial Neural Networks}

Evolution can be used to evolve neural networks' connection weights, architecture, and even learning rules. Methods described below use the performance (defined as the inverse of an error norm) of each network in a population as well as other parameters such as regularization metrics as measures for fitness of a neural network. These methods are classified according to their encoding, and the focus is mainly on the evolution of architecture. Regular gradient descent techniques can be used to measure quality of classification and thus the fitness score of each resulting network ${ }^{87,88,89}$.

\section{Direct Method}

In this encoding, neurons are ordered and labeled from 1 to $\mathrm{N}$ and a binary $\mathrm{NxN}$ matrix (i.e. network's directed graph matrix) is formed as follows: existence of a 1 in row $\mathrm{i}$, column $\mathrm{j}$ implies that neuron $\mathrm{i}$ is receiving an input from neuron $\mathrm{j}$. If the upper triangle is forced to zero, then the network will be feed-forward. The diagonal elements of the matrix can be interpreted as presence of self-feedback on the corresponding neuron. GA can be applied to a population of such individuals as described earlier. If one uses the connection weights in the above matrix, application of ES can evolve the weights at the same time. A variation of this encoding method is used in GETnet.

\section{Graph-Generating Grammar}

In biological systems, there is no direct mapping from genotype to phenotype; instead production processes are responsible for development of the individuals, also called ontogenesis. The Lindmayer system (L-system), which was originally used for the 
simulation of plant development, is an example of such a production processes that has been applied to evolutionary neural network construction. Grammar rules such as $S \rightarrow a S b$ are applied repeatedly until a string for description of the network is produced. $S, a$, and $b$ are called the generation grammar alphabets. Graph generation grammar is such an Lsystem used for creation and evolution of neural network architecture.

The evaluation of genotypes based on the phenotype as well as enforcing tight behavioral links between generations is not very easy with such methods. Furthermore application of this method to evolving networks with distributed memory structures is not straightforward.

\section{Cell Space Method}

This method also was inspired by observations from the development of biological systems' central nervous systems, where each neuron occupies a specific spatial location and then grows its axonal and dendritic trees to make connections to the other neurons in its vicinity. In this scheme, each chromosome is divided into subsections that define a neuron as follows:

$\mid$ Neuron type (input, output...) $\mid$ bias $\mid$ weights $\mid$ segment length $\mid$ branching angle $|x| y \mid$

So each neuron knows where to go $(\mathrm{x}, \mathrm{y})$, how to form its dendritic tree (branching angle, segment length), and what weights and biases assign to them. One of such networks by Nolfi and Parisi is depicted in figure 23.

Another method derived from cell space is generative cell space encoding that includes cell division and migration as well to allow the neuronal population to grow. 


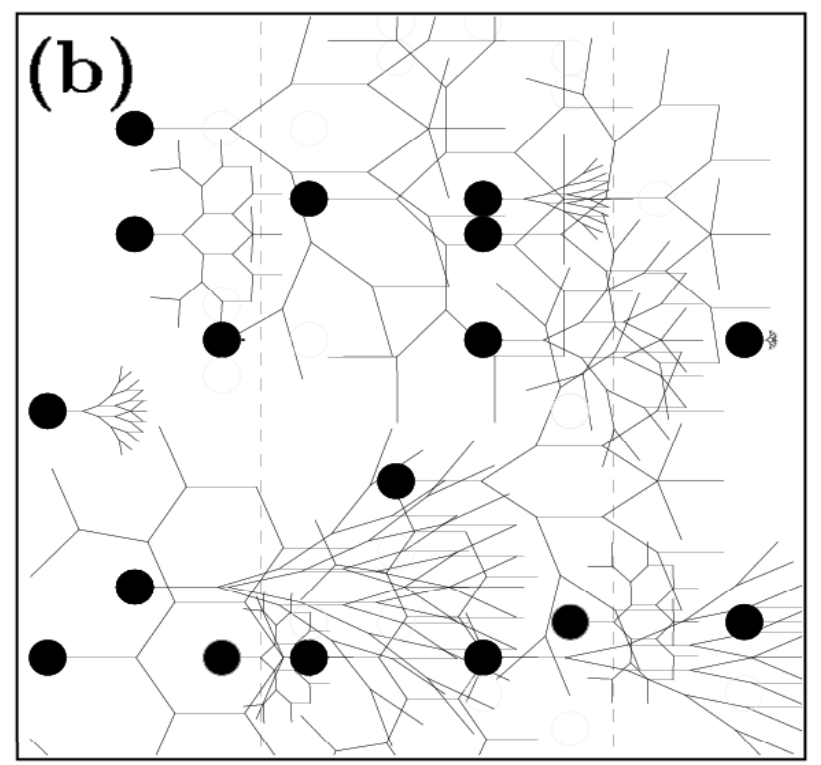

Figure 23 A network resulted from Nolfi and Parisi cell spacing encoding. ${ }^{90}$

Based on the same reasons described for graph generating methods, this method was not chosen for GETnet.

\section{Co-Evolution of Architecture and Parameters}

In addition to described methods, another suggested variation of a direct mapped method that adds training error evaluation is $\mathrm{EPNet}^{91}$. It does not use crossover, because as for many other evolutionary neural network methods, crossover usually has a destructive effect on the system. This should not come as a surprise since the basic idea behind neural networks is distributed representation, while crossover swaps localized blocks and thus will destroy the distributed contents.

In EPNet, first a random population is trained briefly. Then in an evolution loop, after partial training of the current generation, four mutations are applied to the network: neuron deletion, connection deletion, connection addition, and neuron addition. These mutations are applied sequentially and in turn, one at a time. At any point, if a mutation 
results in better performance, no further mutations are applied to that net. This, considering the order of mutations, encourages evolution of more compact neural nets. A flowchart for EPNet is given below.
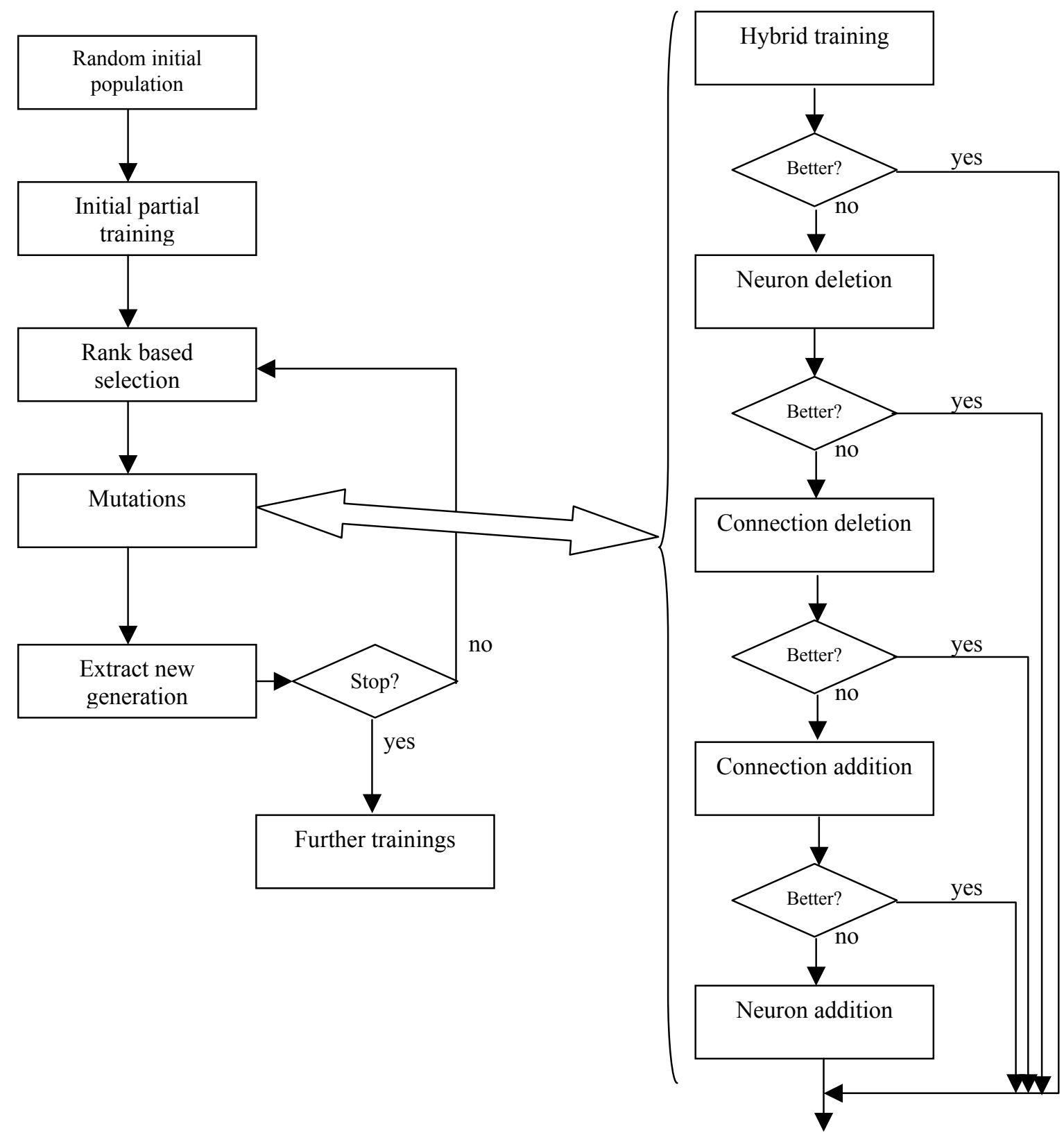

Figure 24 EPNet. 


\section{C: SUGGESTED GENERAL EVOLUTIONARY TEMPORAL NEURAL NETWORK GETnet}

\section{C1 Introduction}

As described earlier, one of the main hindrances of using existing intelligent systems especially neural networks has been the need for extensive human expert involvement for customizing each network to the given task. This issue becomes worse when even the experts do not readily know what type of network arrangement to use, which is usually the case for temporal data and sequence analysis. Even in the event of a rather good guess for topology of a network, there are no analytical methods to ensure the quality of the chosen formation. The author has developed a framework for a general evolutionary temporal neural network, or GETnet, as one approach to address the mentioned issues. The naming convention follows Yao's EPnet ${ }^{91}$, a term he coined for his evolutionary programming networks discussed earlier during the background section B4-2. GETnet utilizes a combination of Lamarckian and Darwinian evolutions and existing training rules to guide each generation of temporal neural networks towards their predefined goals under hybrid supervised training. The evolutionary component also makes GETnet adaptive, since a changing environment reflects its dynamism in training and evaluation data and thus will steer the hybrid training and the evolutionary design accordingly. This is especially true for the evolutionary strategies (ES) method used for GETnet, since the strategy parameters themselves are a part of the evolution and thus adaptable.

GETnet finds the topology, size, connection sparsity, distributed memory depth and structure, synaptic connection strengths, and description complexity of the answer through a hybrid system of deterministic and stochastic searches in weight, delay, and architecture spaces. GETnet can evolve a general class nonlinear recurrent neural networks $(\mathrm{RNN})$ with distributed delay structures. RNNs can represent arbitrary dynamic 
systems ${ }^{19,20}$ and are at least as powerful as Turing machines ${ }^{21}$. However, learning longterm dependencies with gradient descent becomes difficult because of vanishing gradients or forgetting behavior ${ }^{92,93}$, since for information latching the Jacobian of a network's internal states exponentially approaches zero with back-propagation through time (BPTT). Alternative methods have been suggested ${ }^{94,95,96}$, but each has its own deficiencies. For instance, one can feed the network global features or boost the information from far past, but the network may miss short-term dependencies or not converge, also adding to the baby-sitting problem of the network. The addition of evolutionary search component in GETnet is an attempt to overcome the mentioned problem as well as an escape mechanism from local minima.

Recalling from the introduction and according to the flowchart of figure 25, GETnet's algorithm starts with importing the teacher data, and then it generates the initial population of temporal neural networks randomly, with each neuron connected either to itself or to other neurons with single or multiple branches (feed forward, recurrent, or both). Each branch has its own weight and delay. This population then enters the main evolution loop. The termination condition is either reaching desired precision or a maximum time. The evolution adapts number of branches, connections, and nodes as well as other network and strategy variables. Structural mutations try to be non-disruptive to reduce the noise in evolutionary assessment of parameters and avoid obvious deadends. The fitness of each individual is calculated as the inverse of its MSE. Partial gradient descent training is performed before each evaluation. The training time is limited to favor more compact networks over bulky and sluggish networks and achieve a temporal MDL. Pruning also reduces the size of the evolving networks, resulting in minimum model variance and thus better generalization. The weight contents are inherited by the mutant offspring. This transfer is perturbed by an evolving controlled noise to allow room for "individual ingenuity". After the evolution phase, the last generation is fully trained and the best and committee of networks' outputs are computed. 
During the following sections, first the structure of a network in GETnet as an individual with a direct-mapped genotype will be explained. Then a description of each participating module and its function, as depicted in figure 25 , will be given. Simulation results are presented at the end, and a final discussion concludes this last section of this dissertation. 


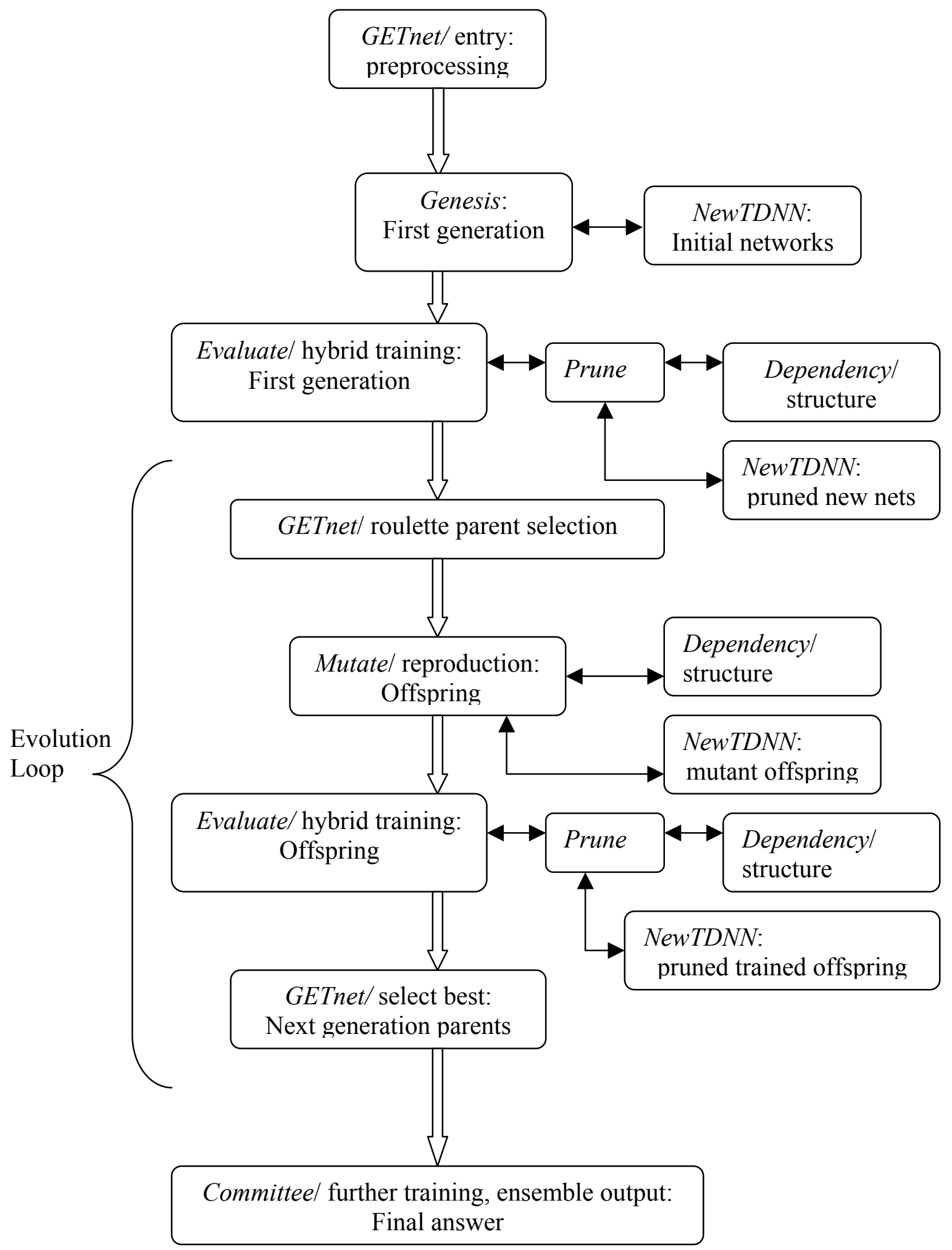

Figure 25 GETnet's flow and organization. The names of actual main modules are italicized, and product of each stage appears after the colon. Secondary helper modules Stat and StatN are not shown for simplicity. 


\section{C2 Description of the Algorithm}

Note: the terms node and neuron are used interchangeably, both denoting a modified Mcculloch-Pitts neuron with summing inputs, sigmoidal activation function, multiple weighted delayed inputs, and delayed self feedbacks.

\section{Network Structure}

GETnet is written with Matlab version 6.5 and its neural network toolbox version 4. It uses a modified formalism for network object description based on Matlab's neural network toolbox. The following is a short description of the direct-mapped genotype contents defining each network. The genotype parameters form weight, delay, structure, and strategy search spaces that drive participating networks towards the desired phenotypical goal.

To illustrate the network's genetic representation, consider the following example:

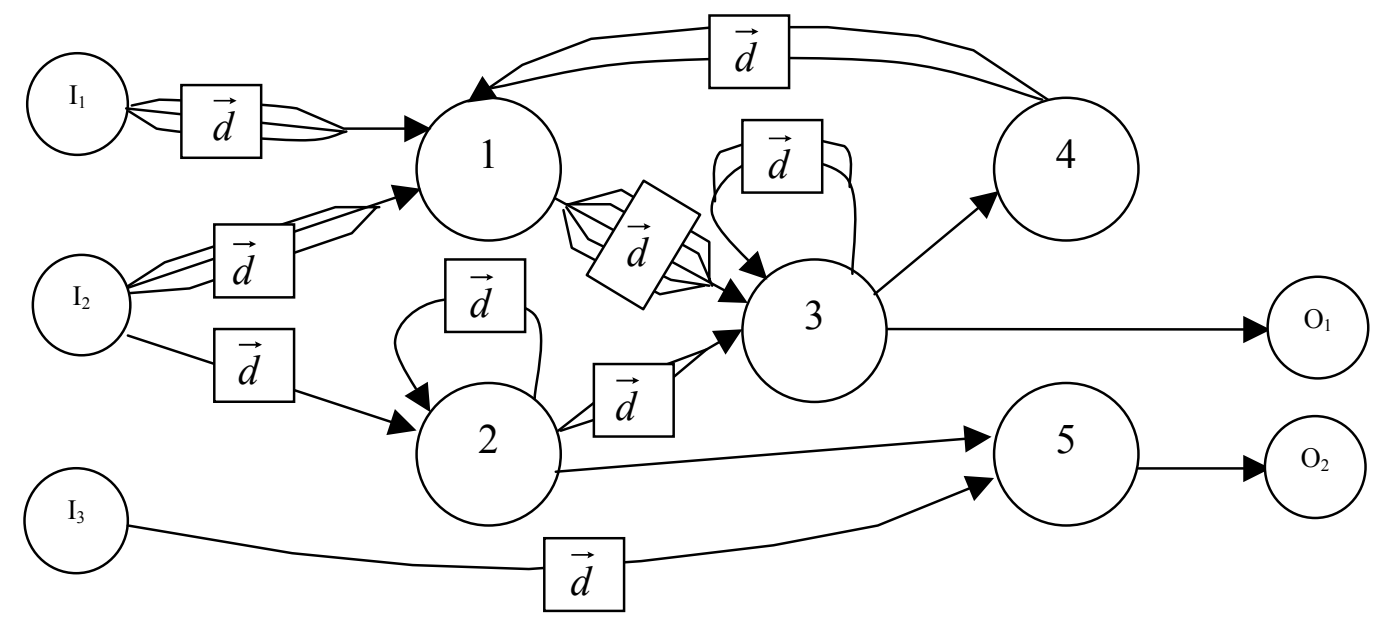

Figure 26 A sample network such as the ones generated by the Genesis module. 
A network is described by its direct genetic encoding under the following four general categories: (1) connection maps, (2) connection branch weight matrices, (3) connection branch delay matrices, and (4) Darwinian input and layer standard deviation matrices, described each in the following section.

1-Connection maps input_connect, layer_connect, and output_connect:

Connection map between inputs and nodes (input_connect): This is a binary matrix with each column referring to an external input, and rows indicating network nodes (hidden and output). That is, input_connect $(\mathrm{r}, \mathrm{c})=1$ indicates that the external input $\mathrm{c}$ is fed to network node $\mathrm{r}$. Since the flow of signals is unidirectional, this type of matrix represents the directed graph (digraph) of the network's input-to-node communications. The columns indicate inputs while the rows indicate nodes, 1 for connection and 0 for no connection between input and nodes. These connections can be with no delay or through a series of parallel delays (connection branches), as described by input_delay and input_weight structures, described in the next sections. For instance, consider the network depicted in figure 26 with 5 neurons, 3 external inputs, and 2 nodes designated as output. The first input $I_{1}$ is connected to the first node, the second input $I_{2}$ connected to the first and second nodes, and the third input connected to the fifth node. Then the input_connect matrix will be as follows:

$$
\text { input_connect }=\left[\begin{array}{lll}
1 & 1 & 0 \\
0 & 1 & 0 \\
0 & 0 & 0 \\
0 & 0 & 0 \\
0 & 0 & 1
\end{array}\right]
$$

Connection map between nodes (layer_connect): This binary matrix represents a digraph similar to input_connect. However, it describes the node-to-node and each node's self feedback connections. Columns correspond to the source nodes and rows correspond 
to the destination nodes. That is, layer_connect $(\mathrm{r}, \mathrm{c})=1$ indicates that the output from node $\mathrm{c}$ is connected to the input from node $\mathrm{r}$. These connections can be with no delay or through series of parallel delays (connection branches), as described by layer_delay and layer_weight structures, described in the next sections. Based on what was discussed above, the layer_connect matrix of the network depicted in figure 26 will be as follows

$$
\text { layer_connect }=\left[\begin{array}{ccccc}
0 & 0 & 0 & 1 & 0 \\
0 & 1 & 0 & 0 & 0 \\
1 & 1 & 1 & 0 & 0 \\
0 & 0 & 1 & 0 & 0 \\
0 & 1 & 0 & 0 & 0
\end{array}\right]
$$

The numbering scheme used for GETnet nodes assumes that they have a sequential order, indexed in an ascending manner from left to right. That is, a network of $\mathrm{N}$ neurons, in terms of its constituting nodes, can be by described by an ordered list:

$$
\text { net }=\left\{n_{1}, n_{2}, \ldots, n_{c}, \ldots, n_{r}, \ldots, n_{N}\right\}
$$

Note that this spatial scheme does not impose any constraints on an arbitrary desired network topology. More formally, the mentioned indexing scheme is used for creation of the network connection digraph defined as a binary relation on the set of $\mathrm{N}$ indexed nodes, and the entire $\mathrm{N}$ ! possible different connection matrices represent isomorphic digraphs.

Consider a feed-forward network in a layered arrangement with one node per layer and left to right indexing. The nonzero elements of such network's layer_connect matrix of have source node indices that are less than those of the destination nodes

$$
\forall n_{r}, n_{c} \in \text { net }, r>c \leftrightarrow \text { layer_connect }(r, c) \in\{0,1\}, r<c \leftrightarrow \text { layer_connect }(r, c)=0
$$


This means that for a feed forward network, the upper triangle and main diagonal elements of layer_connect are zero. The upper triangle and main diagonal elements (row $\geq$ column) of a recurrent network will have nonzero elements. Nonzero elements on the main diagonal (layer_connect $(\mathrm{i}, \mathrm{i})=1)$ indicate self-feedback for node $\mathrm{i}$ (digraph loops). Such feedback loops, as mentioned during the background section, are the basis of longer-term memory kernels such as the Gamma memory. Creating a minor zero triangle (with each side having $n_{o}$ zeros) on bottom right of layer_connect will remove lateral connections for the last $n_{o}$ neurons. This is used in Genesis for feed-forward option to start from a traditional output layer with no lateral connections, which is subject to change by mutation later on. Other lateral connections can act as decorrelators according to (B25).

Output connection map (output_connect): This binary vector designates nodes whose outputs will serve as the network output. If $n_{i}$ is an output node, then the $i^{\text {th }}$ component of output_connect is 1, 0 otherwise. For our example in figure 26 output_connect will be given by:

$$
\text { output_connect }=\left[\begin{array}{l}
0 \\
0 \\
1 \\
0 \\
1
\end{array}\right]
$$

2- Connection branch weight matrices input_weight and layer_weight:

Weights between inputs and nodes (input_weight): This is a matrix with the same dimensions as input_connect. Its elements are null vectors when the corresponding element in input_connect is zero i.e. input_weight $(\mathrm{r}, \mathrm{c})=[]$ iff input_connect $(\mathrm{r}, \mathrm{c})=0$, and a vector of weight values for each connection branch otherwise, i.e. input_weight $(\mathrm{r}, \mathrm{c})=\left[\mathrm{w}^{1}{ }_{\mathrm{i}}\right.$ 
$\mathrm{r}, \mathrm{c}^{2} \mathrm{~W}_{\mathrm{ir}, \mathrm{c}} \ldots \mathrm{w}_{\mathrm{i} r, \mathrm{c}}^{\mathrm{D}}$ iff input_connect $(\mathrm{r}, \mathrm{c})=1 . \mathrm{w}_{\mathrm{ir}, \mathrm{c}}^{\mathrm{j}}$ indicates the $\mathrm{j}^{\text {th }}$ branch weight for the connection between input $\mathrm{c}$ and destination node $\mathrm{r}$. $i$ indicates that these weights come from inputs. For the network in figure 26 we have

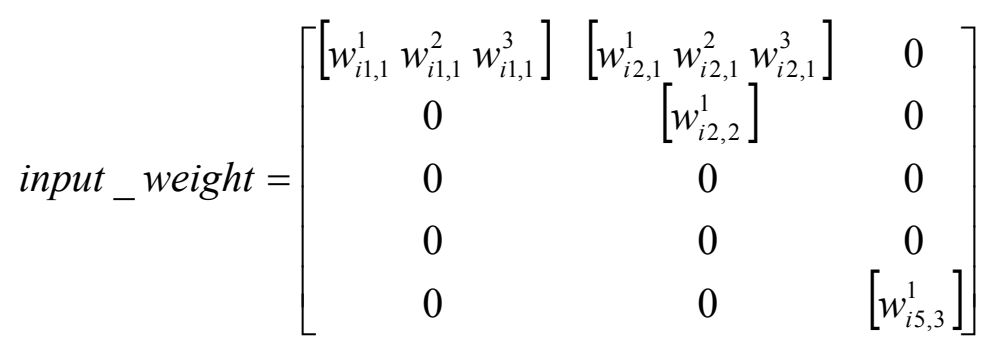

Weights between nodes (layer_weigh)t: this is a matrix similar to input_weight and with the same dimensions as layer_connect. Its elements are null vectors when the corresponding element in input_connect is equal to zero, i.e. layer_weight $(\mathrm{r}, \mathrm{c})=[]$ iff layer_connect $(\mathrm{r}, \mathrm{c})=0$, and a array of weight values for each connection branch otherwise i.e. layer_connect $(\mathrm{r}, \mathrm{c})=\left[\mathrm{w}^{1}{ }_{1 \mathrm{r}, \mathrm{c}} \mathrm{w}_{1 \mathrm{r}, \mathrm{c}}^{2} \ldots \mathrm{w}_{1 \mathrm{r}, \mathrm{c}}^{\mathrm{D}}\right]$ iff layer_connect $(\mathrm{r}, \mathrm{c})=1 . \mathrm{w}_{1 \mathrm{r}, \mathrm{c}}^{\mathrm{j}}$ indicates the $\mathrm{j}^{\text {th }}$ parallel branch weight for the connection between the source node $\mathrm{c}$ and destination node $\mathrm{r}$. $l$ indicates that these weights run between nodes. These parallel, scaled, and delayed copies of a traveling signal constitute the FIR (Finite Impulse Response) action of the feed forward paths and the IIR (Infinite Impulse Response) properties of the feedback loops of GETnet. For the network in figure 26 we have

$$
\text { layer_weight }=\left[\begin{array}{ccccc}
{[]} & {[]} & {[]} & {\left[w_{l 1,4}^{1} w_{l 1,4}^{2}\right]} & {[]} \\
{[]} & {\left[w_{l 2,2}^{1}\right]} & {[]} & {[]} & {[]} \\
{\left[w_{l 3,1}^{1} w_{l 3,1}^{2} w_{l 3,1}^{3} w_{l 3,1}^{4} w_{l 3,1}^{5}\right]} & {\left[w_{l 3,2}^{1} w_{l 3,2}^{2}\right]} & {\left[w_{l 3,3}^{1} w_{l 3,3}^{2}\right]} & {[]} & {[]} \\
{[]} & {[]} & {\left[w_{l 4,3}^{1}\right]} & {[]} & {[]} \\
{[]} & {\left[w_{l s, 2}^{1}\right]} & {[]} & {[]} & {[]}
\end{array}\right]
$$

3- Connection branch delay matrices input_delay and layer_delay: 
These two genotype matrices carry the delay information for every connection branch in the network, as described below.

Delays between inputs and nodes (input_delay): this map is similar to input_weight in structure, but its elements show the ascending delays associated with corresponding branches. For instance, the input_delay matrix of our example network will be a matrix of delay vectors as follows

$$
\text { input_delay }=\left[\begin{array}{ccc}
{\left[d_{i 1,1}^{1} d_{i 1,1}^{2} d_{i 1,1}^{3}\right]} & {\left[d_{i 2,1}^{1} d_{i 2,1}^{2} d_{i 2,1}^{3}\right]} & {[]} \\
{[]} & {\left[d_{i 2,2}^{1}\right]} & {[]} \\
{[]} & {[]} & {[]} \\
{[]} & {[]} & {[]} \\
{[]} & {[]} & {\left[d_{i 5,3}^{1}\right]}
\end{array}\right]
$$

Delays between nodes (layer_delay): this map is similar to layer_weight in structure, but its elements show the ascending delays associated with corresponding branches. For instance, the layer_delay matrix of our example network will be a matrix of delay vectors as follows

$$
\text { layer_delay }=\left[\begin{array}{ccccc}
{[]} & {[]} & {[]} & {\left[d_{l 1,4}^{1} d_{l 1,4}^{2}\right]} & {[]} \\
{[]} & {\left[d_{l 2,2}^{1}\right]} & {[]} & {[]} & {[]} \\
{\left[d_{l 3,1}^{1} d_{l 3,1}^{2} d_{l 3,1}^{3} d_{l 3,1}^{4} d_{l 3,1}^{5}\right]} & {\left[d_{l 3,2}^{1} d_{l 3,2}^{2}\right]} & {\left[d_{l 3,3}^{1} d_{l 3,3}^{2}\right]} & {[]} & {[]} \\
{[]} & {[]} & {[0]} & {[]} & {[]} \\
{[]} & {[0]} & {[]} & {[]} & {[]}
\end{array}\right]
$$

4- Darwinian weight mutations Dar_input_SD and Dar_layer_SD:

In order to avoid local minima traps one can add noise to the deterministically acquired knowledge that LMS has deposited in input_weight and layer_weight. This can be compared to alterations in non-exact knowledge transfer from parent to offspring or 
thermal cool down of system in simulated annealing if evolution monotonically decreases the noise added to connection weights. Each weight will have a corresponding Gaussian standard deviation for Darwinian mutation, which will be adjusted through the genotype objects Darwinian input standard deviation (Dar_input_SD), Darwinian layer standard deviation (Dar_layer_SD), Darwinian alternative input weights (Dar_Alt_Inp_Wts), and Darwinian alternative layer weights (Dar_Alt_Lay_Wts).

Darwinian input standard deviation (Dar_input_SD): if one considers each network weight as a point in a $\mathrm{n}$-dimensional space with $\mathrm{n}$ being the total number of branches in the network, then the Dar_input_SD describes a n dimensional hyperellipsoid centered at that point in weight space with its axes aligned with the weight space axes. The size of the ellipsoid axes determines the Gaussian weight mutation standard deviations along different directions in the n-dimensional weight space. Evolution will find the best standard deviation for the given surface to complement the deterministic LMS search. One can let the mutation standard deviation ellipsoid align itself along directions that are non-parallel to weight axes. However, that will make the evolutionary search space larger and as a result evolution time may become much longer. The Dar_input_SD for our example network will be as follows

$$
D_{\text {Dr_input_S }} S D=\left[\begin{array}{ccc}
{\left[w d_{i 1,1}^{1} w d_{i 1,1}^{2} w d_{i 1,1}^{3}\right]} & {\left[w d_{i 2,1}^{1} w d_{i 2,1}^{2} w d_{i 2,1}^{3}\right]} & 0 \\
0 & {\left[w d_{i 2,2}^{1}\right]} & 0 \\
0 & 0 & 0 \\
0 & 0 & 0 \\
0 & 0 & {\left[w d_{i 5,3}^{1}\right.}
\end{array}\right]
$$

Darwinian layer standard deviation (Dar_layer_SD): This object is the same as Dar_input_SD but for layer (node to node) connection branches. For instance, the Dar_input_SD for our example network will be as follows 


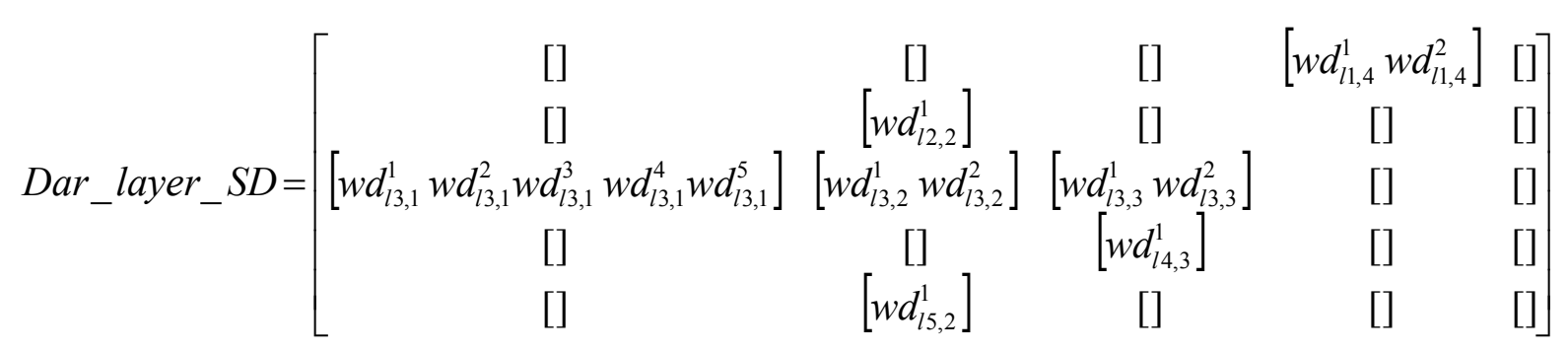

Darwinian alternative input weights (Dar_Alt_Inp_Wts): this object contains alternative input_weight matrices. Each weight matrix has exactly the same structure as input_weight. The Dar_Alt_Inp_Wts elements are derived from their counterparts in input_weight with the following Gaussian mutation:

$$
\text { Dar_Alt_Inp_Wts }(r, c)_{d}=\text { input_weight }(r, c)_{d}+\text { Dar_input_SD }(r, c)_{d}{ }^{\prime} \times N_{0,1}
$$

The standard deviation Dar_input_SD $(r, c)_{d}$ is used after its own mutation, according to Schwefel's suggestion ${ }^{86}$

$$
\begin{gathered}
\text { Dar_input_SD }{ }_{r, c}^{d}=\text { Dar_input_S }{ }_{r, c}^{d} \times \exp \left(\tau_{2} \times N_{0,1}+\tau_{1} \times N_{i 0,1}\right), \\
\tau_{2}=\frac{1}{\sqrt{2 n}}, \tau_{1}=\frac{1}{\sqrt{2 \sqrt{2 n}}} \quad(\mathrm{C} 13)
\end{gathered}
$$

$(\mathrm{r}, \mathrm{c})_{\mathrm{d}}$ indicates the $\mathrm{d}^{\text {th }}$ element in the delay vector corresponding to the connection between source $\mathrm{c}$ and destination $\mathrm{r}$. Superscript $\mathrm{d}$ denotes the branch number in a connection. Normal random number $N_{i}$ is generated afresh for each element $(r, c)_{d}$, whereas normal random number $N$ is generated only once per each offspring. The prime symbol ' indicates the recently mutated standard deviation and $n$ is the number of all current network branches. Parameters' mutations takes place before their utilization so the resulting fitness values will correspond to the actual values used. 
We need to evaluate the fitness of the resulting Darwinian search described by Dar_input_SD and Dar_layer_SD and not just one potential lucky mutation. The correct shape of the hyper-ellipsoid described by the Dar_input_SD Dar_layer_SD matrices describes the perception of the performance surface by the evolved network (see figure 27). Based on what was explained, one can evaluate the fitness of a network by averaging the fitnesses resulting from different starting points within the hyper-ellipsoid determined by Dar_input_SD and Dar_layer_SD Gaussian mutations. The function determining the number of corresponding alternate weight sets or starting points should take the size of weight mutation hyper-ellipsoid into account. In order to save time, this simplified linear function was created and used in GETnet:

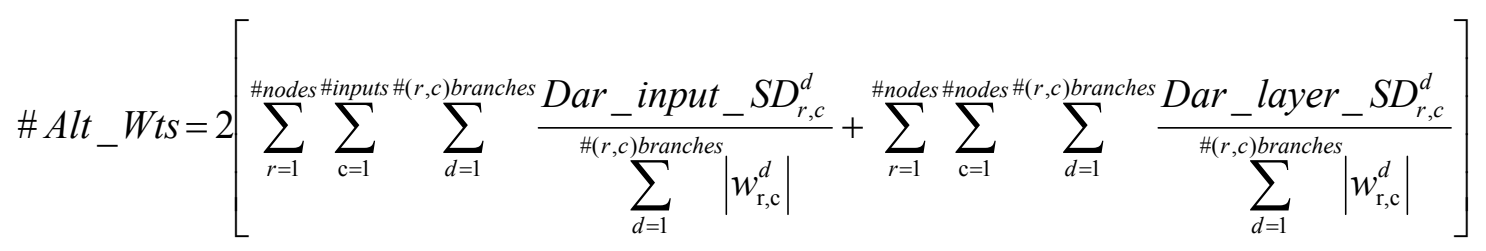

The above formula simply takes into account the sum of the ratios of the Gaussian mutation standard deviations to the magnitude of corresponding weights throughout a network. This means that bigger search radii will get more random shots to evaluate their search field. The range of the above function is clamped at 1 and 10 in order to keep the evaluated extra weight sets and consequently training time within a manageable size. This function can be replaced with any other function with a better approximation of the stochastic search domain if enough computing power is available. The network saves all these alternative weights under Alt_Inp_Wts in the genotype. However, GETnet selects the best weight (in terms of evaluated performance) as the active weight set, which is the basis of the Lamarckian evolution and Baldwin effects. Up to 9 other weights remain dormant in Alt_Inp_Wts. This is similar to a multiple (semi) randomized starting point technique for neural network and enhances the network performance even further. 
Darwinian alternative layer weights (Dar_Alt_Lay_Wts): This object contains alternative layer_weight matrices. The structure is similar to that of Dar_Alt_Inp_Wts but for the layer weights.

$$
\begin{gathered}
\text { Dar_lay_S } D_{r, c}^{d}=\text { Dar_lay_S } D_{r, c^{\prime}}^{d} \times \exp \left(\tau_{2} \times N_{0,1}+\tau_{1} \times N_{i 0,1}\right), \\
\tau_{2}=\frac{1}{\sqrt{2 n}}, \tau_{1}=\frac{1}{\sqrt{2 \sqrt{2 n}}} \quad(\mathrm{C} 15)
\end{gathered}
$$

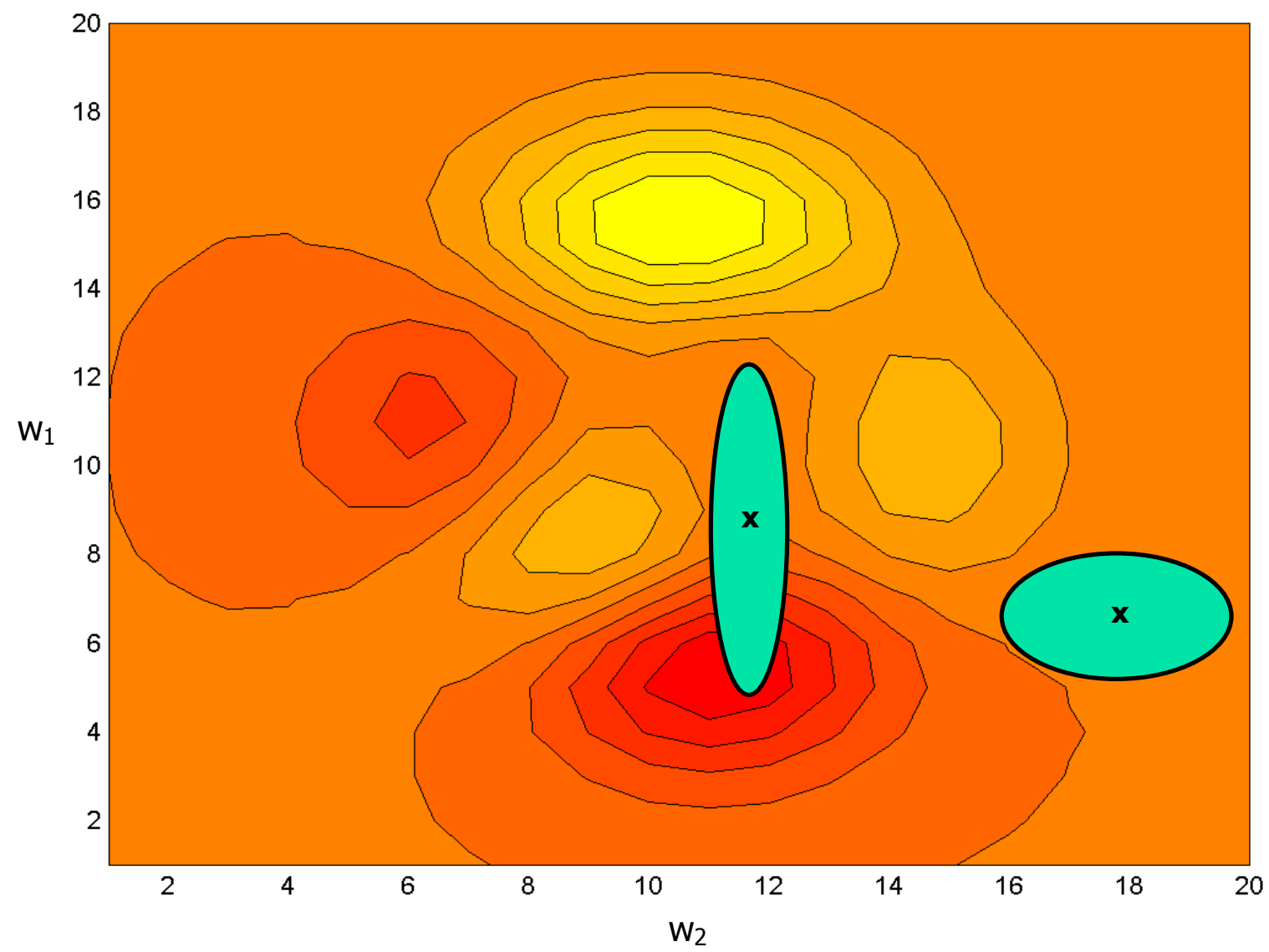

Figure 27 A hypothetical performance surface in a 2-D weight space. Ellipsoids show 2 different evolved stochastic search regions around deterministic optima marked with $\mathrm{x}$. 
$\mathrm{n}$ is the total number of weight branches. These Alt_Lay_Wts weight sets along with Alt_Inp_Wts are saved in the genotype, besides the best set which is chosen as the active weight set of the individual.

GETnet and the Baldwin Effect: It is important to note that the aforementioned mechanism can give rise to what is known as the "Baldwin Effect"97,98 in GETnet. In short, the Baldwin effect states that the ontogenetic characteristics (acquired during individual's lifetime) can eventually affect the phylogenetic (lineage) characteristics and thus guide the evolution by some kind of indirect Lamarckian transmission through evolutionary pressure. Baldwin effect can be seen in evolutionary systems that can perform local search (such as the hybrid training system of GETnet) and can result in faster learning. The phenotypical plasticity of a learning system can increase the fitness of an individual by ontogenetic acquisitions (e.g. SCG local learning in GETnet). The facilitation of the acquired phenotypes by some genotypes, which otherwise might be useless without local search and ontogenetic learning, is the first phase of the Baldwin effect. However, local searches (such as gradient descent learning) are expensive and consume resources (limited learning time in case of GETnet). Now if these desired phenotypes that start to appear in the population by local search and learning are created in some offspring by virtue of evolutionary stochastic processes (Gaussian weight perturbations in Dar_Alt_Wts facilitated by the best weight selected from Alt_Inp_Wts and Alt_Inp_Wts), then those with such inherited superior weights (co-adapted alleles) do not need to waste precious limited search time to find the already available better starting point. Especially in the event of shrinking search hyper-ellipsoids defined by Dar_inp_SD and Dar_lay_SD in conjunction with the limited number of multiple starts within the search range as defined by the saturating function (C14), this effect will be assisted by fine-tuning of the multiple starts within the narrowly guided stochastic search. These fitter and faster offspring (good mutation, smaller search radius, faster convergence to locally available optima and more relative search time) will dominate the population by increasing selection pressure on the rest of population that lack these characteristics through GETnet's temporal MDL. Please note that the inheritance of co- 
adapted alleles is built into the GETnet through noisy Lamarckian transfer of the bestlearned weight set from parent to offspring in the Evaluate module (see the description of the module below to see how the best weight set is set as active). This is the second and last phase of the Baldwin effect.

GETnet Noisy Weight Transfer vs. Simulated Annealing: One can see resemblances and parallels between the random search within the hyper-ellipsoid that adds a Gaussian noise to the inherited weights and simulated annealing, in the sense that they both utilize some controlled random search in free variable space to avoid local minima. However, GETnet's noisy weight transfer is arguably superior, especially for adaptive environments:

a) Simulated annealing assumes a static error landscape, with the chances for bigger leaps reducing in time as the system temperature decreases. The static performance landscapes used in the given simulations here lead to smaller search radii since as the population moves towards an optimum, smaller search domains yield a better evolutionary advantage. However, this behavior was not scheduled but the evolution found it to be the best approach, thus GETnet effectively invented a simulating annealing type of algorithm by itself. However, if the performance landscape changes with time and a new optimum appears outside the current random search hyper ellipsoid, then the mutants with bigger search radius will have an explorative advantage and thus will prevail since they can throw the operation point towards the new optimum basin. Thus under such dynamic circumstances, it is conceivable to see expanding rather than shrinking random search hyper ellipsoids and better coping with a changing environment.

b) Simulated annealing operates on finite discrete state vectors, whereas GETnet's noisy weight transfer operates on real valued vectors. Simulated annealing-based statistical neural networks such as Boltzmann machines (please see section B, background) also have finite states. 
c) GETnet's noisy weight transfer is augmented with gradient descent search, and the balance between these two mechanisms is found through standard deviation matrices through evolution.

d) Last but not the least, simulated annealing is not automatic since it needs human expert to design a cooling scheduling for it, i.e. it suffers from the "baby sitting" problem.

4- Other structural genotype:

Strategy Parameters: Four other major evolvable parameters are used to find the appropriate temporal network structure. These strategy parameters are prune_SD, node_mutation_SD, connection_mutation_SD, and delay_mutation_SD. Being general structure parameters, they are evolved in their own space with $n=4$ in Schwefel's mutation formula. Pruning parameter prune_SD is the standard deviation of the pruning threshold that is mutated with a Gaussian perturbation. The final product is stored in the genotype parameters prune_threshold. Pruning starts its operation from input_weight and layer_weight. More details will be given in the description of the modules Prune and Mutate.node_mutation_SD is the standard deviation for the Gaussian mutation that changes the number of existing nodes in a network. connection_mutation_SD is the standard deviation for the Gaussian mutation that changes the number of existing node to node connections in a network. That is, it operates on input_connect and layer_connect. delay_mutation_SD is the standard deviation for the Gaussian mutation that changes the number of existing delay branches in the network. That is, it operates by increasing or decreasing the length of vector elements in input_delay, layer_delay, input_weight, and layer_weight. More details about node, connection, and delay mutations will be given in the description of the module Mutate.

Non-strategy parameters: There are other genotype contents that are not subject to evolution but keep track of different behavioral properties. They include training parameters epochs, goal, time, min_grad, and max_fail, among the others. They will be 
described in module Evaluate and GetCommittee. Other record keeping components include parent tags to enable backtracking a lineage, performance error, and average training time.

\section{Execution: GETnet Module}

GETnet starts from a main module by the same name. It accepts training input and target sequences as well as validation information. It normalizes all the teacher data linearly from -1 to 1 for ease of training. The outputs are denormalized using the inverse of this linear transform at the end. GETnet accepts input sequences of any dimensionality $\mathbf{X}(\mathrm{t})$ (any multi-dimensional signal) along with a single or multi-dimensional target signal $\mathbf{Y}(\mathrm{t})$ and will try to find the corresponding nonlinear temporal mapping $f$ so that

$$
\mathbf{Y}=f(\mathbf{X}) \quad(\mathrm{C} 16)
$$

The evolved temporal model approximates the relationship $f$ in $(\mathrm{C} 16)$ by minimizing the MSE for unknown experimental rule $f$. The approximant to the function $f$ will consider the history of $\mathbf{X}(\mathrm{t})$ through long and short-term memory structures. GETnet also asks for a validation subset of training data (please see Evaluate module for more explanation). Validation data will be used for performance evaluation in the evolution phase since fitness scores are based only on validation data or validation-based early stopping. This way the evolving networks will be evaluated based on the ultimate goal, their generalization performance. Another option is mixing training and unseen data for validation so the networks will be graded both for training and generalization quality. In this case the early stopping mode in Evaluate maybe deactivated.

Initial population is another parameter passed to the GETnet module. Larger populations are better since they increase the breadth of evolutionary search, but that will increase evolution time linearly so a reasonable value should be chosen based on 
available time and computing power. All of the experiment results given here were obtained using a population size of 25 . Other input parameters are a feed-forward-only switch (in order to limit evolution to feed-forward subsets of answers when a faster, nolong term memory solution is desired), minimum desired MSE precision, a time-out value, and the number of different examples provided for training and validation (for batch processing). Please note that at the time of preparation of this dissertation Mathworks was recommending the batch mode not to be used with their neural network toolbox in conjunction recurrent networks (version 4 for Matlab 6.5). Thus serial concatenation of different sequences was used when needed to avoid potential problems.

During its execution, GETnet goes through the following evolution steps:

- Create the initial population (Genesis).

- Evaluate the initial population.

- While (desired goal has not been attained):

- offspring=Mutate(parents).

- Evaluate offspring.

- Select between parents and offspring.

- Create a Committee of further trained last generation.

The GETnet module assigns the number of mutant offspring that each parent can have according to the roulette wheel selection described in the background section B4-1. Since roulette wheel selection uses the values produced by the Evaluate module, its description will be given in the Evaluate module section.

\section{Genesis Module}

The module Genesis is responsible for creation of the first generation (initial population). It creates $\lambda$ nonlinear recurrent time delay neural networks almost randomly and with a minimum number of heuristic guidelines, such as each node should have at 
least one input and one output, and that zero delay loops should be avoided. A switch can force the optional feed-forward structure (not used in any of the simulations). The network can have 1 to $\mathrm{M}$ inputs and 1 to $\mathrm{N}$ outputs, with $\mathrm{M}$ and $\mathrm{N}$ being detected automatically from teacher data. The initial number of neurons is chosen to be from num_outputs (good for linearly separable problems) to (4×num_inputs)+num_outputs. $2 \times$ num_inputs is enough for at least one closed decision surface, and (4×num_inputs)+num_outputs is enough for at least two disjoint closed decision surfaces. These are chosen as reasonable initial ranges and can change through evolution if the need arises to obtain the required number of disjoint decision regions.

A maximum delay depth of 20 on any connection branch and a maximum of 10 initial parallel delays branch per connection is chosen in Genesis. These are approximate initial lower limits since for instance, if maximum branch number for a connection is larger than its maximum delay depth, the delay depth will be increased to accommodate the extra parallel branches and avoid redundant same-delay branches. Again, these are initial delay structure values and will be evolved to reach the required memory through FIR and IIR subunits and paths.

Lamarckian and Darwinian Evolution: As mentioned during description of Dar_input_SD and Dar_layer_SD, GETnet networks simultaneously evolve under two evolutionary forces, namely Lamarckian and Darwinian. The garnered knowledge under backpropagation (i.e. acquired characteristic) is partially passed on to the next generation through input_weights and layer_weights. This is similar to the knowledge transfer from parent to offspring through an educational system in a society of evolving species, creating further phenotypical resemblance between parent and offspring and facilitating the Baldwin effect. In this case, since the reproduction is asexual and mutation-based, the transfer is one to many, from parent to its direct mutant offspring. This transfer is not exact since it is distorted by a Gaussian noise whose standard deviation is subject to evolution (see definition of Dar_input_SD and Dar_layer_SD). This process creates reasonable room for new generation stochastic plasticity by giving them the ability to 
explore the landscape around the inherited starting point. This can be viewed as some sort of multiple starting point technique that enhances neural network performance. The zero mean Gaussian distribution of additive weight noise helps the general weight resemblance (i.e. content or knowledge similarity) between parent and offspring. The offspring will go through deterministic partial gradient descent training from these inherited noisy starting points. In addition, the added noise will help shake the network out of local minima. It can be shown that the effects of adding noise are similar to adding noise to the target values to improve generalization and convergence ${ }^{99}$. Since the standard deviation itself is an evolutionary parameter, it can adapt itself to the peculiarities of the error landscape.

Genesis uses two uniformly distributed random numbers, from 0 to 1 , to determine input-to-network and node-to-node connection densities. To give the network the advantage of being able to explore all novel structures, very few assumptions and rules are imposed. One trivial rule is making sure that there is at least one input and one output connection for each node, whether it is an input from another node or an external input, or an output to another node or an external output. The feed-forward switch is imposed by forcing the binary layer_connect matrix upper triangle and main diagonal to remain zero. Furthermore, for feed-forward initial connectivity, the last $\mathrm{N}$ nodes are designated to be the output nodes. The default recurrent architecture does not need this stipulation since there is no forward ordering and thus an output layer or node(s) need not appear at any specific location. The recurrent structure is the default and preferred mode since it can fall back into feed forward by deleting its feedback loops through mutation and pruning as needed. The feed forward switch is just a shortcut when one needs a faster convergence under special circumstances (e.g. no need for longer term memories and faster evolution) and should generally be avoided to let the evolution choose the topology, as is the case for the simulations presented in this document. Genesis also enforces a minimum loop delay of 1 step to avoid the impossible zero-delay loops. Small initial random values for Dar_input_SD, Dar_layer_SD, prune_SD, prune_threshold, 
node_mutation_SD, connection_mutation_SD, and delay_mautation_SD are chosen in this module, as described below.

1. Node deletion/addition node_mutation_SD:

This is the standard deviation for a Gaussian mutation dictating what percentage of nodes will be added or deleted by the Mutate module. This parameter is an evolution strategy and subject to mutation itself. A small uniform random initial value (up to 0.2 ) is chosen here. Note that in long run, the search is not usually sensitive to these starting points since they will be adjusted during the course of evolution. However, to speed up the process and keeping the general phenotypical resemblance of offspring to parent rule of thumb in mind, this initial value is chosen to be generally less than the next two general strategy mutation values connection_mutation_SD and delay_mautation_SD since changing the number of nodes is usually a more drastic and thus less function-preserving mutation. When adding a node, GETnet uses the network's branching statistics to further preserve general connectivity resemblance (see Mutate).

2. Connection deletion/addition connection_mutation_SD:

This is the standard deviation of the Gaussian mutation that determines the percentage of connections that will be added or deleted. Connection deletion will take out all the constituting delay branches, while the addition will create delay branches that conform to the general network statistics (see Mutate). The uniformly distributed random initial value for this parameter is higher than the one for node mutation (0.4), since connection mutation is usually less disruptive.

3. Delay branch addition/deletion delay_mautation_SD:

This strategy parameter is the standard deviation of the Gaussian mutation that determines the percentage of network delay branches to be randomly added or deleted. The uniformly distributed random initial values for this parameter is 
higher than the others $(0.8)$, since changes in the parallel branches are relatively less disruptive.

4. Pruning threshold standard deviation prune_SD:

This Gaussian mutation will determine the change of prune_threshold, the connection deletion threshold (see Prune). prune_SD is the strategy parameter that is subject to mutation, and the prune_threshold is initialized with small uniform random positive numbers (between 0 and 0.05 ) here.

The above four standard deviations are later mutated in their own strategy space using Schwefel's formula with $\mathrm{n}=4$ (see (C21) to (C25)). Genesis calls the NewTDNN module (new time delay neural network) for creation of the required initial networks according to the parameters described above. NewTDNN creates the network object but the genotype contents are created and inserted by Genesis. Later modules such as Prune and Mutate access and change this genotype as needed.

\section{NewTDNN Module}

NewTDNN accepts input connection matrix input_connect, input delay matrix input_delay, input weight matrix input_weight, layer connection matrix layer_connect, layer weight matrix layer_weight, and output connection vector output_connect, and returns a new time delay neural network object with the specified parameters. All neurons have bias connections and their input range is set to -1 to +1 since GETnet normalizes training data in this range at its entry point for better convergence. NewTDNN also sets the weight by the Nguyen-Widrow initialization method so neurons will be initialized in their active region for higher initial gradient since the sigmoidal activation function has maximum derivative at the middle of its active region. Mutate and Prune force the weights by specifying input_weight and layer_weight. This module also sets the network's performance function to mean squared error (MSE). 
The training method is the scaled conjugate gradient (SCG), an advanced LMS method. SCG is used because of its generality (according to Matlab's benchmark results reported in neural network $\mathrm{v} 4$ help documents), speed (superlinear convergence rate), reduced memory requirements compared to other second degree methods especially when it is used with back propagation through time (BPTT), and performance on sharp error surface valleys that may be produced by GETnet's favoring of compact solutions. SCG is based upon the general conjugate gradient optimization methods and uses second order information from the neural network. However it requires only $\mathrm{O}(\mathrm{N})$ memory usage, where $\mathrm{N}$ is the number of weights in the network. SCG yields a speed-up of at least an order of magnitude relative to regular backpropagation. SCG is fully automated, which is in accordance with GETnet's "no baby sitting" philosophy. Other gradient descent methods depend on parameters which have to be specified by the user, and usually no theoretical basis for choosing those parameters (e.g. learning rate and momentum constant in backpropagation). Since GETnet tries to optimize a large number of parameters, conjugate gradient $(\mathrm{CG})^{100,101,102,103}$ methods are more practical. However, other CG algorithms suffer from problems that SCG avoids. These including the time consuming line-search, which other conjugate gradient algorithms use to find a suitable step size by utilizing the Levenberg-Marquardt method ${ }^{100}$ for scaling the step size. The direction of search is determined from a second order approximation of the error function which avoids the $\mathrm{O}\left(\mathrm{N}^{2}\right)$ memory complexity and $\mathrm{O}\left(\mathrm{N}^{3}\right)$ time complexity for calculating the Hessian matrix. GETnet, with its temporal MDL policy, tends to find smaller solutions and thus simpler networks. This increases the possibility that the weight space contains long ravines characterized by sharp curvature. While backpropagation is inefficient on these ravine phenomena, it is shown that SCG handles them effectively. Unlike the other conjugate gradient methods, SCG is convergent for non-quadratic error surfaces (please see appendix A). The specifics of both theory and implementation of SCG can be found in Moller's original paper ${ }^{104}$. 
In GETnet, the following default SCG parameters are used:

Maximum validation failures max_fail $=5$ (for early stopping, please see "Stopping the Training" in B3-3 and figure 14).

Sigma (for change in weight for second derivative approximation) $=5 \times 10^{-5}$.

Lambda (for regulating the indefiniteness of the Hessian) $=5 \times 10^{-7}$.

The above default values were experimentally found to be satisfactory. Moreover, they are used in partial training and within the stochastic evolutionary search, which makes the overall process less sensitive to their precision.

\section{Evaluate Module}

After creation of the first generation (i.e. initial parents), Genesis needs to estimate their fitness before they enter the evolutionary loop by calling Evaluate. Once inside the evolution loop, Evaluate is applied to each generation to find the fitness and reproduction chance of each individual (see figure 25). These fitness scores are used to determine chances of reproduction and survival. Evaluate accepts a generation of networks as well as training and validation data sets through the GETnet module. Evaluate then partially trains each network in the given generation and determines their fitness score, which is written into the network genotype. There are three aspects which are further described in the sections below. (1) The data used for training and validation can be defined in different ways. (2) The time allotted for partial training before evaluation is controlled by a new method for regularization called temporal MDL. (3) The fitness score is calculated for each parent and the offspring are generated based on the roulette wheel method of selection. (4) The other standard training termination policies are given. 
The fitness score itself is simply $\frac{1}{\text { mean }(M S E)}$. MSE is averaged over the main and alternative network weight sets to evaluate the random search ranges as defined by Dar_input_SD. Dar_layer_SD, and (C14) (please refer to their descriptions earlier in section C2). The weight set (i.e. input_weight and layer_weight) with the best score is set as active and is transferred to the offspring.

Temporal MDL: Evaluate implements a new, more realistic version of minimum description length (MDL) as a regularization mechanism. Time is usually the most important factor in computer applications. Regularization is necessary for helping networks' generalization capabilities by penalizing bigger, more complex solutions ${ }^{105}$. Furthermore, temporal agility has been specified as an indicator of machine intelligence $^{106}$. Traditional regularizations such as Akaike information criteria ${ }^{107}$ (AIC) do not measure either the neural network actual implementation complexity or its actual time complexity. The usual approaches such as counting the number of weights in a network do not yield a direct measure of model complexity and thus model variance. For instance, AIC does not differentiate between different network connections while the function of a weight in input is very different from that of a weight in a hidden layer. One can hypothesize that since the actual training time for a network on a computational platform is proportional to its size and complexity, then penalizing each offspring according to its CPU time is one method to perform regularization. On the other hand, since we will be favoring parsimony in terms of the actual time on a given platform, we will produce solutions that are pragmatic and best fit for the available computing technology. Favoring the less time complex solutions will lead to a temporal MDL solution that is the equivalent of Occam's razor in digital computing. These faster networks can be considered to be more intelligent as well. The implementation of this new method for time-based regularization is explained below.

The $3^{\text {rd }}$ quartile-size network's average training time for five epochs is the basis for the desired regularizing pressure. A five-epoch training time is almost enough for a nominal large network ( $3^{\text {rd }}$ quartile) to descend towards a minimum on the error surface, while it will be plenty for smaller networks to take their time and lower their performance 
error to the extent that SCG can. For this purpose, first each generation's networks are sorted according to their total number of connection branches in ascending order. Then the network that is on the $3^{\text {rd }}$ quartile slot is chosen and is trained for five epochs using its main as well as all alternate weights. The average time is then set as the maximum SCG training timeout parameter for all the offspring in the current generation. This gives the smaller networks an advantage for improving their performance given that their configuration and size is capable of doing so. Thus the smaller and faster solutions will have a higher chance in roulette wheel selection (please see formula $(\mathrm{C} 17)$ and its explanation) and in the case of feasible simple and compact solutions, they will dominate future generations. This way parsimony, and thus MDL, and generalization through penalizing complexity are encouraged. If the smaller networks cannot achieve higher performance, i.e. when the given problem cannot be solved by the compact networks, then the five-epoch average $3^{\text {rd }}$ quartile training time will provide the more complex networks in the evolution pool with the chance to demonstrate their performance and gradually shifts the average generation size towards the larger solutions. However, the shift will stop when an acceptable balance between size and performance has been reached since the described MDL mechanism always exerts a pressure towards parsimony of answers. Pruning will also act towards this goal, which will be described later.

Pre-evaluation Training Modes: In order to achieve better generalization, Evaluate calculates MSE using validation data. The validation data can be utilized in two different modes:

1- Training does not use early stopping through validation data, but the score is based on the network's validation data MSE after pruning. This way, both the generalization and pruning parameters are included in the final score and are thus subject to evolutionary selection. One can also concatenate training data and the unseen validation data for validation to evaluate both network generalization and network trainability. This is the default method but may have a slower 
convergence due to the bigger search space that includes pruning search parameters. Please see Mackey-Glass prediction task for an example.

2- Training does use early stopping. The fitness score will take into account the generalization by stopping the MSE from delusive improvements when the validation MSE increases for 5 epochs (as set by earlier described max_fail parameter). This mode is used when one wishes to bypass pruning so GETnet will converge faster in a smaller search space. In this case, GETnet will rely on mutation and MDL forces for parsimony. This mode can be used for large multidimensional data that may consume a lot of time. Please see fingerprint vitality test case for an example.

Roulette Wheel Selection: The inverse of the MSE (i.e. the fitness score) is used for this selection scheme. This method was described in the background section B4-1. Since the fitness function is defined as the inverse of the validation MSE as given by the Evaluate module, then (B104) for GETnet can be written as:

$$
P_{\text {parent }}\left(\text { net }_{i}\right)=\frac{\text { EvaluateMSE }\left(\text { net }_{i}\right)^{-1}}{\sum_{j=1}^{\text {population\# }} \text { EvaluateMSE }\left(\text { net }_{j}\right)^{-1}}
$$

Where EvaluateMSE $\left(\right.$ net $\left._{i}\right)$ is the MSE of the $\mathrm{i}^{\text {th }}$ network in the population obtained according to the methods described earlier. To understand (C17) better, one can imagine a pie chart with unit area and population\# slices. Each slice has the area $P_{\text {parent }}\left(n e t_{i}\right)$. The pie chart then receives population\# random shots. The number of offspring for each individual $i$ is then the number of shots that its slice has received. That is, the larger $P_{\text {parent }}\left(\right.$ net $\left._{i}\right)$, proportionally the higher the chance of producing more offspring. 
Additional Termination Policies: After finding the mean time for the $3^{\text {rd }}$ quartile network for 5-epoch training, Evaluate stops the partial training for each network in the current generation when any of these conditions occur:

1- Maximum amount of time, based on the $3^{\text {rd }}$ quartile five-epoch training time, is exceeded (see temporal MDL description above).

2- Error has been minimized to 0 (extremely unlikely).

3- The gradient has fallen below 0.025 , which implies no significant gradient descent will takes place (this number was chosen experimentally and based repeated observations from test runs).

4- Validation MSE has increased more than max_fail times (see pre-evaluation training mode 2 above).

Evaluate finally returns partially trained and pruned networks with their corresponding fitness scores.

\section{Prune Module}

This unit prunes any given temporal network according to the pruning threshold encoded into network's genotype. Prune also calls Dependency and NewTDNN modules for their services. The pruning process reduces model variance even further by eliminating weaker connections. This process is reminiscent of synaptic pruning of overconnected young brains both in humans and other vertebrates ${ }^{108}$, reflecting activity or energy based synaptic elimination.

Prune browses all the available connection branches mapped in input_weights and layer_weights, and finds the relative importance of each incoming weight branch i from source $\mathrm{c}$ to destination $\mathrm{r}$ by 


$$
\operatorname{IMPORTANCE}\left(w_{r, c}^{i}\right)=\frac{\left|w_{r, c}^{i}\right|}{\sum_{c=1}^{\# \text { \#ndes } \#(r, c) \text { branches }} \sum_{d=1}^{d}\left|w_{\mathrm{r}, \mathrm{c}}\right|}
$$

if this relative synaptic strength of $1^{\text {th }}$ branch from node $\mathrm{c}$ to node $\mathrm{r}$ with respect to other incident branches to node $\mathrm{r}$ falls below the pruning threshold prune_threshold, the corresponding branch along with its delay will be deleted. More advanced pruning techniques such as optimal brain damage described in background section can be used as well. However, that would increase the time complexity of GETnet.

Prune checks to see if it renders a given network useless (e.g. deleting an output or all inputs, or in the worst case, all the connections) and will return an empty object if so. Pruning the only branch in any connection deletes that connection. Prune then checks to see whether it is disconnecting all of a node's inputs from other sources or its output (self feedback obviously does not count as the sole input). If so, then the node should be deleted. Dependency is invoked next to see whether such deleted nodes were the bases of other nodes, in which case the whole chain should be deleted (the concept of dependent nodes and their bases will be explained in the module Dependency). Prune then checks to see if all external inputs to the network or any of its external outputs are disconnected, in which case the network is useless and will be deleted and an empty object is returned. All the genotype structure maps i.e. input_connect, layer_connect, output_connect, input_weights, layer_weights, ,input_delay, layer_delay, Dar_Alt_Inp_Wts Dar_Alt_Lay_Wts, Dar_input_SD, and Dar_layer_SD are adjusted accordingly. The new pruned network is instantiated by calling NewTDNN.

The heuristics for excluding nonfunctioning networks from being evaluated are trivial and will not limit evolution's degrees of freedom since a nonfunctioning network stands no chance against even the worst performing individual (zero offspring with roulette wheel selection). However, deletion of nonfunctioning networks will reduce the burden of evolution's extensive search. 
As mentioned earlier, Evaluate calls Prune. Both the prune_threshold and its evolving standard deviation prune_SD are a part of the evolutionary strategy influencing network parsimony. If the evolution or deterministic training weakens any connection below this threshold, then Prune deletes that connection. This also helps in reducing the network description length that might in turn help network's generalization and execution speed, which gives a network double evolutionary advantage by allowing more training epochs in the allotted time.

It was observed earlier that Prune deletes networks that are rendered nonfunctional. This makes GETnet's evolution not to be strictly $\mathrm{EA}(\lambda+\mu)$. For instance, $\lambda$ can drop for a parent generation based on the effect just described. $\mu$ can also drop below its initial value because of a similar effect during mutation-based reproduction in the Mutation module described later. Thus one can describe GETnet's variable-size evolution policy as

$$
E A(\Lambda(n)+M(n)), \max (\Lambda(n))=\lambda, \max (M(n))=\mu
$$

where $n$ is the generation counter and $\Lambda(n)$ and $M(n)$ are the population of parent and offspring for the current generation. Here we set $\lambda=\mu=$ initial population in GETnet's entry point. It must be mentioned that mechanisms have been built into the Genesis module that avoid degenerative conditions for the initial population, making sure that the evolution loop starts with a working non-empty set of parents.

\section{Dependency Module}

This module finds node dependencies needed for correct node deletions by the Mutate and Prune modules. It accepts network connection maps input_connect, layer_connect, and output_connect, and returns the list of nodes that are directly or indirectly dependent 
on each network node, i.e. dependents vs. their bases. Dependency first finds the direct reliance of each node $d$ on the other nodes $b_{i}$ or $b_{o}$ by finding out:

1. If there is a node such as $b_{i}$ that is the only sources of this node $d$, or

2. If there is a node such as $b_{o}$ that is the only destination of the node $d$. In either case, $d$ is dependent on $b_{i}$ and $b_{o}$ since without them, $d$ serves no purpose (direct dependency).

In its second pass, Dependency makes a series of logical deductions on interconnected direct dependencies through a chain of hypothetical syllogism (transitive dependencies):

$$
(p=D(q) \wedge q=D(r)) \rightarrow p=D(r)
$$

where $x=D(y)$ is the predicate form stating ' $\mathrm{x}$ is directly dependent on $\mathrm{y}$ ', as described in Dependency's first pass. This phase yields the rest of node dependencies, which we shall call indirect. These dependencies are returned in form of a binary matrix. This matrix is used in the Prune and Mutate modules to delete chains of nodes which have been affected by pruning or mutations and made useless.

\section{Mutate Module}

The unit Mutate is in charge mutation-based (asexual) reproduction. As mentioned earlier, crossover is not recommended for evolving neural networks. These mutated networks explore strategy, structure, weight, and delay spaces in Darwinian part of the evolution. Mutate follows Schwefel's guidelines for Evolutionary Strategies (ES), making the given network change parameters through additive zero mean Gaussian perturbations.

Mutate returns either the mutated network or an empty object if mutation renders the network unusable. To further help genotypical and eventually phenotypical linkage of 
a lineage, besides deterministic training with the same teacher data, the following heuristics are applied:

- In case of additions (network expansion), through helper statistical data gatherer units Stat and StatN, Mutate uses the network's overall structure for adding sub-structures such as connections, delay branches, and nodes in a way that would not deviate drastically from that of the parent. This helps genotypical and eventually phenotypical linkage of the lineage.

- In case of deletions (network reduction), while trying to perform reduction operations randomly, GETnet tries to find reasonable candidates for a pool of random selections. The philosophy behind this heuristic is as follows: in a sense, by testing the fitness of a mutant offspring, the evolution is calculating sensitivity of the individual's overall fitness score with respect to a perturbed parameter. If the parameter creates an unrepresentative change (e.g. disconnecting an output instead of many other available and nondestructive elimination candidates), the ratio of fitness change with respect to this parameter change will be unrepresentative. GETnet's Mutate module tries to avoid these extreme cases in order to speed up the process and avoid obvious dead-ends.

The above heuristics are among the unique contributions of GETnet for evolutionary lineage continuity.

One needs to mutate each parameter before using it. First the standard deviations prune_SD, node_mutation_SD, connection_mutation_SD, and delay_mautation_SD are mutated according to Schwefel's method with the following parameters

$$
\tau_{2}=\frac{1}{\sqrt{2 n}}, \tau_{1}=\frac{1}{\sqrt{2 \sqrt{2 n}}}, \quad n=4
$$


Then structure and delay maps are mutated accordingly. In the following formulas, $\mathrm{N}_{0,1}$ is a normal Gaussian number generated once per mutated offspring for the 4-member strategy parameter space. $\mathrm{N}_{\mathrm{i} 0,1}$ and $\mathrm{N}_{\mathrm{j} 0,1}$ are normal Gaussian random numbers generated afresh per parameter.

Structural changes occur in the following order. For all these mutations, $\tau_{1}, \tau_{2}$, and $n$ are given by (C21).

\section{1- Branch add/delete}

The following will determine the mutant's new total delay branches:

$$
\text { total_Branches }_{\text {offspring }}=\text { total_ } \text { Branches }_{\text {parent }}+\Delta \text { Branch }
$$

where

$$
\begin{gathered}
\text { delay_mutation_SD }=\text { delay_mutation_S } S D_{\text {old }} \times \exp \left(\tau_{2} N_{0,1}+\tau_{1} N_{i 0,1}\right) \\
\Delta \text { Branch }=\text { round }\left(\text { total_Branches } \times N_{j 0,1} \times \text { delay_mutation_SD }\right)
\end{gathered}
$$

When $\Delta$ Branch $>0$, mutation acts on the existing connections in layer_connect and input_connect and randomly adds that total number of branches. The corresponding weight and Darwinian standard deviation is randomly initiated from normal distributions of the other branches on the receiving node (assuming Gaussian distribution) to make these additions more homogenous. The new branch delay is randomly (uniformly) chosen to be up to twice the network maximum delay. This way the network can increase its memory depth during branch additions. 
When $\Delta \mathrm{Branch}<0$, mutation tries to randomly decrease the total branch delay depth by that amount, while staying away from single-branch connections. If the number of such connections is bigger than the number of required deletions, then the excess will be carried over to connection mutation for the resulting connection deletion.

\section{2- Connection add/delete}

The following will determine the mutant's new total connections:

$$
\begin{aligned}
& \text { connection_mutation_SD }=\text { connection_mutation_SD } D_{\text {old }} \times \exp \left(\tau_{2} N_{0,1}+\tau_{1} N_{i}{ }_{0,1}\right) \\
& \Delta \text { Connections }=\text { round }\left(\text { total_Connections } \text { parent }_{\text {C }} \times N_{j 0,1} \times \text { connection_mutation_SD }\right) \\
& \text { total_Connections } s_{\text {ofspring }}=\text { total_Connections } \text { parent }_{+}+\Delta \text { Connections }
\end{aligned}
$$

When $\Delta$ Connections $>0$, mutation acts on the non-existing connections in layer_connect and input_connect and randomly adds that total number of connections with parallel delay branches. The new connection branches' weights and Darwinian standard deviations are randomly initiated from the normal distributions of the other branches incident on the receiving node (assuming Gaussian distribution). However, the new connection's number of branches and delay depths are chosen from the means of other incident connections to the receiving node. This is because it is the job of branch mutation and not connection mutation to randomly change those values. Thus a connection memory depth change happens during connection branch mutation.

When $\Delta$ Connections $<0$, mutation tries to randomly decrease the total number of connections by that amount. In this case, $\Delta$ Connections may contain carry-overs from delay branch disconnecting reductions. Mutate does not consider critical connections. 
A connection is deemed to be critical if its deletion will leave one or more node without input from other node(s) or output to other node(s). In this case, not only the connection, but also the whole node should be taken out, making the mutation noisier. If no non-critical connections are left and Mutate has to delete such node-reducing connections, it will pass on the job to node mutation below.

\section{3- Node add/delete}

The following will determine the mutant's new total number of nodes:

$$
\begin{gathered}
\text { node_mutation_SD }=\text { node_mutation } \_S D_{\text {old }} \times \exp \left(\tau_{2} N_{0,1}+\tau_{1} N_{i 0,1}\right) \\
\Delta \text { Nodes }=\text { round }\left(\text { total_Nodes }{ }_{\text {parent }} \times N_{j 0,1} \times \text { node_mutation_SD }\right) \\
\text { total_Nodes } \text { offspring }_{-}=\text {total_Nodes }{ }_{\text {parent }}+\Delta \text { Nodes } \quad(\mathrm{C} 24)
\end{gathered}
$$

When $\Delta$ Nodes $>0$, Mutate first generates a suitable but random location where the new node will be inserted. Output locations are avoided for the sake of being less disruptive. Note that for the feed-forward mode this location cannot be after the last output node. The new nodes' numbers of incoming and outgoing connections (fan in and fan out) are calculated from the entire network averages. Number of parallel delay branches, their weight, and Darwinian standard deviations are randomly initialized from the normal distributions of the other nodes in the whole network (assuming Gaussian distribution), which makes these additions more homogenous and less disruptive. New node's branch delays are randomly (uniformly) chosen to be up to the maximum network delay. Similar to connection mutation case, the increase of this existing depth is left to branch mutation. Module Stat provides the required network statistics. The network statistics used for new node instantiation come from the state of the network before entering these successions of mutations. 
When $\Delta$ Nodes $<0$, mutation tries to randomly decrease the network size by that amount. In this case, $\Delta$ Nodes can contain carry-overs from critical connection removals. Mutate first finds node chains by calling Dependency. Then it searches all the node chains for the longest that are non-critical. That is, it tries to exclude chains that contain outputs. Mutate then chooses connected chains randomly, but in a descending order of length if possible, till the number of required nodes are deleted. If the network is shrunk to an inoperable level, Mutate returns an empty object.

\section{4- Weight mutations}

Weights are mutated according to (C13) and (C15) in their own weight space. The number of dormant weights are given by (C14). The matrices Dar_inp_SD and Dar_lay_SD should first mutate before being utilized.

\section{5- Pruning parameter mutation}

Mutate is also in charge of pruning parameter alterations, which are calculated in the four-member strategy space and recorded into the network's genotype as follows

$$
\begin{array}{r}
\text { prune_SD }=\text { prune_SD } D_{\text {old }} \times \exp \left(\tau_{2} N_{0,1}+\tau_{1} N_{i 0,1}\right) \\
\text { prune_threshold }=\text { prune_threshold }{ }_{\text {old }}+N_{j 0,1} \times \text { prune_STD }
\end{array}
$$

\section{Stat Module}

This unit is in charge of collecting global and local statistics that are used both by Prune and Mutate. It accepts a network object and returns the total number of network branches, number of parallel branches between each two nodes $(r, c)$ both in 
input_connect and layer_connect (based on network's directed connection weight multigraphs), mean and standard deviations of all synaptic weights as well as mean and standard deviation of Darwinian weight mutation standard deviations (Dar_inp_SD and Dar_lay_SD), among other things. It also returns the values for relative importance of each incident input and layer branch as described in (C18).

\section{StatN Module}

This is a stripped down and faster version of Stat that only returns the total number of parallel branches when the other statistics of Stat are not needed, such as calculation of $\mathrm{n}$ in (C13), (C15), and (C21) for Schwefel's ES mutation method.

\section{GetCommittee Module}

As mentioned during the background section, in the case of statistical independence of errors, a committee of classifiers can reduce the test data error. For GETnet, since the last generation will include the best of surviving evolved solutions, one can average their outputs to get a committee of networks, which is what GetCommittee module does. In case of a highly evolved and optimized best network, the difference between the committee and the best network outputs is usually negligible. However, especially when the evolution has not converged, the committee may yield a better test performance. Furthermore, because of the averaging action, the committee output signals may be smoother.

Being the last module of GETnet, GetCommittee further trains all the last generation networks (full training) in order to complete the partial training of the evolution phase. This module also accepts training, test, and validation input and target data (for early stopping). Other provided parameters include ideal training precision goal, batch-mode sizes, and maximum number of training epochs as a safety termination condition. GetCommittee returns both committee output based on last generation average and the best single network output and saves the final results. 


\section{C3 Simulations}

In this section, the results of three simulation tasks are presented. Each simulation starts with a problem description, followed by the simulation settings and then detailed results. A discussion concludes each simulation description, pointing out the earlier theorized characteristics of GETnet in practice.

\section{Mackey-Glass Chaotic Series 1}

This is a prediction benchmark time series with a real valued one-dimensional discrete time input signal and a one-dimensional signal as the target output. Prediction of the time series based on its history is desired. Such predictive models are useful when mathematical description of the sequence does not exist or it is incomplete. Stochastic models are usually based on linear methods which are not suitable for nonlinear processes. Neural networks are among nonlinear methods proposed for these problems. Here we will show how GETnet can find a minimal predictor network through evolution and training.

Given the properties of this series, Mackey-Glass is used to benchmark time series processing capabilities of many neural networks ${ }^{109,110,111}$. This series is recommended by IEEE Neural Networks Council Standards Committee Working Group

on Data Modeling Benchmarks as a reference for comparisons ${ }^{112}$.

\section{Problem Description}

Mackey-Glass is a chaotic, non-periodic (pseudo-periodic), non-convergent univariate time series when its initial condition is set to $x(0)=1.2$ and its depth parameter to $\tau=17$. The series' behavior is very dependent on the values of the initial condition and the parameter $\tau$. The Mackey-Glass series is defined by the following differential 
equation, which was first introduced as a model for white blood cell counts to describe the onset of leukemia ${ }^{113,114}$.

$$
\frac{\mathrm{d} x(t)}{\mathrm{d} t}=\frac{0.2 x(t)(t-\tau)}{(1+x(t-\tau))^{10}}-0.1 x(t)
$$

The proposed tasks are 6 and 36 step predictions for the series. The former was chosen since it is a popular benchmark among researchers in the field. The latter prediction was chosen since it is a deep prediction and can test the capabilities of the evolved network based on wide-gap sampling.

\section{Data and Simulation Settings, 6-Step Prediction}

The first 1500 points of a 6-step sampled Mackey-Glass series with $\tau=17$ (MG17) was used in this simulation (please refer to the generator $\mathrm{m}$ files and data source in the accompanying $\mathrm{CD}$ or the following FIRnet reference). The data itself was obtained from Eric Wan's benchmark collection of temporal data for FIRnet ${ }^{115}$. The sought task is a 6-step prediction. That is, given MG17(n), the network is to predict the value of MG17(n+6). Note that since the data is resampled every 6 steps, each consecutive sample counts for a 6-point leap in MG17. That is, $x(n+1)$ refers to MG17(n+6), and so forth. Thus GETnet has to find a model to estimate $f(x+1)$ from $\{f(x), f(x-1), f(x-2), \ldots\}$.

The data is divided for training, validation, and testing as follows. The first 500 samples (1 through 501 for input and 2 through 502 for target) are used for the SCG partial training during the evolution loop, and the first 1000 samples (1 through 1001 for input and 2 through 1002 for target) are used for the corresponding validation score as derived by Evaluate module. The overlap of the seen first half and the unseen second half of the validation data is intentional. The contribution of the first half to the score accounts for the training quality of the network while the effect of the second half measures its generalization ability. Note that the training and validation data can be different in 
evolution and the final complete training in GetCommittee phase. Furthermore, one can choose not to use the aforementioned technique in combining training and validation scores for fitness evaluation and instead use the training error with early stopping. In this case, the generalization capabilities of the network will be reflected in its fitness score by stopping the reduction of training MSE when the validation MSE starts to go up.

Finally, for GetCommittee, the former validation data (1 through 1001 for input and 2 through 1002 for target) were used for complete post-evolution SCG training and the rest of the data (1003 through 1499 for input and 1004 through 1500 for the corresponding targets) were used for test results.

\section{Results}

Best evolved network and committee of networks after post-evolution training by GetCommittee provided the following results:

Best_Net_MSE_Train $=0.0052$
Best_Net_MSE_Test $=0.0054$

Committee_MSE_Train $=0.0052$

Committee_MSE_Test $=0.0054$

Please see figures 29 through 51 for more details.

\section{Connection maps:}

1- Connection maps of the original ancestor of the best-evolved network are 


$$
\begin{gathered}
\text { input_connect }=\left[\begin{array}{l}
1 \\
1
\end{array}\right] \\
\text { layer_connect }=\left[\begin{array}{ll}
1 & 0 \\
1 & 0
\end{array}\right] \\
\text { output_connect }=\left[\begin{array}{l}
0 \\
1
\end{array}\right]
\end{gathered}
$$

2- Connection maps of the best-evolved network after 15 generations are

$$
\begin{gathered}
\text { input_connect }=\left[\begin{array}{l}
1 \\
1
\end{array}\right] \\
\text { layer_connect }=\left[\begin{array}{ll}
0 & 0 \\
1 & 0
\end{array}\right] \\
\text { output_connect }=\left[\begin{array}{l}
0 \\
1
\end{array}\right]
\end{gathered}
$$

General descriptors and strategy parameters:

1-The original ancestor of the best-evolved network:

size $($ total branches $)=22$

prune_threshold $=0.0467$

prune_threshold_SD $=0.0026$

node_mutation_SD $=0.0076$

connection_mutation_SD $=0.1834$

delay_mutation_SD $=0.6959$

Darwinian mutation' standard deviation (for Dar_inp_SD and Dar_lay_SD) $=0.0235$ 
Darwinian mutation' mean (for Dar_inp_SD and Dar_lay_SD) $=0.0413$

Connection weights' standard deviation $=0.5257$

Connection weights' mean $=-0.1525$

Training/validation time (mean of multiple starts) $=22.5430 \mathrm{sec}$

2- The best evolved network, after 203 generations:

size $($ total branches $)=16$

prune_threshold $=0.0037$

prune_threshold_SD $=0.0005307$

node_mutation_SD $=0.0101$

connection_mutation_SD $=0.0001917$

delay_mutation_SD $=0.0012$

Darwinian mutation' standard deviation (for Dar_inp_SD and Dar_lay_SD) $=0.0026$

Darwinian mutation' mean (for Dar_inp_SD and Dar_lay_SD) $=0.0014$

Connection weights' standard deviation $=0.5721$

Connection weights' mean $=-0.1326$

Training/validation time (mean of multiple starts) $=6.2690 \mathrm{sec}$

Weights:

1- Connection weights, original ancestor of the best-evolved network: 


$$
\begin{gathered}
\text { input_weights }=\left[\begin{array}{l}
\overrightarrow{i w}_{11} \\
\overrightarrow{i w}_{21}
\end{array}\right] \\
\text { layer_weights }=\left[\begin{array}{ll}
\overrightarrow{l w}_{11} & {[]} \\
\overrightarrow{l w}_{21} & {[}
\end{array}\right]
\end{gathered}
$$

Starting with the following vector elements:

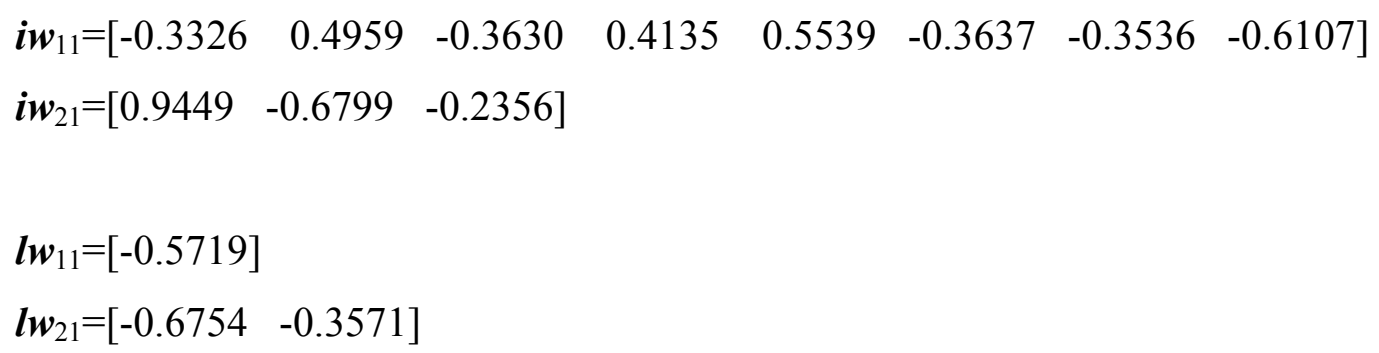

2- Connection weights, best evolved network after 203 generations:

$$
\begin{gathered}
\text { input_weights }=\left[\begin{array}{l}
\overrightarrow{i w}_{11} \\
\overrightarrow{i w}_{21}
\end{array}\right] \\
\text { layer_weights }=\left[\begin{array}{ll}
{[]} & {[]} \\
\overrightarrow{l w}_{21} & {[]}
\end{array}\right]
\end{gathered}
$$

with the following vector element:

$$
\begin{aligned}
& \boldsymbol{i} \boldsymbol{w}_{11}=\left[\begin{array}{llllll}
-0.4787 & 0.8929 & -0.8379 & -0.6025 & -0.6077 & 0.7196
\end{array}\right] \\
& \boldsymbol{i} \boldsymbol{w}_{21}=\left[\begin{array}{llll}
1.0053 & -0.7330 & 0.0845 & -0.1311
\end{array}\right] \\
& \boldsymbol{l} \boldsymbol{w}_{21}=\left[\begin{array}{llllll}
-0.6401 & 0.1007 & -0.3075 & -0.2656 & -0.1229 & -0.1971
\end{array}\right]
\end{aligned}
$$




\section{Weight Evolution}

1- Standard deviation matrices of weight perturbation, original ancestor of the bestevolved network:

$$
\begin{gathered}
\text { Dar_inp_SD }=\left[\begin{array}{l}
\overrightarrow{d i S D}_{11} \\
\overrightarrow{d i S D}_{21}
\end{array}\right] \\
D a r_{2} l a y_{-} S D=\left[\begin{array}{ll}
\overrightarrow{d l S D}_{11} & {[]} \\
\overrightarrow{d l S D}_{21} & {[]}
\end{array}\right]
\end{gathered}
$$

Starting with the following vector elements:

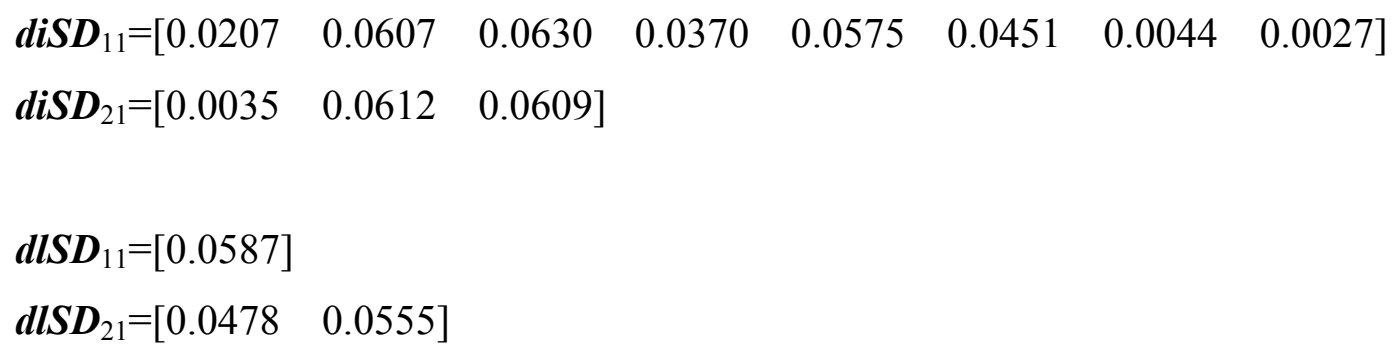

2- Standard deviation matrices of weight perturbation, best-evolved network after 203 generations:

$$
\begin{aligned}
& D a r_{-} i n p_{-} S D=\left[\begin{array}{l}
\overrightarrow{d i S D}_{11} \\
\overrightarrow{d i S D}_{21}
\end{array}\right]
\end{aligned}
$$

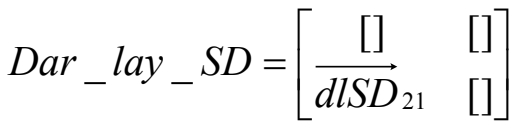

with the following vector elements:

$$
\operatorname{diSD}_{11}=\left[\begin{array}{llllll}
0.0012 & 0.0016 & 0.0091 & 0.0064 & 0.0000 & 0.0000
\end{array}\right]
$$


$\operatorname{diSD}_{21}=\left[\begin{array}{llll}0.0000032 & 0.0000889 & 0.0008957 & 0.0008392\end{array}\right]$

$\boldsymbol{d l S D _ { 2 1 }}=\left[\begin{array}{llllll}0.0005 & 0.0001 & 0.0004 & 0.0000 & 0.0000 & 0.0015\end{array}\right]$

Delays

1- Branch delays matrices of the original ancestor of the best-evolved network:

$$
\begin{gathered}
\text { input_delay }=\left[\begin{array}{l}
\overrightarrow{i d}_{11} \\
\overrightarrow{i d}_{21}
\end{array}\right] \\
\text { layer_delay }=\left[\begin{array}{ll}
\overrightarrow{l d}_{11} & {[]} \\
\overrightarrow{l d}_{21} & {[]}
\end{array}\right]
\end{gathered}
$$

Starting with the following vector elements:

$$
\begin{aligned}
& \boldsymbol{i d}_{11}=\left[\begin{array}{llllllll}
2 & 3 & 4 & 7 & 9 & 10 & 13 & 14
\end{array}\right] \\
& \boldsymbol{i d}_{21}=\left[\begin{array}{lll}
0 & 1 & 6
\end{array}\right] \\
& \boldsymbol{l \boldsymbol { d } _ { 1 1 }}=\left[\begin{array}{ll}
3
\end{array}\right] \\
& \boldsymbol{l \boldsymbol { d } _ { 2 1 }}=\left[\begin{array}{ll}
2 & 5
\end{array}\right]
\end{aligned}
$$

2- Branch delays, best evolved network after 203 generations:

$$
\begin{gathered}
\text { input_delay }=\left[\begin{array}{l}
\overrightarrow{i d}_{11} \\
\overrightarrow{i d}_{21}
\end{array}\right] \\
\text { layer_delay }=\left[\begin{array}{ll}
{[]} & {[]} \\
\overrightarrow{l d}_{21} & {[]}
\end{array}\right]
\end{gathered}
$$


with the following vector elements:

$\boldsymbol{i d}_{11}=\left[\begin{array}{llllll}2 & 3 & 4 & 13 & 14 & 21\end{array}\right]$
$\boldsymbol{i d}_{21}=\left[\begin{array}{lllll}0 & 1 & 10 & 15\end{array}\right]$
$\boldsymbol{l d}_{21}=\left[\begin{array}{llllll}2 & 4 & 5 & 9 & 18 & 34\end{array}\right]$

The following figure shows the best evolved network.

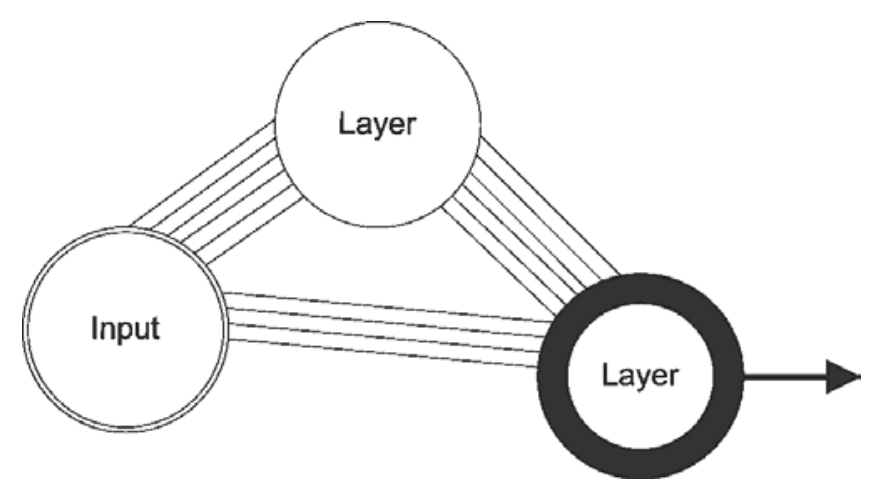

Figure 28 Best evolved network for MG17 six-step prediction. Each line represents a delayed synaptic connection between one input and two layer nodes. 


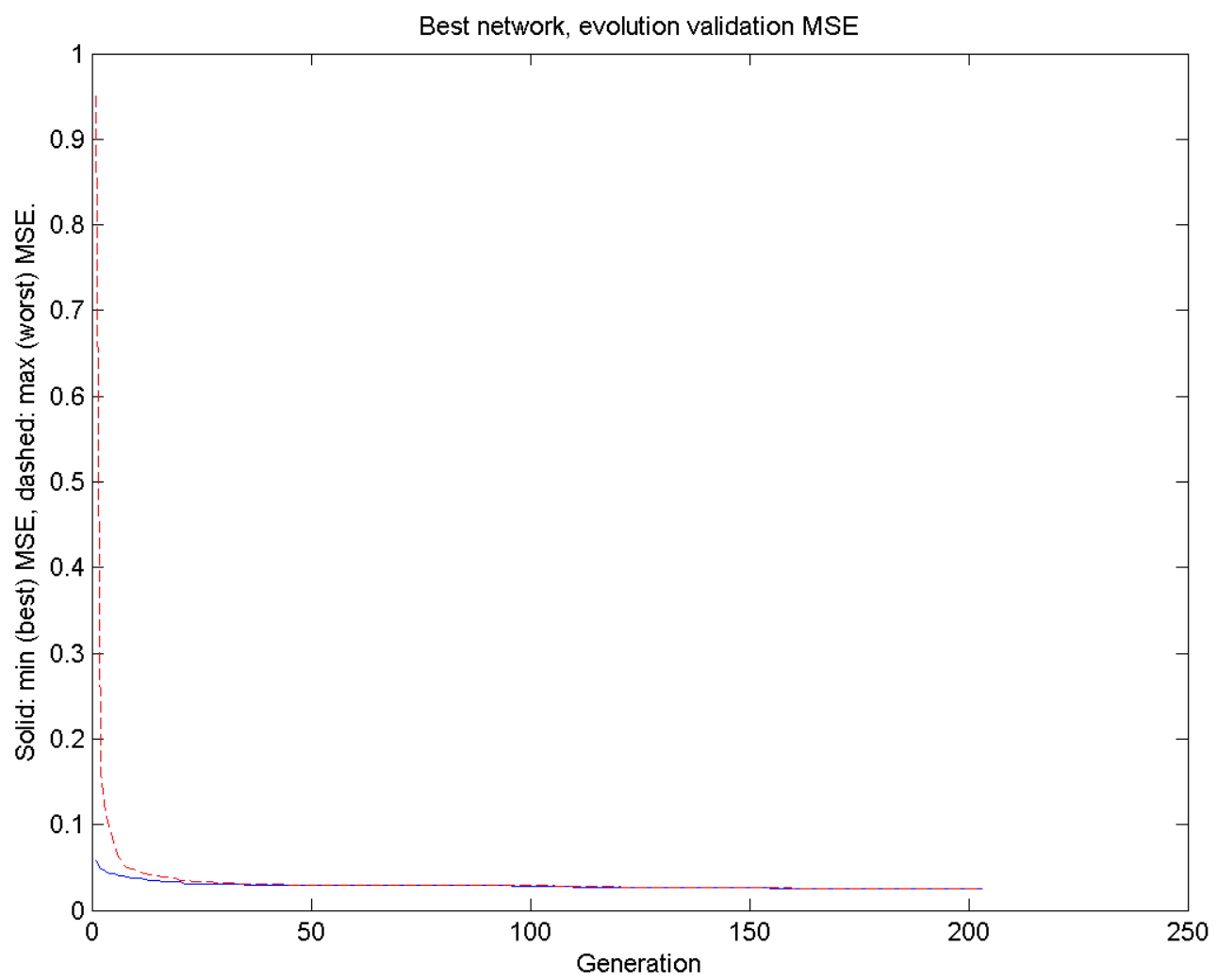

Figure 29 MSE of evolving networks.

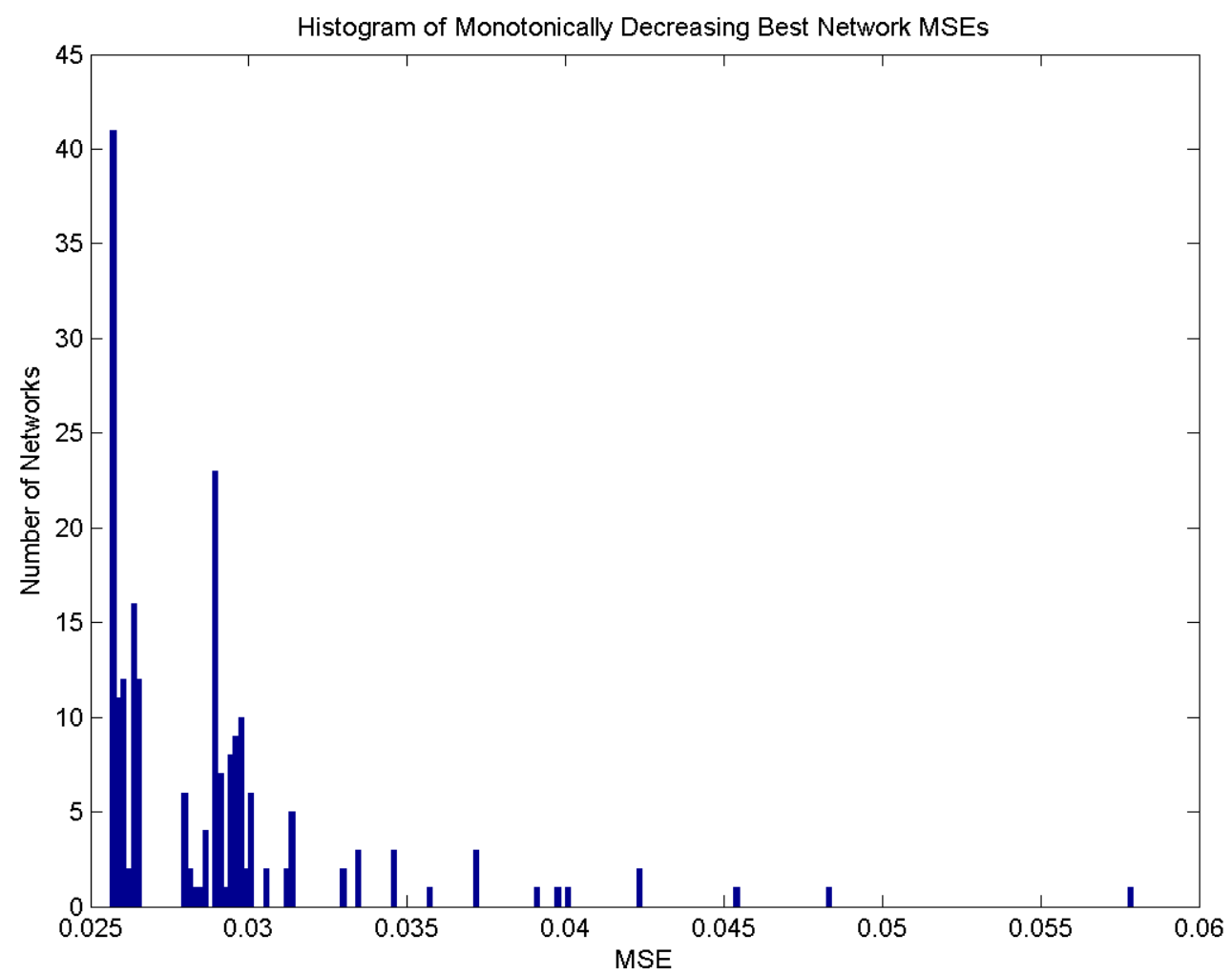

Figure 30 Histogram of the MSEs of the best networks through 203 generations. 


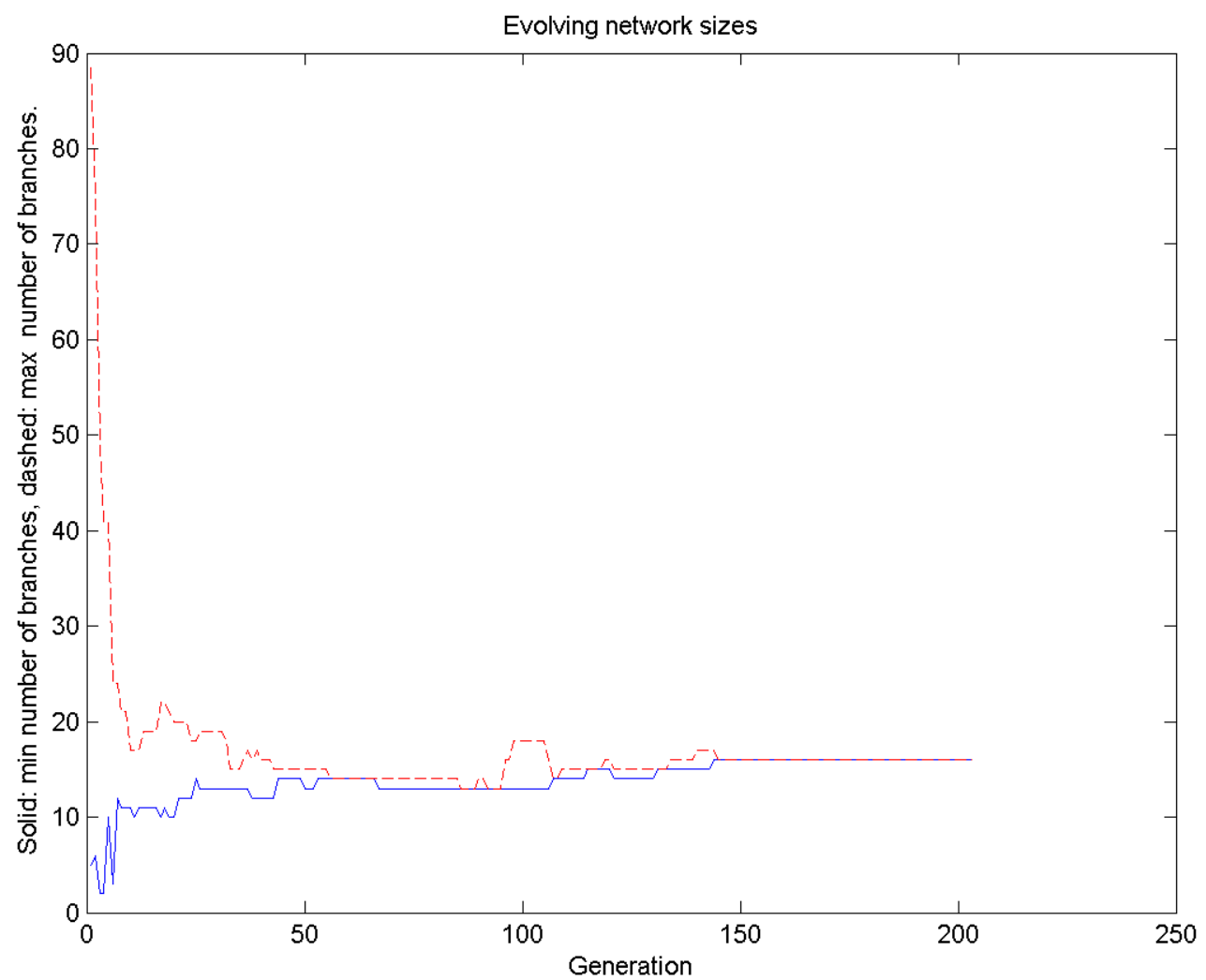

Figure 31 Size of evolving networks.

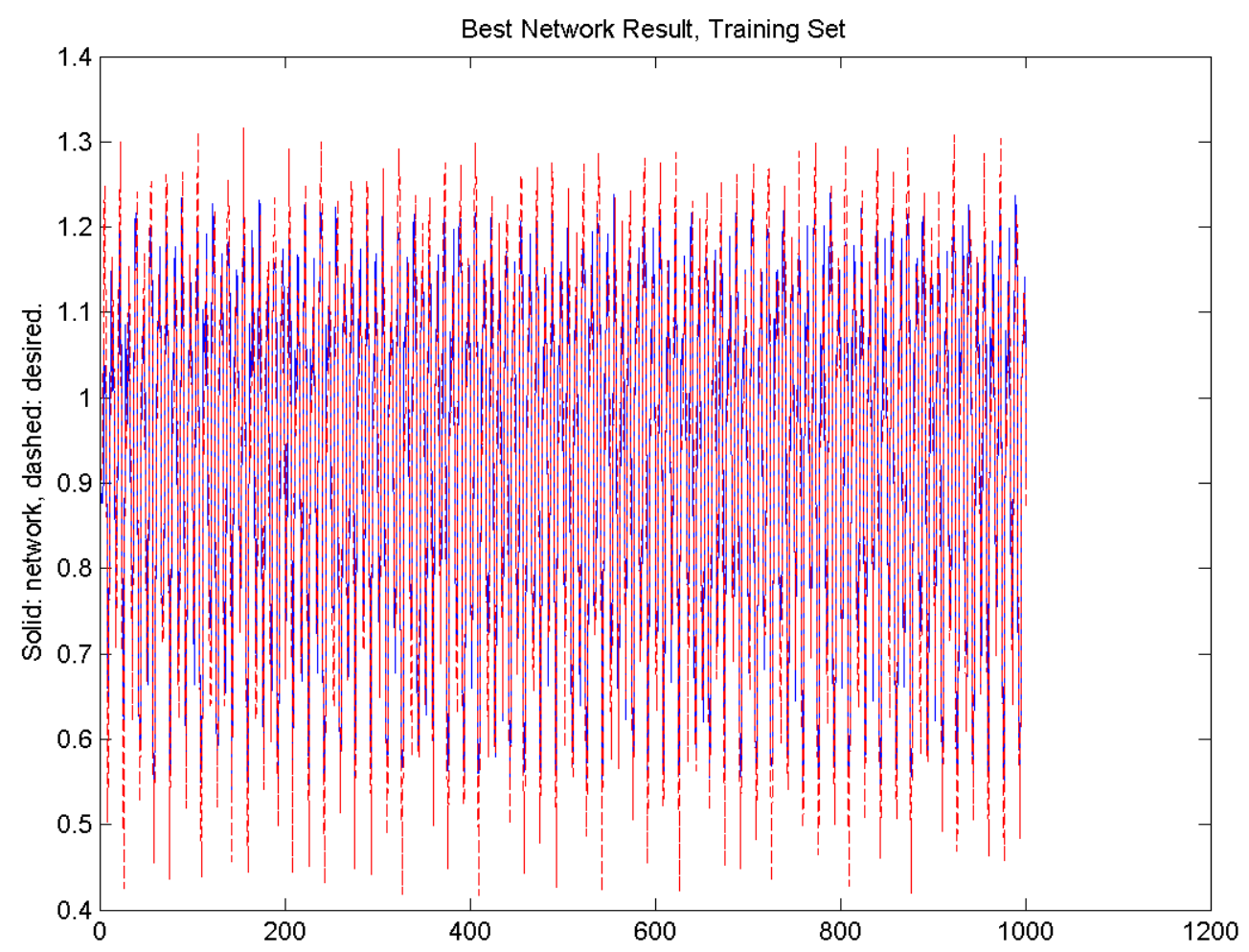

Figure 32 Training data, best evolved network. 


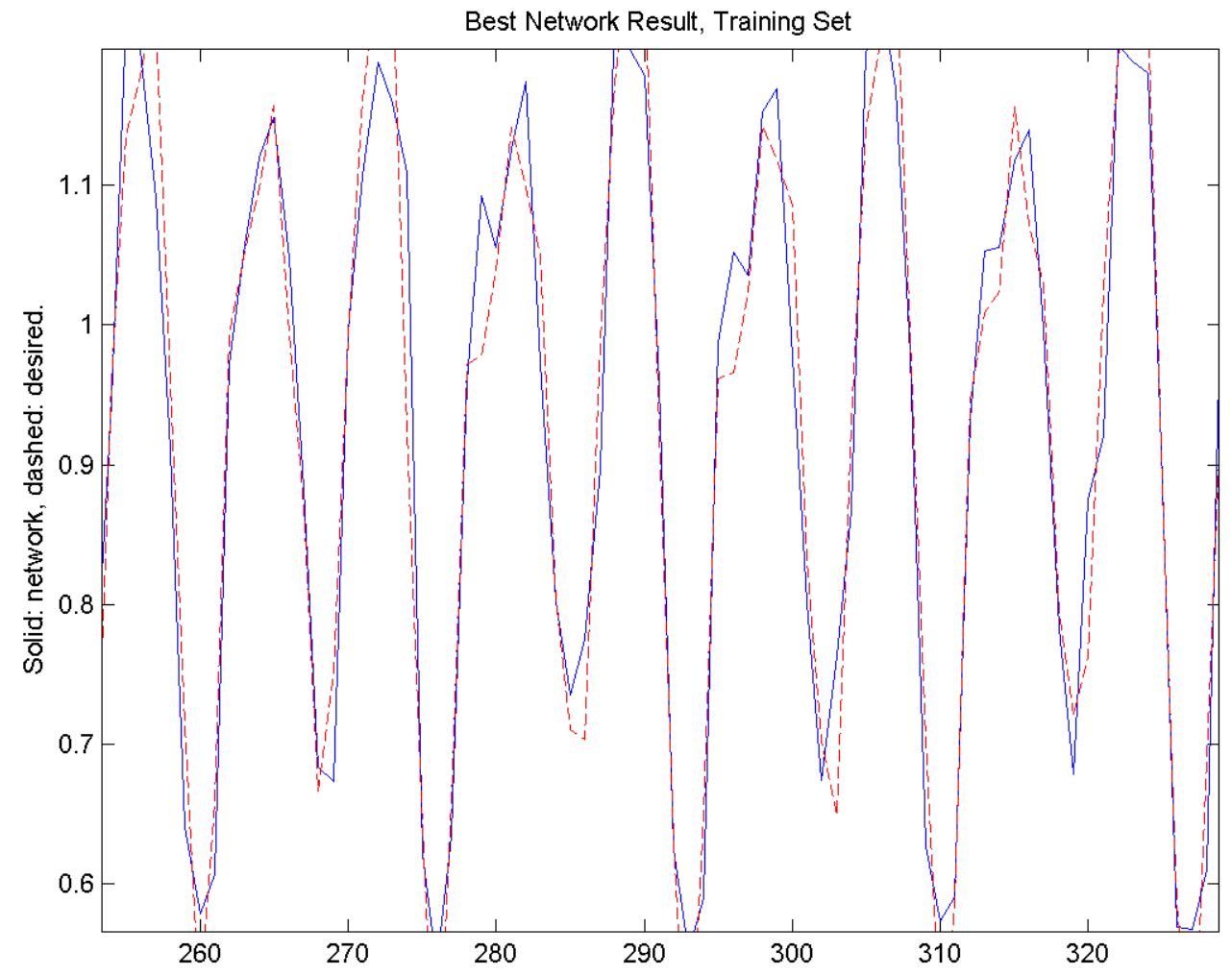

Figure 33 Training data, magnified section, best evolved network.

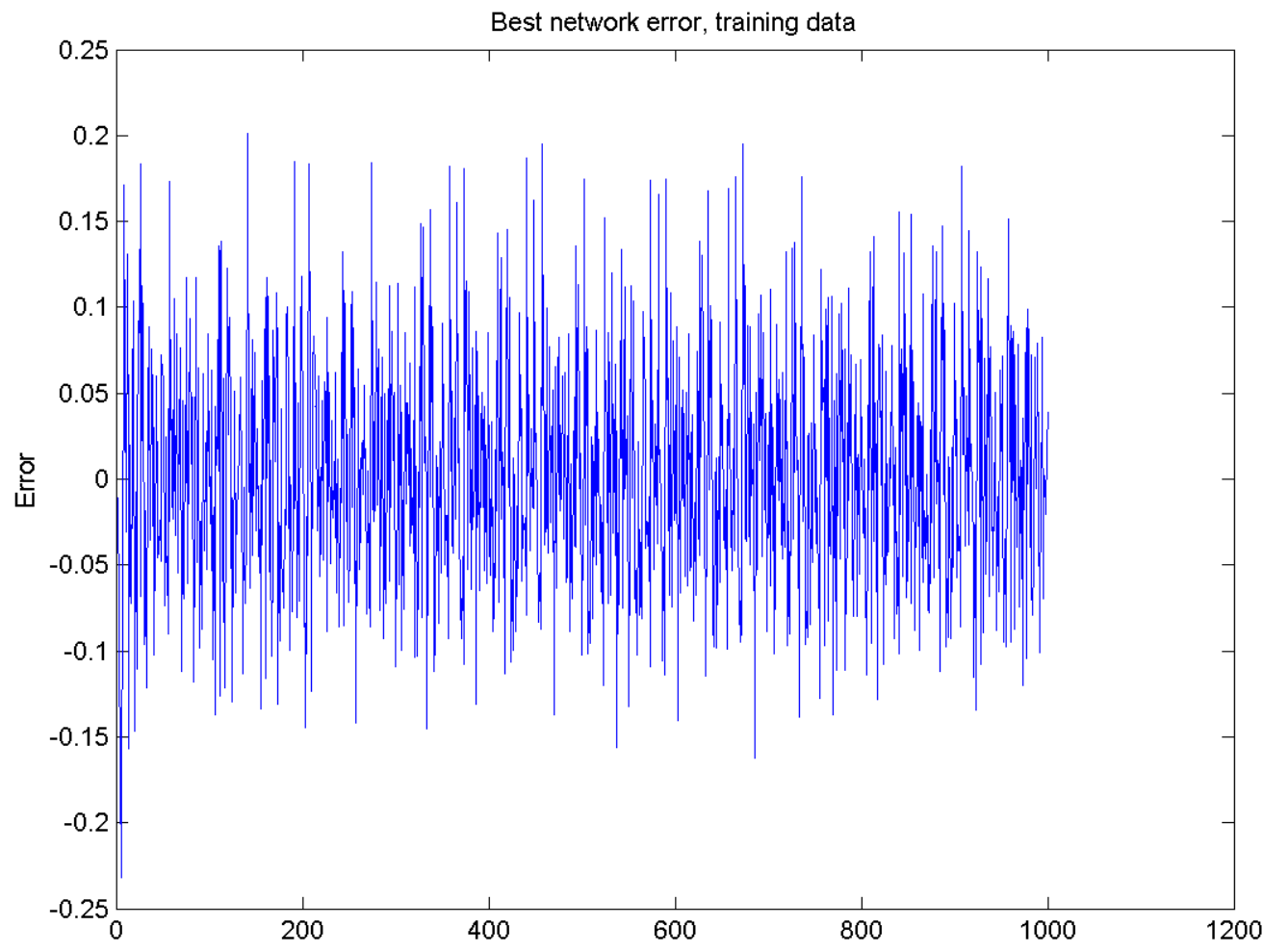

Figure 34 Best evolved network, training error. 


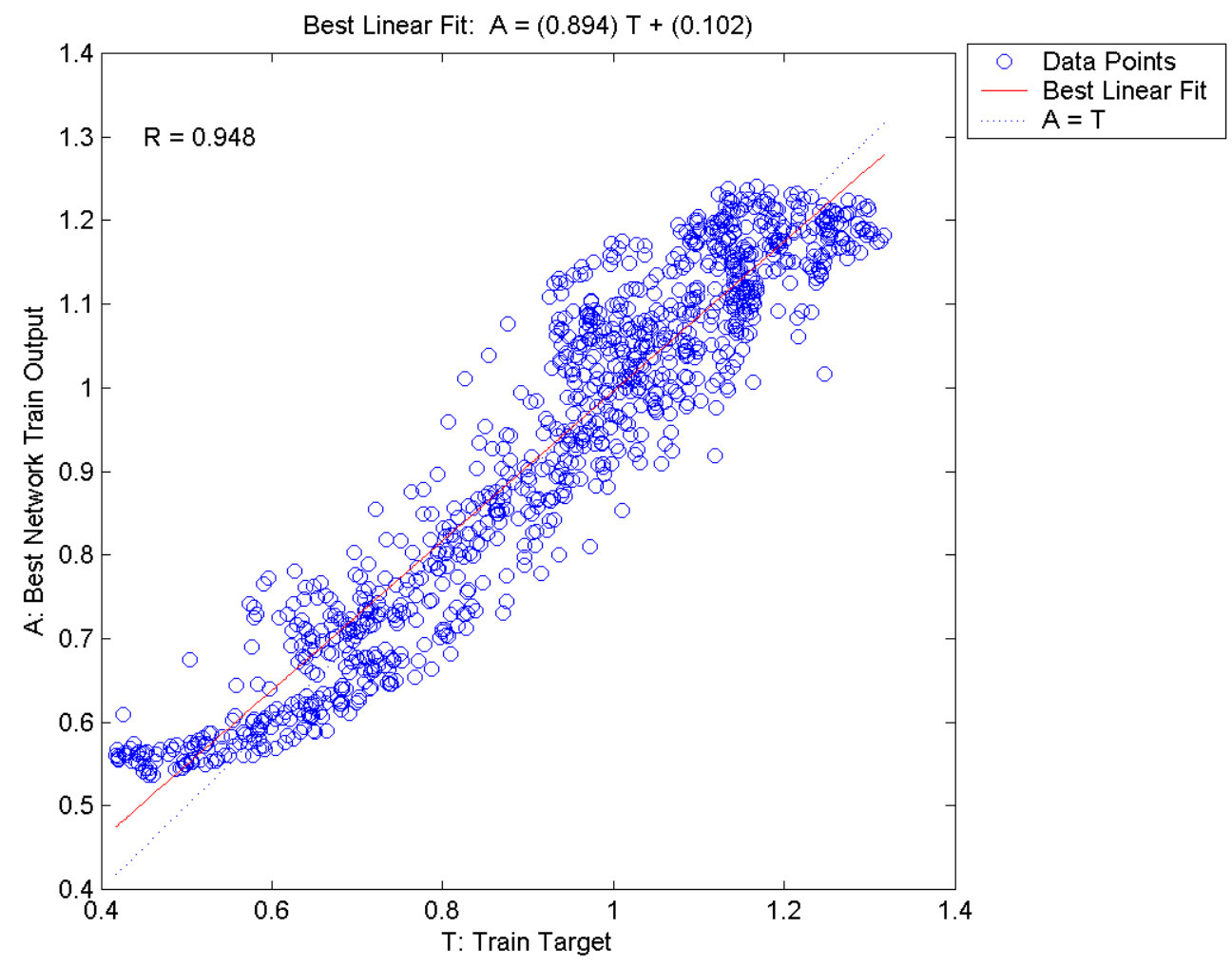

Figure 35 Best evolved network: training performance correlation.

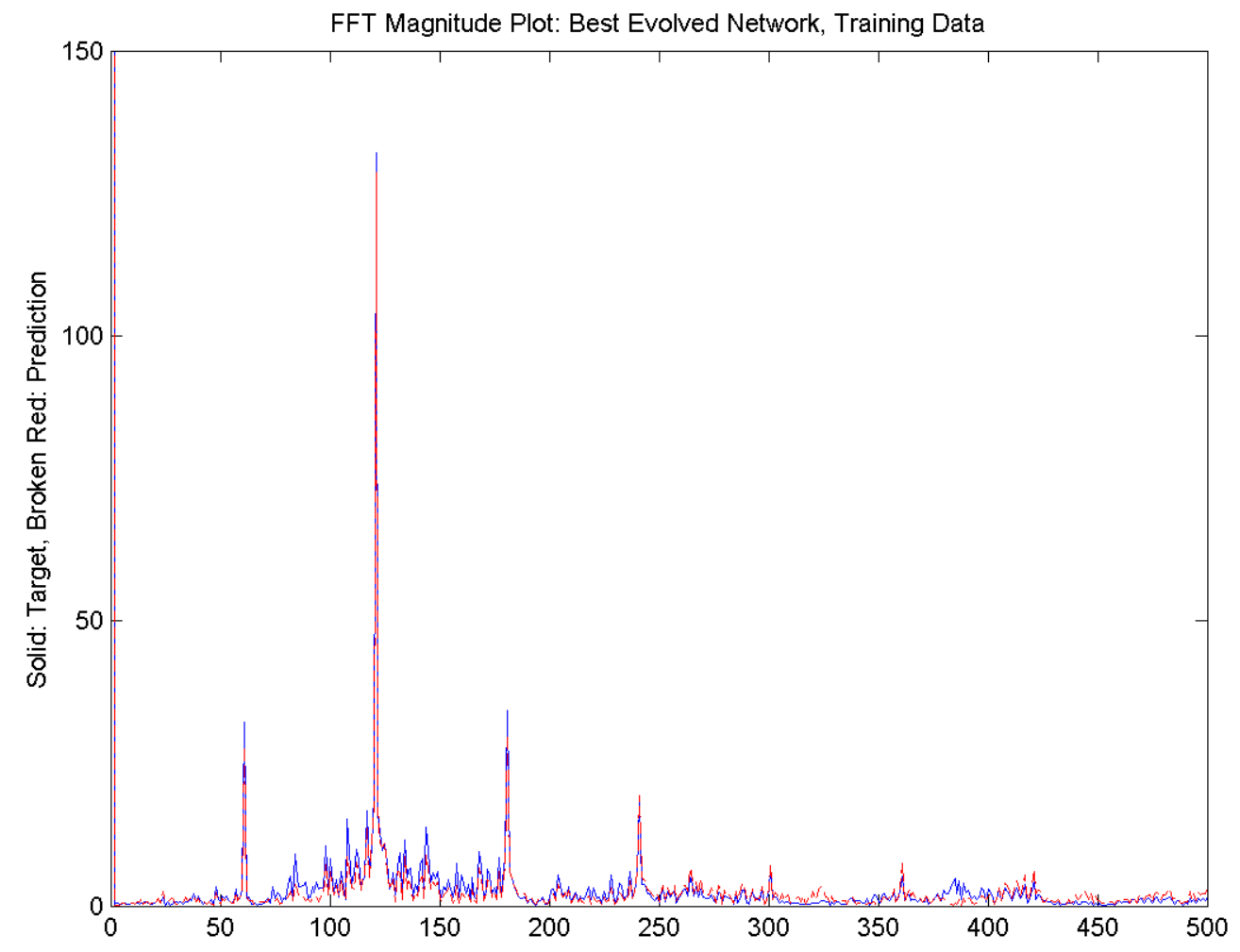

Figure 36 Best evolved network, training data Fourier transform magnitude plots. 


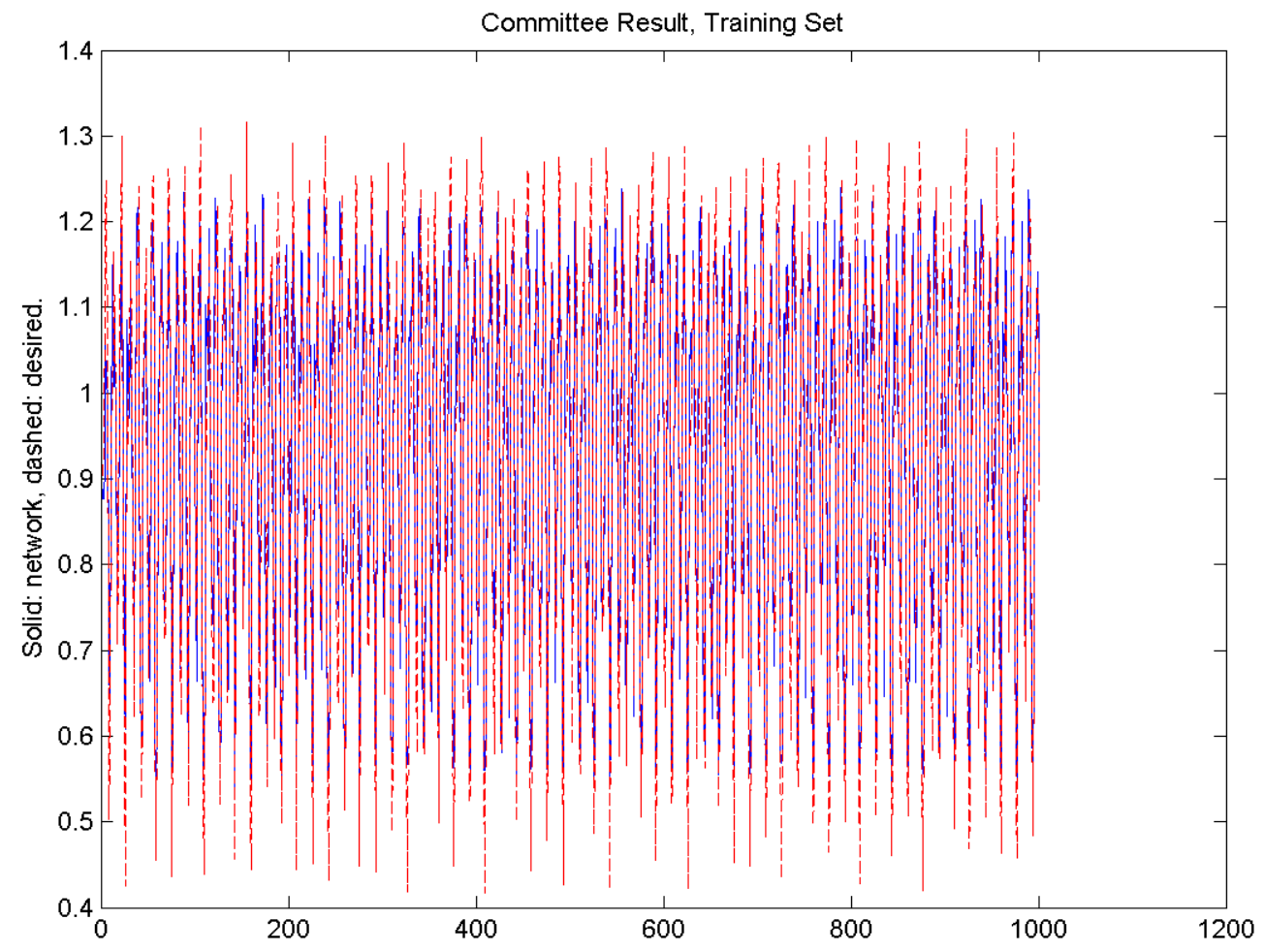

Figure 37 Training data, committee of last generation networks.

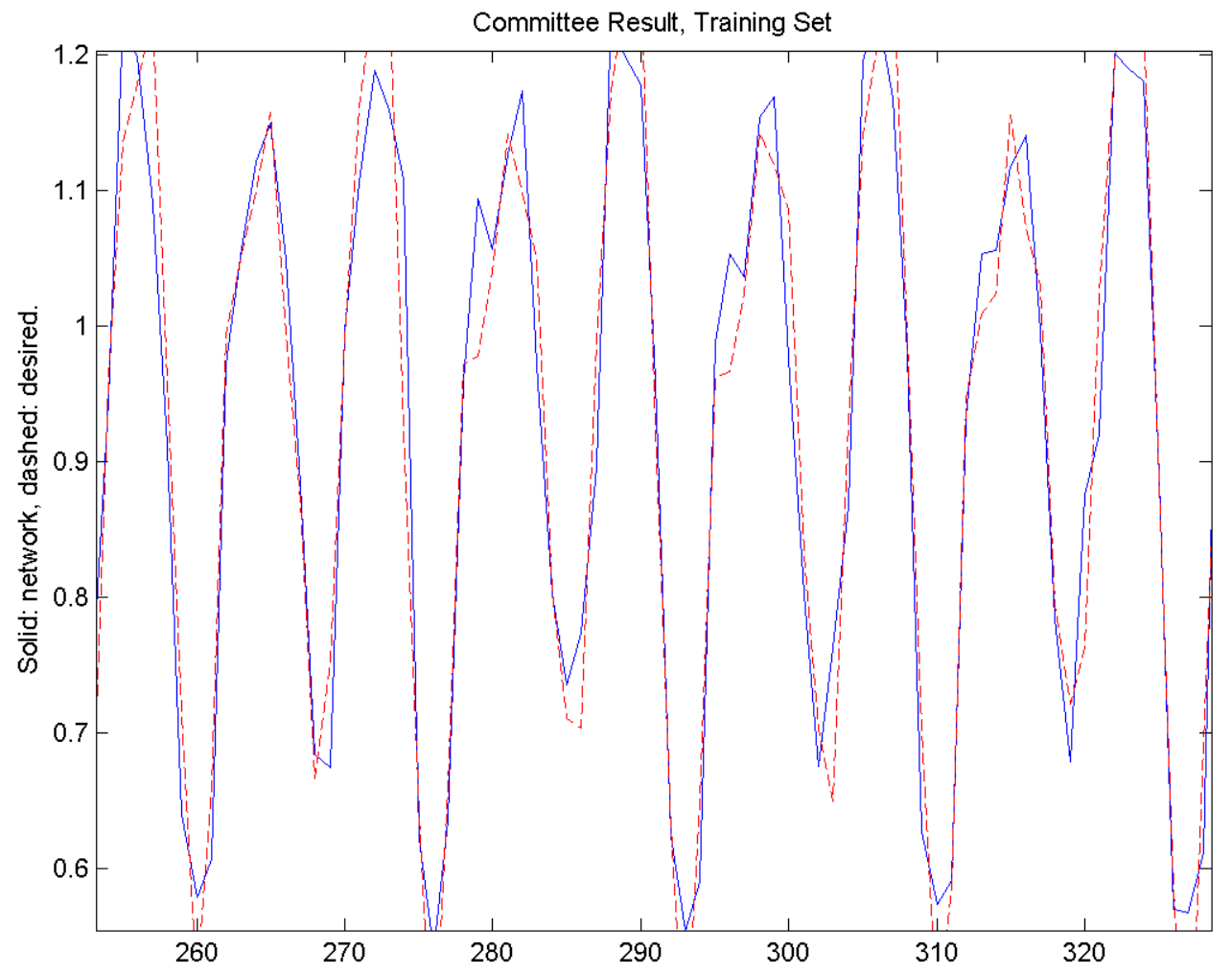

Figure 38 Training data, magnified section for network committee. 


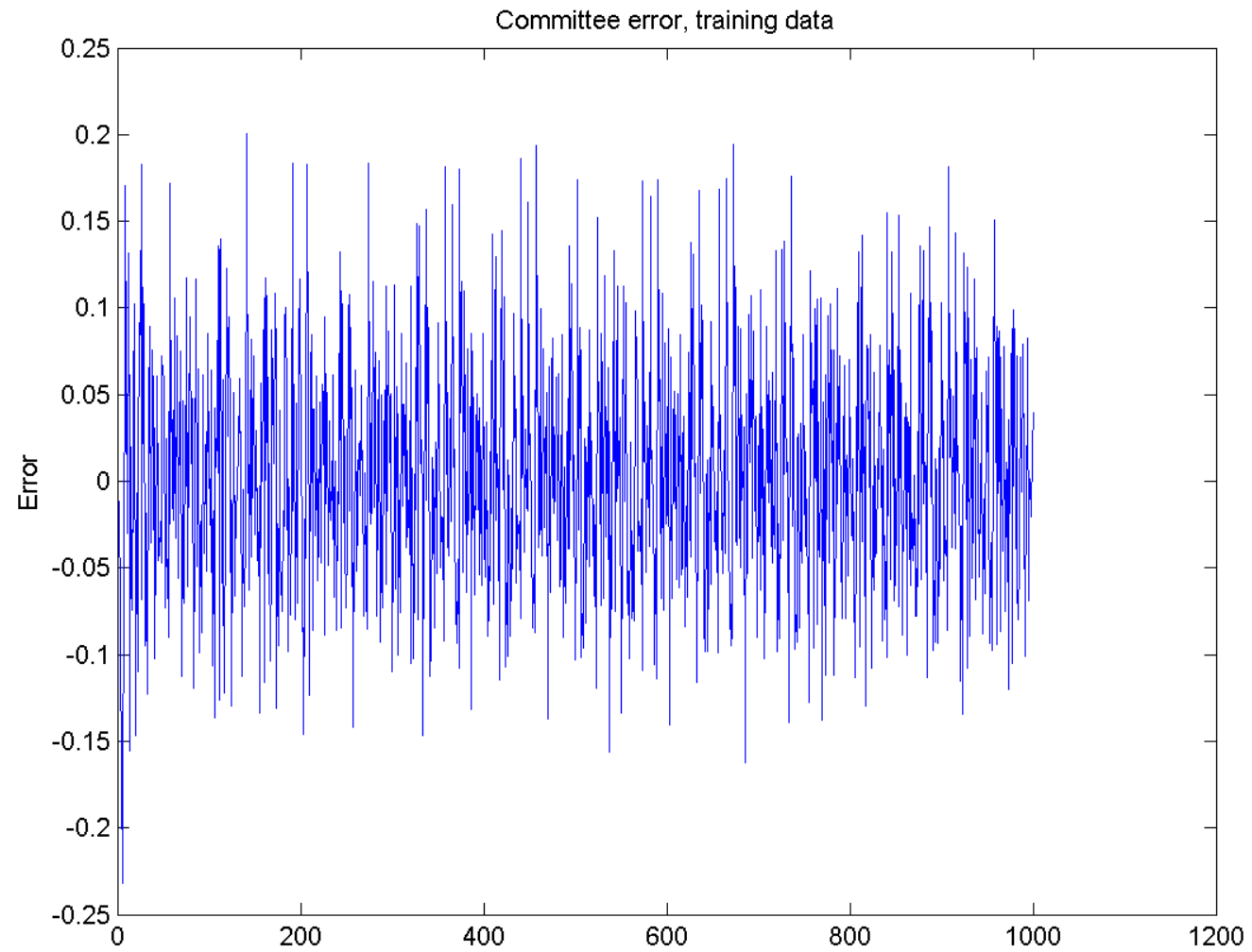

Figure 39 Network committee, training error.

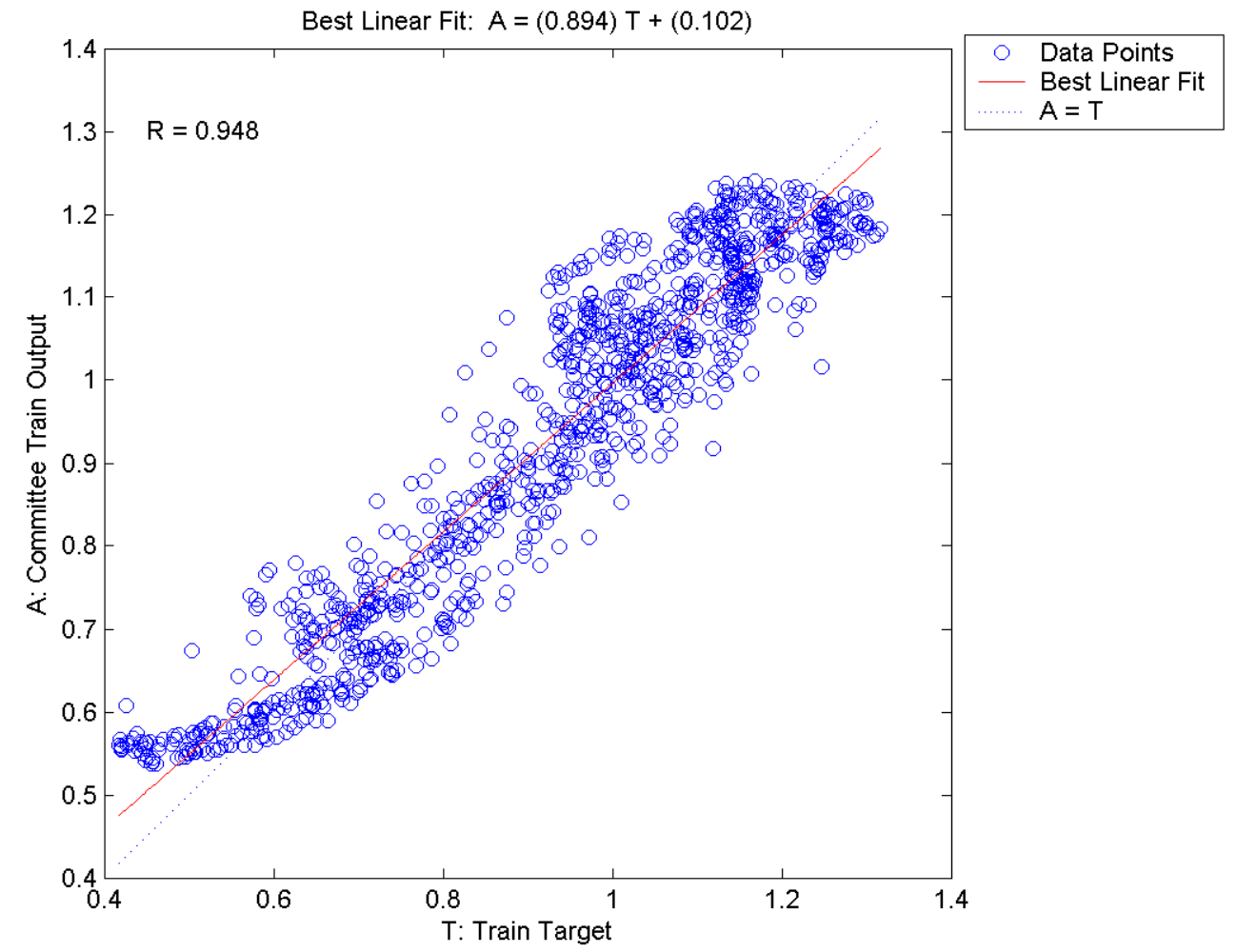

Figure 40 Network committee: training performance correlation. 


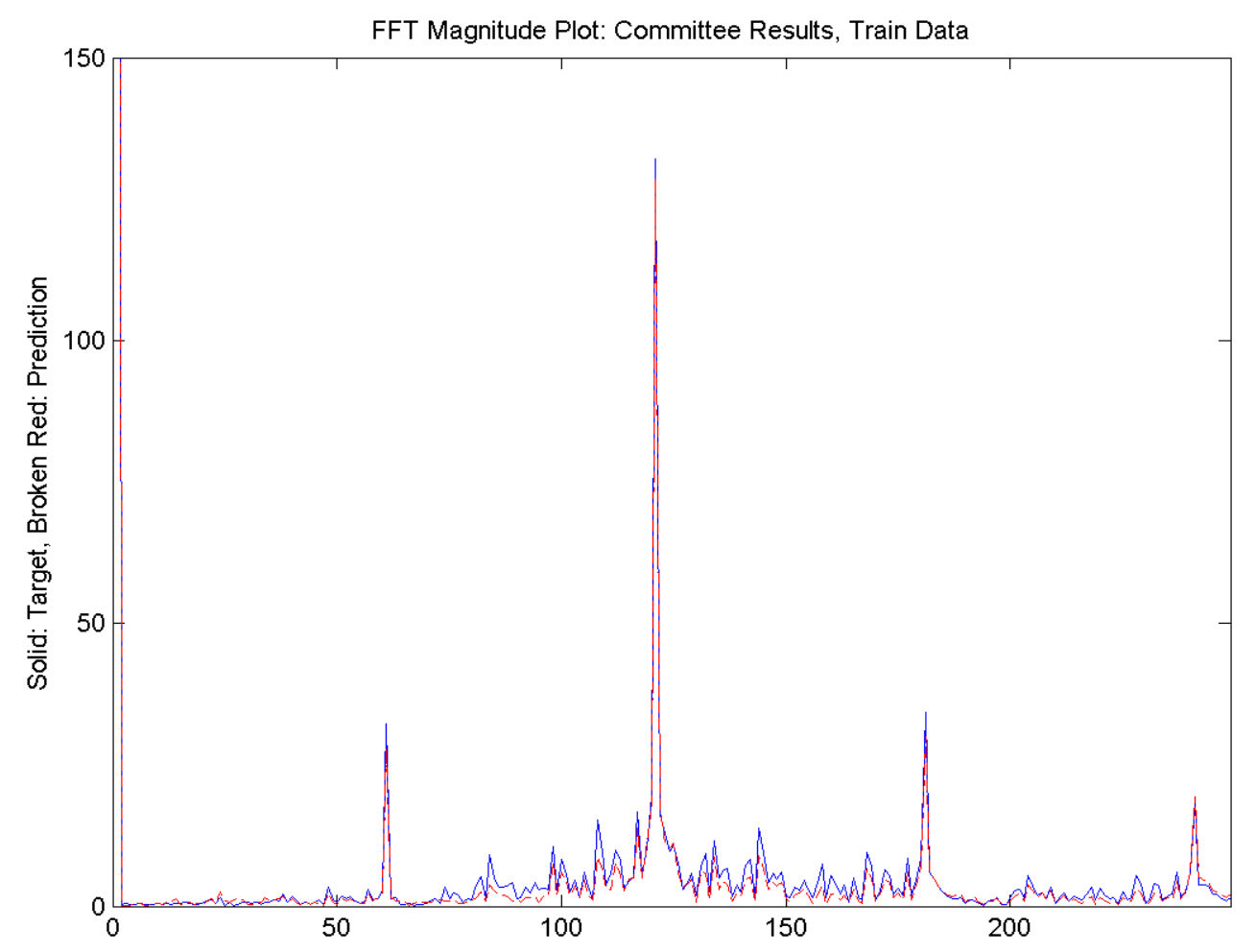

Figure 41 Network committee, training data Fourier transform magnitude plots.

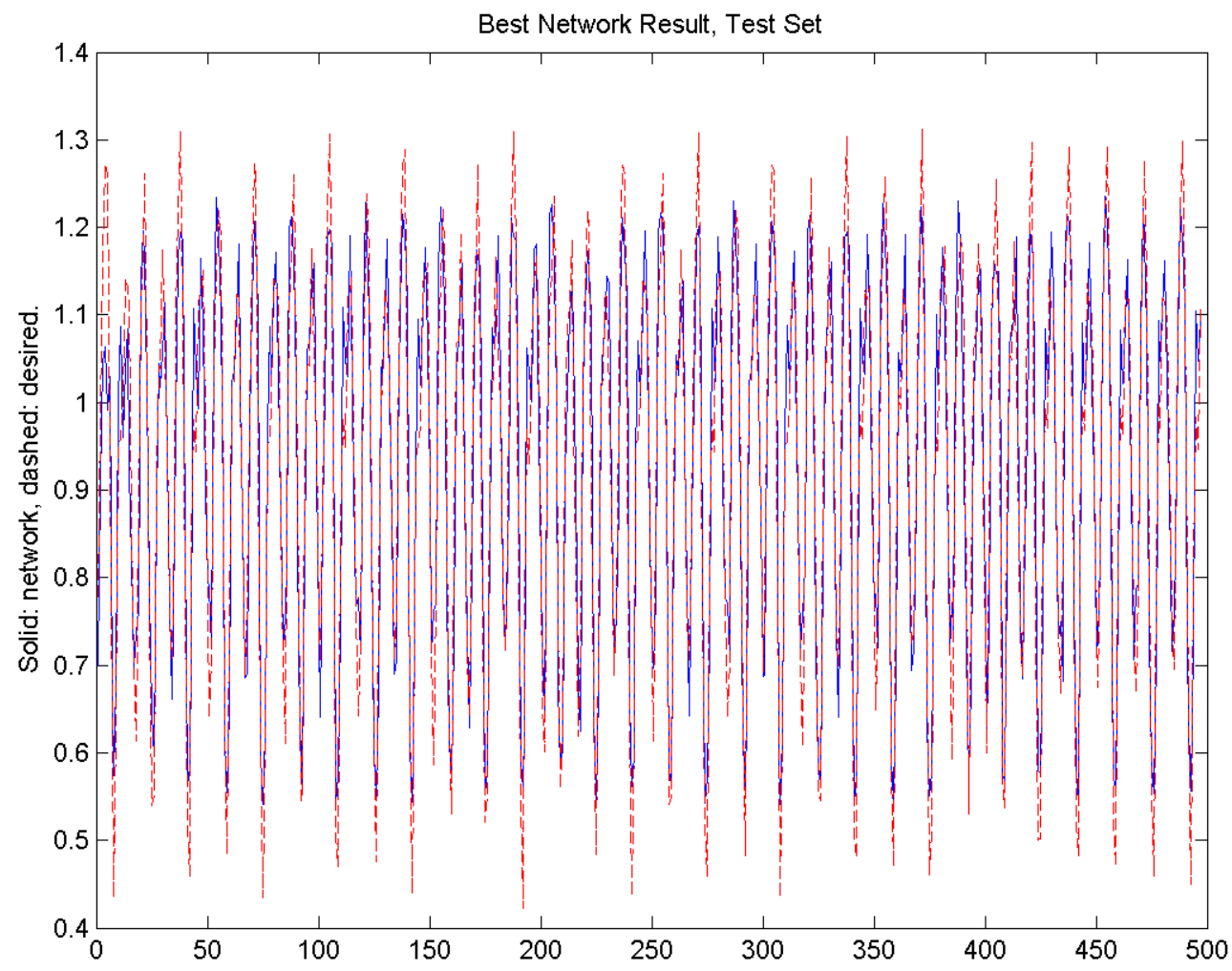

Figure 42 Test data, best evolved network. 


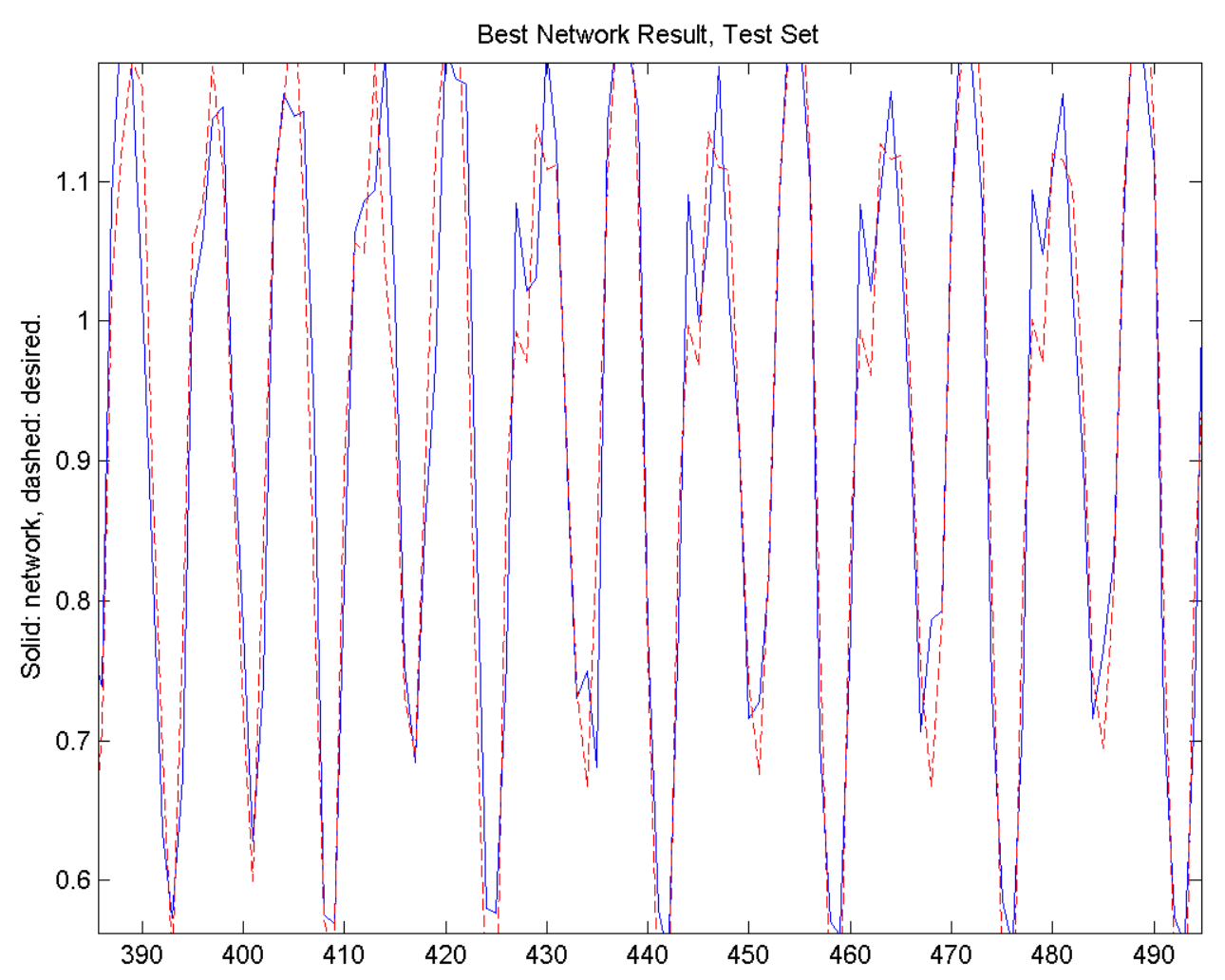

Figure 43 Test set performance, magnified.

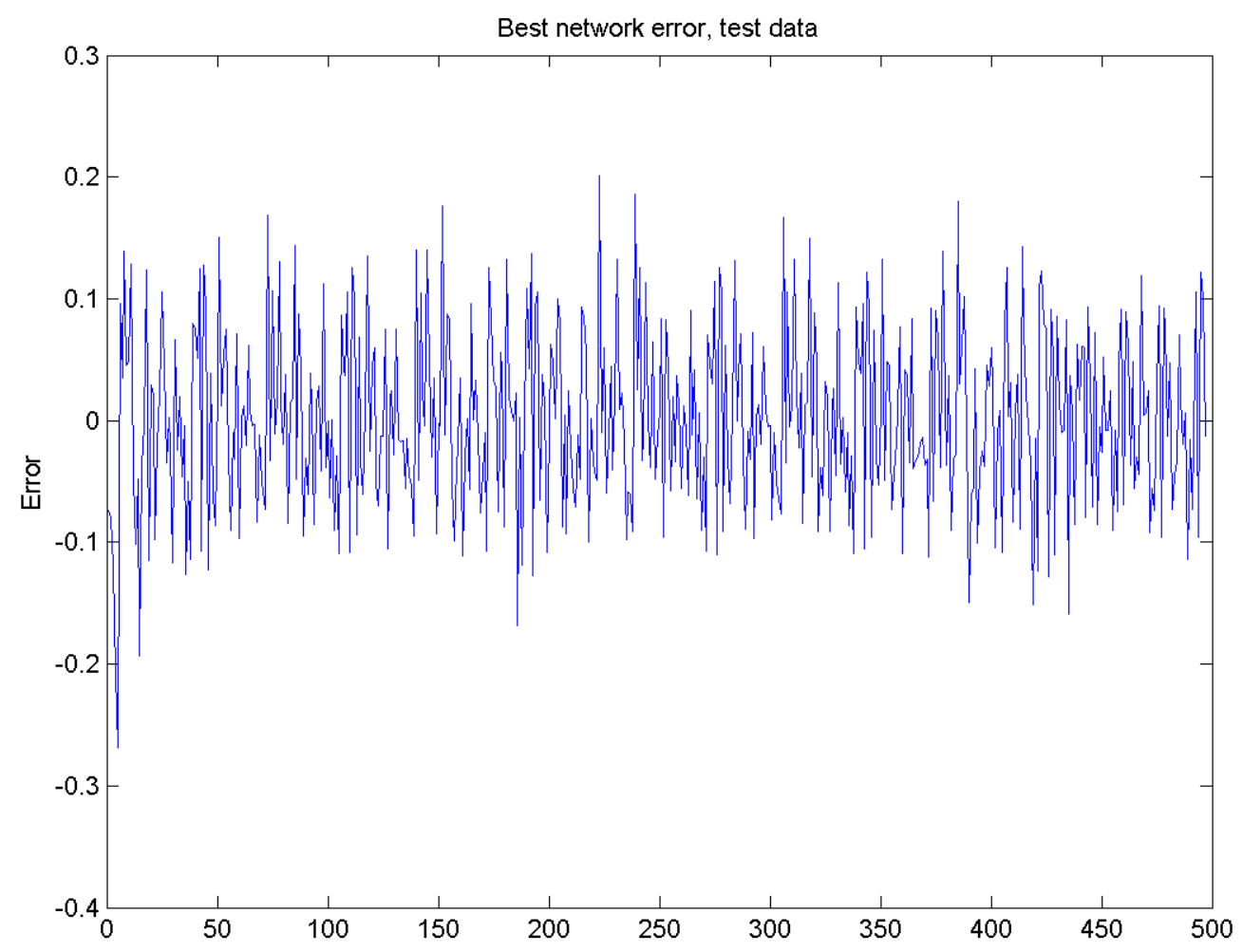

Figure 44 Best network, test data error. 


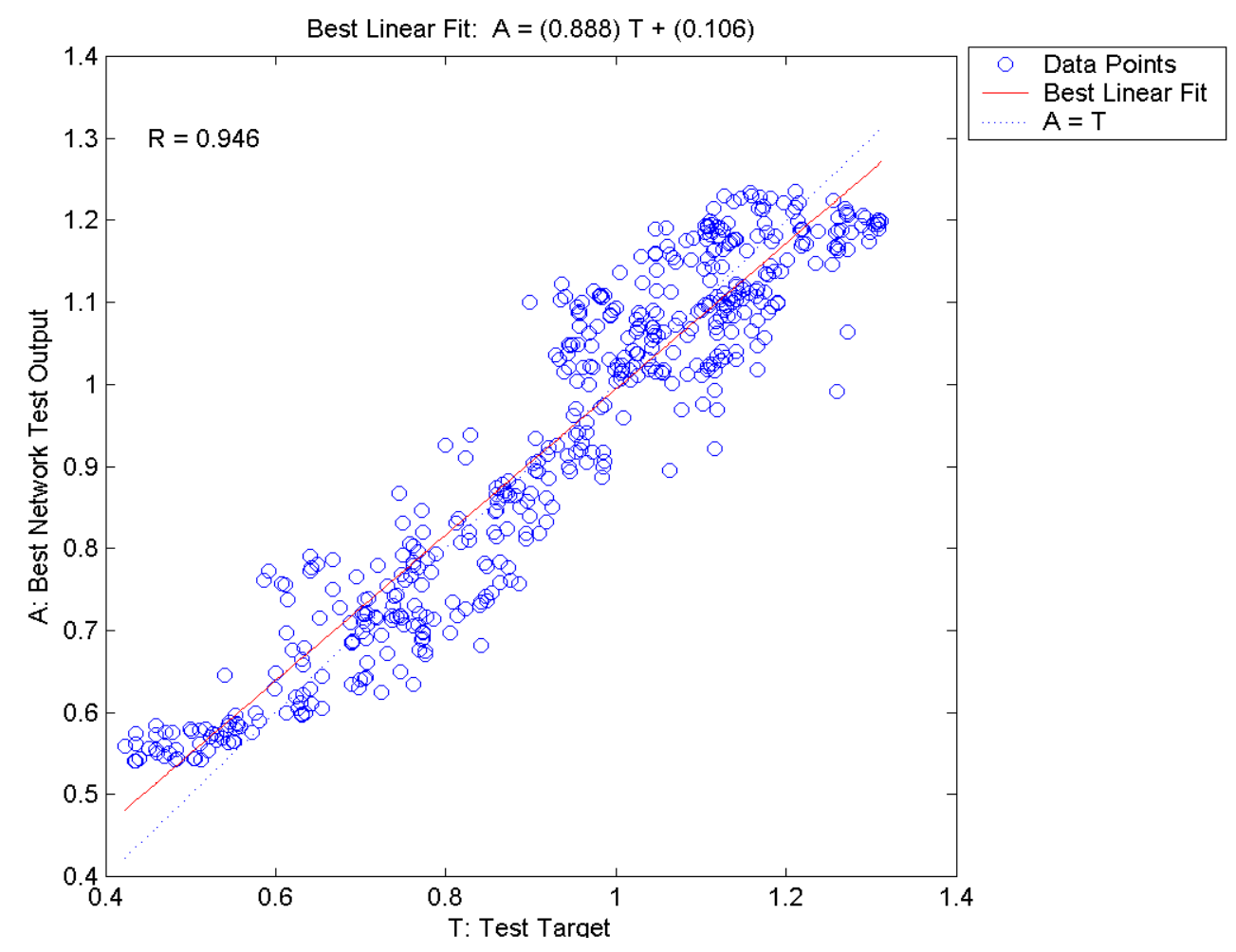

Figure 45 Best evolved network, test set performance correlation.

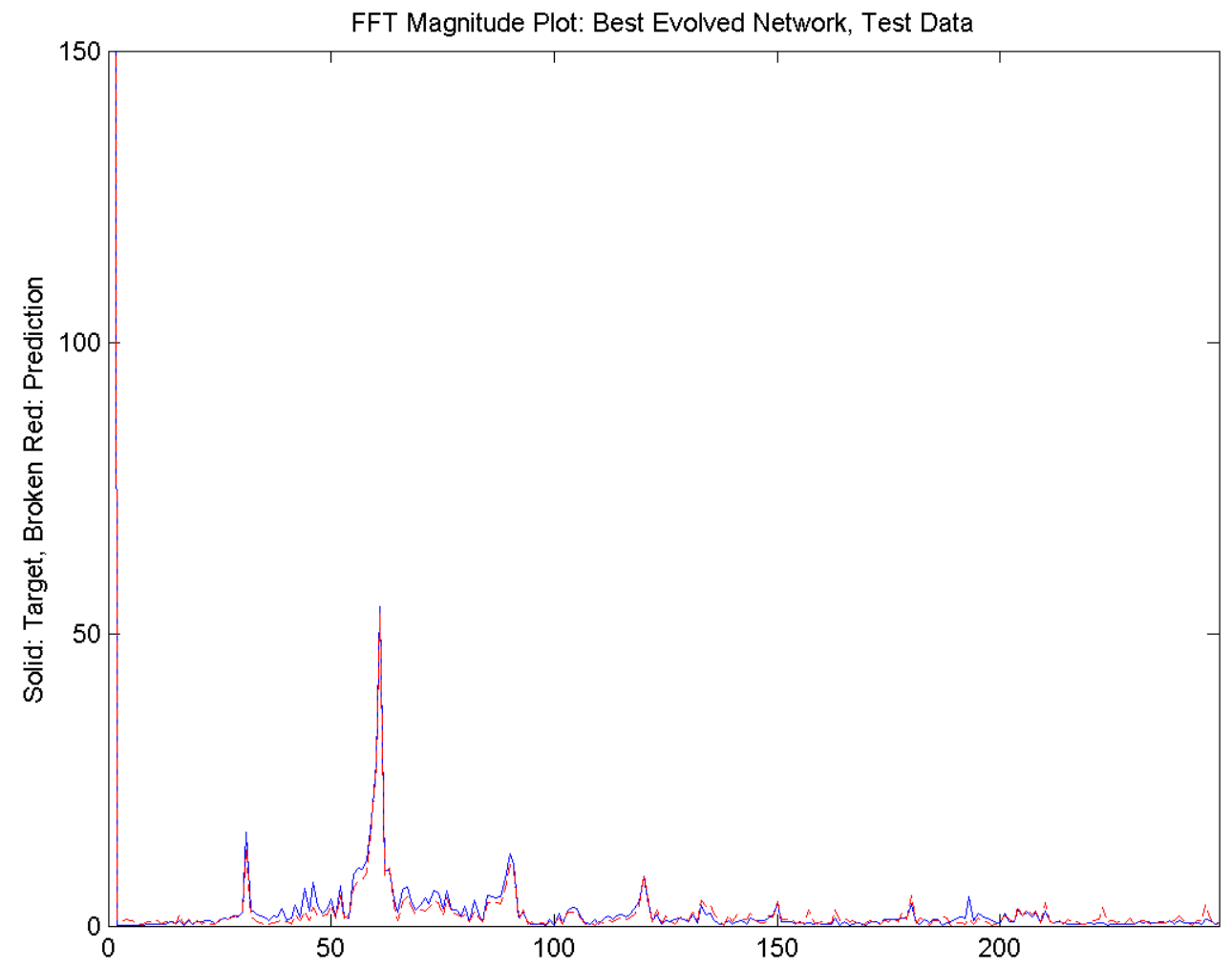

Figure 46 Best evolved network, test data Fourier transform magnitude plots. 


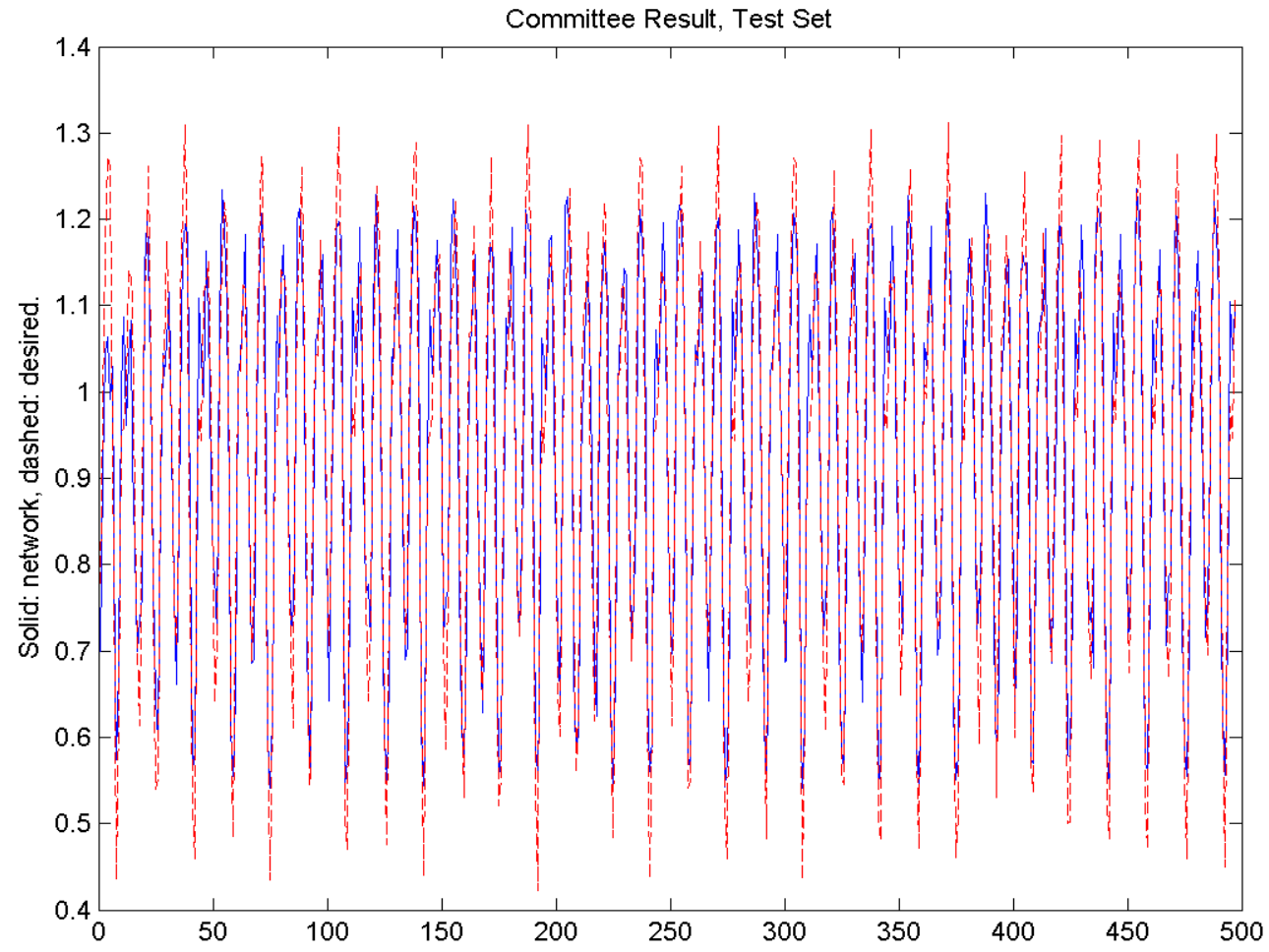

Figure 47 Test data, committee of last generation networks.

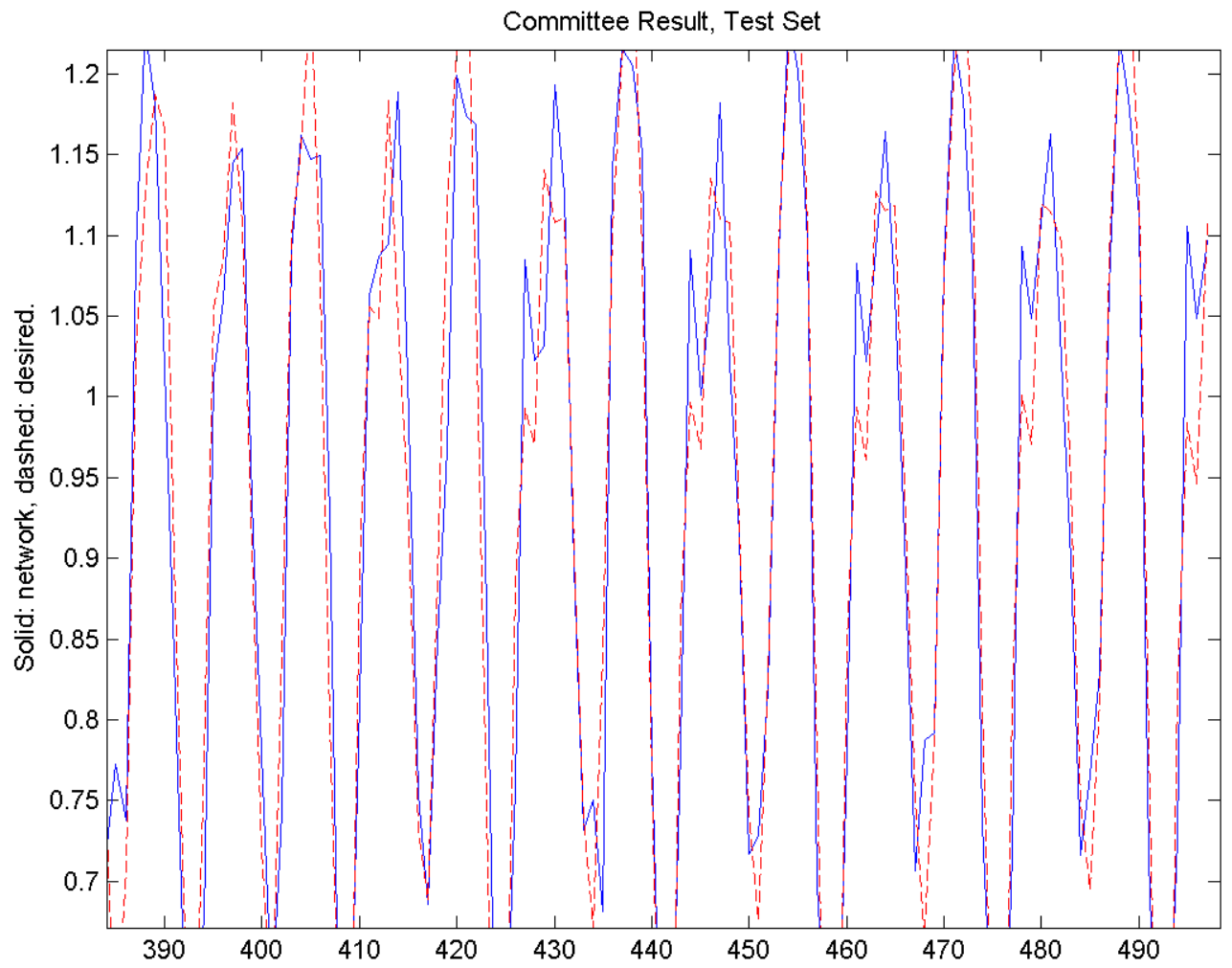

Figure 48 Test set performance, magnified section for network committee. 


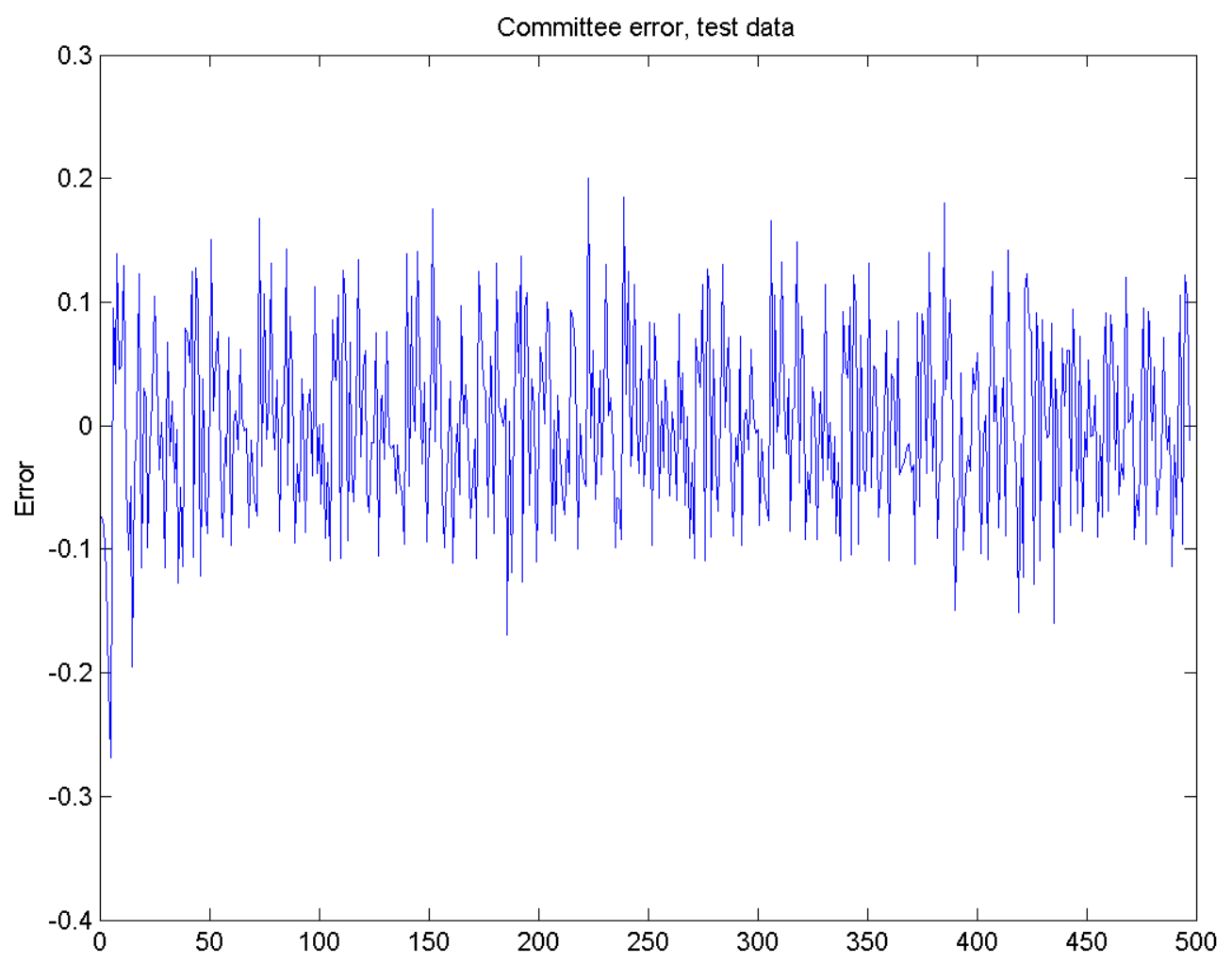

Figure 49 Network committee, test data error.

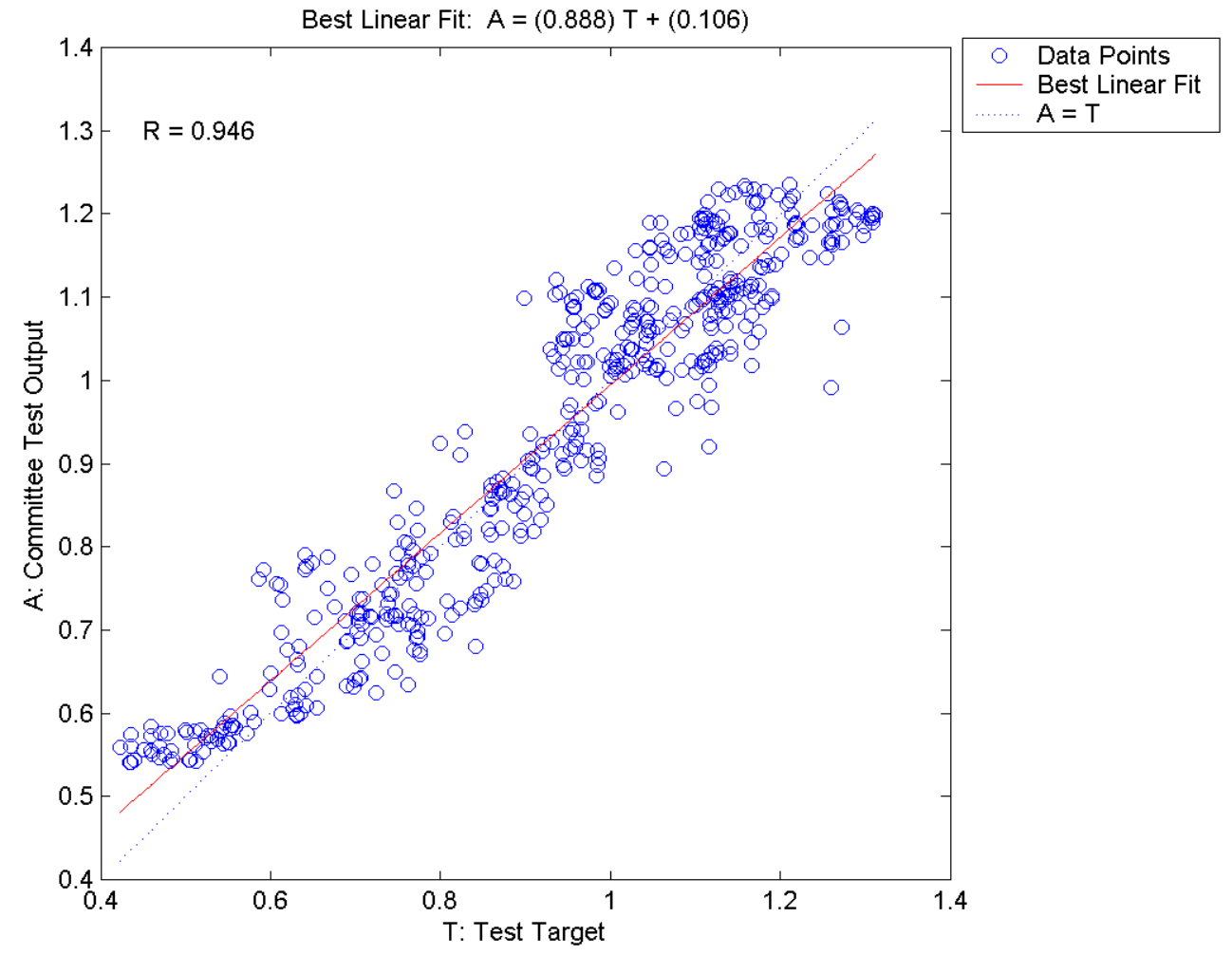

Figure 50 Network committee, test data performance correlation. 


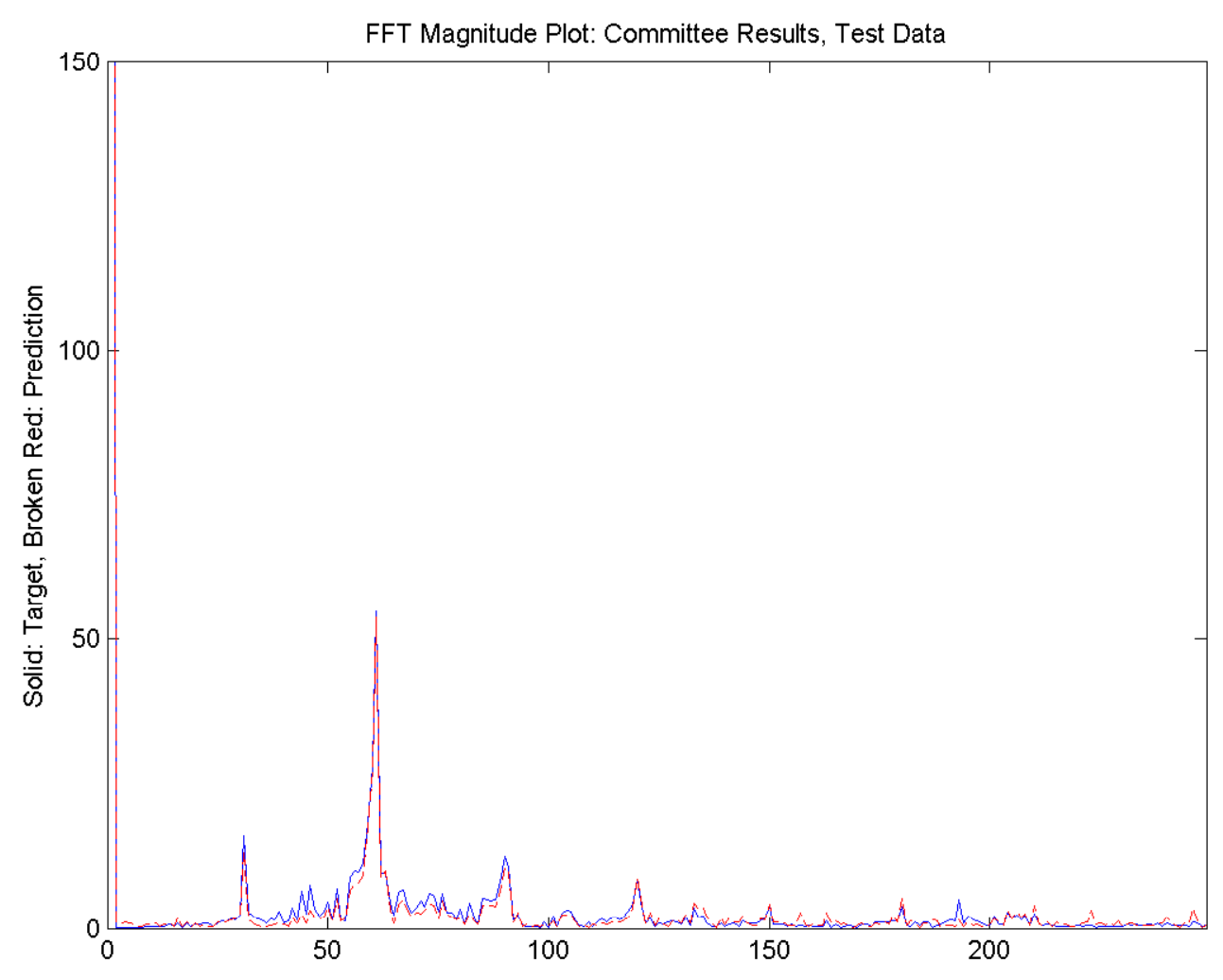

Figure 51 Network committee, test data Fourier transform magnitude plots.

\section{Comparison}

For comparison, three similar networks in terms of size and structure were used. A closely comparable standard temporal architecture to this two-node network is a 2node, two-layer focused TDNN. Three such networks were evaluated. Each is a two layer focused TDNN with one neuron in the hidden layer, one neuron in the output layer, and three different 11-branch input delay lines: $\left[\begin{array}{lllll}0 & 1 & 2 & \ldots & 10\end{array}\right],\left[\begin{array}{lllll}0 & 5 & 10 & \ldots & 5\end{array}\right]$ and $\left[\begin{array}{lllll}0 & 10 & 20 & \ldots\end{array}\right.$ 100]. The 11-tap input delay line was selected based on the size of a similar structure in the best-evolved network. The same training and test sets along with the same SCG training algorithm (same default) parameters were used. 
For the first focused TDNN, after several initializations these were the best results:

MSE train $=0.0230$

MSE test $=0.0240$

For the second focused TDNN, after several initializations these were the best results:

MSE train $=0.0482$

MSE test $=0.0489$

For the third focused TDNN these results were obtained:

MSE train $=0.0687$

MSE test $=0.0723$

Recall that the MSE for the GETnet evolved solution were 0.0052 for train and 0.0054 for test data. That is, the evolved network has found a structure that has a training and test MSE more than 4 to 13 times better than MSEs of similar focused time delay neural networks, as described above.

\section{Discussion}

The MG17 is a famous benchmark for time delay neural networks. Here it was observed how GETnet arrived at a compact solution that can perform the 6-step prediction task. The prediction closely tracks the target values in the time domain as can be seen from figures $32,33,37,38,42,43,47$, and 48 . Figures $30,35,40,45$, and 50 show a correlation coefficient of 0.948 for training and 0.946 for testing (time domain) 
data pairs. Furthermore, the MSEs for train and test data are 0.0052 and 0.0054, respectively. Some important observations can be made here:

1. There is almost no difference between the performance of the network on training and test data sets. This shows an excellent generalization based on minimization of the model variance through aggressive regularization and pruning. This is especially important since one can use all the valuable training data for final complete training without having to be overly concerned about setting aside validation sets, since extraneous free parameters are already taken out.

2. The best network and network committee results show no discernable differences. This is another indication of minimization of model variance, which the committee was supposed to cancel out through output averaging. However, committee will improve the results if the population of answers does not converge to an optimum.

It is also worth noting that the spectra of the prediction and target signals are almost identical in frequency domain (figures 36, 41, 46, and 51). This is important since the MG17 series is chaotic and pseudo periodic however the evolved neural network prediction is still closely following the target frequency contents using just two neurons.

Figure 31 shows the evolution of network size in terms of the number of branches (delayed weighed connections). As it can be seen, GETnet's strong tendency towards parsimony of the answers drives down the size of the evolved network sharply from the very beginning. However, after about 100 generations GETnet settles towards a solution that is slightly larger since the smaller networks were unable to improve the performance. Also note that through the course of evolution, the reduction of network size in terms of branches is 1.375 while the speedup in training time is about 3.6. This is what we desired by choosing a selection pressure that is related to the network complexity while emphasizing the actual execution time on the hosting hardware. 
As can be seen from the mean and median of the first and last best network in the evolution, the range of all mutation standard deviations has gone down many times while the MSE is improving. Especially, the weight perturbation standard deviation has reduced almost 30 times. This shows the convergence of the evolutionary search while it points towards the Baldwin effect, where the inherited garnered experience gradually replaces random mutations through guided evolution. This effect is also comparable to simulated annealing. It is interesting to note that this phenomenon was not dictated to the network, but it emerged from the evolutionary process. It is also interesting to take a closer look at figures 29 and 30, where the stepwise drops in the former figure and patchy grouping of individual fitness scores in the latter can be seen. The Baldwin effect was originally an attempt to describe punctuated equilibrium in natural evolution, and the jumps in figures 29 and 30 seem to suggest a similar phenomenon.

Finally, the comparisons show that the evolved network both on training and test sets does 4 to 13 times better than a regular similar TDNN. To make this comparison more tangible, the number of input branches for the base TDNN were chosen to be the same as the number that the evolutionary network had found. This might sound as hindsight in favor of the competing regular TDNN. Even so, one can see that the evolved network is still doing much better than the regular comparable networks by virtue of its evolutionary structural fine-tuning and hybrid training. 


\section{Mackey-Glass Chaotic Series 2}

For this simulation, the same Mackey-Glass time series as the previous experiment is used. However, the prediction task is six times deeper now. It will be shown here how GETnet finds a very compact architecture through evolution and hybrid training.

\section{Problem Description}

Mackey-Glass is a chaotic, non-periodic (pseudo-periodic), non-convergent univariate time series when $x(0)=1.2$ and $\tau=17$. The series' behavior is dependent on the values of the initial condition and the parameter $\tau$. The task is the 36 step predictions for the series.

\section{Data and Simulation Settings, 36-Step Prediction}

The first 1500 points of 6-sampled Mackey-Glass with $\tau=17$ data (MG17) was used in this simulation (see the generator $\mathrm{m}$ files and data source in the accompanying disk or the following FIRnet reference). The data itself was obtained from Eric Wan's benchmark collection of temporal data for FIRnet ${ }^{116}$. The sought task is a 36-step prediction. That is, given MG17(n), the network is to predict the value of MG17(n+36). Note that the data is resampled every 6 step so that each consecutive sample counts for a 6-point leap in MG17. That is, $\mathrm{x}(\mathrm{n}+6)$ refers to MG17(n+36). Thus GETnet is trying to find a model to estimate $f(x+36)$ from $\quad\{f(x), f(x-6), f(x-12), \ldots\}$. The first 500 samples (1 through 501 for input and 7 through 507 for target) are used for SCG partial training during the evolution loop, and the first 1000 samples (1 through 1001 for input and 7 through 1007 for target) are used for the corresponding validation score derived by Evaluate. The overlap of the seen first half and the unseen second half of the validation 
data is intentional. The contribution of the first half to the score accounts for the training quality of the network while the effect of the second half measures the generalization ability of the network under study. For GetCommittee, the initial validation data (1 through 1001 for input and 7 through 1007 for target) was used for complete post evolution SCG training and the rest of the data, i.e. 1008 through 1494 for input and 1014 through 1500 for the corresponding targets was used for test results.

\section{Results}

Here are the results of the best evolved network and committee of networks after postevolution training by GetCommittee:

Best_Net_MSE_Train $=0.0077$

Best_Net_MSE_Test $=0.0114$

Committee_MSE_Train $=0.0077$

Committee_MSE_Test $=0.0114$

Please refer to figures 53 through 77 for and the following discussion for more details.

General descriptors and strategy parameters:

1-Original ancestor of the best-evolved network:

size $($ total branches $)=33$

prune_threshold $=0.0227$

prune_threshold_SD $=0.0025$

node_mutation_SD $=0.1749$

connection_mutation_SD $=0.0243$

delay_mutation_SD $=0.6778$ 
Darwinian mutation' standard deviation (for Dar_inp_SD and Dar_lay_SD) $=0.0257$

Darwinian mutation' mean (for Dar_inp_SD and Dar_lay_SD) $=0.0465$

Connection weights' standard deviation $=0.4810$

Connection weights' mean $=0.1573$

Training/validation time (mean of multiple starts) $=53.7780 \mathrm{sec}$

2- Best evolved network, after 175 generations:

size $($ total branches $)=30$

prune_threshold $=0.0059$

prune_threshold_SD $=0.0001416$

node_mutation_SD $=0.1396$

connection_mutation_SD $=0.0702$

delay_mutation_SD $=0.00061462$

Darwinian mutation' standard deviation (for Dar_inp_SD and Dar_lay_SD) $=0.0023$

Darwinian mutation' mean (for Dar_inp_SD and Dar_lay_SD) $=0.0016$

Connection weights' standard deviation $=0.1835$

Connection weights' mean $=0.0435$

Training/validation time (mean of multiple starts) $=4.4010 \mathrm{sec}$ 


\section{Connection maps:}

1- Connection maps of the original ancestor of the best-evolved network

$$
\begin{gathered}
\text { input_connect }=\left[\begin{array}{l}
1 \\
1 \\
0 \\
1
\end{array}\right] \\
\text { layer_connect }=\left[\begin{array}{llll}
0 & 0 & 0 & 0 \\
0 & 0 & 1 & 1 \\
1 & 0 & 0 & 1 \\
0 & 1 & 0 & 0
\end{array}\right] \\
\text { output_connect }=\left[\begin{array}{l}
0 \\
1 \\
0 \\
0
\end{array}\right]
\end{gathered}
$$

2- Connection maps of the best-evolved network after 175 generations:

$$
\begin{aligned}
& \text { input_connect }=[1] \\
& \text { layer_connect }=[0] \\
& \text { output_connect }=[1]
\end{aligned}
$$

\section{Weights}

1- Connection weights, original ancestor of the best-evolved network: 


$$
\begin{aligned}
& \text { input_weights }=\left[\begin{array}{l}
\overrightarrow{i w}_{11} \\
\overrightarrow{i w}_{21} \\
{[]} \\
\overrightarrow{i w_{41}}
\end{array}\right] \\
& \text { layer_weights }=\left[\begin{array}{cccc}
{[]} & {[]} & {[]} & {[]} \\
{[]} & {[]} & \overrightarrow{l w}_{23} & \overrightarrow{l w}_{24} \\
\overrightarrow{l w_{31}} & {[]} & {[]} & \overrightarrow{l w}_{34} \\
{[]} & \overrightarrow{l w}_{42} & {[]} & {[]}
\end{array}\right]
\end{aligned}
$$

Starting with the following vector elements:

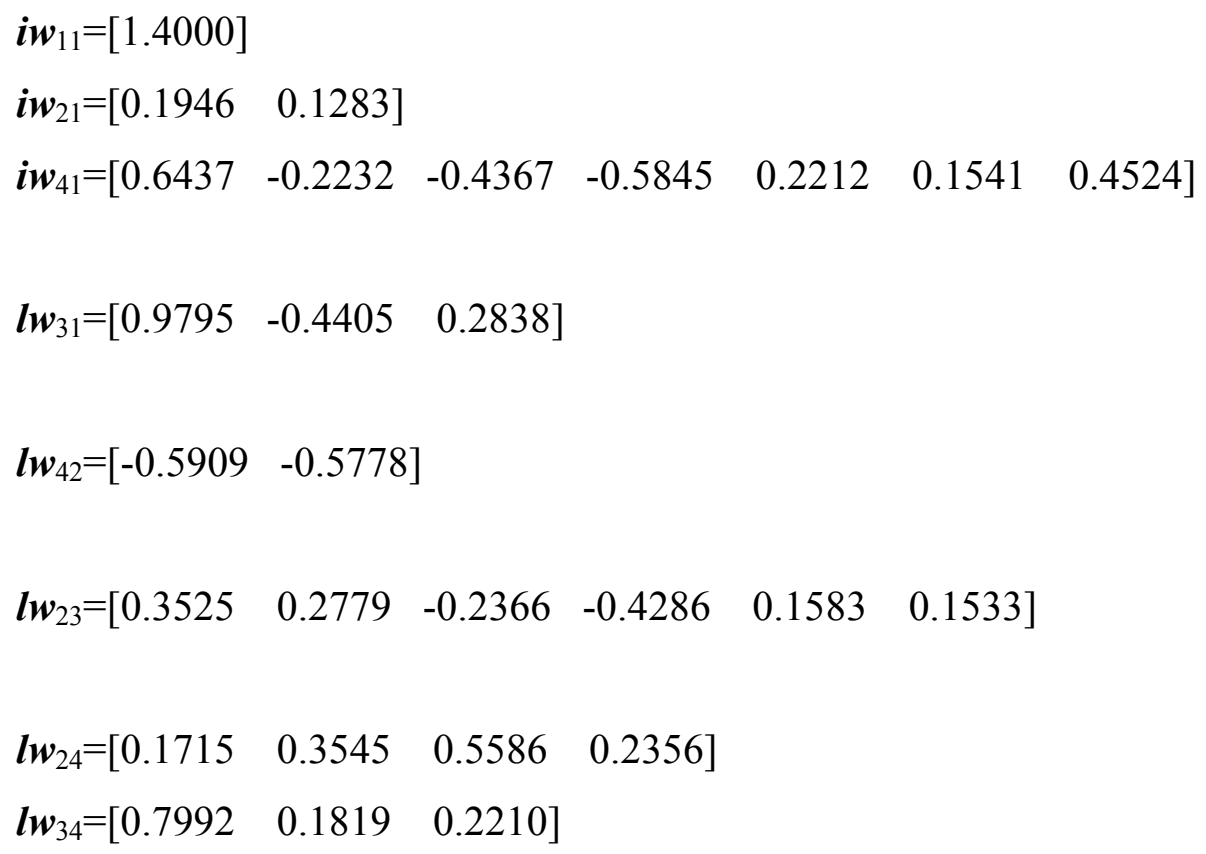

2- Connection weights, best evolved network after 175 generations:

$$
\begin{gathered}
\text { input_weights }=\left\lfloor\overrightarrow{i w}_{11}\right\rfloor \\
\text { layer_weights }=[]
\end{gathered}
$$


with the following vector element:

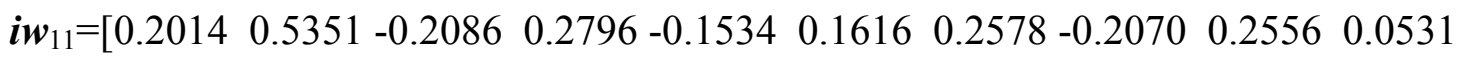

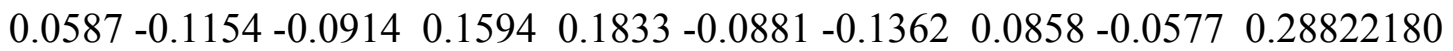

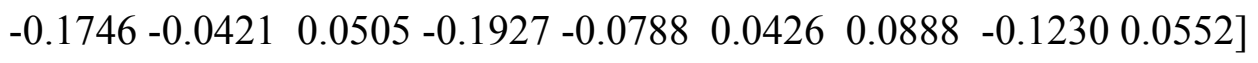

\section{Weight Evolution}

Evolutionary training of weight values is performed through individual Gaussian perturbations, which are determined by the standard deviation matrices given below.

1- Original ancestor of the best-evolved network evolution:

$$
\begin{aligned}
& \text { Dar_inp_SD }=\left[\begin{array}{c}
\overrightarrow{d i S D}_{11} \\
\overrightarrow{d i S D}_{21} \\
\frac{[]}{d i S D_{41}}
\end{array}\right]
\end{aligned}
$$

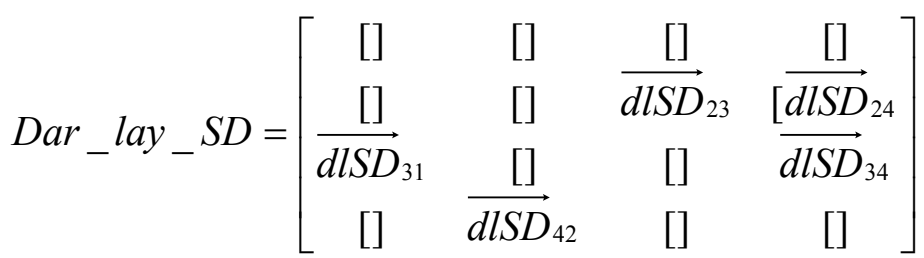

Starting with the following vector elements:

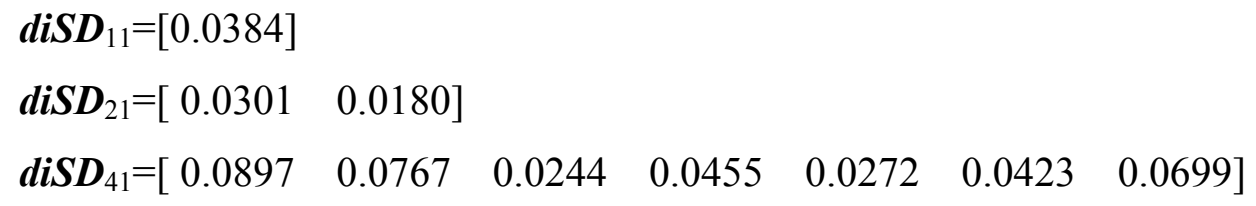




$$
\begin{aligned}
& d l S D_{31}=\left[\begin{array}{lll}
0.0256 & 0.0610 & 0.0447
\end{array}\right] \\
& d l S D_{42}=\left[\begin{array}{ll}
0.0340 & 0.0542
\end{array}\right] \\
& \boldsymbol{d l S D _ { 2 3 }}=\left[\begin{array}{llllll}
0.0044 & 0.0100 & 0.0957 & 0.0609 & 0.0247 & 0.0930
\end{array}\right] \\
& \boldsymbol{d l S D _ { 2 4 }}=\left[\begin{array}{llll}
0.0727 & 0.0489 & 0.0686 & 0.0363
\end{array}\right] \\
& d l S D_{34}=\left[\begin{array}{lll}
0.0149 & 0.0661 & 0.0237
\end{array}\right]
\end{aligned}
$$

2- Standard deviation matrices of weight perturbation, best-evolved network after 175 generations:

$$
\begin{gathered}
D a r_{-} i n p_{-} S D=\left|\overrightarrow{d i S D}_{11}\right| \\
D a r_{l} l a y_{-} S D=[]
\end{gathered}
$$

with the following vector elements:

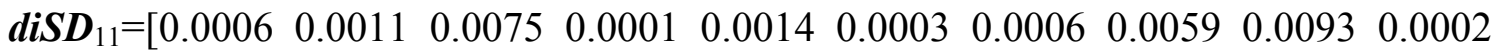

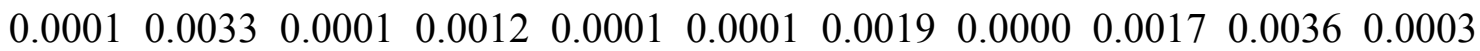
$\left.\begin{array}{lllllllll}0.0001 & 0.0037 & 0.0004 & 0.0000 & 0.0004 & 0.0018 & 0.0004 & 0.0004 & 0.0003\end{array}\right]$

Delays

1- Branch delays matrices of the original ancestor of the best-evolved network: 


$$
\begin{array}{r}
\text { input_delay }=\left[\begin{array}{c}
\overrightarrow{i d}_{11} \\
\overrightarrow{i d}_{21} \\
{[]} \\
\overrightarrow{i d}_{41}
\end{array}\right] \\
\text { layer_delay }=\left[\begin{array}{cccc}
{[]} & {[]} & {[]} & {[]} \\
{[]} & {[]} & \overrightarrow{l d}_{23} & \overrightarrow{l d}_{24} \\
\overrightarrow{l d}_{31} & {[]} & {[]} & \overrightarrow{l d}_{34} \\
{[]} & \overrightarrow{l d}_{42} & {[]} & {[]}
\end{array}\right]
\end{array}
$$

Starting with the following vector elements:

$$
\begin{aligned}
& i d_{11}=[6] \\
& \boldsymbol{i d}_{21}=\left[\begin{array}{ll}
1 & 4
\end{array}\right] \\
& \boldsymbol{i d _ { 4 1 }}=\left[\begin{array}{lllllll}
2 & 4 & 5 & 6 & 7 & 13 & 14
\end{array}\right] \\
& \boldsymbol{l d}_{31}=\left[\begin{array}{lll}
1 & 5 & 8
\end{array}\right] \\
& \boldsymbol{l d}_{42}=\left[\begin{array}{ll}
13 & 14
\end{array}\right] \\
& \boldsymbol{l d}_{23}=\left[\begin{array}{llllll}
3 & 6 & 7 & 11 & 13 & 14
\end{array}\right] \\
& \boldsymbol{l d}_{24}=\left[\begin{array}{llll}
1 & 10 & 14 & 15
\end{array}\right] \\
& \boldsymbol{l d} \boldsymbol{d}_{34}=\left[\begin{array}{lll}
2 & 6 & 11
\end{array}\right]
\end{aligned}
$$

2- Branch delays, best evolved network after 175 generations:

$$
\begin{aligned}
& \text { input_delay }=\left\lfloor\overrightarrow{\mathrm{id}}_{11} \mid\right. \\
& \text { layer_delay }=[]
\end{aligned}
$$


with the following vector elements:

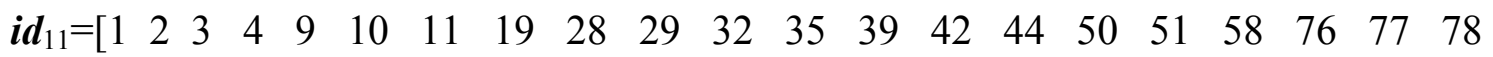
$\begin{array}{lllllllll}86 & 87 & 99 & 112 & 123 & 177 & 222 & 241 & 426]\end{array}$

The following figure shows the best evolved network.

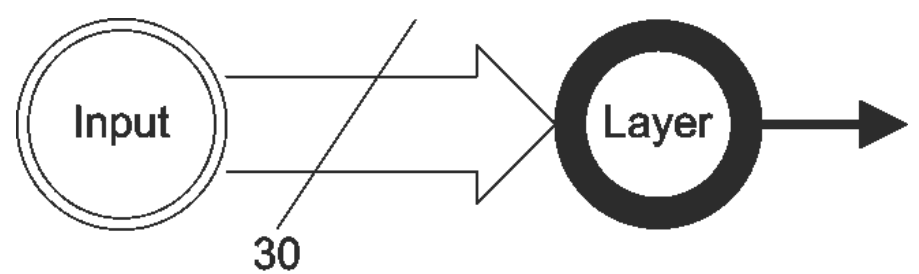

Figure 52 Best evolved network for MG17 thirty six-step prediction. There is a 30-line delayed synaptic connection between the input and the layer nodes. 


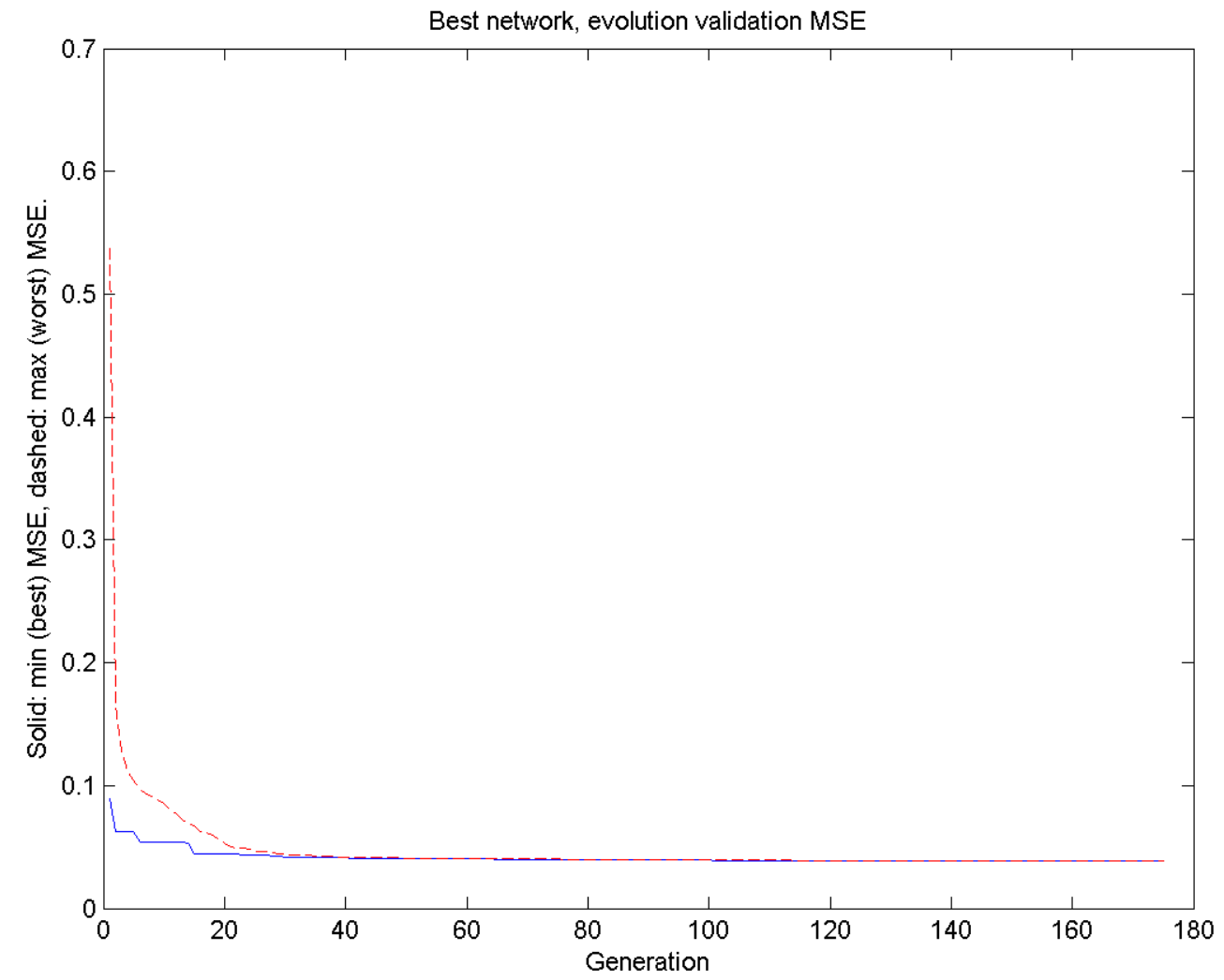

Figure 53 MSE of the evolving networks.

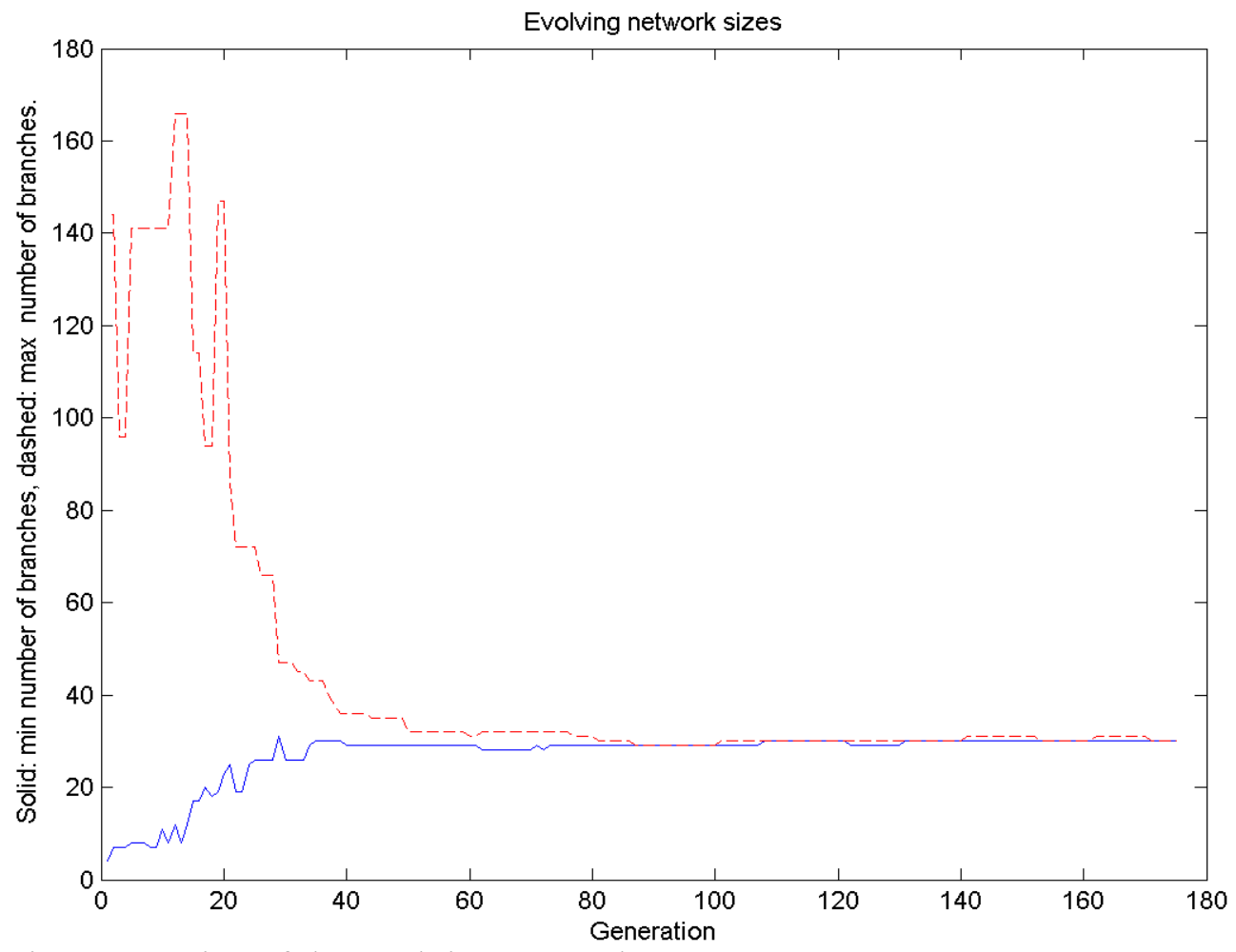

Figure 54 Size of the evolving networks. 


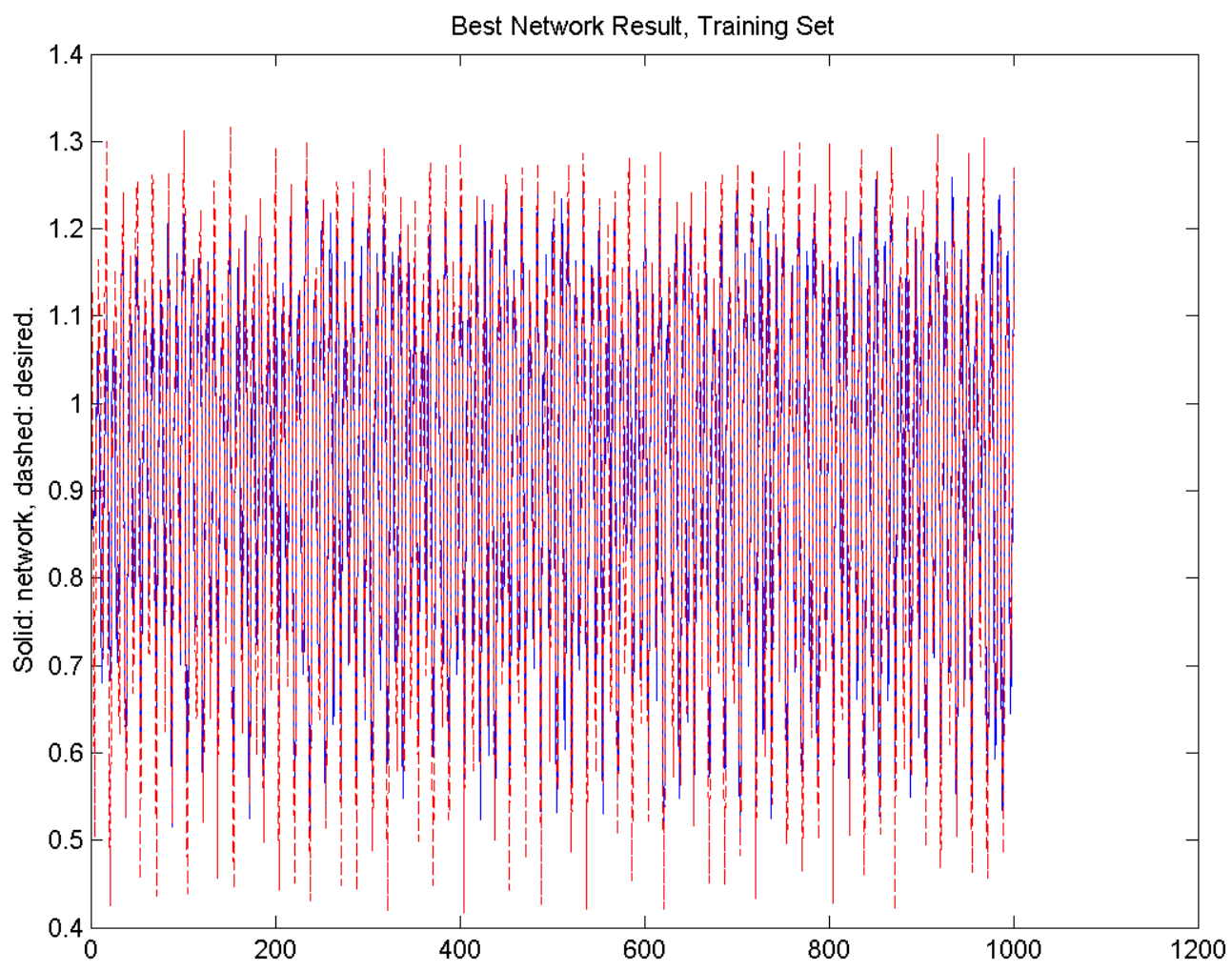

Figure 55 Training data, best evolved network.

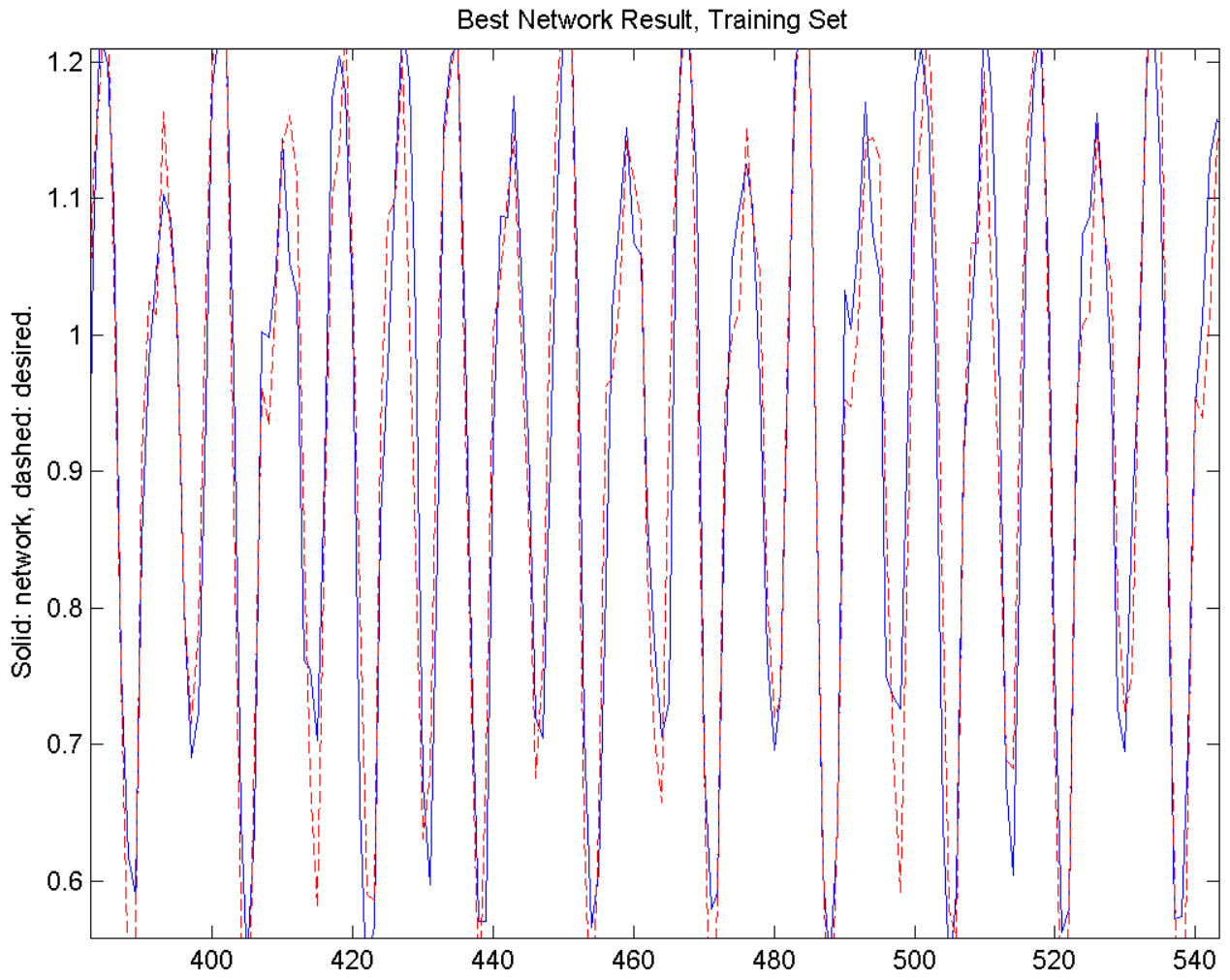

Figure 56 Training, magnified section for best evolved network. 


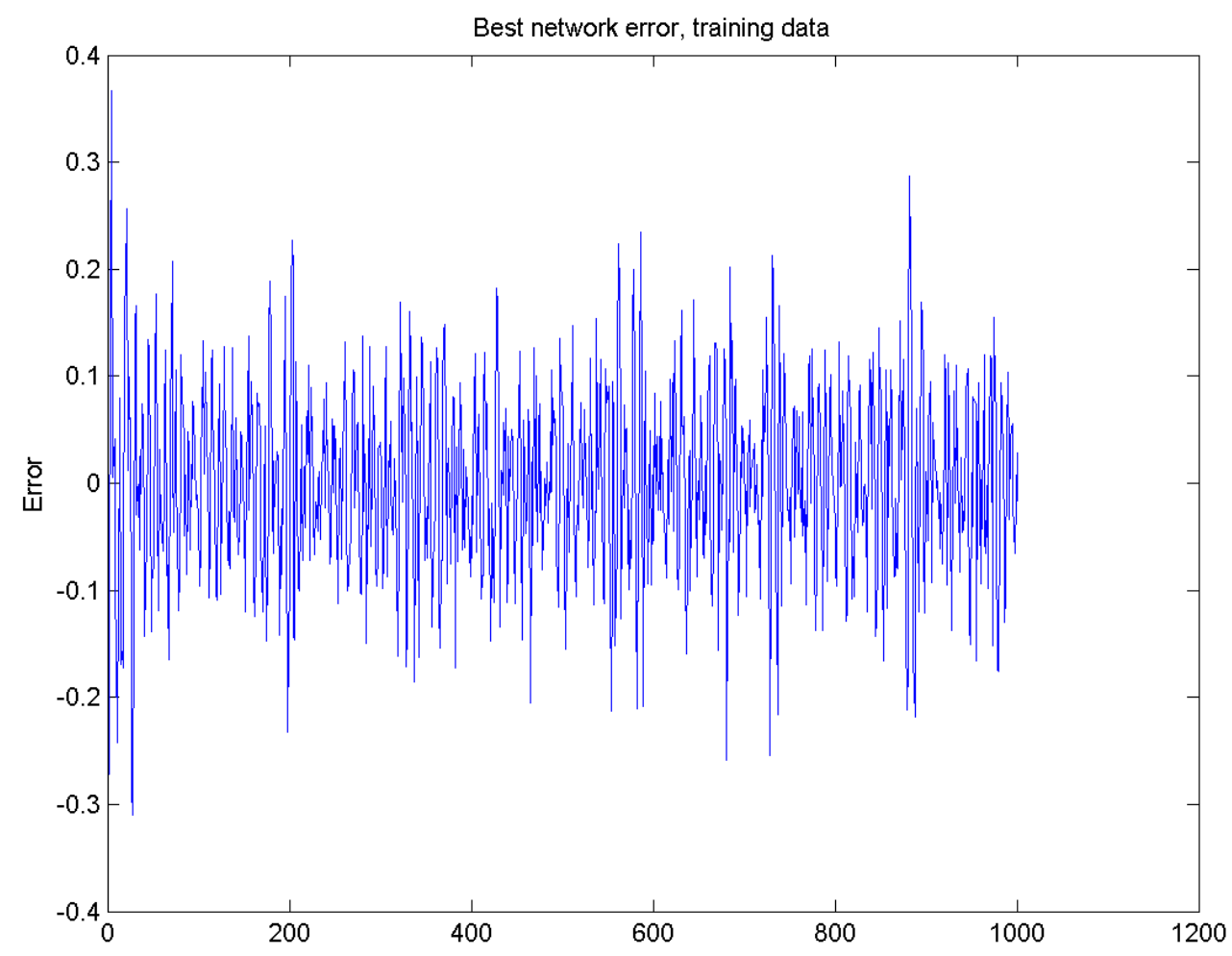

Figure 57 Best network, Training error.

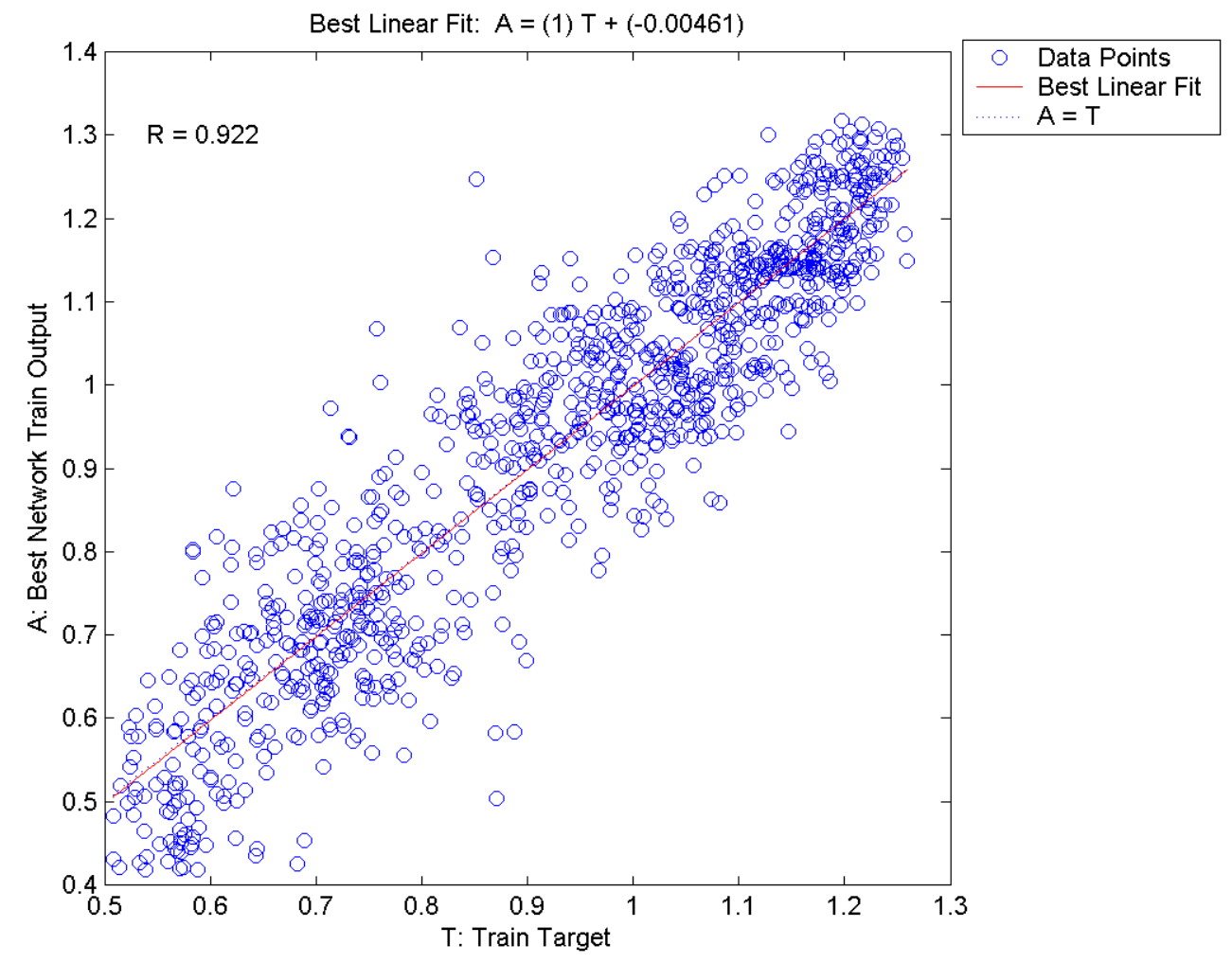

Figure 58 Best evolved network, training performance correlation. 


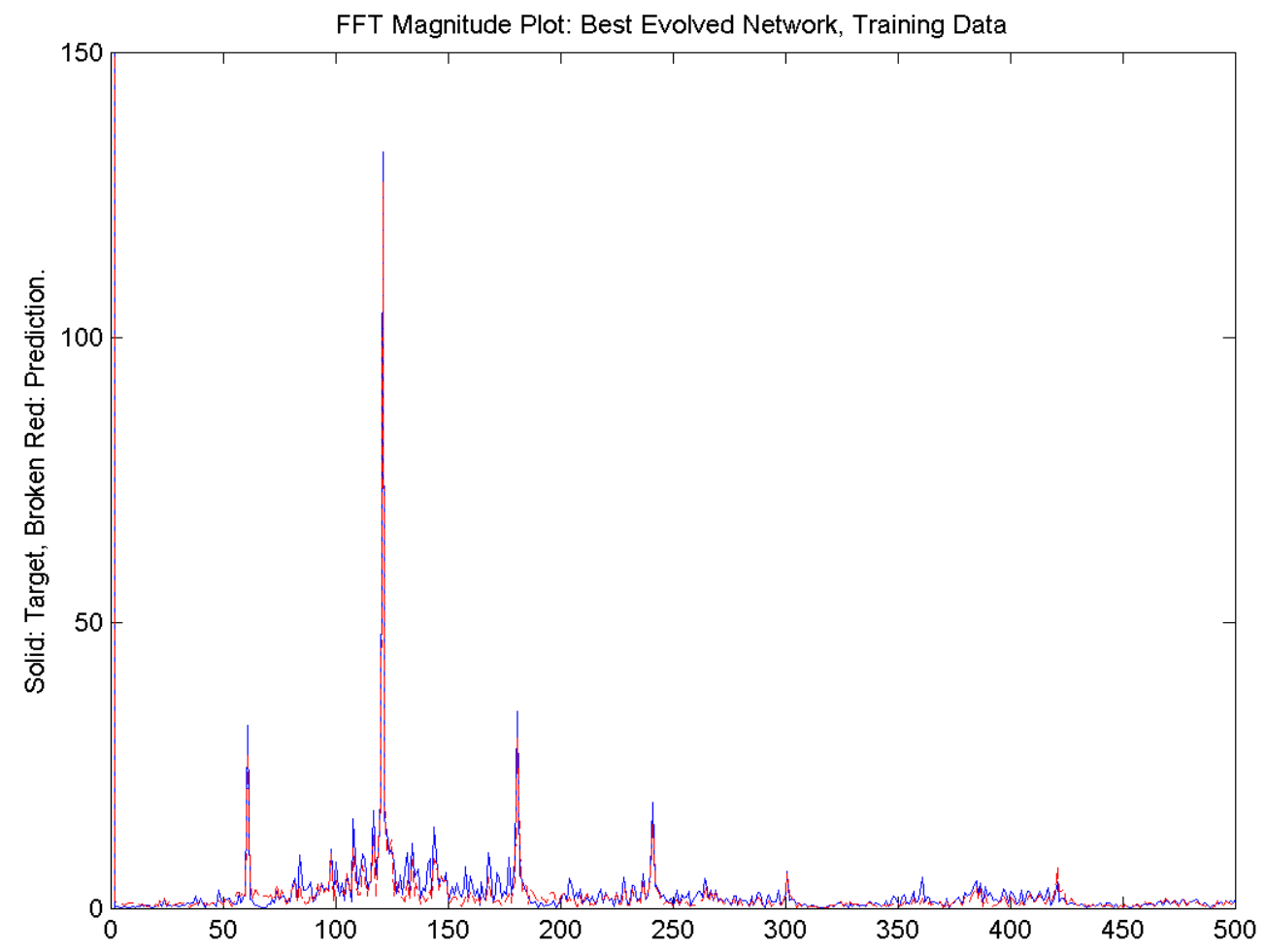

Figure 59 Best evolved network, training data Fourier transform magnitude plots.

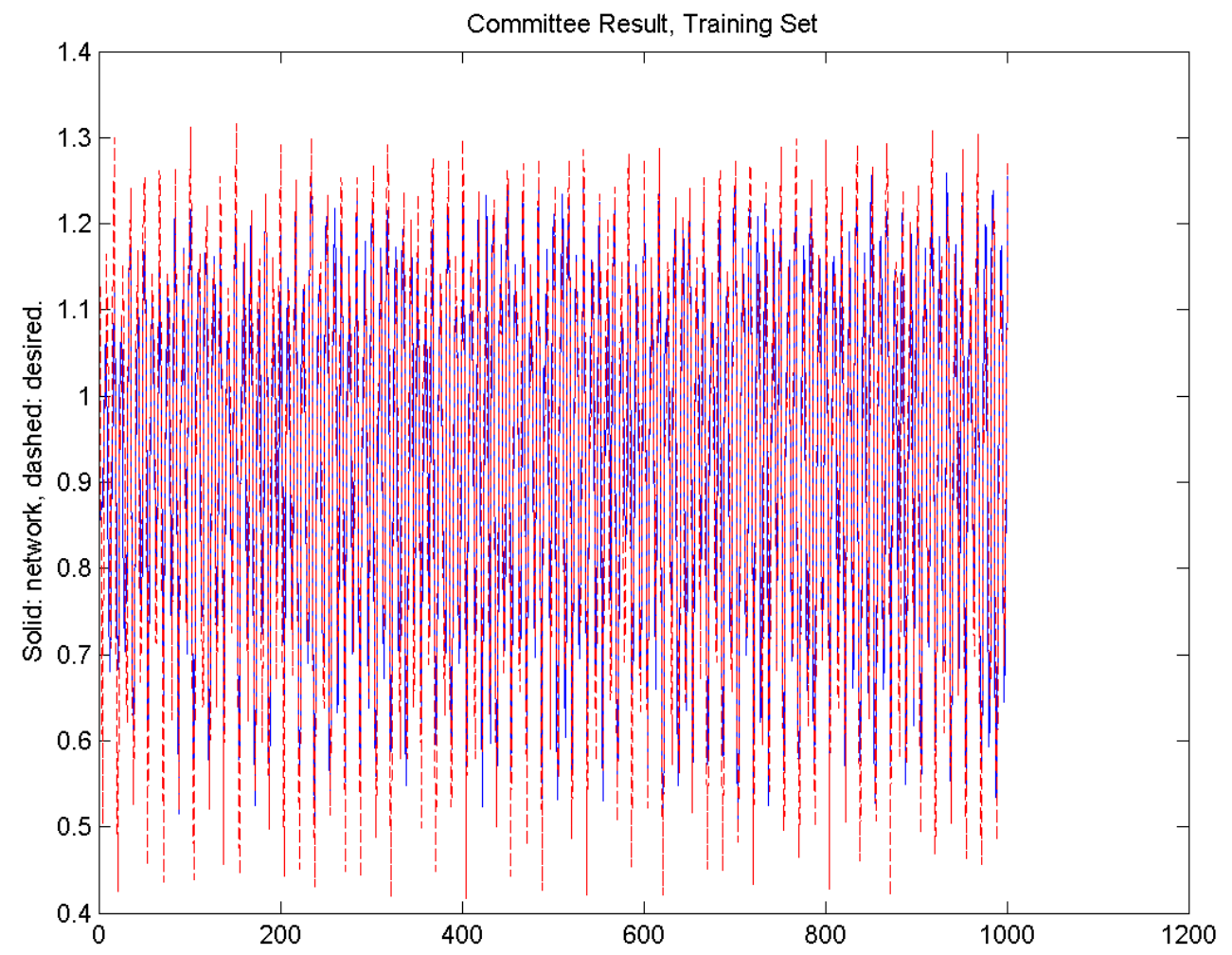

Figure 60 Training data, committee of last generation networks. 


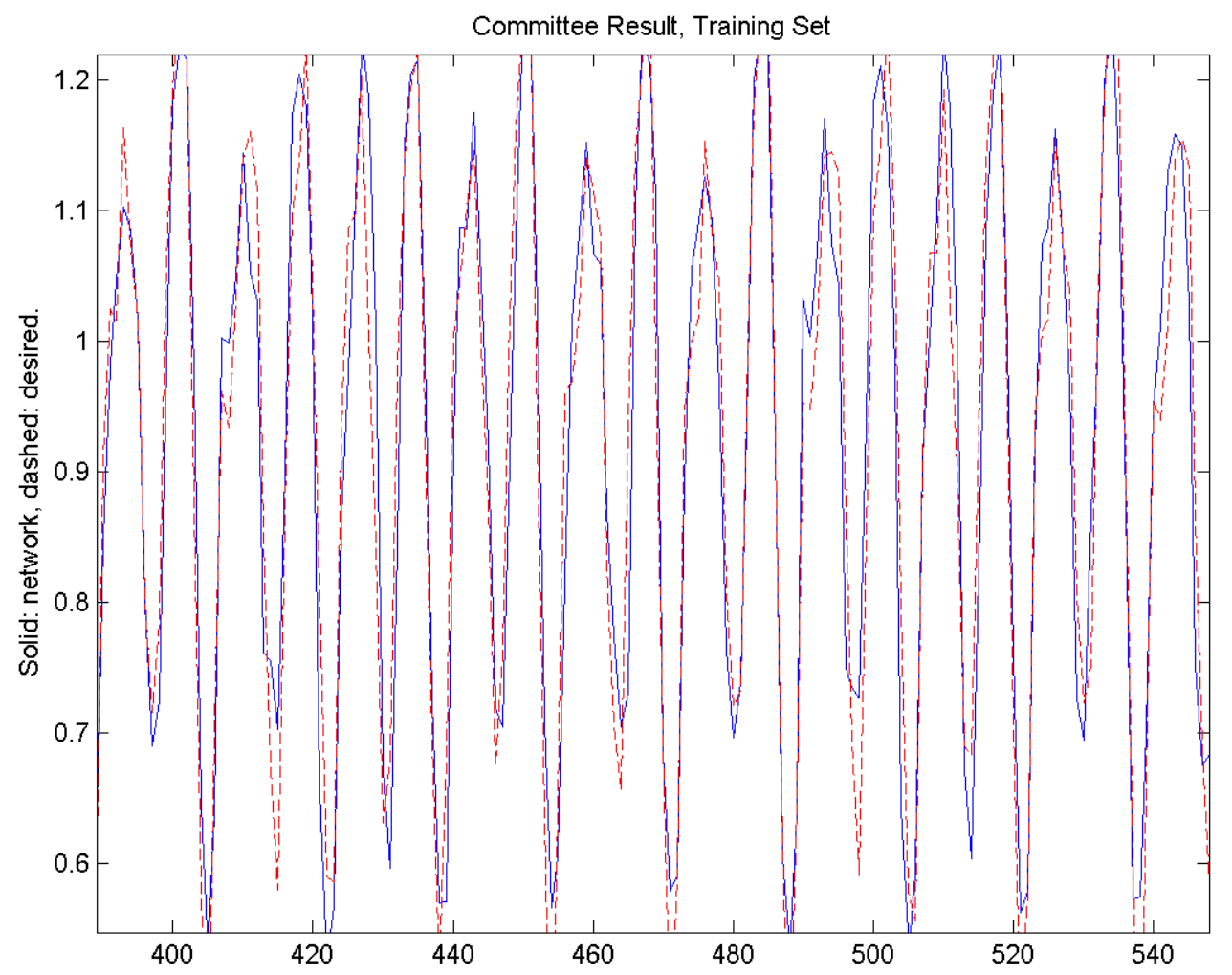

Figure 61 Training, magnified section for the network committee.

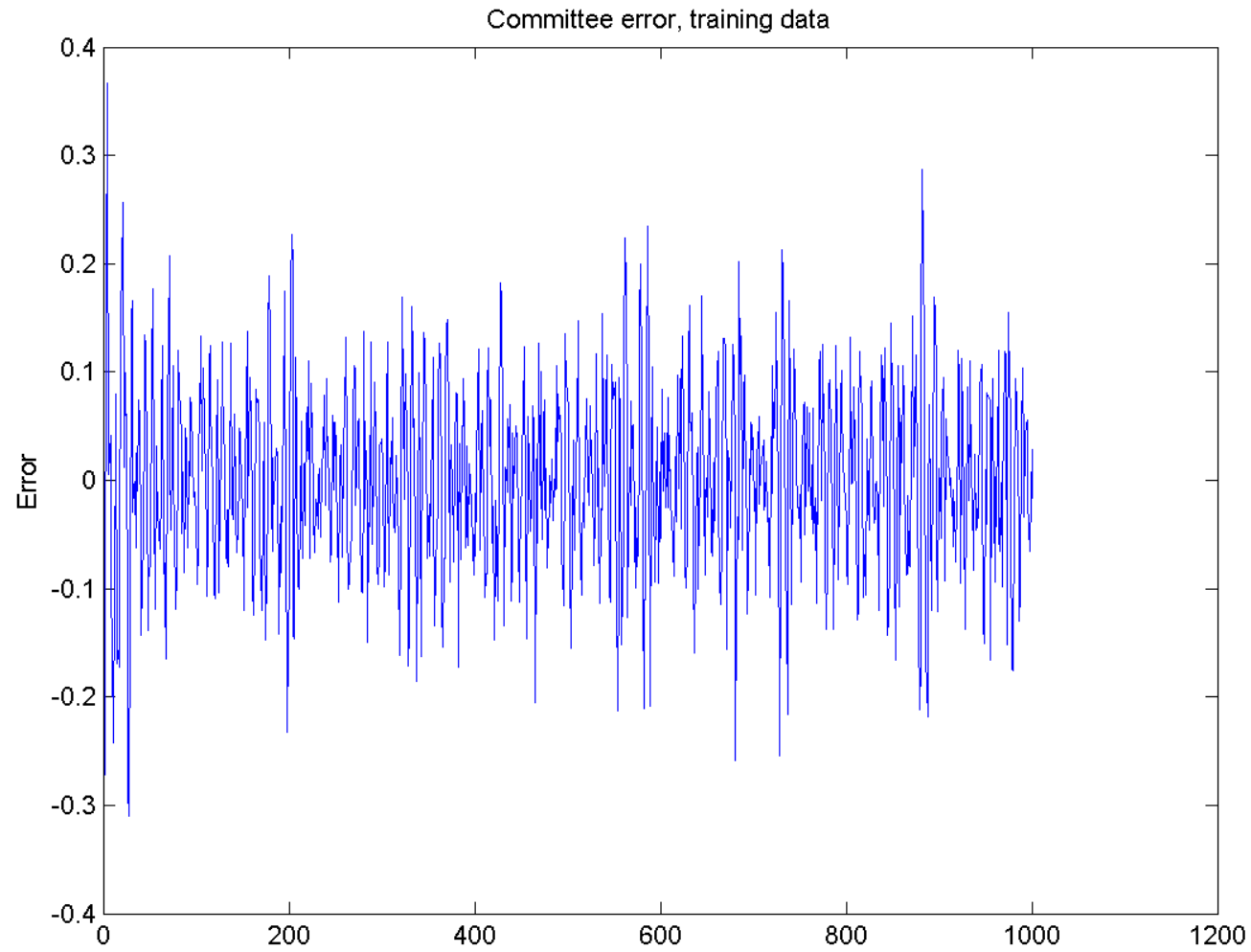

Figure 62 Network committee, training error. 


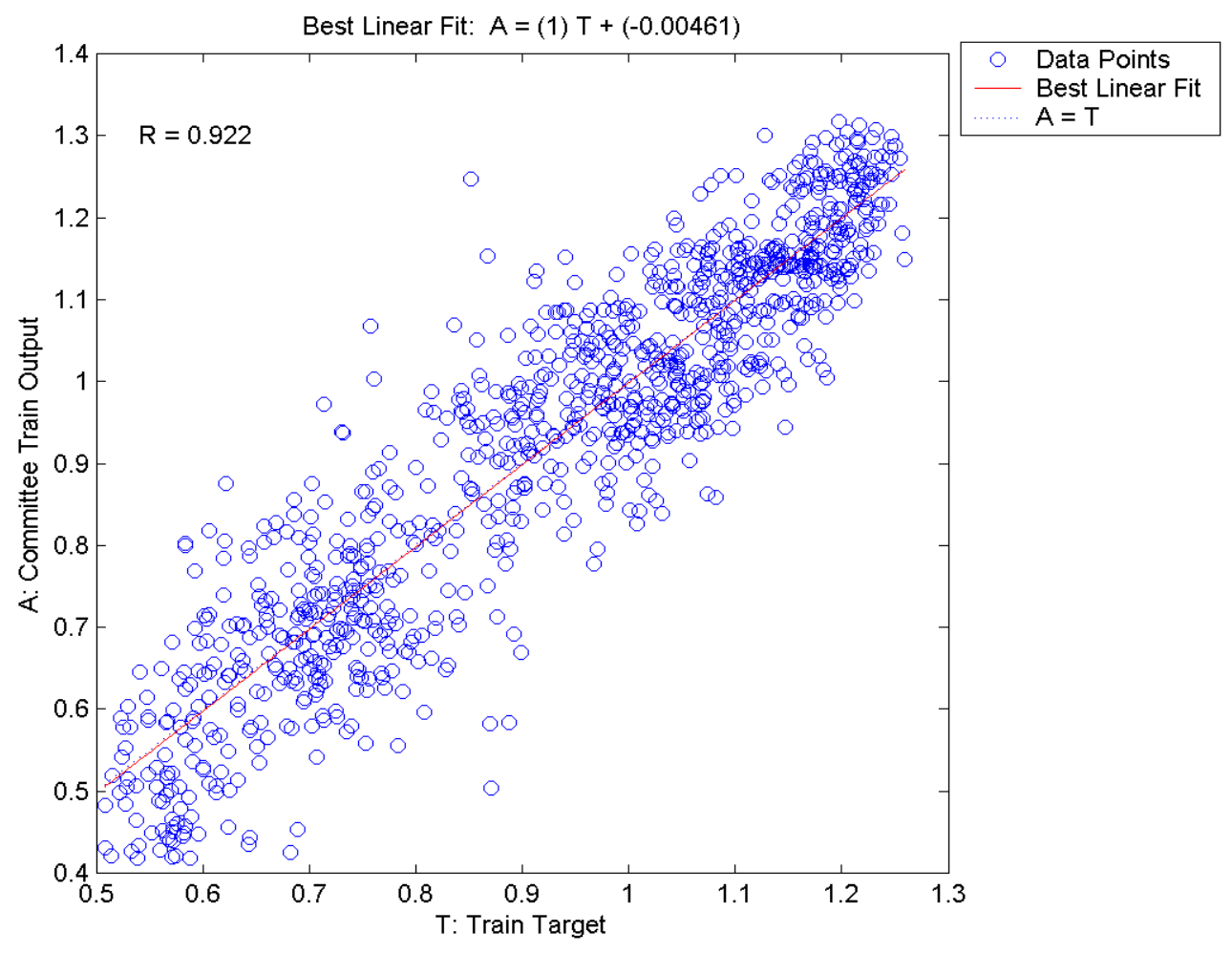

Figure 63 Network committee, training performance correlation.

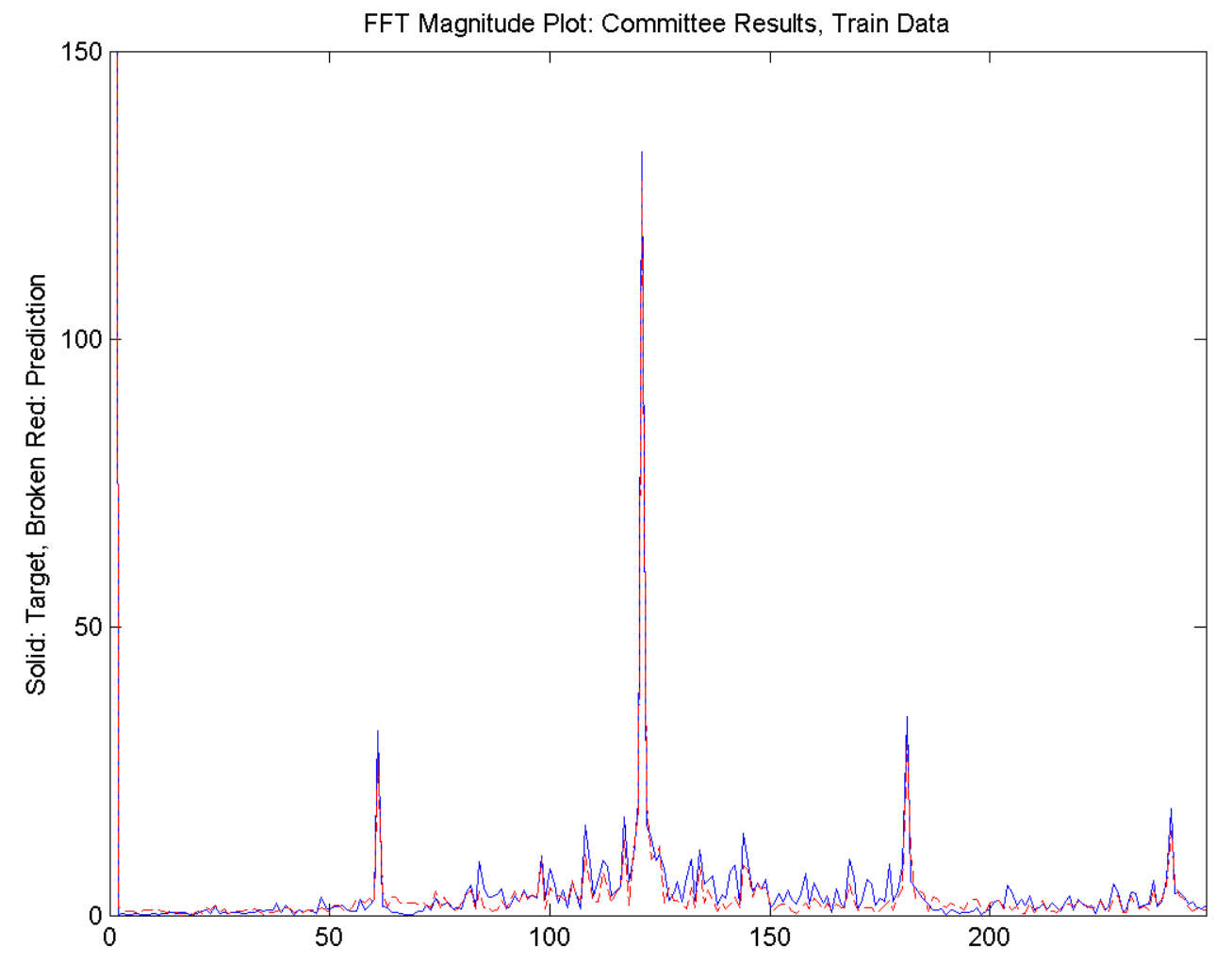

Figure 64 Network committee, training data Fourier transform magnitude plots. 


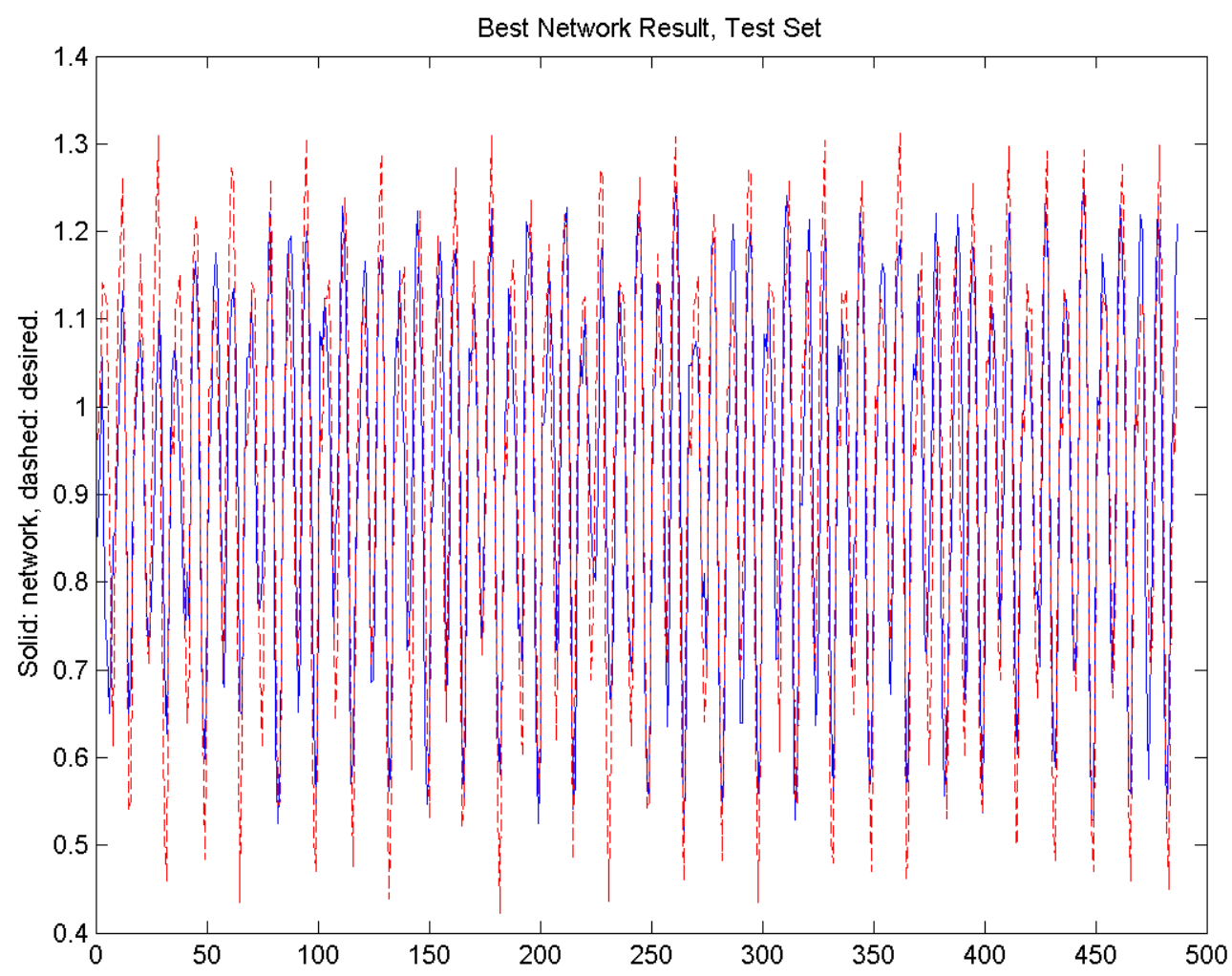

Figure 65 Test data, best evolved network.

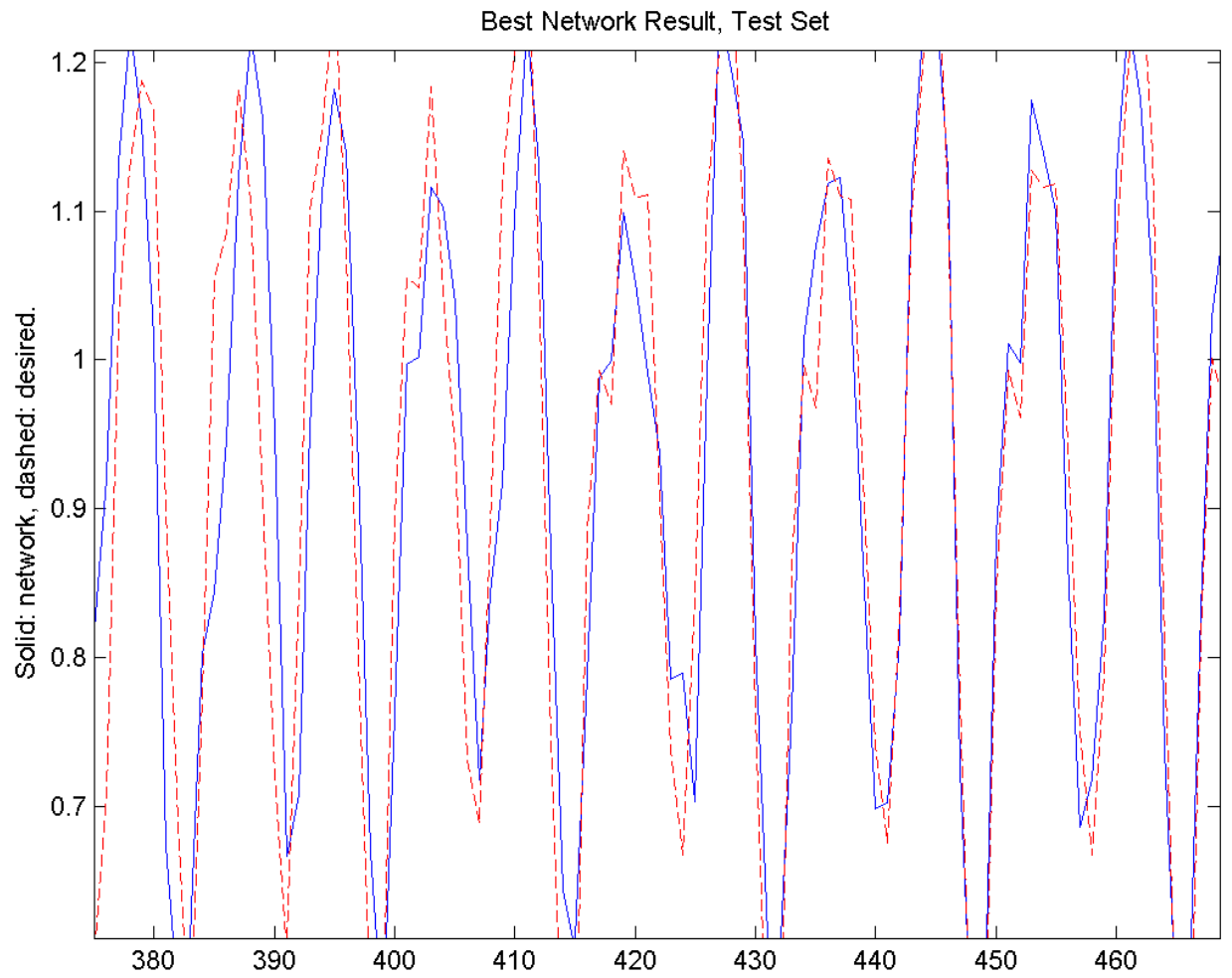

Figure 66 Test set performance, magnified section from the best evolved network. 


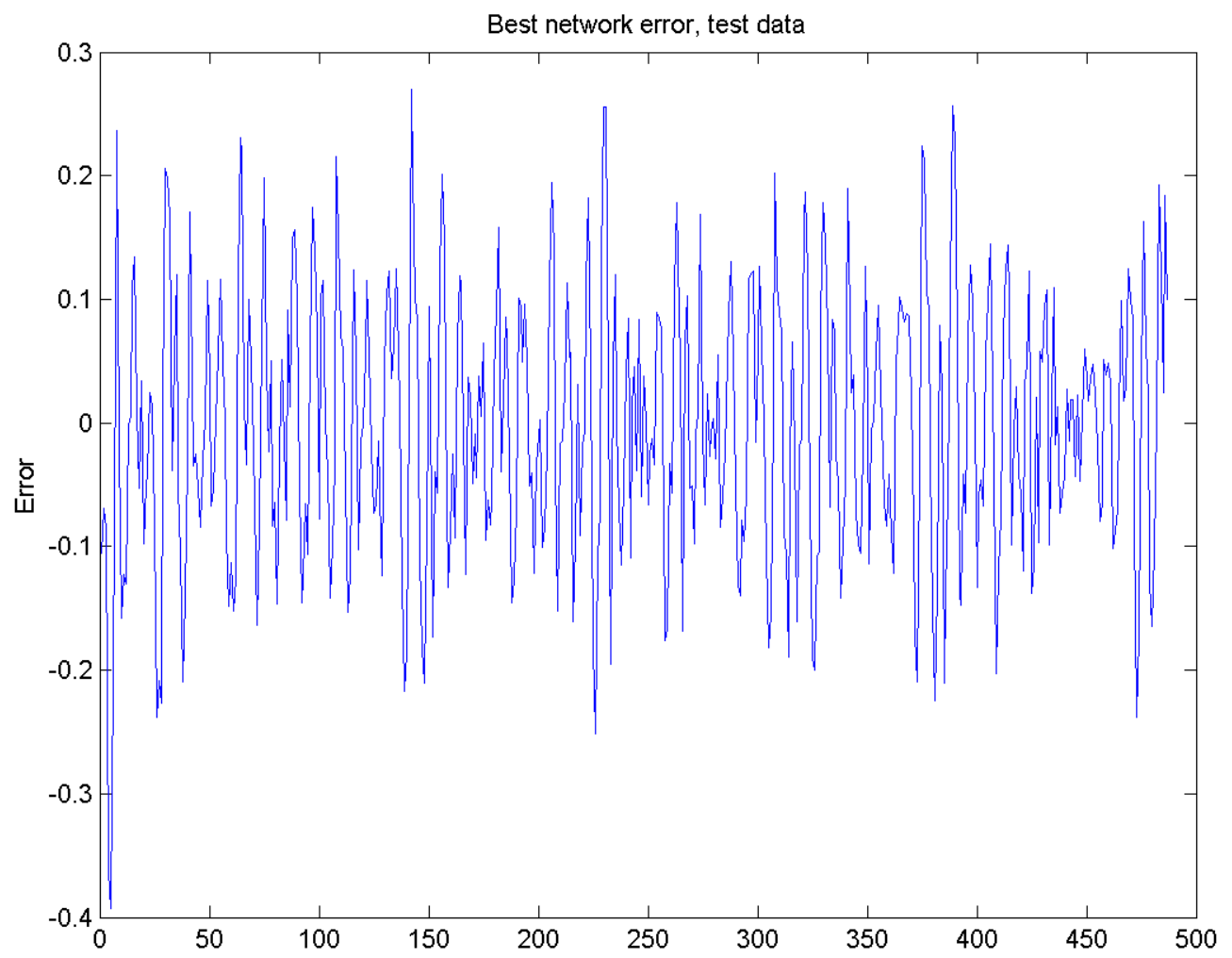

Figure 67 Best network, test error.

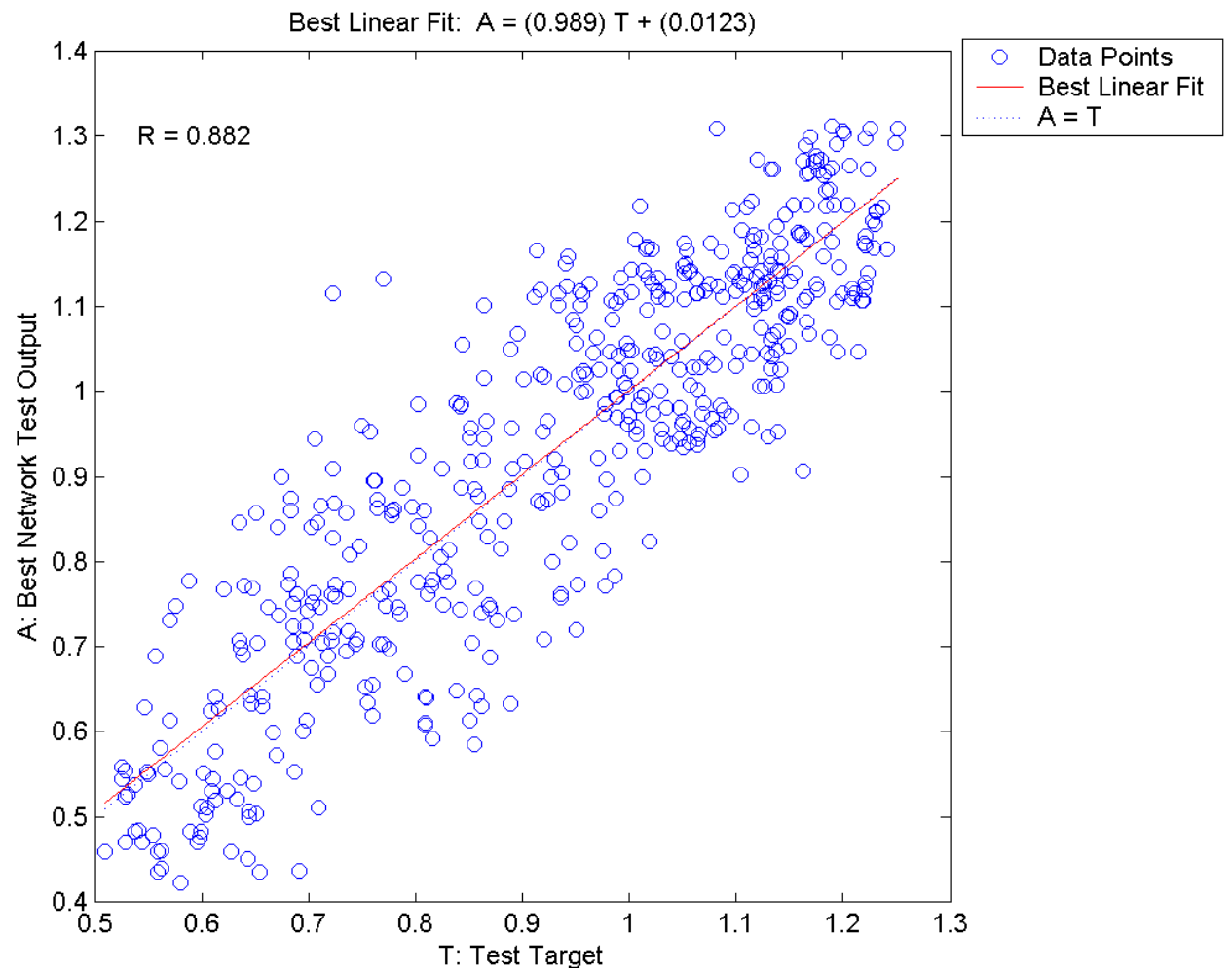

Figure 68 Best evolved network, test set performance correlation. 


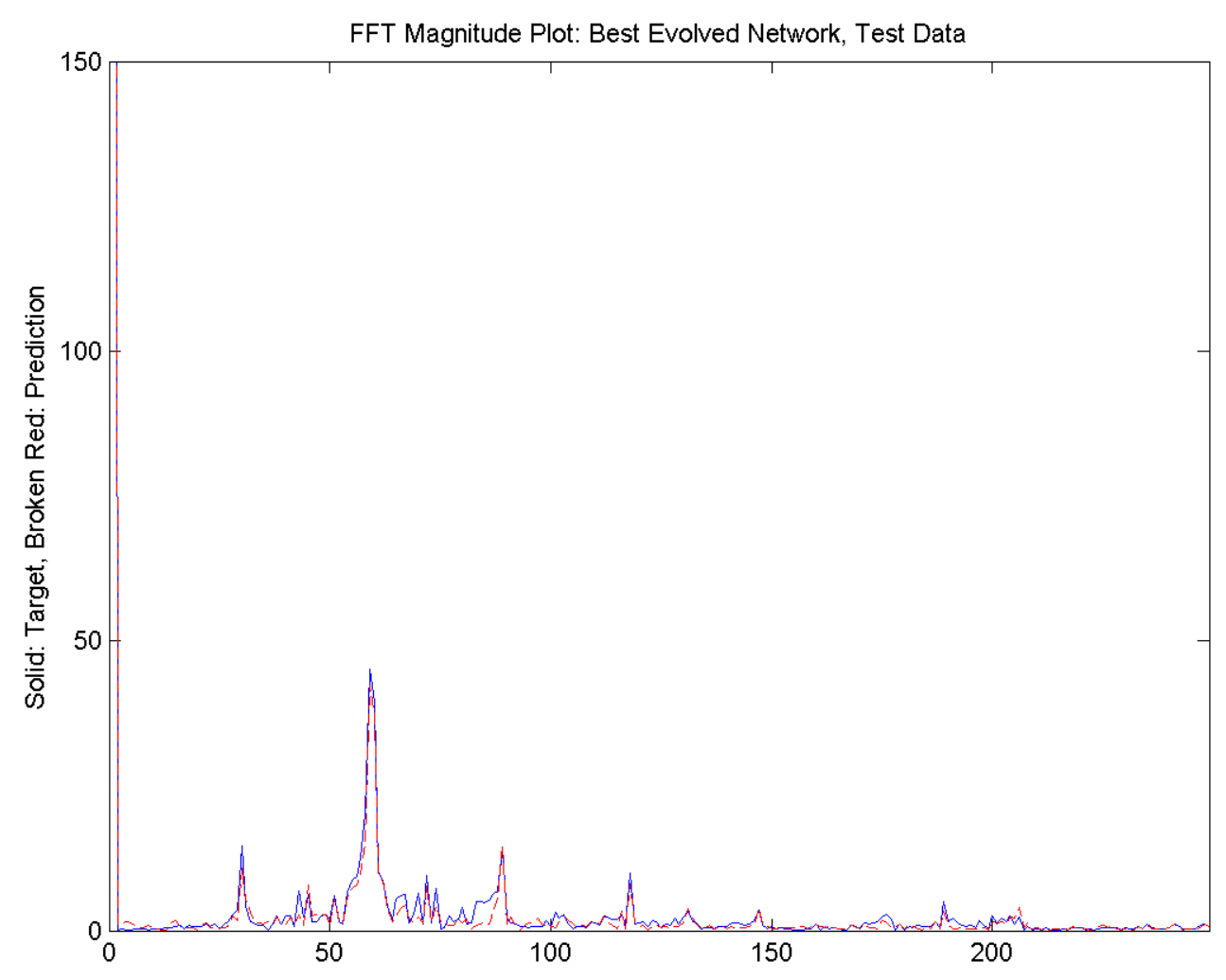

Figure 69 Best evolved network, test data Fourier transform magnitude plots.

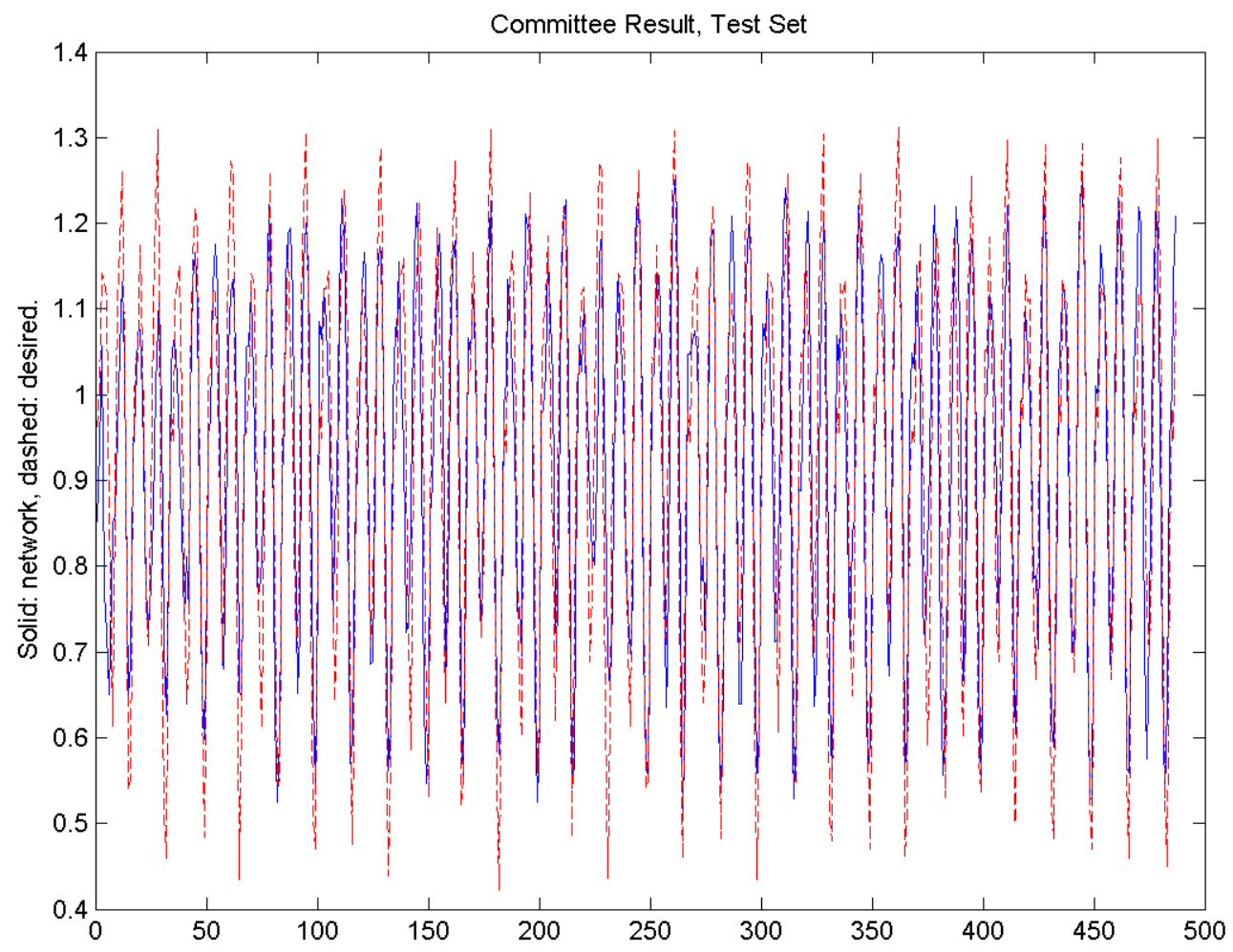

Figure 70 Test data, committee of last generation networks. 


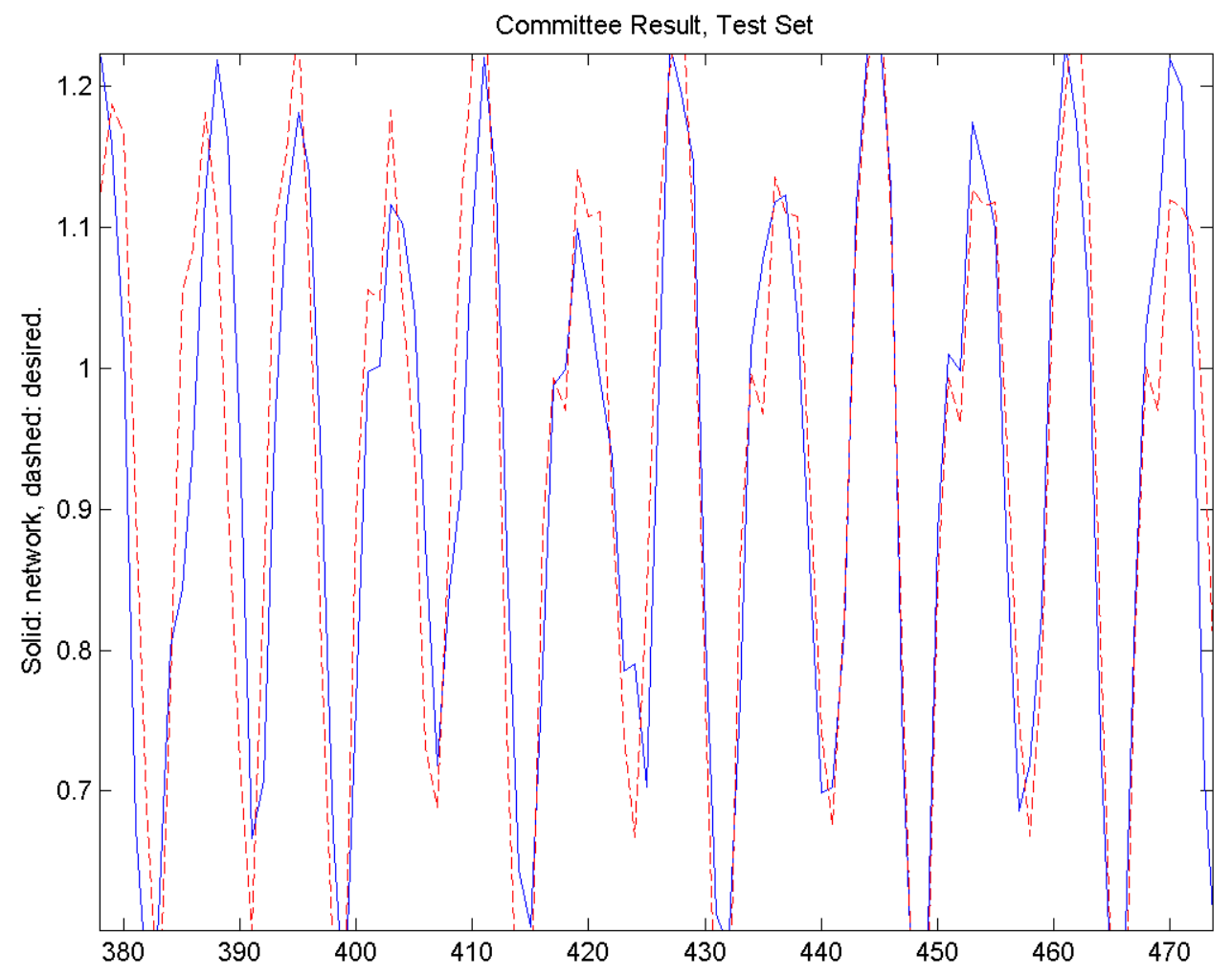

Figure 71 Test set performance, magnified section from the network committee.

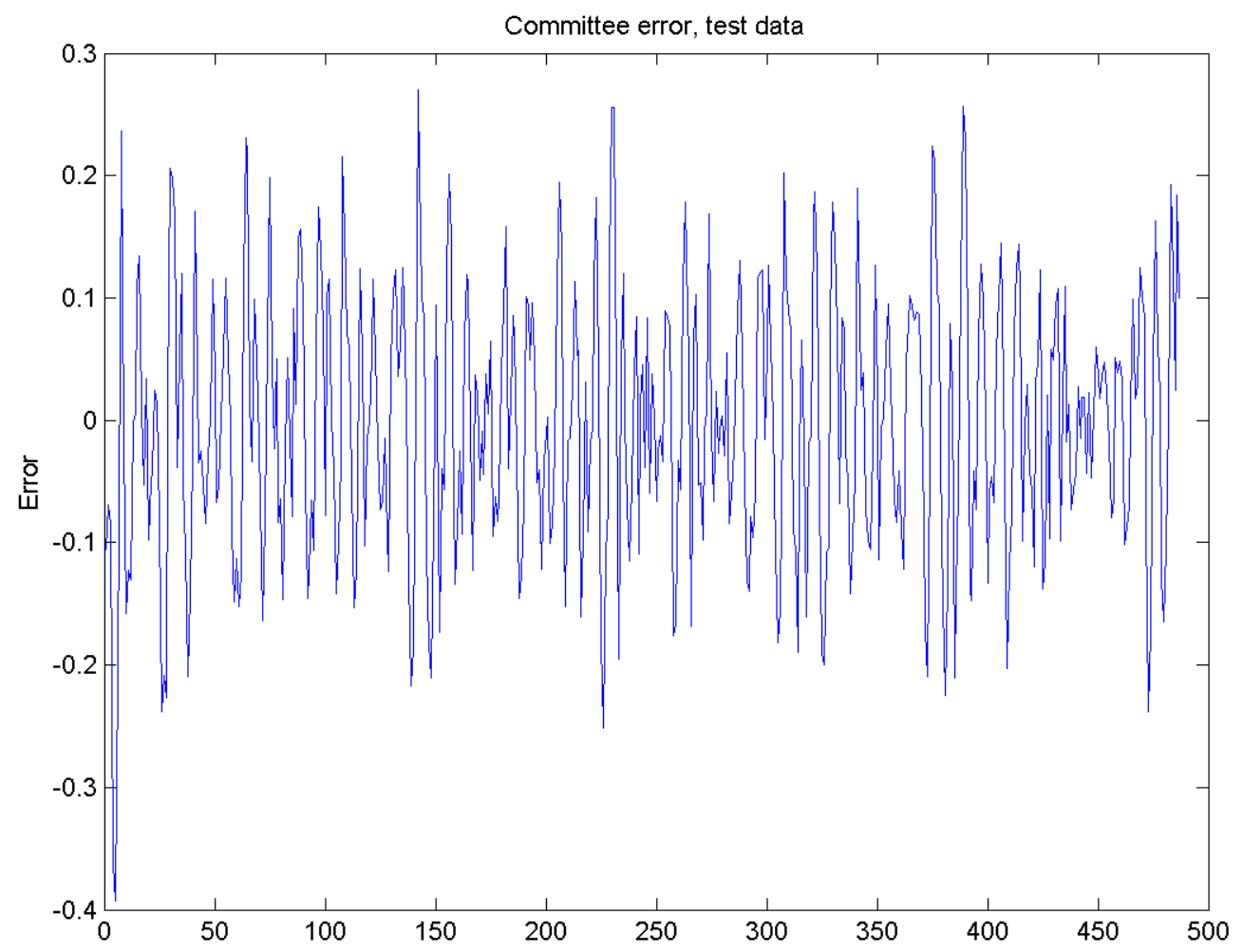

Figure 72 Network committee, test data error. 


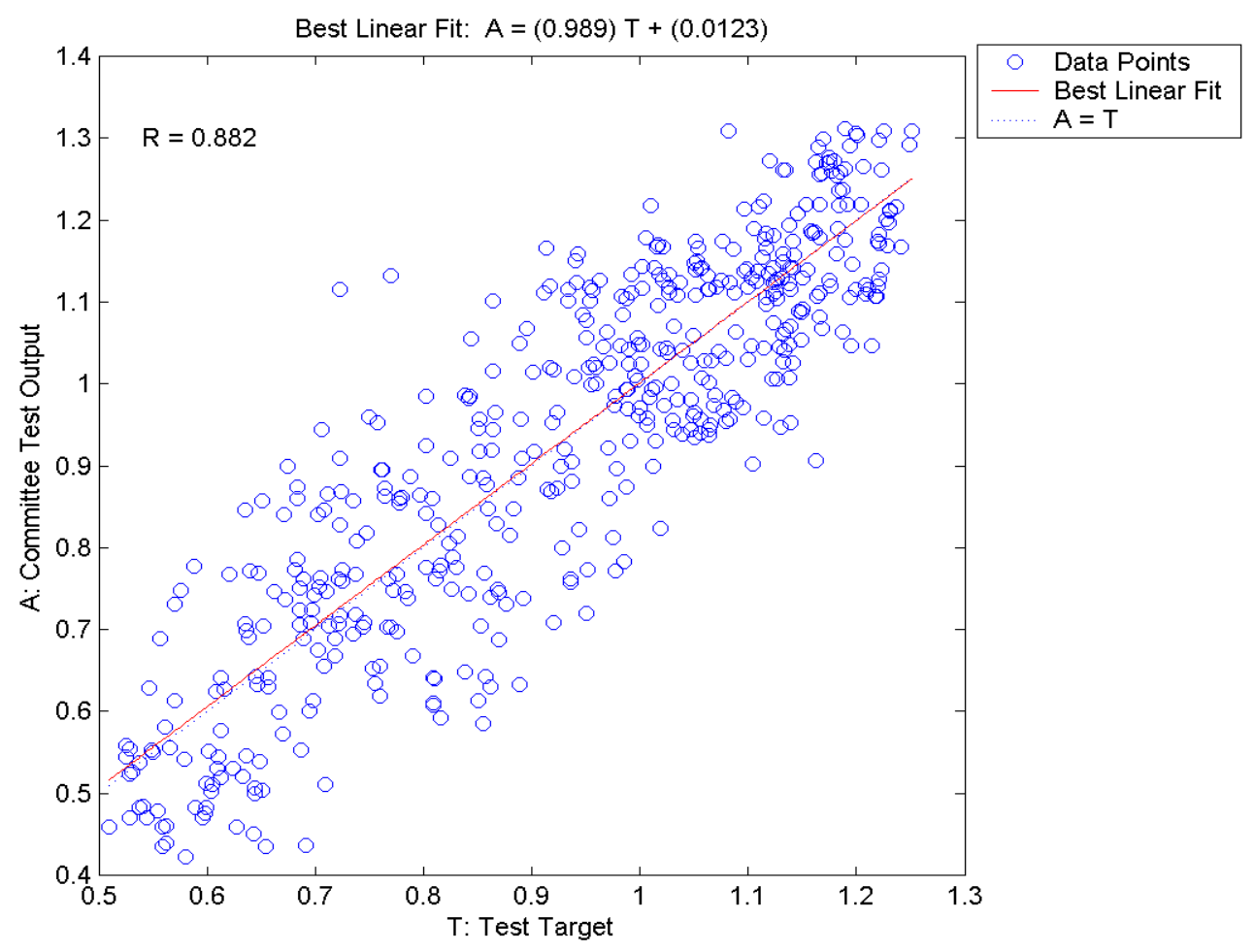

Figure 73 Network committee, test data performance correlation.

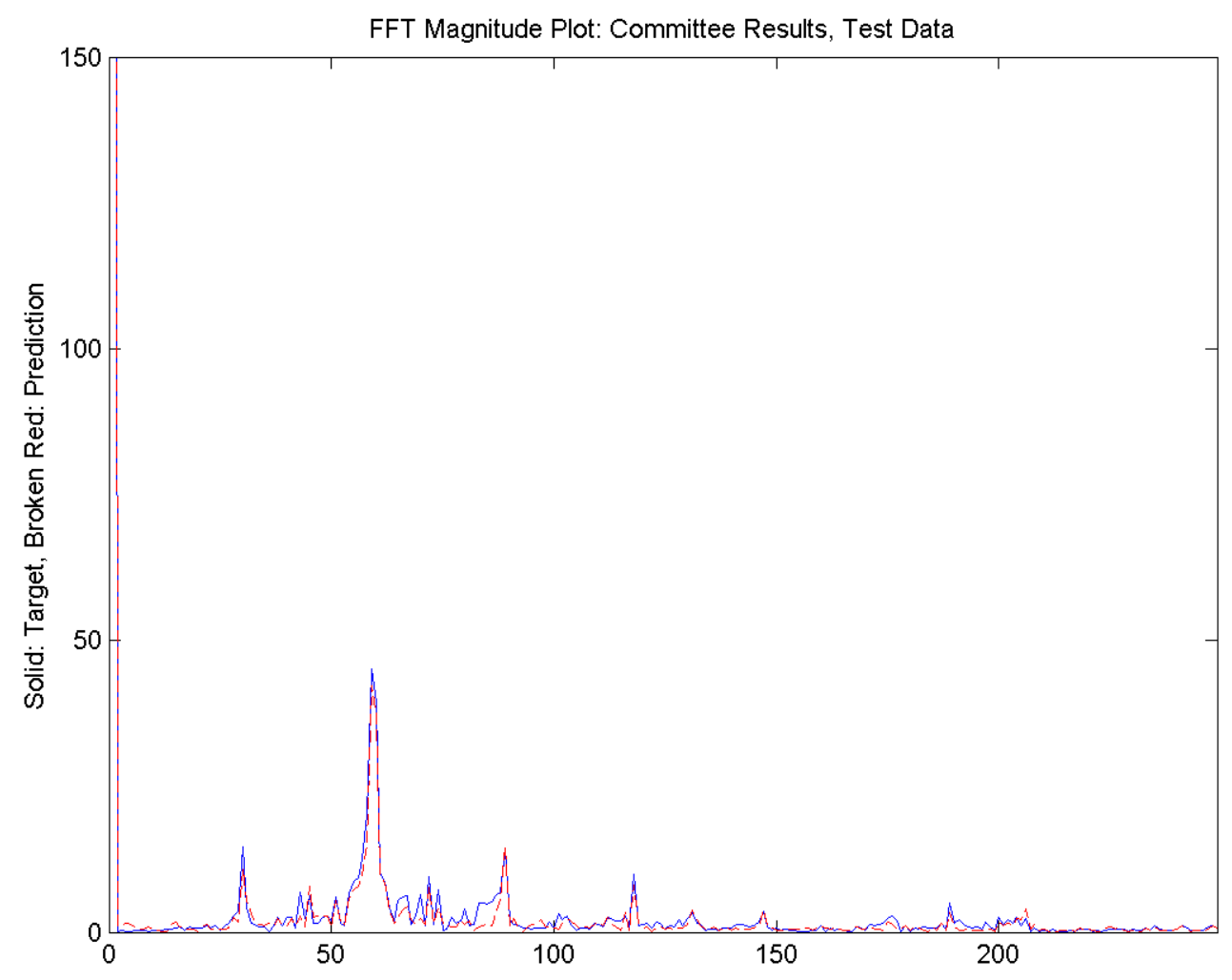

Figure 77 Network committee, test data Fourier transform magnitude plots. 


\section{Comparison}

For comparison, three similar networks in terms of size and structure were used. The single layer, single-neuron focused TDNNs used for comparison were given 30 input branches similar to that of the evolved network, with three different input delay line spacing. The same training and test sets along with same SCG training algorithm and default parameters were used.

For a single neuron with 30 input branches (focused TDNN with a delay window of 30 consecutive delays $\left.\left[\begin{array}{lllll}0 & 1 & 2 & \ldots & 29\end{array}\right]\right)$, these were the best results after several attempts with different initializations:

MSE train $=0.0378$

MSE test $=0.0476$

For another single neuron with 30 input branches (focused TDNN with a delay window of 30, 5-step apart delays $\left[\begin{array}{lllll}0 & 5 & 10 & \ldots & 145\end{array}\right]$ ) these were the best results after several attempts with different initializations:

MSE train $=0.0635$

MSE test $=0.0700$

For the last single neuron with 30 input branches (focused TDNN with a delay window of 30, 10-step apart delays $\left[\begin{array}{lllll}0 & 10 & 20 & \ldots 2\end{array}\right.$ 290]) these were the best results after several attempts with different initializations:

MSE train $=0.0716$

MSE test $=0.0903$ 
Recall that the MSE for the GETnet evolved solution were 0.00577 for train and 0.0114 for test data. That is, GETnet has found a structure that has a training MSE more than 4 to 9 times and test MSE more than 4 to 7 times better than that of similar focused time delay neural networks as described above.

\section{Discussion}

Following the previous benchmark test, this time the MG17 was applied for a 36step prediction task. After 175 generation, GETnet arrived at a very compact solution consisting of only 1 non-recurrent neuron with 30 parallel input branches from a 4neuron recurrent ancestor network. The prediction closely tracks the target values in the time domain as can be seen from figures 55, 56, 60, 61, 65, 66, 70, and 71. Figures 58, 63,68 , and 73 show a correlation coefficient of 0.922 for training and 0.882 for testing time domain data pairs. Furthermore, the MSEs for train and test data are 0.0077 and 0.0114 , respectively. The results are slightly worse than the previous task as expected, since this is a 6 fold deeper prediction.

As for the previous prediction task, there is only a small difference between the performance of the network on training and test data sets, which indicates the generalization capability acquired by minimizing model variance through aggressive regularization and pruning. This property of GETnet lets us use all the valuable training data for final complete training without having to be much concerned about setting aside validation sets, especially if the training data points are scarce. The similarity between best network and network committee results can also be explained in light of this reduced model variance.

Here too in the frequency domain the spectra of the prediction and target signals are almost identical (figures 59, 64, 69, and 77). This is important since the MG17 series 
is chaotic and pseudo periodic, and the evolved predicting neural network is able to almost duplicating the target frequency contents using just one neuron.

Figure 54 shows the evolution of the network size in terms of the number of branches (weights) per generation. As can be seen, GETnet's strong tendency towards parsimony of the answers reduces the size of the evolved network sharply from the very beginning and the population settles towards a solution after some transient fluctuations. Also note that through the course of evolution, the reduction of network size in terms of branches is 1.1 (or 4 times if number of nodes is considered) while the speedup in training time is about 12.2. This is what we desired by choosing a selection pressure that is related to the network complexity by utilizing the actual execution time on the hosting hardware, having in mind that simple weight counting is not a very good measure of system complexity.

As can be seen from the mean and median of the weight noise from the first and the last best network in the evolution, the range of all mutation standard deviations has gone down drastically reduced while the MSE has improved. Especially, the weight perturbation standard deviation mean has reduced about 29 times. This shows the convergence of the evolutionary search through generations, which is similar to simulated annealing. It also suggests the emergence of the Baldwin effect. Figure 53 also shows step-wise reductions in MSE after every several generations, resembling the punctuated equilibrium.

Finally, the comparisons show that the evolved network does 4 to 9 times better on training and 4 to 7 times better on test sets compared to a similar single layer, single node TDNN. To make this comparison more tangible, the number of input branches for the base TDNN were chosen to be the same as the number that the evolutionary network had found. This might sound as hindsight in favor of the competing regular TDNN. Even so, one can see that the evolved network is still doing much better than the regular 
comparable networks by virtue of its evolutionary structure fine-tuning and hybrid training. 


\section{Fingerprint Perspiration Sequence Detection}

In the following section, applicability of GETnet to a real world problem, fingerprint liveness detection, will be demonstrated. Note that it is the system capability rather than the benchmark that is of a concern here. The inputs are 2-D real-valued signals and the outputs are the corresponding 1-D classification signals.

\section{Brief Introduction}

There has been a growing interest in biometrics for verification or authentication of individuals under different scenarios. Not only for being historically one of the more popular biometrics, but also because of the introduction of cheap, small, and fast CMOS scanners, fingerprints have been receiving more attention. However, one of the associated security concerns is the possibility of intrusion by presenting a nonliving finger, be it a duplicate or a severed finger to an automated electronic fingerprint scanner in order to gain access to a protected entity. It has been shown that this threat is real and one can spoof fingerprint scanners even with play-doh ${ }^{117}$ and gummy fingers ${ }^{118,119}$.

In order to circumvent this problem, one can read signals from the finger that can verify its liveness and thus eliminate the threat of synthesized and cadaver finger attacks. However, reading the more obvious signs of life such as those obtained for electrocardiograms and pulse oximetry requires extra hardware. Earlier research of the author showed that the process of perspiration on live fingertip skin can be seen from the consecutive captures of electronic scanners within the first few seconds of each scan. The ongoing perspiration presents a specific time progression that cannot be seen in cadaver and synthetic fingerprint scans. This led to the development of an algorithm by the author that quantifies and subsequently detects liveness of fingerprints based on the aforementioned phenomena ${ }^{120}$. The algorithm uses two captures of a fingerprint in 5 
seconds, and concatenates the gray levels of the fingerprint ridges to obtain a ridge-signal that reflects moisture levels for each fingerprint capture (figure 78). Features from the ridge-signal pair (initial and after 5 seconds) are derived afterwards and fed to a classifier for final liveness decision.

It has been shown that other fingerprint capturing technologies such as optical and electro-optical scanners can record this process. It has also been shown that the perspiration based detection algorithm, originally developed for capacitive-DC CMOS scanners, is applicable to these other scanners with a varying degree of success. However, the algorithm provides different feature values for different scanning technologies and thus a scanner-specific approach might be needed ${ }^{121,122}$

\section{Data and Simulation Settings}

Given the above short introduction to the problem, the fingerprint data for liveness detection task was provided to GETnet as another test case for the following reasons:

1. The fingerprint is converted to two ridge signals from the first and last captures, and the decision can be considered as a corresponding bivalued target signal. This is a 2D to 1-D sequence mapping, which is an ideal form for GETnet.

2. This is a non-standard problem for which an optimal classical solution has not been offered. It is hard to find a near optimal and orthogonal feature set for a rather vague physiological phenomenon such as perspiration. Furthermore, it is possible that the observed changes are not clear enough to the human researcher for manual feature extraction. For instance, what if the knowledge about the perspiration-related pattern changes e.g. the fact that perspiration starts from moisture-saturated pores that are $0.5 \mathrm{~mm}$ apart and flows towards the drier ridge areas, did not exist? It is also 
interesting to see a general intelligent framework such as GETnet to arrive at the same kind of solutions within a fraction of the time a human expert might need.

3. As mentioned earlier, studies have shown that the perspiration detection algorithm should be customized for different capturing technologies. With given variety of scanners as well as operation conditions (climate, demographic, etc), it is more efficient to solve the problem through a general framework such as GETnet and avoid resolving manually for each setting.

The aim of this task is demonstrating the ability of GETnet in evolving appropriate compact networks for the mentioned type of data. Optimal customized solutions for each dataset requires an adequate evolutionary search on the representative training sets.
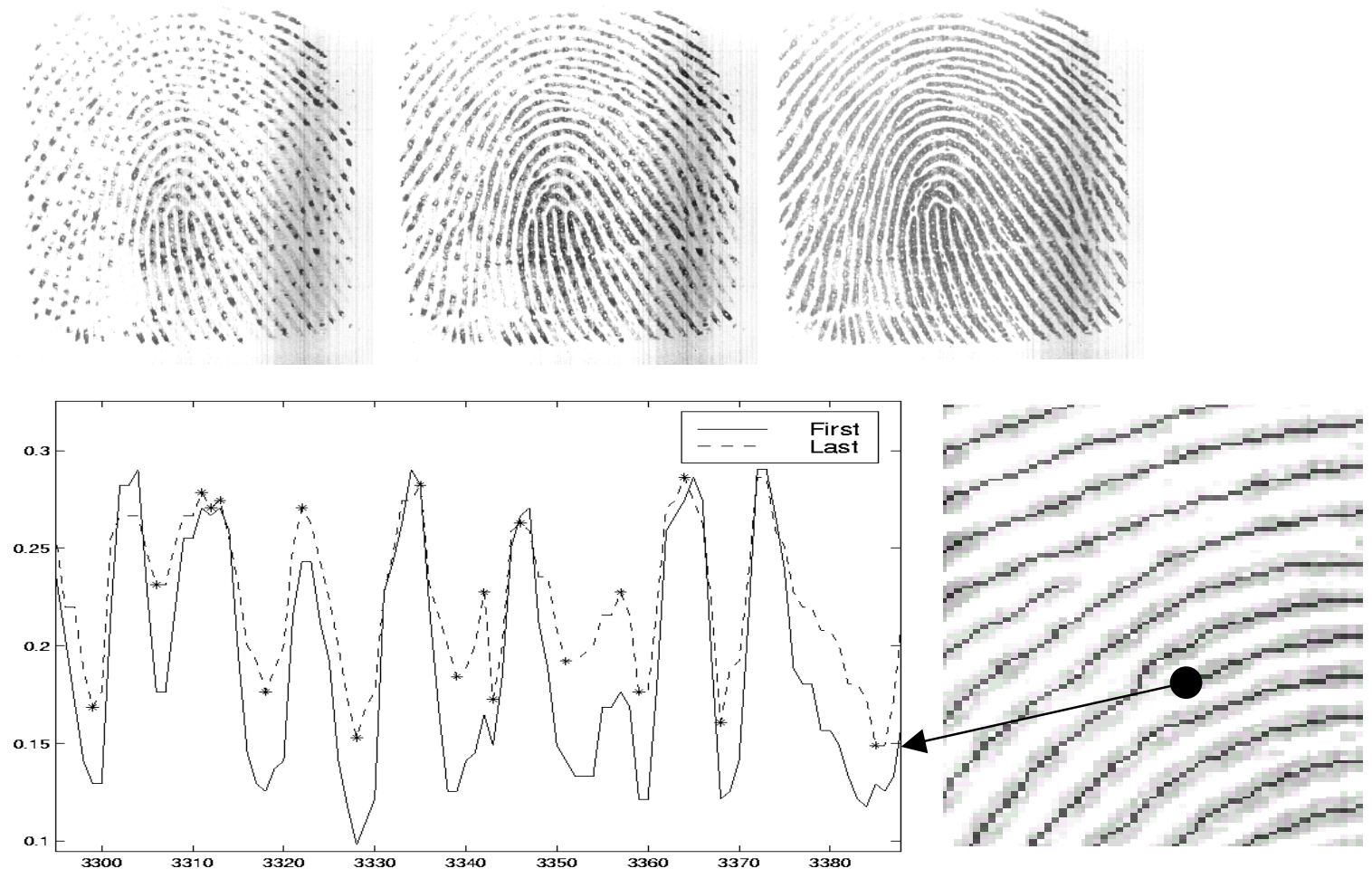

Figure 78 Perspiration-based fingerprint liveness detection. Top and from left to right: temporal progression of fingerprints. Bottom: conversion of ridge gray levels to signals. 
In order to accelerate the GETnet's evolutionary process and put into test its ability to learn from small data sets, the following settings were used:

1- Training data: 4 from spoof, 8 from live, and 4 from cadaver. Each passage only 150 samples wide.

2- Validation (early stopping) data: same composition as in training, but each passage is only 50 samples wide.

3- Test data: 10 samples from each category (live, cadaver, spoof). Full length, typically 3000 to 5000 samples wide.

4- Prune module was disabled to achieve smaller search space and thus faster evolution.

Given the nominal length of 3000 to 5000 for fingerprint ridge signals, training and early stopping data used for training and evolution (150+50 samples) constitute only 5 to 10 percent of each sample. Bipolar target signals ( -1 for non-living and +1 for live) were used. The data is the same used for the author's Master's thesis ${ }^{123}$. Please see the accompanying $\mathrm{CD}$ for more details about the data set.

\section{Results}

Below are the results obtained from running GETnet for the mentioned problem.

First, the results of the best evolved network and committee of networks after postevolution training by GetCommittee:

Best_Net_MSE_Train $=0.3774$

Committee_MSE_Train $=0.3694$

Please refer to figures 53 through 77 for and the following discussion for more details. 


\section{Connection maps:}

1- Connection maps of the original ancestor of the best-evolved network

$$
\begin{gathered}
\text { input_connect }=\left[\begin{array}{ll}
1 & 1 \\
1 & 1 \\
1 & 1 \\
1 & 1
\end{array}\right] \\
\text { layer_connect }=\left[\begin{array}{llll}
0 & 1 & 1 & 1 \\
1 & 1 & 1 & 0 \\
1 & 1 & 1 & 1 \\
1 & 1 & 1 & 0
\end{array}\right] \\
\text { output_connect }=\left[\begin{array}{l}
0 \\
1 \\
0 \\
0
\end{array}\right]
\end{gathered}
$$

2- Connection maps of the best-evolved network after 15 generations:

$$
\text { input_connect }=\left[\begin{array}{ll}
1 & 0 \\
1 & 1 \\
0 & 1 \\
1 & 0
\end{array}\right]
$$




$$
\text { layer_connect }=\left[\begin{array}{llll}
0 & 1 & 1 & 0 \\
0 & 1 & 1 & 0 \\
0 & 1 & 1 & 1 \\
1 & 1 & 0 & 0
\end{array}\right]
$$

$$
\text { output_connect }=\left[\begin{array}{l}
0 \\
1 \\
0 \\
0
\end{array}\right]
$$

General descriptors and strategy parameters:

1-Original ancestor of the best-evolved network:

size $($ total branches $)=95$

node_mutation_SD $=0.0460$

connection_mutation_SD $=0.0585$

delay_mautation_SD $=0.0816$.

Darwinian mutation' standard deviation (for Dar_inp_SD and Dar_lay_SD) $=0.0285$

Darwinian mutation' mean (for Dar_inp_SD and Dar_lay_SD) $=0.0465$

Connection weights' standard deviation $=0.2830$

Connection weights' mean $=-0.0048$

MSE validation (mean of multiple starts) $=0.9641$

Training/validation time (mean of multiple starts) $=314.5720 \mathrm{sec}$ 
2- Best evolved network, after 15 generations:

size $($ total branches $)=59$

node_mutation_SD $=0.0041$

connection_mutation_SD $=0.0230$

delay_mautation_SD $=0.0028$

Darwinian mutation' standard deviation (for Dar_inp_SD and Dar_lay_SD) $=0.0396$

Darwinian mutation' mean (for Dar_inp_SD and Dar_lay_SD) $=0.0382$

Connection weights' standard deviation $=0.3099$

Connection weights' mean $=0.0209$

Validation MSE (mean of multiple starts) $=0.3949$

Training/validation time (mean of multiple starts) $=151.5210 \mathrm{sec}$

\section{Weights}

1- Connection weights from original ancestor of the best-evolved network:

$$
\begin{aligned}
\text { input_weights } & =\left[\begin{array}{ll}
\overrightarrow{i w}_{11} & \overrightarrow{i w}_{12} \\
\overrightarrow{i w}_{21} & \overrightarrow{i w}_{22} \\
\overrightarrow{i w}_{31} & \overrightarrow{i w}_{32} \\
\overrightarrow{i w}_{41} & \overrightarrow{i w}_{42}
\end{array}\right] \\
\text { layer_weights } & =\left[\begin{array}{llll}
{[]} & \overrightarrow{l w}_{12} & \overrightarrow{l w}_{13} & \overrightarrow{l w}_{14} \\
\overrightarrow{l w}_{21} & \overrightarrow{l w}_{22} & \overrightarrow{l w}_{23} & {[]} \\
\overrightarrow{l w}_{31} & \overrightarrow{l w}_{33} & \overrightarrow{l w}_{34} \\
\overrightarrow{l w}_{41} & \overrightarrow{l w}_{42} & \overrightarrow{l w}_{43} & {[]}
\end{array}\right]
\end{aligned}
$$


with the following vector elements:

$$
\begin{aligned}
& \boldsymbol{i}_{11}=\left[\begin{array}{lllllll}
-0.2078 & -0.2955 & 0.1899 & 0.1313 & 0.3500 & 0.0942 & 0.0795
\end{array}\right] \\
& \boldsymbol{i \boldsymbol { w } _ { 2 1 }}=\left[\begin{array}{lllllll}
0.6064 & -0.1335 & -0.0257 & 0.0617 & 0.4298 & -0.1418 & 0.4980
\end{array}\right] \\
& \boldsymbol{i} \boldsymbol{w}_{31}=\left[\begin{array}{llllllll}
-0.0688 & 0.2004 & -0.2285 & 0.3354 & -0.1654 & -0.1459 & 0.1552 & -0.4115
\end{array}\right] \\
& \boldsymbol{i w}_{41}=\left[\begin{array}{lllll}
-0.5504 & 0.6774 & -0.1826 & -0.2261 & -0.3822
\end{array}\right] \\
& \boldsymbol{i} \boldsymbol{w}_{12}=\left[\begin{array}{llll}
0.3688 & -0.2549 & -0.4596 & 0.1880
\end{array}\right] \\
& \boldsymbol{i}_{22}=\left[\begin{array}{llll}
-0.2716 & -0.3174 & -0.1624 & -0.0181
\end{array}\right] \\
& i \boldsymbol{w}_{32}=\left[\begin{array}{ll}
0.0384 & 0.0748
\end{array}\right] \\
& \boldsymbol{i} \boldsymbol{w}_{42}=\left[\begin{array}{llll}
0.3145 & -0.2330 & -0.2811 & 0.1552
\end{array}\right]
\end{aligned}
$$

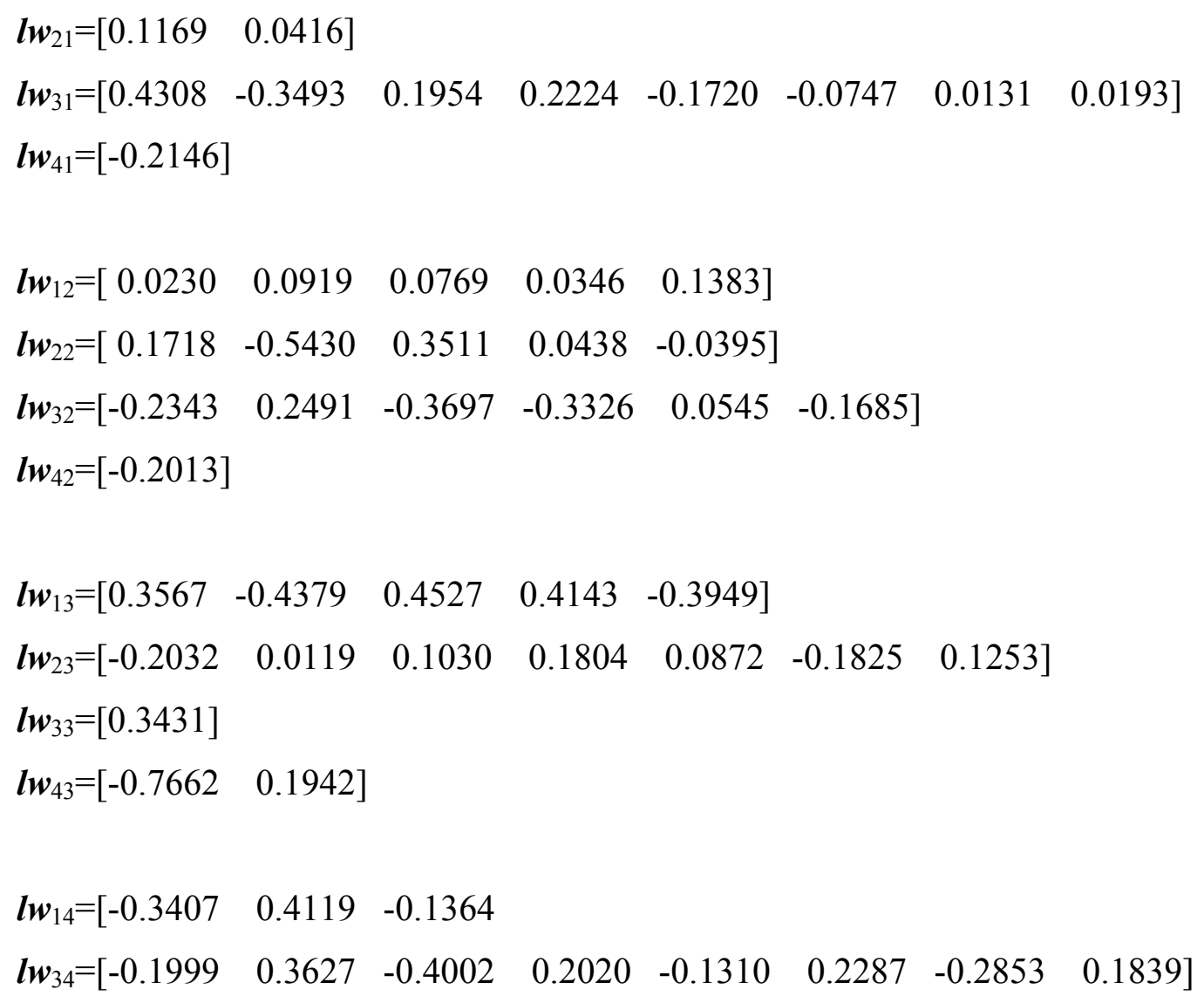


2- Connection weights, best evolved network after 15 generations:

$$
\begin{gathered}
\text { input_weights }=\left[\begin{array}{cc}
\overrightarrow{i w}_{11} & {[]} \\
\overrightarrow{i w}_{21} & \overrightarrow{i w}_{22} \\
{[]} & \overrightarrow{i w}_{32} \\
\overrightarrow{i w} 41 & {[]}
\end{array}\right] \\
\text { layer_weights }=\left[\begin{array}{cccc}
{[]} & \overrightarrow{l w}_{12} & \overrightarrow{l w}_{13} & {[]} \\
{[]} & \overrightarrow{l w}_{22} & \overrightarrow{l w}_{23} & {[]} \\
{[]} & \overrightarrow{l w}_{32} & \overrightarrow{l w}_{33} & \overrightarrow{l w_{34}} \\
\overrightarrow{l w_{41}} & \overrightarrow{l w}_{42} & {[]} & {[]}
\end{array}\right]
\end{gathered}
$$

with the following vector elements:

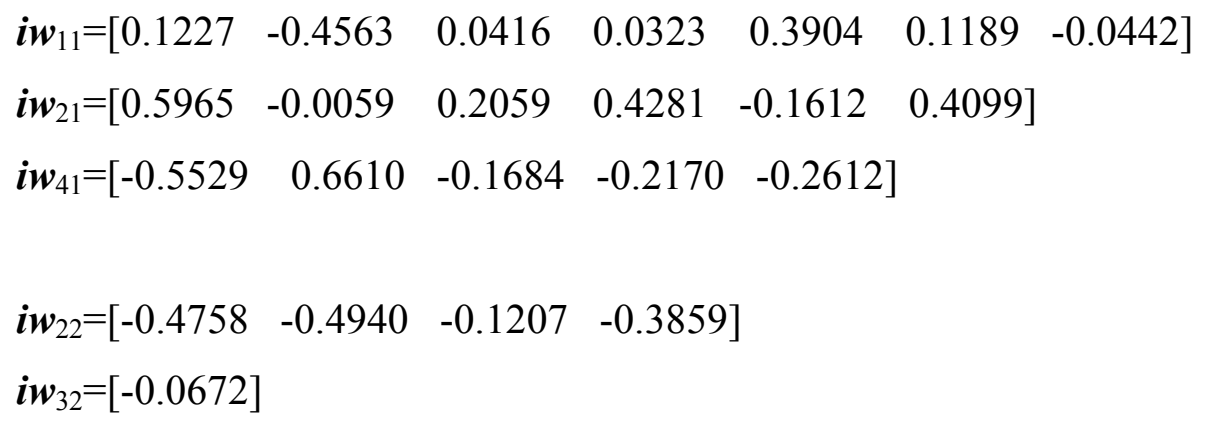




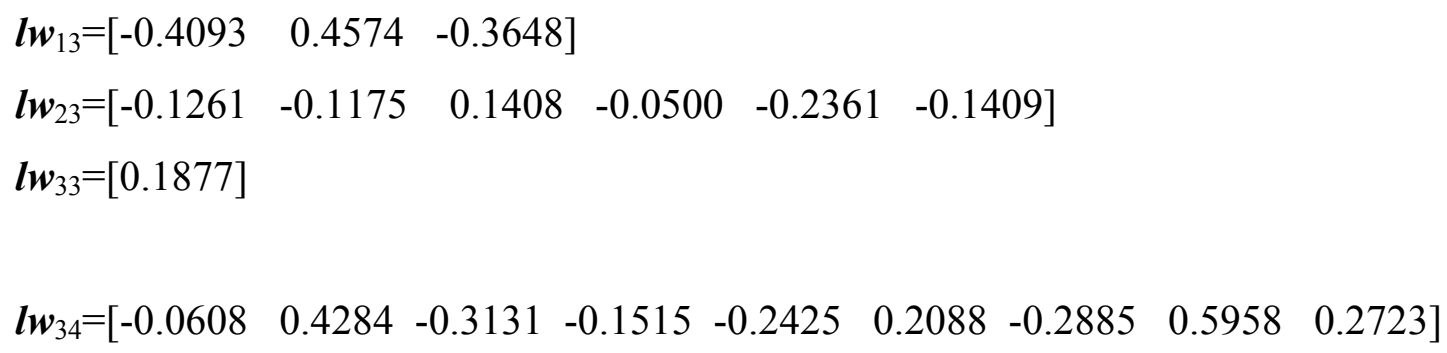

\section{Weight Evolution}

Evolutionary training of the weights is performed through Gaussian perturbations, which is determined by the standard deviation matrices.

1- Original ancestor of the best-evolved network:

$$
\begin{aligned}
& \text { Dar_inp_SD }=\left[\begin{array}{ll}
\overrightarrow{d i S D}_{11} & \overrightarrow{d i S D}_{12} \\
\overrightarrow{d i S D}_{21} & \overrightarrow{d i S D}_{22} \\
\overrightarrow{d i S D}_{31} & \overrightarrow{d i S D}_{32} \\
\overrightarrow{d i S D}_{41} & \overrightarrow{d i S D}_{42}
\end{array}\right] \\
& D_{\text {Dar_lay_SD }}=\left[\begin{array}{cccc}
{[]} & \overrightarrow{d l S D}_{12} & \overrightarrow{d l S D}_{13} & \overrightarrow{d l S D}_{14} \\
\overrightarrow{d l S D}_{21} & \overrightarrow{d l S D}_{22} & \overrightarrow{d l S D}_{23} & {[]} \\
\overrightarrow{d l S D}_{31} & \overrightarrow{d l S D}_{32} & \overrightarrow{d l S D}_{33} & \overrightarrow{d l S D}_{34} \\
\overrightarrow{d l S D}_{41} & \overrightarrow{d l S D}_{42} & \overrightarrow{d l S D}_{43} & {[]}
\end{array}\right]
\end{aligned}
$$

Starting with the following vector elements:

$$
\begin{aligned}
& \operatorname{diSD}_{11}=\left[\begin{array}{lllllll}
0.0603 & 0.0586 & 0.0919 & 0.0294 & 0.0790 & 0.0642 & 0.0483
\end{array}\right] \\
& \operatorname{diSD}_{21}=\left[\begin{array}{lllllll}
0.0011 & 0.0178 & 0.0879 & 0.0906 & 0.0009 & 0.0383 & 0.0424
\end{array}\right] \\
& \operatorname{diSD}_{31}=\left[\begin{array}{llllllll}
0.0317 & 0.0540 & 0.0006 & 0.0579 & 0.0402 & 0.0356 & 0.0238 & 0.0569
\end{array}\right] \\
& \operatorname{diSD}_{41}=\left[\begin{array}{lllll}
0.0103 & 0.0862 & 0.0093 & 0.0118 & 0.0960
\end{array}\right]
\end{aligned}
$$




$$
\begin{aligned}
& \operatorname{diSD}_{12}=\left[\begin{array}{llll}
0.0998 & 0.0205 & 0.0314 & 0.0343
\end{array}\right] \\
& \operatorname{diSD}_{22}=\left[\begin{array}{llll}
0.0060 & 0.0242 & 0.0641 & 0.0622
\end{array}\right] \\
& \operatorname{diSD}_{32}=\left[\begin{array}{llll}
0.0275 & 0.0562
\end{array}\right] \\
& \operatorname{diSD}_{42}=\left[\begin{array}{llll}
0.0156 & 0.0575 & 0.0274 & 0.0423
\end{array}\right]
\end{aligned}
$$

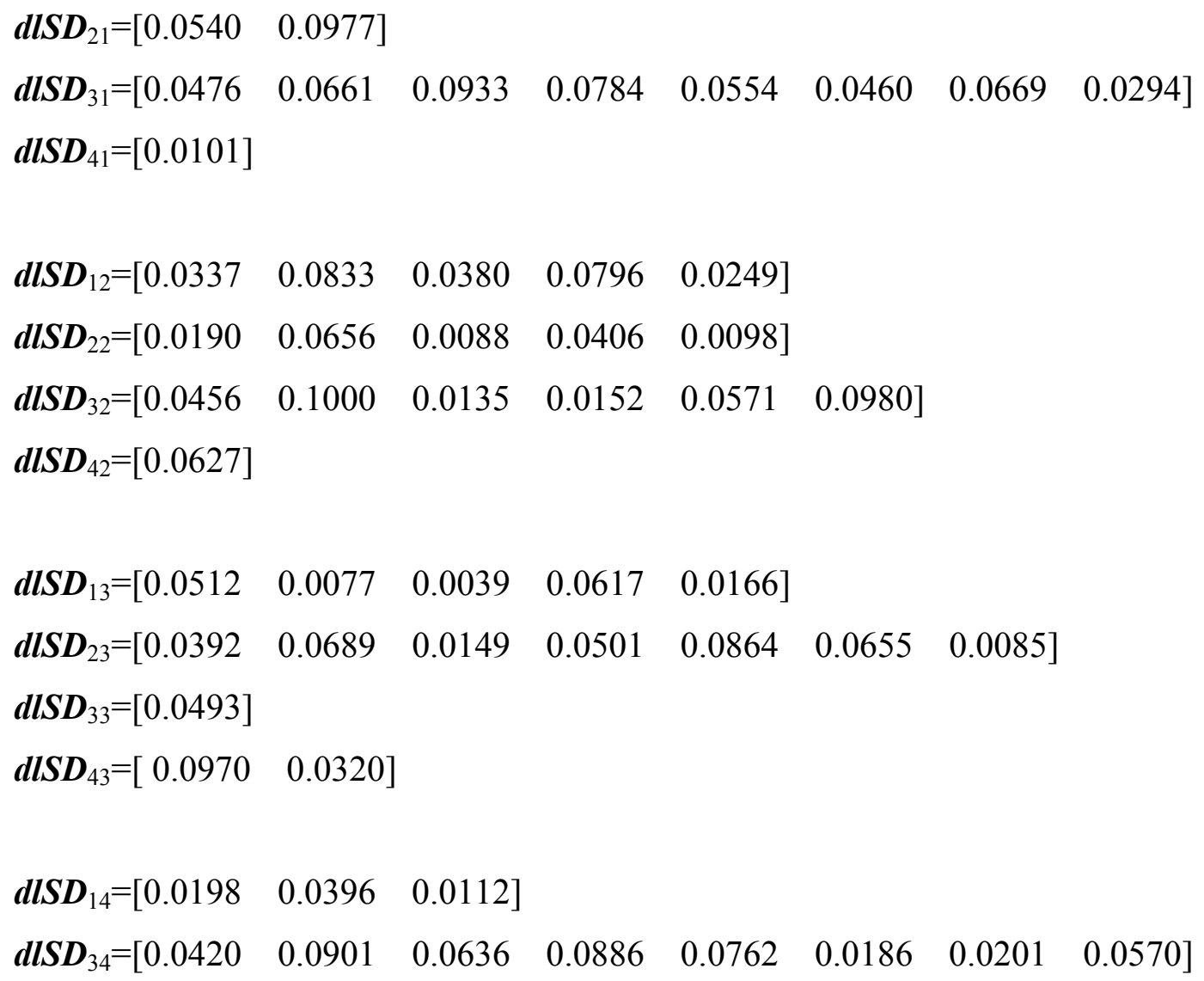

2- Standard deviation matrices of perturbation, best-evolved network:

$$
\text { Dar_inp_SD }=\left[\begin{array}{cc}
\overrightarrow{\operatorname{diSD}}_{11} & {[]} \\
\overrightarrow{\operatorname{diSD}}_{21} & \overrightarrow{\operatorname{diSD}}_{22} \\
\frac{[]}{\overrightarrow{d i S D}_{41}} & {[]}
\end{array}\right]
$$




$$
\text { Dar_lay_SD }=\left[\begin{array}{cccc}
{[]} & \overrightarrow{d l S D}_{12} & \overrightarrow{d l S D}_{13} & {[]} \\
{[]} & \overrightarrow{d l S D}_{22} & \overrightarrow{d l S D}_{23} & {[]} \\
{[]} & \overrightarrow{d l S D}_{32} & \overrightarrow{d l S D}_{33} & \overrightarrow{d l S D}_{34} \\
\overrightarrow{d l S D}_{41} & \overrightarrow{d l S D}_{42} & {[]} & {[]}
\end{array}\right]
$$

with the following vector elements:

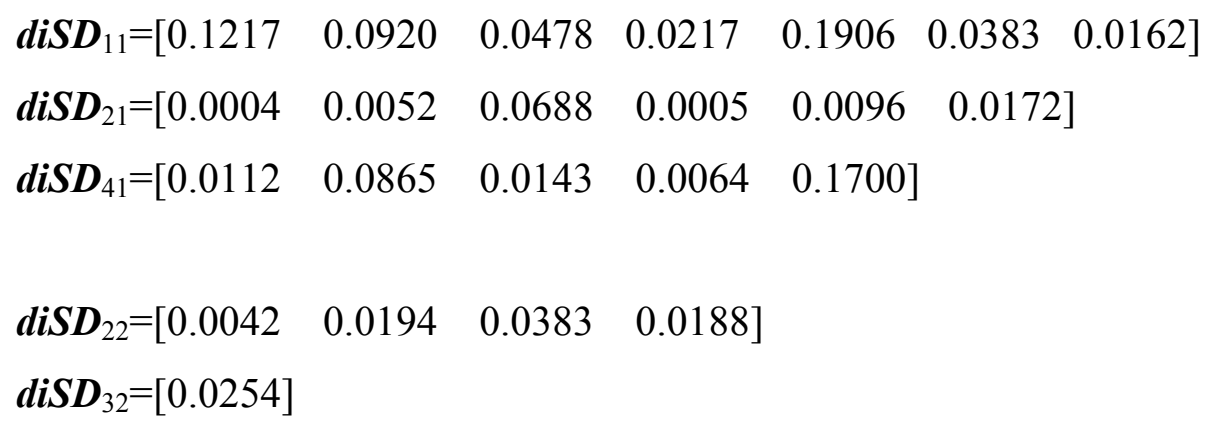




\section{Delays}

1- Branch delay matrices of the ancestor of the best-evolved network:

$$
\begin{gathered}
\text { input_delay }=\left[\begin{array}{ll}
\overrightarrow{i d}_{11} & \overrightarrow{i d}_{12} \\
\overrightarrow{i d}_{21} & \overrightarrow{i d}_{22} \\
\overrightarrow{i d}_{31} & \overrightarrow{i d}_{32} \\
\overrightarrow{i d}_{41} & \overrightarrow{i d}_{42}
\end{array}\right] \\
\text { layer_delay }=\left[\begin{array}{llll}
{[]} & \overrightarrow{l d}_{12} & \overrightarrow{l d}_{13} & \overrightarrow{l d}_{14} \\
\overrightarrow{l d}_{21} & \overrightarrow{l d}_{22} & \overrightarrow{l d}_{23} & {[]} \\
\overrightarrow{l d}_{31} & \overrightarrow{l d}_{32} & \overrightarrow{l d}_{33} & \overrightarrow{l d}_{34} \\
\overrightarrow{l d}_{41} & \overrightarrow{l d}_{42} & \overrightarrow{l d}_{43} & {[]}
\end{array}\right]
\end{gathered}
$$

Starting with the following vector elements:

$$
\begin{aligned}
& \boldsymbol{i d}_{11}=\left[\begin{array}{lllllll}
1 & 2 & 4 & 5 & 6 & 7 & 8
\end{array}\right] \\
& \boldsymbol{i d}_{21}=\left[\begin{array}{lllllll}
1 & 2 & 4 & 5 & 6 & 7 & 8
\end{array}\right] \\
& i \boldsymbol{d}_{31}=\left[\begin{array}{llllllll}
0 & 1 & 2 & 3 & 4 & 5 & 7 & 8
\end{array}\right] \\
& \boldsymbol{i d}_{41}=\left[\begin{array}{lllll}
0 & 1 & 3 & 4 & 5
\end{array}\right]
\end{aligned}
$$

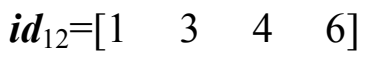

$$
\begin{aligned}
& i \boldsymbol{d}_{22}=\left[\begin{array}{lllll}
2 & 3 & 4 & 5 & 6
\end{array}\right] \\
& \boldsymbol{i d}_{32}=\left[\begin{array}{ll}
1 & 4
\end{array}\right] \\
& \boldsymbol{i} \boldsymbol{d}_{42}=\left[\begin{array}{llll}
2 & 3 & 5 & 6
\end{array}\right]
\end{aligned}
$$

$$
\begin{aligned}
& \boldsymbol{l} \boldsymbol{d}_{21}=\left[\begin{array}{ll}
1 & 5
\end{array}\right] \\
& \boldsymbol{l} \boldsymbol{d}_{31}=\left[\begin{array}{llllllll}
0 & 1 & 2 & 3 & 4 & 5 & 6 & 8
\end{array}\right] \\
& \boldsymbol{l d}_{41}=[4]
\end{aligned}
$$




$$
\begin{aligned}
& \boldsymbol{l d}_{12}=\left[\begin{array}{lllll}
1 & 2 & 6 & 7 & 8
\end{array}\right] \\
& \boldsymbol{l d} \boldsymbol{d}_{22}=\left[\begin{array}{llll}
2 & 3 & 4 & 6
\end{array}\right] \\
& \boldsymbol{l} \boldsymbol{d}_{32}=\left[\begin{array}{llllll}
1 & 2 & 3 & 4 & 6 & 7
\end{array}\right] \\
& \boldsymbol{l d}_{42}=[1] \\
& \boldsymbol{l d}_{13}=\left[\begin{array}{lllll}
1 & 5 & 6 & 7 & 8
\end{array}\right] \\
& \boldsymbol{l d}_{23}=\left[\begin{array}{lllllll}
3 & 4 & 5 & 6 & 7 & 8 & 9
\end{array}\right] \\
& \boldsymbol{l d}_{33}=\text { [1] } \\
& \boldsymbol{l d} \boldsymbol{d}_{43}=\left[\begin{array}{ll}
3 & 4
\end{array}\right] \\
& \boldsymbol{l d}_{14}=\left[\begin{array}{lll}
1 & 5 & 6
\end{array}\right] \\
& \boldsymbol{l} \boldsymbol{d}_{34}=\left[\begin{array}{llllllll}
1 & 2 & 3 & 4 & 5 & 6 & 7 & 8
\end{array}\right]
\end{aligned}
$$

2- Branch delays, best evolved network:

$$
\begin{gathered}
\text { input_delay }=\left[\begin{array}{cc}
\overrightarrow{i d}_{11} & {[]} \\
\overrightarrow{i d}_{21} & \overrightarrow{i d}_{22} \\
{[]} & \overrightarrow{i d}_{32} \\
\overrightarrow{i d}_{41} & {[]}
\end{array}\right] \\
\text { layer_delay }=\left[\begin{array}{cccc}
{[]} & \overrightarrow{l d}_{12} & \overrightarrow{l d}_{13} & {[]} \\
{[]} & \overrightarrow{l d}_{22} & \overrightarrow{l d}_{23} & {[]} \\
{[]} & \overrightarrow{l d}_{32} & \overrightarrow{l d}_{33} & \overrightarrow{l d}_{34} \\
\overrightarrow{l d}_{41} & \overrightarrow{l d}_{42} & {[]} & {[]}
\end{array}\right]
\end{gathered}
$$

with the following vector elements:

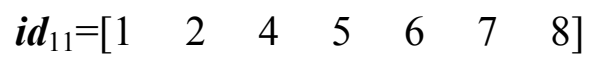




\begin{tabular}{|c|c|c|c|}
\hline $\boldsymbol{i d}_{21}=[1$ & 2 & 4 & 6 \\
\hline $\boldsymbol{i d}_{41}=[0$ & 1 & 3 & 4 \\
\hline $\boldsymbol{i d}_{22}=[0$ & 4 & 5 & 7] \\
\hline
\end{tabular}

$\boldsymbol{l d}_{41}=[4]$

$\boldsymbol{l d}_{12}=\left[\begin{array}{llllll}1 & 2 & 6 & 7 & 8 & 12\end{array}\right]$

$\boldsymbol{l d}_{22}=\left[\begin{array}{llll}2 & 3 & 4 & 6\end{array}\right]$

$\boldsymbol{l d}_{32}=\left[\begin{array}{lllll}1 & 2 & 4 & 6 & 7\end{array}\right]$

$\operatorname{ld}_{42}=[1]$

$\boldsymbol{l d}_{13}=\left[\begin{array}{lll}5 & 6 & 8\end{array}\right]$

$\boldsymbol{l d}_{23}=\left[\begin{array}{llllll}4 & 5 & 6 & 7 & 8 & 9\end{array}\right]$

ld $_{33}=[1]$

$\boldsymbol{l d}_{34}=\left[\begin{array}{lllllllll}1 & 2 & 3 & 4 & 5 & 6 & 7 & 8 & 12\end{array}\right]$

A summary of the results is given through the following tables. As it can be seen, 3 live specimens were falsely recognized as nonliving, whereas one out of 10 for each spoof and cadaver test data sets were falsely recognized as live. The overall precision is therefore $(30-3-1-1) / 30=83.3 \%$. The output values in the tables are calculated as the net area under output curve

$$
\text { Output }_{i}=\int_{\text {ridge_signal }} y_{i}(\tau) d \tau
$$

For discrete outputs of the GETnet program, (C27) is simply evaluated as a summation of the output array. 
The liveness results are determined as

$$
\text { Liveness } \left._{i}=\text { hard_threshold }_{\text {Output }}{ }_{i}\right)
$$

Hard limiting threshold function (see B40) returns -1 for nonliving and 1 for living as the final classification result. The final evolved network is depicted below.

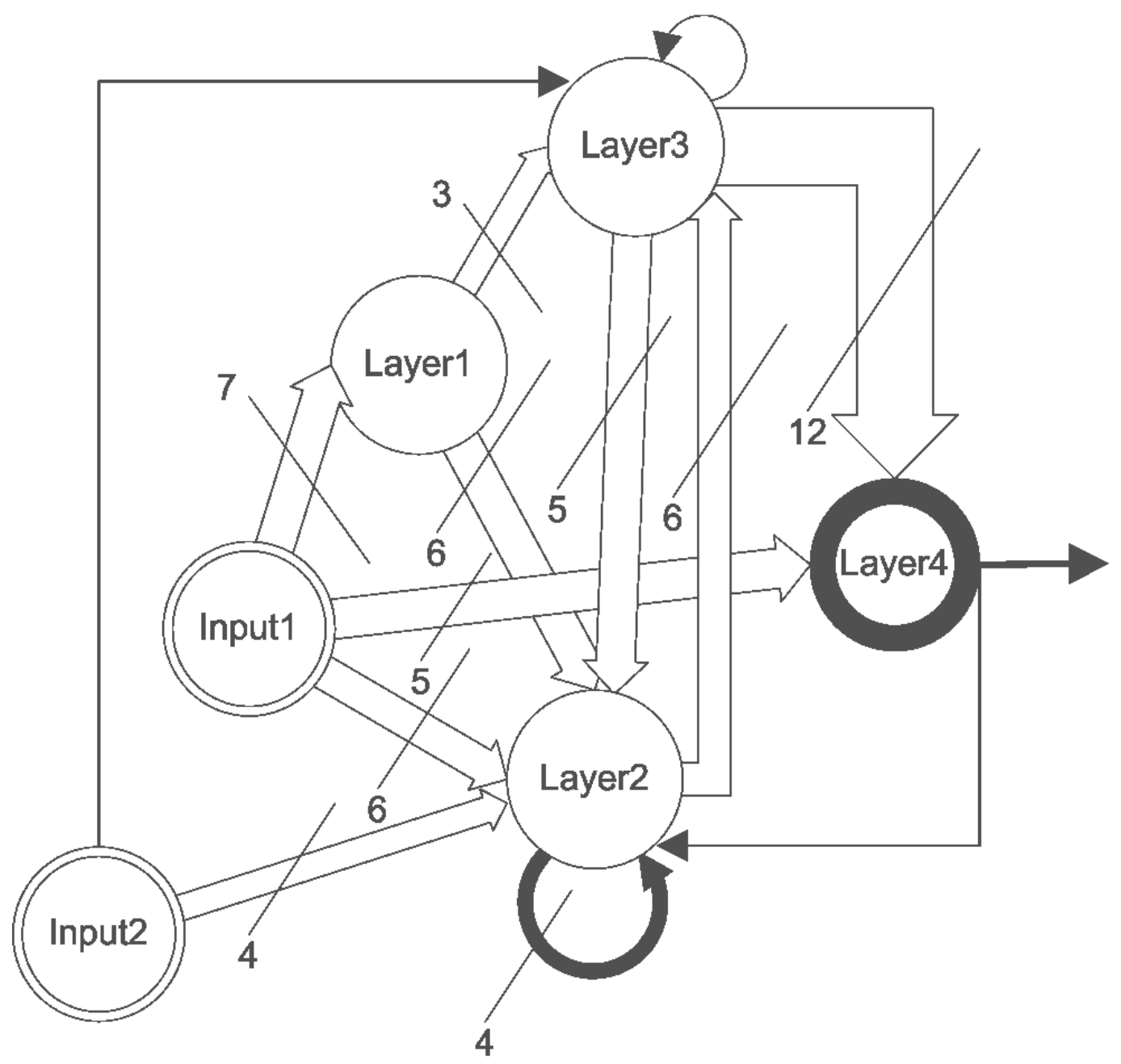

Figure 79 Best evolved network for fingerprint liveness detection. Note the novel structure, delayed weight bus widths, and multiple feedback loops. 
Table 2 Test outputs for live subjects. Incorrect classifications are italicized.

\begin{tabular}{|c|c|c|c|}
\hline Subject & Best Net Output & Committee Output & Liveness \\
\hline LivTst1 & 0.7596 & 0.7701 & 1 \\
\hline LivTst2 & 0.8251 & 0.8332 & 1 \\
\hline LivTst3 & 0.6708 & 0.6771 & 1 \\
\hline LivTst4 & 0.2159 & 0.3300 & 1 \\
\hline LivTst5 & 0.6020 & 0.6617 & 1 \\
\hline LivTst6 & 0.7852 & 0.7893 & 1 \\
\hline LivTst7 & -0.6284 & -0.5962 & -1 \\
\hline LivTst8 & -0.2923 & -0.2121 & -1 \\
\hline LivTst9 & -0.4239 & -0.3580 & -1 \\
\hline LivTst10 & 0.5883 & 0.5769 & 1 \\
\hline
\end{tabular}

Table 3 Test outputs for cadaver subjects. Incorrect classifications are italicized.

\begin{tabular}{|c|c|c|c|}
\hline Subject & Best Net Output & Committee Output & Liveness \\
\hline CdvTst1 & -0.6553 & -0.6383 & -1 \\
\hline CdvTst2 & 0.6934 & 0.7149 & 1 \\
\hline CdvTst3 & -0.1882 & -0.0134 & -1 \\
\hline CdvTst4 & -0.1677 & -0.1583 & -1 \\
\hline CdvTst5 & -0.7048 & -0.6803 & -1 \\
\hline CdvTst6 & -0.4266 & -0.3572 & -1 \\
\hline CdvTst7 & -0.6014 & -0.5773 & -1 \\
\hline CdvTst8 & -0.6168 & -0.5845 & -1 \\
\hline CdvTst9 & -0.6919 & -0.6822 & -1 \\
\hline CdvTst10 & -0.5695 & -0.5621 & -1 \\
\hline
\end{tabular}

Table 4 Test outputs for spoof subjects. Incorrect classifications are italicized.

\begin{tabular}{|c|c|c|c|}
\hline Subject & Best Net Output & Committee Output & Liveness \\
\hline SpfTst1 & -0.5984 & -0.5494 & -1 \\
\hline SpfTst2 & -0.6311 & -0.6057 & -1 \\
\hline SpfTst3 & -0.6563 & -0.6423 & -1 \\
\hline SpfTst4 & -0.6493 & -0.6250 & -1 \\
\hline SpfTst5 & -0.3486 & -0.1599 & -1 \\
\hline SpfTst6 & -0.0479 & -0.1518 & -1 \\
\hline SpfTst7 & 0.0229 & 0.0309 & 1 \\
\hline SpfTst8 & -0.2592 & -0.3232 & -1 \\
\hline SpfTst9 & -0.4689 & -0.4283 & -1 \\
\hline SpfTst10 & -0.2772 & -0.2025 & -1 \\
\hline
\end{tabular}


Table 5 Confusion matrix for the test data. Threshold for network output is set at zero.

\begin{tabular}{|c|c|c|c|}
\hline Actual & Live & Non-living & Total Actual \\
\hline Live & $\begin{array}{c}\mathrm{C}_{11}=7 \\
\text { Correct }\end{array}$ & $\begin{array}{c}\mathrm{C}_{12}=3 \\
\text { Misidentify }\end{array}$ & $\mathrm{C}_{11}+\mathrm{C}_{12}=10$ \\
\hline Non-living & $\begin{array}{c}\mathrm{C}_{21}=2 \\
\text { Misidentify }\end{array}$ & $\begin{array}{c}\mathrm{C}_{22}=18 \\
\mathrm{Correct}\end{array}$ & $\mathrm{C}_{21}+\mathrm{C}_{22}=20$ \\
\hline $\begin{array}{c}\text { Total Neural } \\
\text { Network }\end{array}$ & $\mathrm{C}_{11}+\mathrm{C}_{21}=9$ & $\mathrm{C}_{12}+\mathrm{C}_{22}=21$ & $\begin{array}{c}\text { Total } \\
\text { Samples }=\mathbf{3 0}\end{array}$ \\
\hline
\end{tabular}

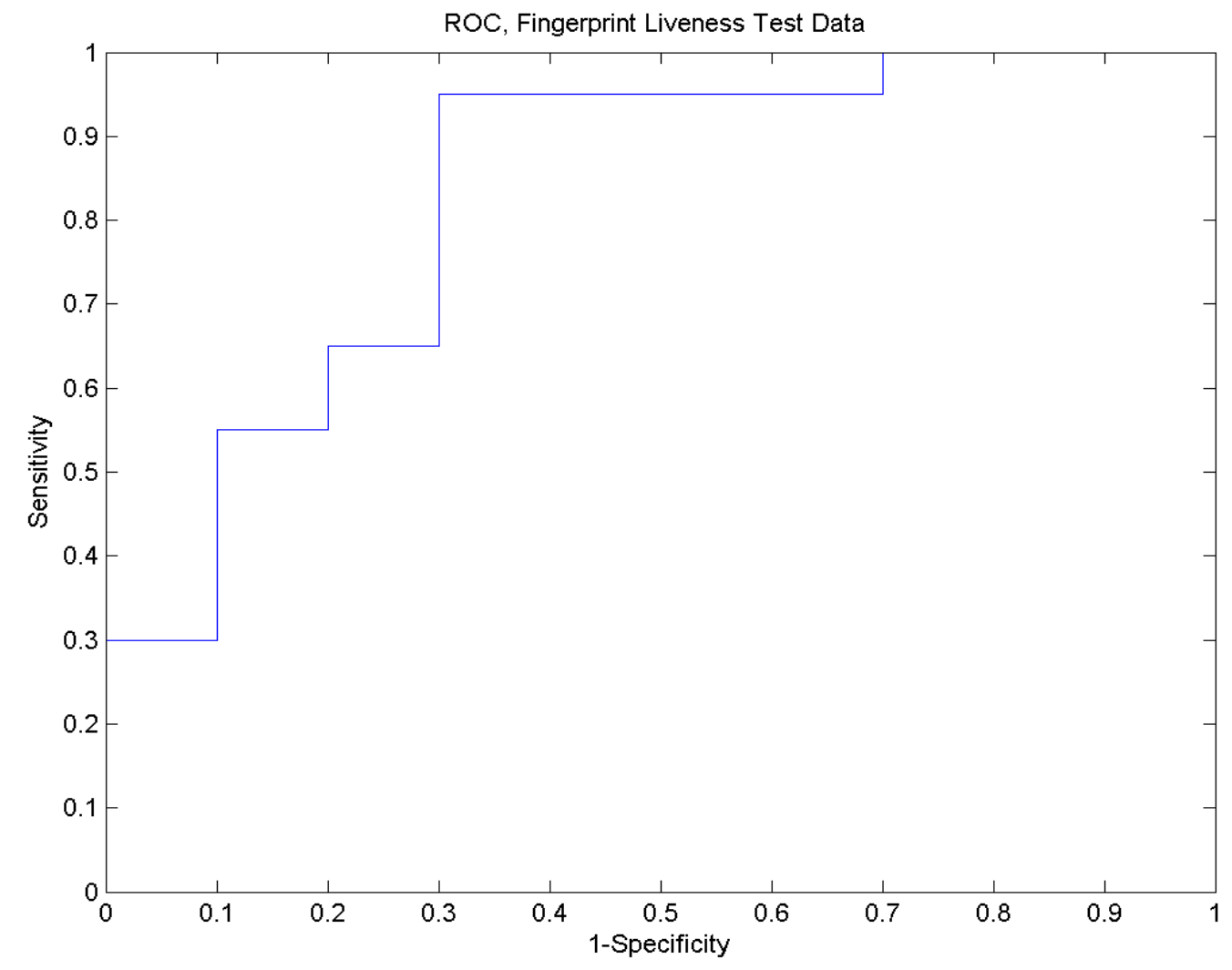

Figure 80 ROC curve for the 30 point test data. 


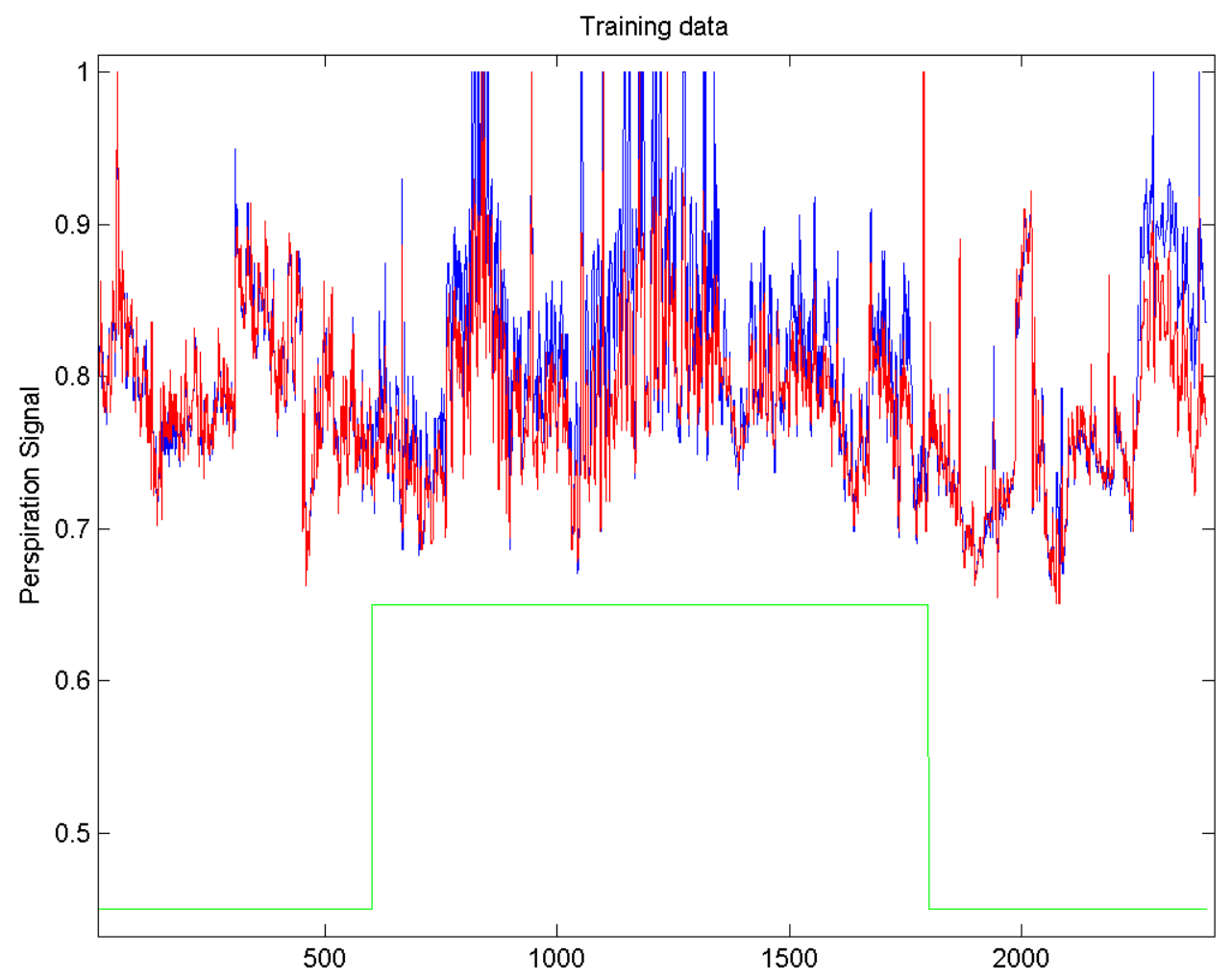

Figure 81 Training data. Red: first capture signal, blue: last capture signal. Green high: live signals, green low: nonliving signals.

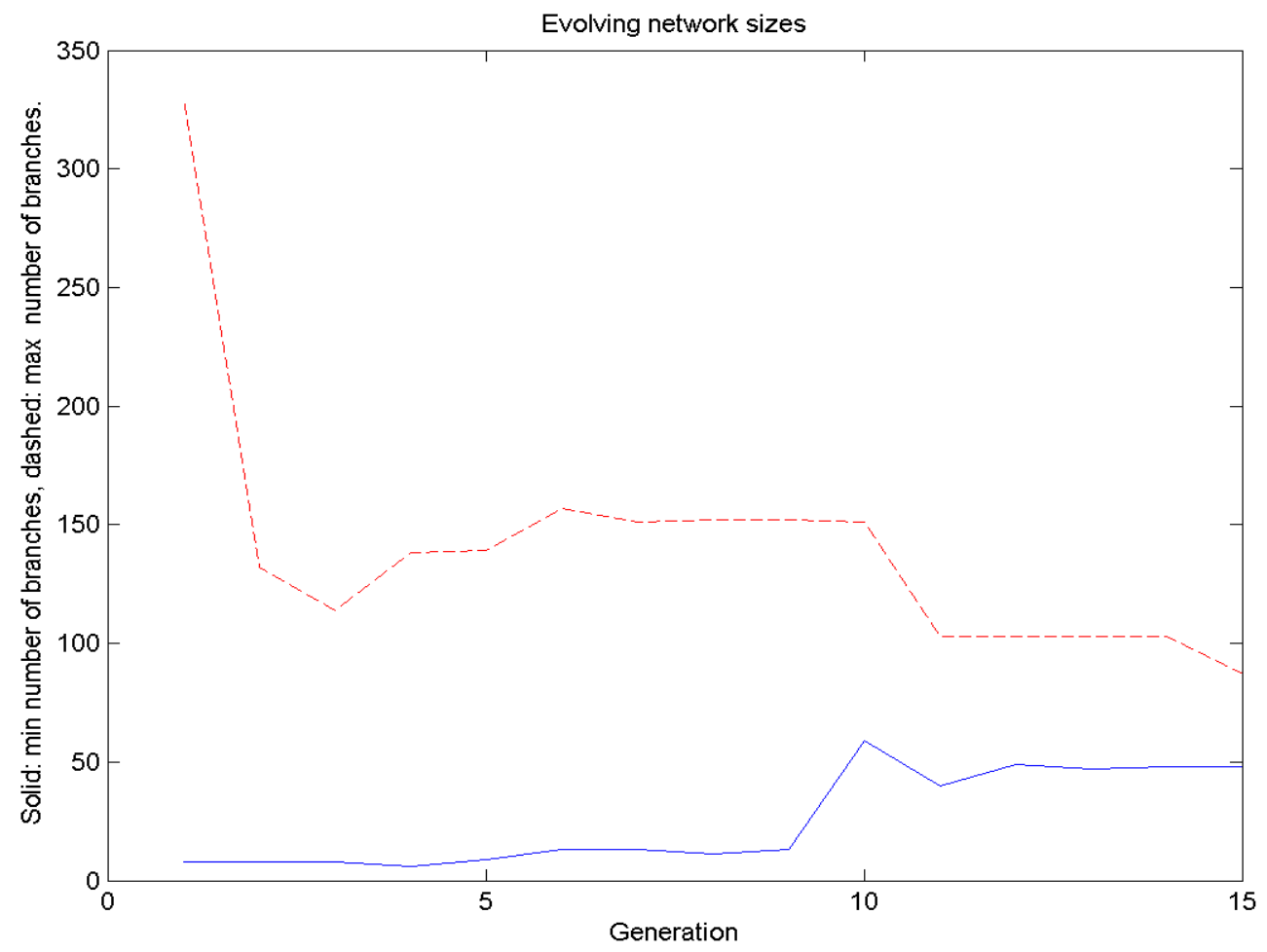

Figure 82 Size of evolving networks. 


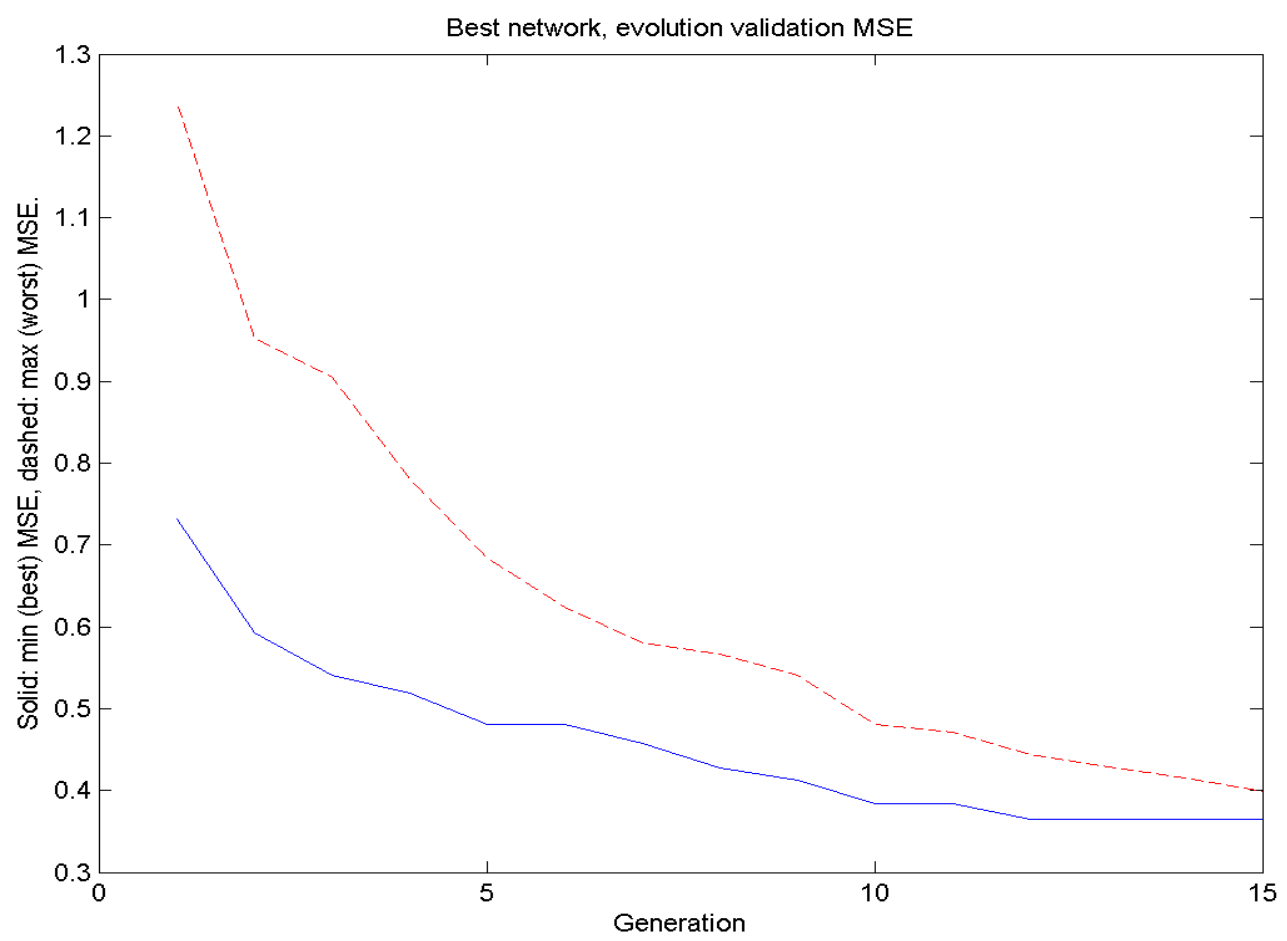

Figure 83 MSE of evolving networks.

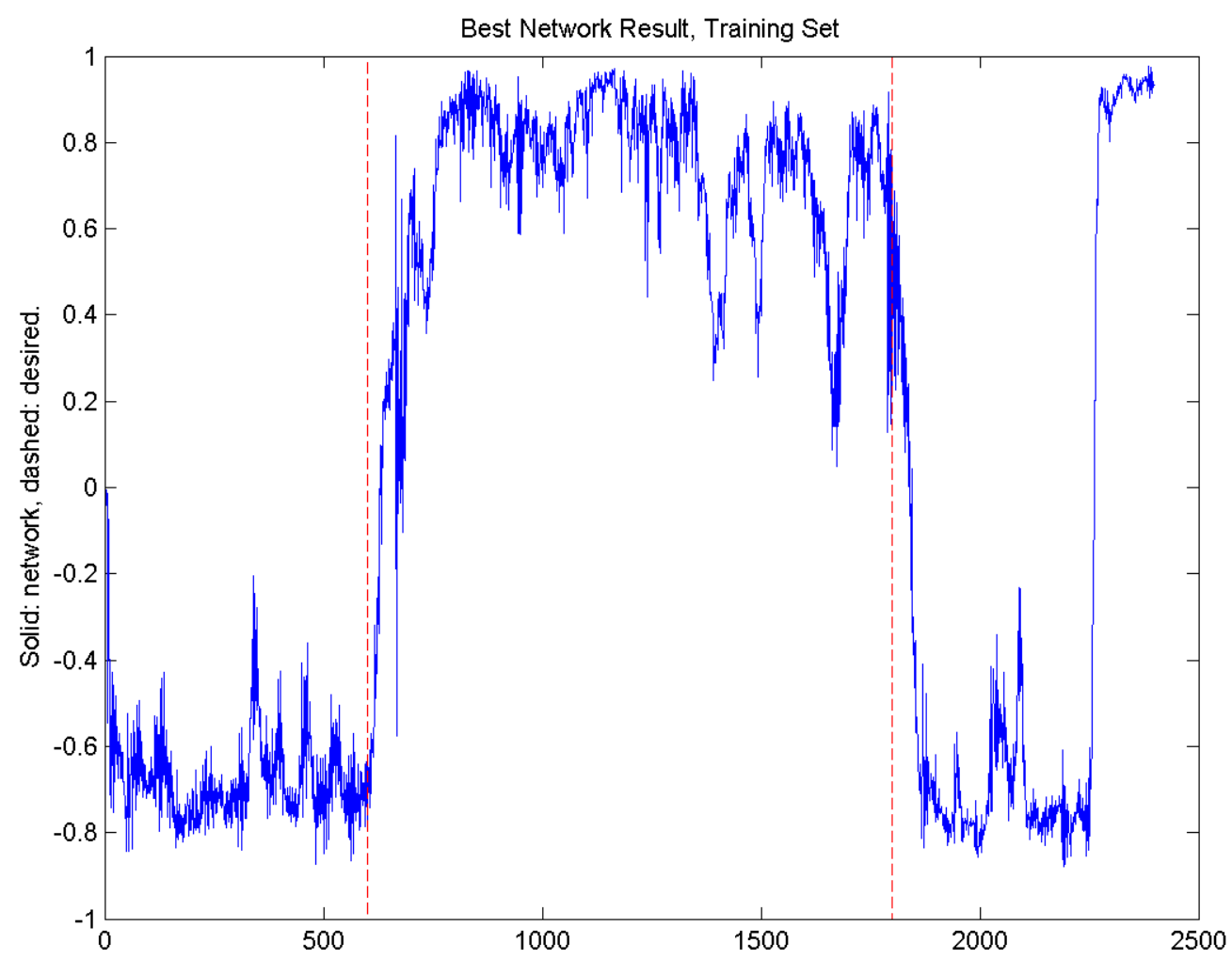

Figure 84 Training output, best evolved network. 


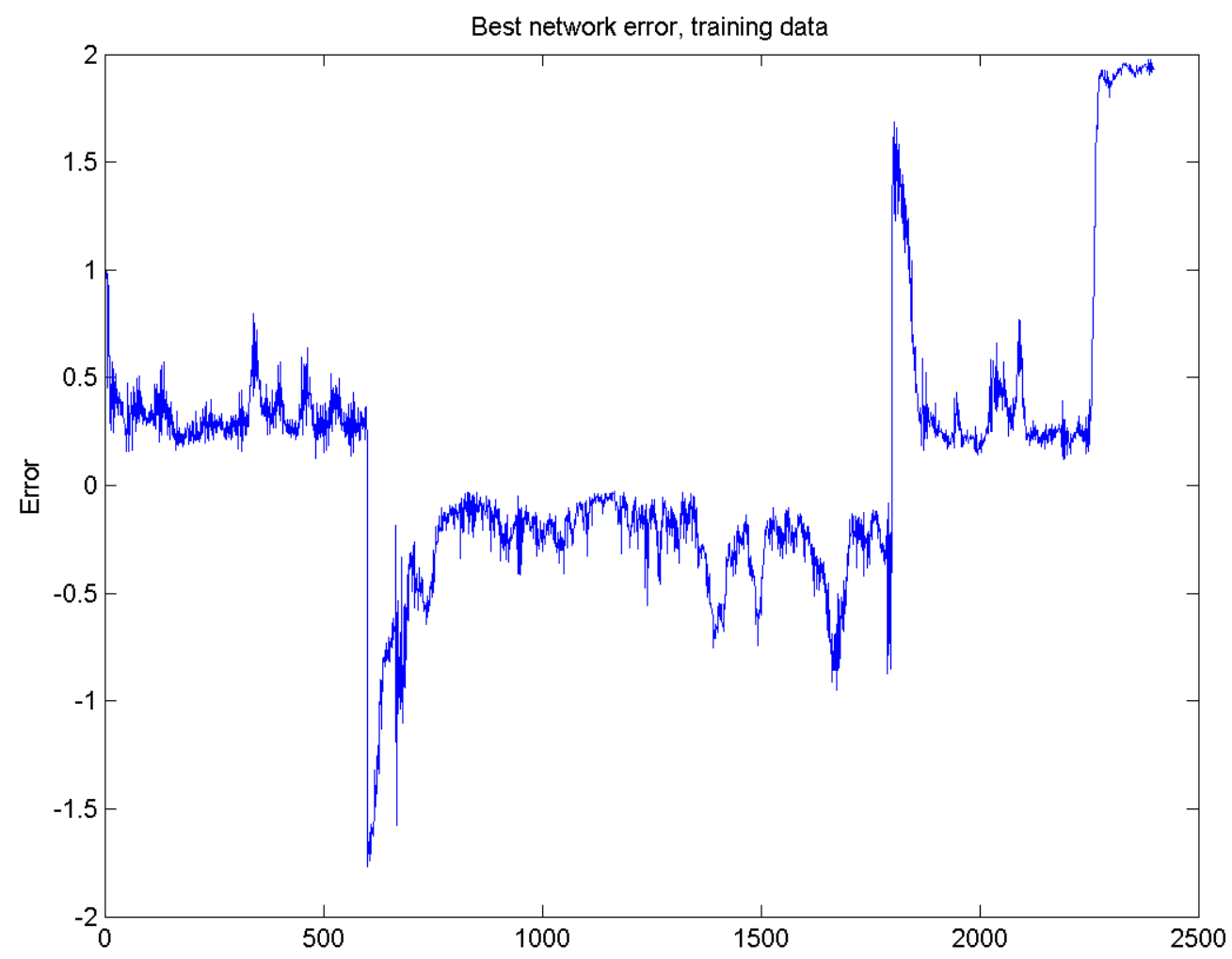

Figure 85 Training error, best network.

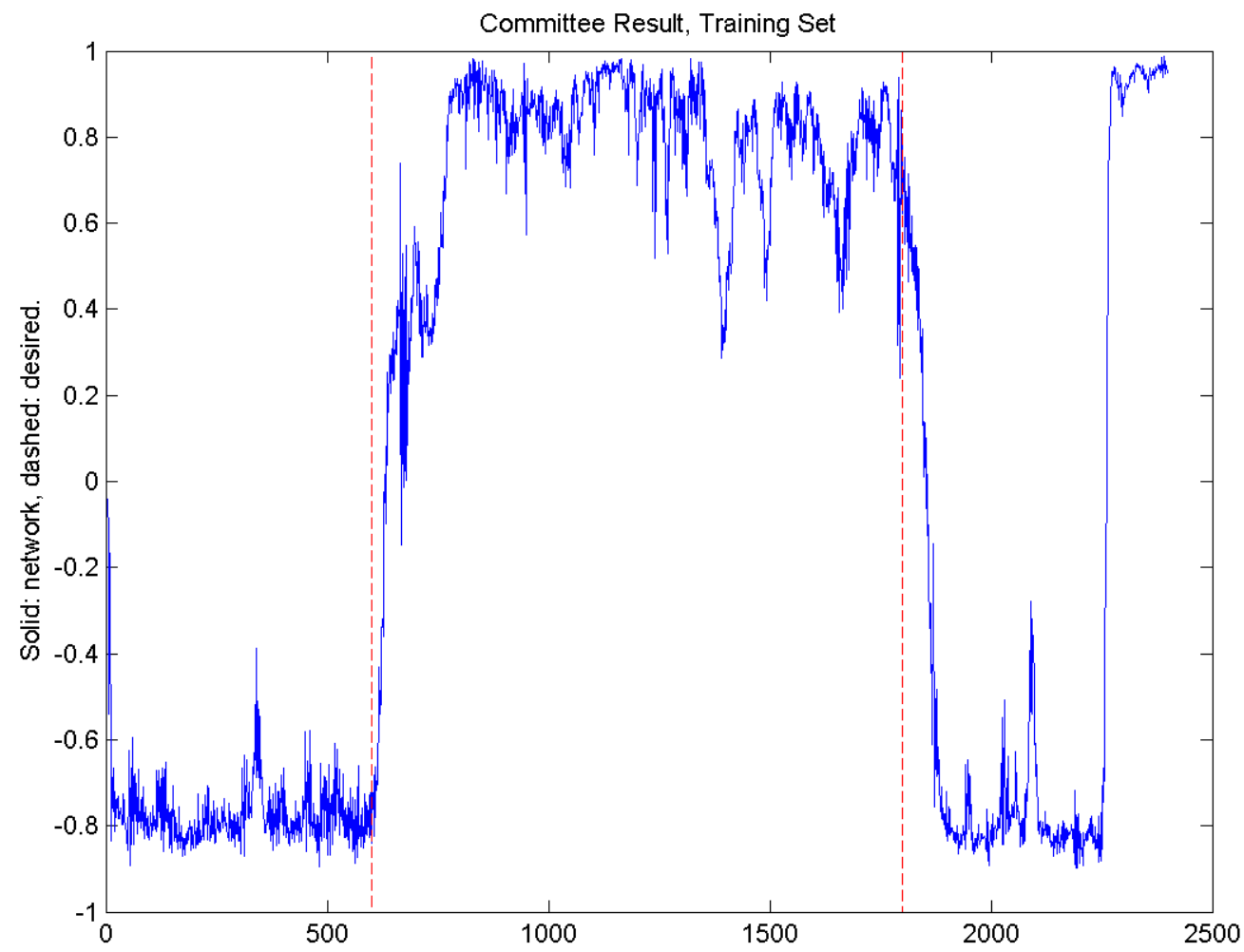

Figure 86 Training data, committee of last generation networks. 


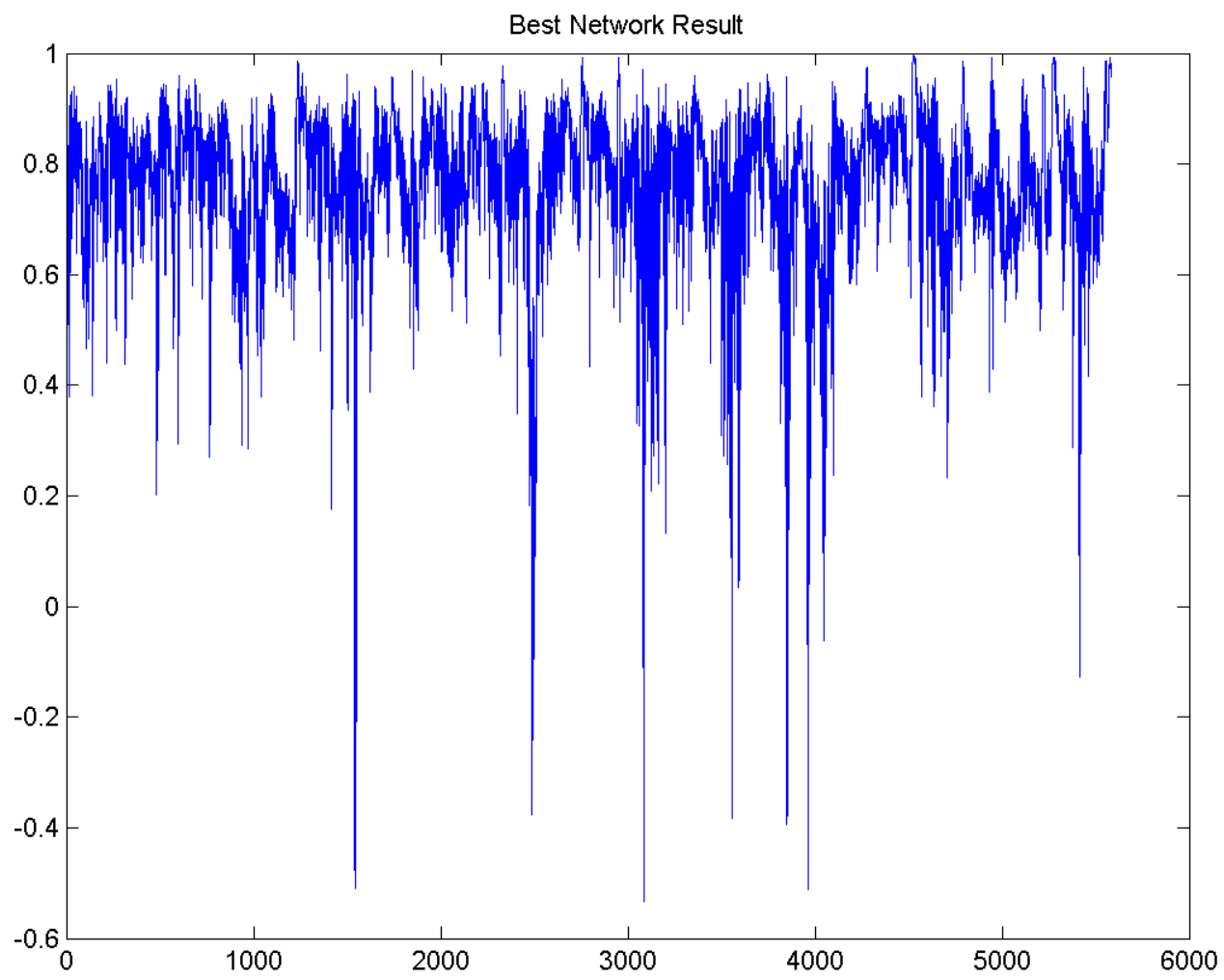

Figure 87 Sample live test data output, best evolved network.

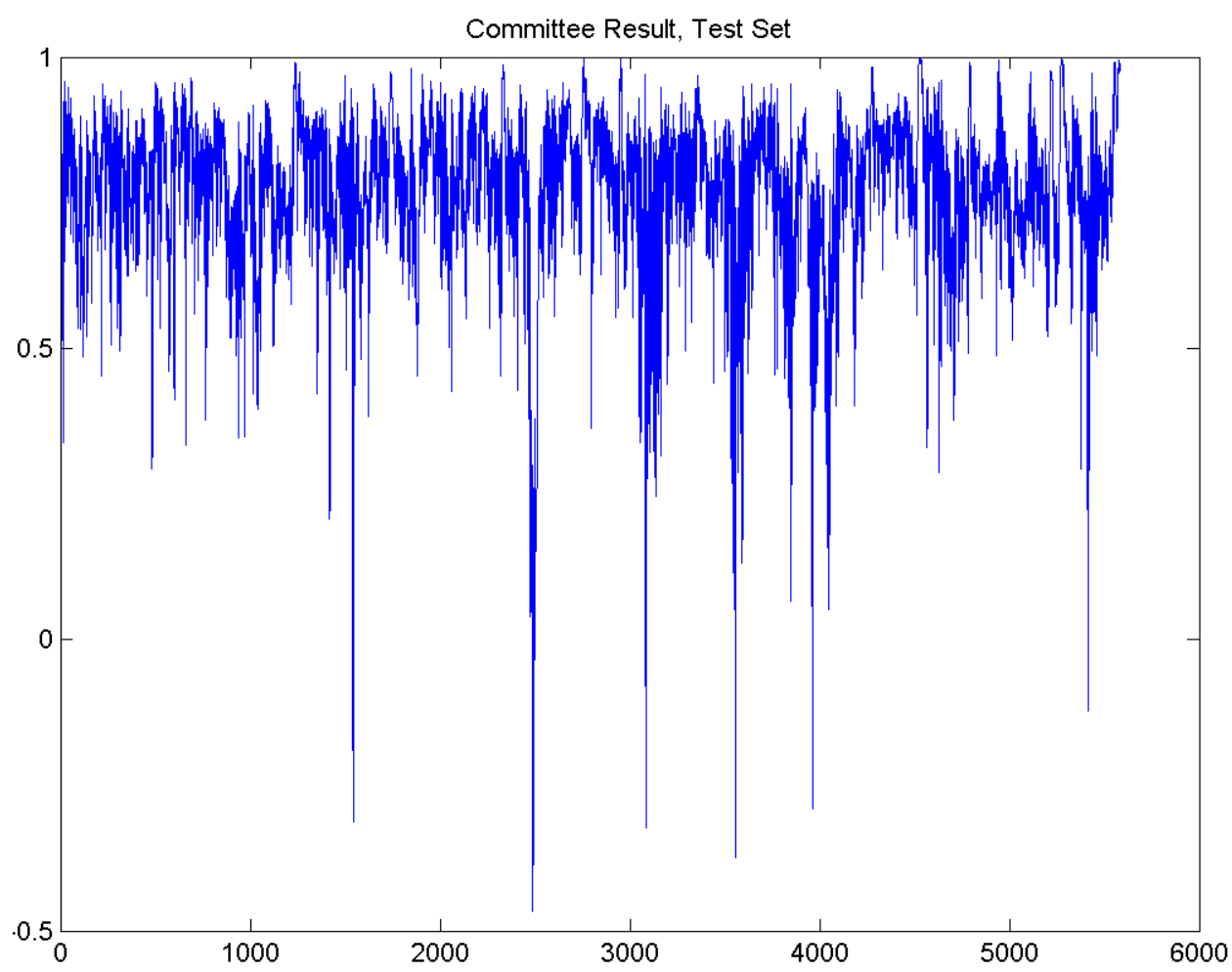

Figure 88 Sample live test data output, committee of last generation networks. 


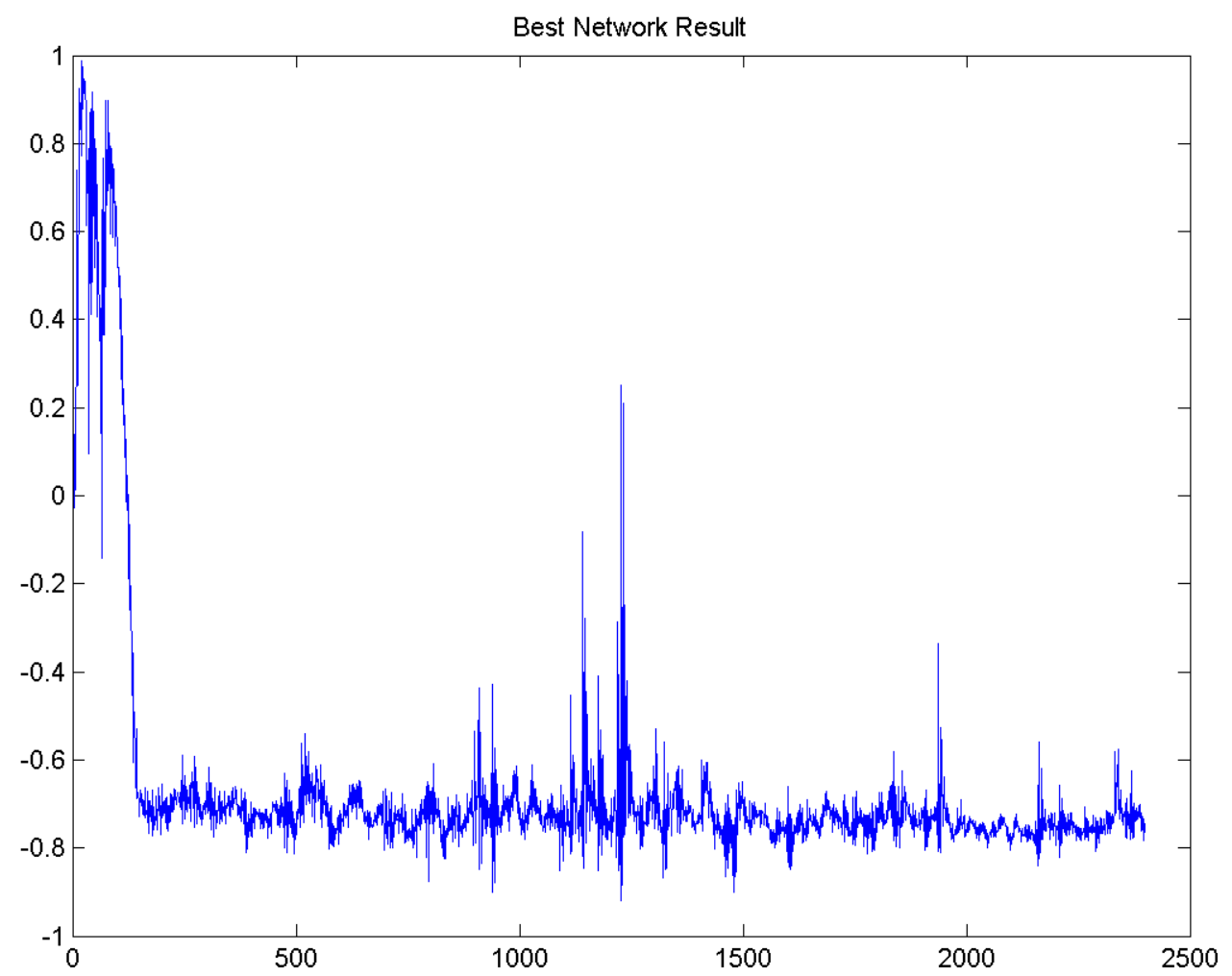

Figure 89 Sample cadaver test data output, best evolved network.

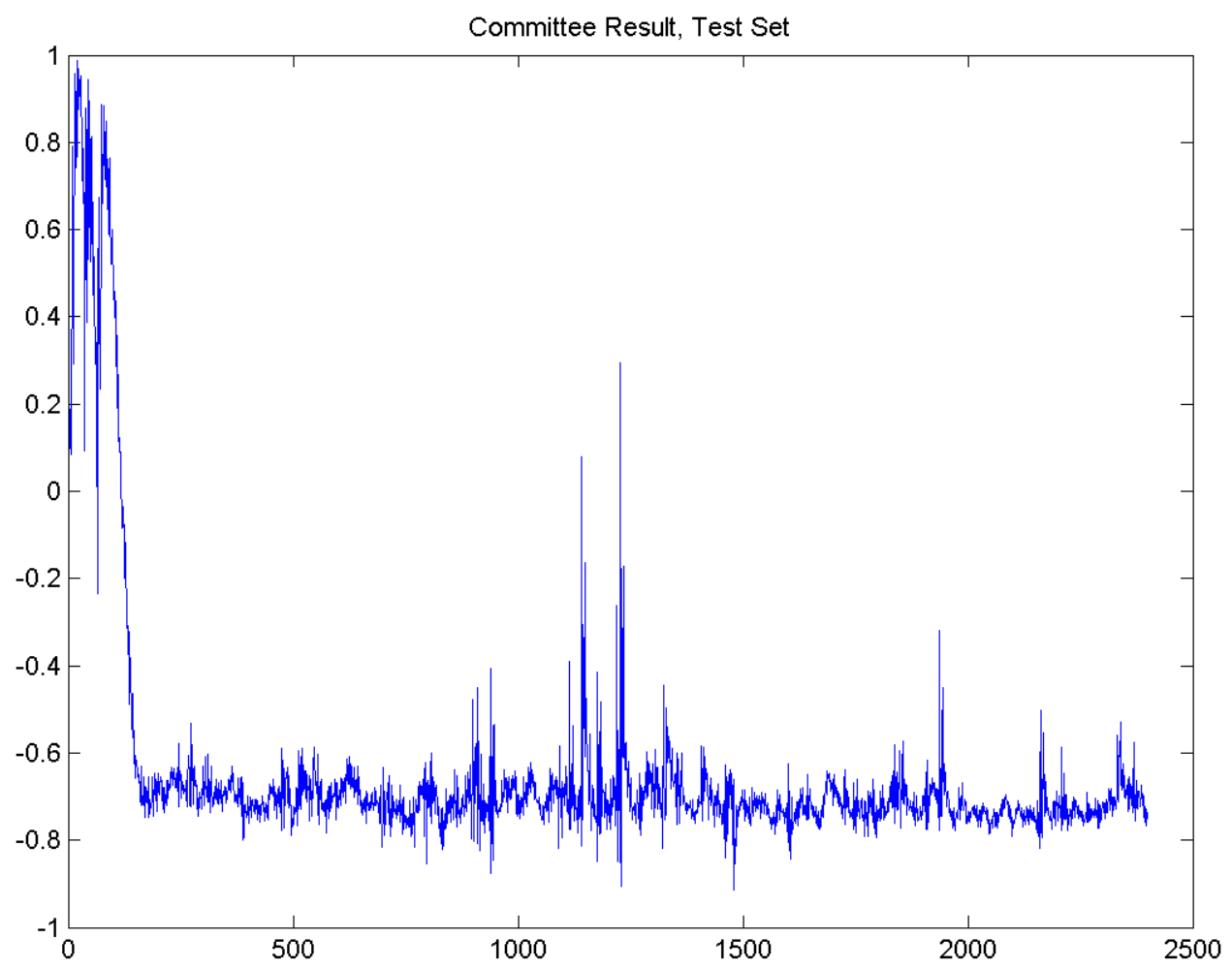

Figure 90 Sample cadaver test data output, committee of last generation networks. 


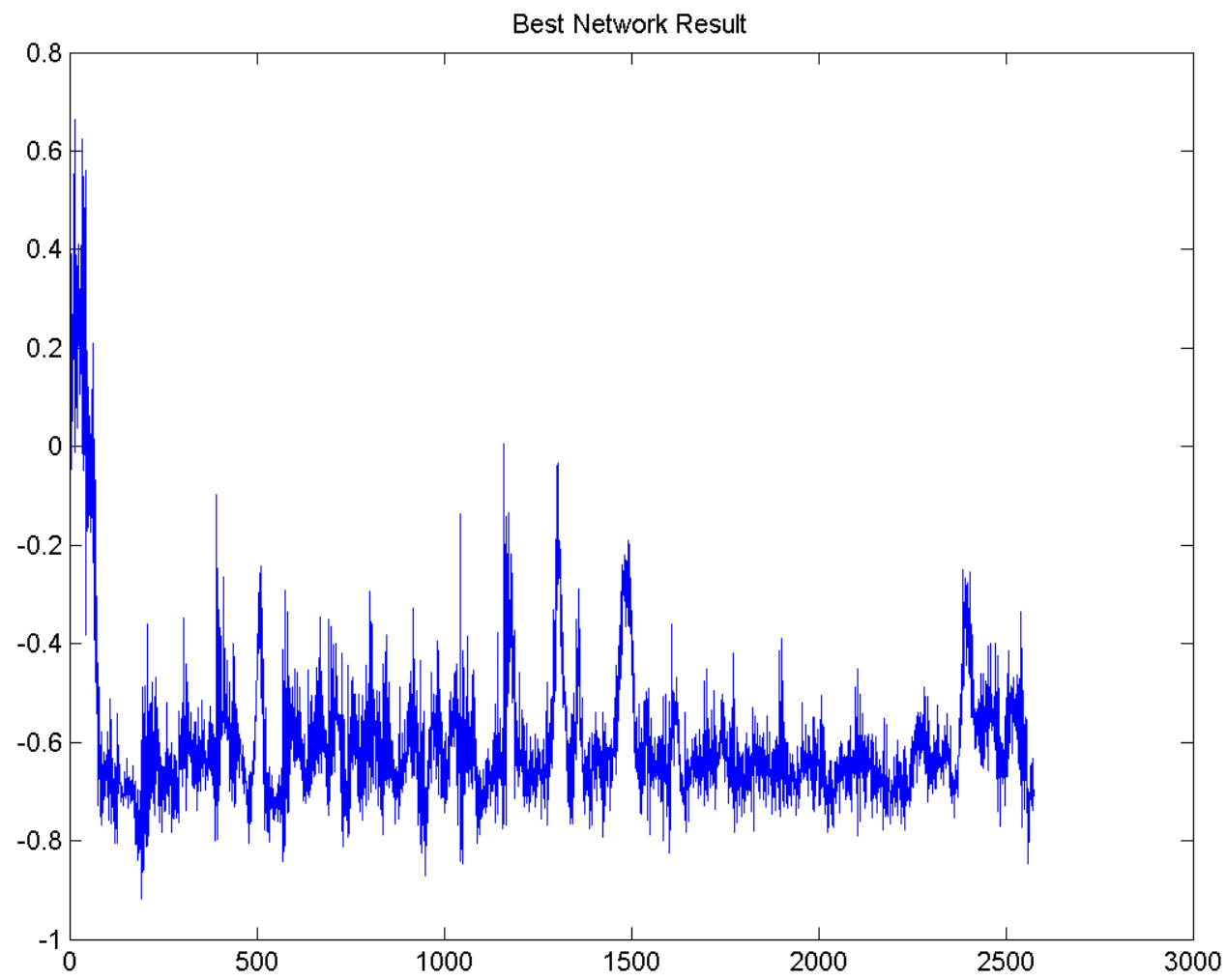

Figure 91 Sample spoof test data output, best evolved network.

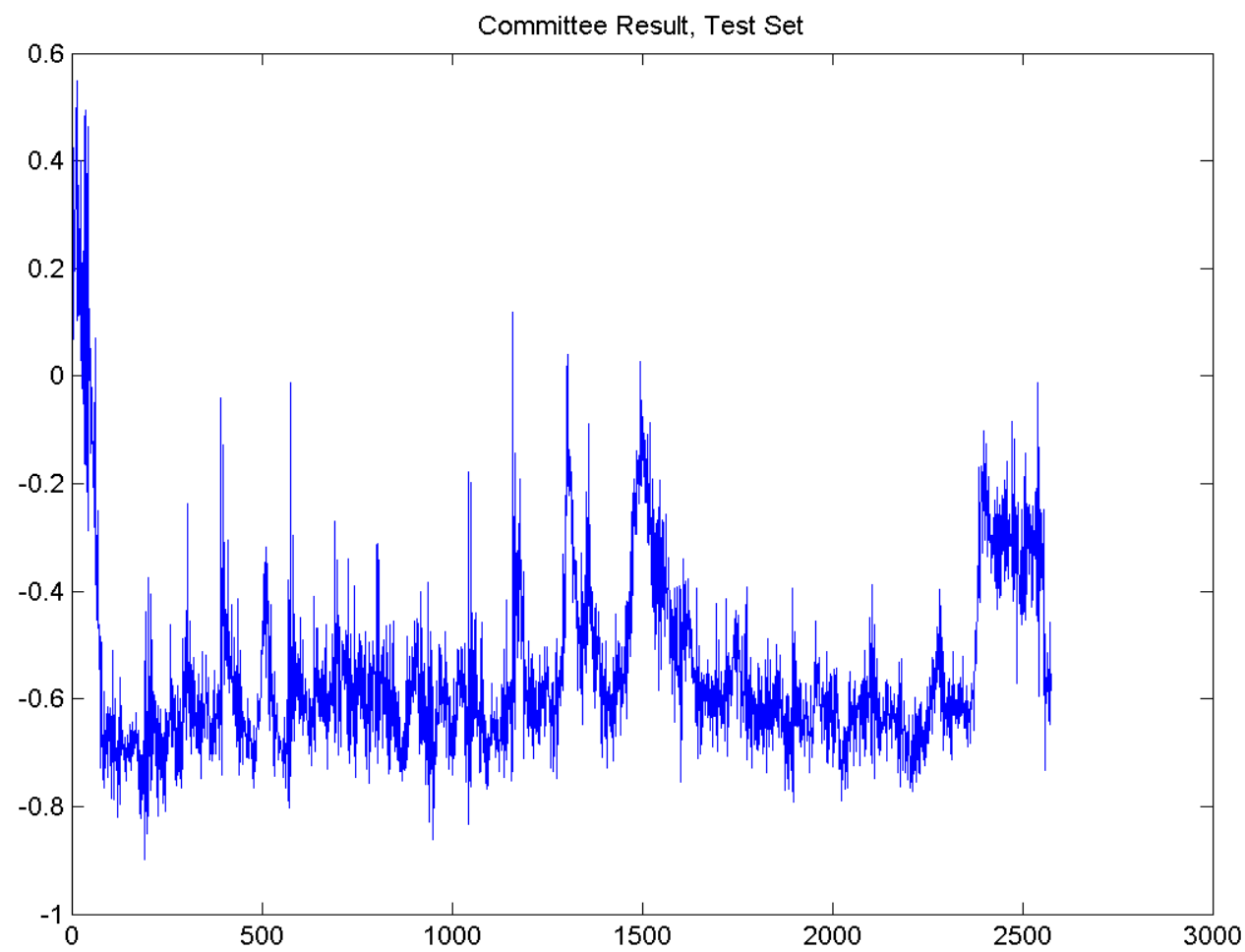

Figure 92 Sample spoof test data output, committee of last generation networks. 


\section{Discussion}

In this example, GETnet showed that it could arrive at a succinct network that not only classifies, but also performs feature extraction by accepting the raw fingerprint ridge signals and creating an internal representation through a recurrent time delay network of four neurons. The assigned task was detection of live finger perspiration temporal pattern in order to separate live fingerprints from the nonliving.

The network was evolved on less that $10 \%$ of 16 training fingerprints ridge signals. The fact that GETnet could create a reasonably accurate classifier using this scarce amount of training data confirms the fact that even without Prune, the temporal MDL and validation-based fitness score assessment mechanisms of GETnet can form a minimal, robust, and fast solution (see figures 82 and 83 ). The compactness of the solution can partially be credited to the ability of GETnet to evolve recurrent structures. The effect of recurrence can also be seen in the short transient time of the outputs, especially for the nonliving samples.

The other observation is that even though during the course of evolution the number of parallel branches was reduced, the evolved network could do well with the original 4 nodes and thus kept that number of nodes. This confirms the usefulness of the heuristic used for initialization of first generation by Genesis, so that evolution can find a suitable answer with fewer generations by having its starting point placed close to an optimum in the search space. It is interesting to note that two explicit long-term memory kernels, a fourth order in neuron 2 and a first order in neuron 3 have been developed.

Considering the use of less than $10 \%$ of only 16 fingerprint signal pairs and evolving for only 15 generations, the $83.3 \%$ accuracy of the resulting solution on the test data is indeed a very good performance. After reviewing the actual fingerprints, one can see that the misclassification of the 3 live and 2 nonliving samples stands to reason, since 
most of those fingerprints have bad quality (please see the dataset in the accompanying CD). Given that the teacher signals were chosen from images with better quality, the acquired classification and generalization is what one should expect. The utilized signals are also rough concatenations of individual ridge signals, which introduce a lot of noise by adding false jumps at the concatenation points. Even so, GETnet managed to arrive at a reasonable answer.

The other interesting observation is that the standard deviation of weight perturbations went up while the mean went down. This means that the weight search ellipsoids are being elongated to match the performance landscape while the size of their random search space is decreasing. The other mutation standard deviations were also reduced. The fact that the changes in these parameters are not as striking as the previous two runs on MG17 should not come as a surprise, since this simulation was conducted only for 15 generations and it has not fully converged. This can also be seen from the distance between train and test errors. One should also keep two things in mind. First, the intent of this simulation, contrary to the previous two problems, was not benchmarking but demonstrating the applicability of GETnet to complex real world applications that are considered to be hard and vague by human experts. Such problems call for application of black box approaches. Second, perspiration naturally has a high variation in its occurrences and cannot be accurately modeled with closed and tractable mathematical forms such as the ones that neural networks create. Thus, perspiration data should not be considered as a benchmarking dataset since no ideal perspiration sequence as a point of reference exist. Since GETnet showed reasonable performance even with few generations, the goal of this feasibility experiment was considered met.

As can be seen from the connection digraphs matrices, GETnet's solution besides being compact, is nonstandard and novel in terms of the known architectures. Such novel solutions are especially important for problems such as perspiration-based liveness detection where no standard starting point, neither for feature extraction nor classification, exists. 


\section{Conclusions and Future Work}

The perceived external world, i.e. the mental image of the existence as captured by the sensory inputs of an individual, is initially conveyed through a series of multidimensional time signals. Under normal circumstances and borrowing from the concept of mapping in mathematics, order can be considered as an invariant for the internal representations (images in the realm of mental) and the external world preimages. Biological nervous systems continuously adapt their image of the external world through multidimensional temporal sensory data. The massively parallel biological brain systems may take advantage the time delays for creation of memories and process signals. The internal states of functional units stored in memory structures plus the transition functions of neuronal circuits that create future states and outputs can be considered as the common ground between state space description of artificial and biological neural networks. GETnet adopts its design philosophy from the aforementioned ontology of the external world and theory of adaptive temporal neural networks. GETnet is an attempt by the author to address some of the most important issues among many complexities of the design and implementation of general, temporal intelligent systems by an automated and adaptive framework that requires very little human supervision and meddling. GETnet uses an elitist, preservative, static evolutionary search on top of its LMS neighborhood search. Given enough time, the evolutionary search is guaranteed to converge asymptotically to a global optimum ${ }^{124}$.

Based on what was told about GETnet in this document, one can summarize its main characteristics as

1. Generality

2. Convergence

3. Adaptive architecture

4. Finding novel answers

5. Requiring minimum human intervention. 
6. Promising initial results on single and multidimensional sequence analysis.

7. Inventing memory structures with appropriate depth and placement.

8. Minimal model variance which is especially important for small training sets.

9. New pragmatic temporal MDL for regularization.

10. Accelerated hybrid learning with Baldwin effect.

11. Can readily be parallelized.

GETnet is arguably more comprehensive and flexible compared to the other temporal neural networks. Based on the memory kernels discussed in B3-2 and B3-4, different temporal neural network architectures have been suggested. The most notable temporal designs include:

1. Time Delay Neural Networks (TDNN), introduced by Waibel et al for speech processing $^{22}$.

2. Finite Impulse Response Neural Networks (FIRnet), introduced by $\mathrm{Wan}^{23}$.

3. Elman ${ }^{24}$ and Jordan ${ }^{25}$ recurrent networks, named after their inventors.

4. Pipelined Recurrent Neural Networks (PRNN) introduced by Haykin and $\mathrm{Li}^{26}$.

5. Nonlinear autoregressive moving average (NARMA) neural networks explored by Narendra $^{27}$ for control systems.

A short comparison of the above temporal neural networks with GETnet is given blow.

TDNN is a feed forward structure with input-focused, finite, and predetermined delay line STM (see section B3-4 and figure 17). One major problem with TDNN is the fact that the best length of the input sliding window is problem-dependent and generally unknown. TDNN needs human expertise for the length of the input delay line and the general structure of the static network that comes after it. In essence, TDNN just takes a snapshot of the input sequence at each time step and from there on the system is static. TDNN lacks the infinite memory retention of recurrent memory kernels and its delay lines can only be found at the input stage. By contrast, GETnet uses more complex and 
versatile distributed memory structures that can include recurrent sub-circuits. Moreover, GETnet's memory and network structures emerge automatically through evolution.

FIRnet is the first multi-layered temporal neural network to officially implement distributed memory and thus can be called a Distributed Time-Lagged Neural Network (DTLNN). Variations of this theme, like Day and Davenport's version ${ }^{125}$ with adaptable time delays, also exist. However, FIRnet is strictly feed forward and uses only finite, predetermined delay lines. Thus, even though more versatile than TDNN, it suffers from the same lack of feedback delay loop LTM and problem of STM depth selection. The number of nodes and layers of FIRnet should also be guessed by its designer. As described earlier, GETnet does not suffer from the mentioned limitations.

Jordan, and shortly after Elman, proposed simple recurrent kernels to retain context and output activities (see figure 20). However, compared to GETnet, these networks have the following shortcomings:

- They only have recurrent memory kernels which have lower resolution.

- The recurrent connections, in the original version, are non-adaptable.

- Recurrent connections only have single step delays.

- The recurrence is restricted to the context units.

- The overall architecture needs human expert design.

PRNN is made of a layer of recurrent neurons followed by a linear tapped delay line for prediction of non-stationary time series. However, compared to GETnet, PRNN has the following limitations and disadvantages:

- The recurrent modules only have single-step delays in feedback (first order), whereas in GETnet this limitation does not exist.

- The architecture is predetermined and non-dynamic, compared to GETnet's evolutionary adaptive architecture. 
- PRNN suffers from the "baby sitting" problem since its following fundamental parameters need to be guessed by an experienced human designer:

- Number of recurrent modules.

- Number of neurons per each recurrent module.

- Number of taps in the nonlinear adaptive filter.

- Proper sample size for pre-training, since inadequate initial weights may cause divergence.

The NARMA models, as studied by Narendra, can be considered as simpler cases of GETnet networks that have feed forward layers with no internal memory structs and delay lines only at input and output layers, and with recurrent connections only from output to input. In this light, GETnet is a more general, powerful, and complex superset of NARMA model. GETnet also offers many more features within its framework, including fully automated architecture design and training. Even under the restricted NARMA configuration, GETnet offers clear advantages through its evolutionary determination of input window size, feedback delay depth, and network size and structure as well as hybrid training of connection weights. Please note that in his original work ${ }^{27}$ Narendra uses parallel-series implementation, i.e. the fed-back outputs are not from the NARMA neural network output but the teacher signal output values. Thus the mentioned neural network is not really recurrent, compared to the full recurrency of GETnet.

For future enhancement, parallel implementation will arguably have the greatest impact. First because GETnet can easily be ported into clusters and multi-processors for parallel processing, in which case its time complexity will reduces linearly (and possibly superlinearly based on locality of the code) by the number of parallel nodes. The only parts of data that need to be shared and communicated are genotypes and small synchronization messages. These inter-node communications are very manageable in size and can easily be sent over a say 100 base-T Ethernet backbone. Second, due to object-oriented design of GETnet using Matlab's neural network toolbox, there are many 
more useful parameters that are already implemented in the genotype of GETnet's network objects but are treated as constants since their inclusion into the evolution search space slows down GETnet. These parameters include different learning algorithms and their parameters, activation functions, and so on. By activating all those parameters, GETnet can further learn how to learn and become even more versatile, which is not very practical unless a parallel implementation is used.

It was also observed that based on GETnet's aggressive MDL and pruning, model variance in the evolved networks is so minimized that the solutions may not need extra validation sets for the final full training. Using all the data for training is especially beneficial for scarce training data. This is similar to biological intelligent organisms that are able to generalize using very small data sets using their intuitions or inherited model of the external world. Temporal MDL also creates fast networks, which is considered to be another sign of intelligence.

We also observed the emergence of the Baldwin effect in GETnet. This should not come as a surprise, since the first phase of the Baldwin effect is implemented by genetic transmission of structural modifications followed by partial local training through SCG. The second phase is carried out by the (noisy) best weight transfer. This is another way of describing Lamarckian evolution in weight space. The Baldwin effect accelerates the evolution towards the desired goal and avoids relying on the global but very slow phylogenetic evolutionary search, which sometimes can be similar to finding "a needle in a haystack".

Simulations showed that during the course of evolution, the radius random search always decreases. This effect can be compared to simulated-annealing. The interesting point is that this behavior emerged through evolution and was not coded into GETnet. This is a good example of how GETnet as a general intelligent system can learn the learning methodology itself. One can also expect that in case of changing environment (changing input-target data sets) this versatility may allow for more stochastic search if 
the dynamism gives this type of learning a better advantage compared to the gradient descent.

Besides parallel implementation and expansion of evolutionary search space that will lead to improvement of GETnet by allowing extra plasticity, one other suggested evolution enhancement could be avoiding the possible problem of a dominating super individual in the evolving population of solutions. This can be achieved through parent selection policy or injecting a small number of random individuals into the parent pool (immigration policy). This should create more statistical diversity in the evolved answers and also make the results of a committee of diverse solutions more robust and accurate for the unseen data. Another course of action is limiting the lifespan of each individual using a $\operatorname{EA}(\mu, \kappa, \lambda)$ evolution scheme, as described earlier in the section B4-1.

The last but not the least, it would be interesting and essential to solve more real world problems with GETnet after this feasibility phase. There are plethoras of different problems that are readily in an ideal form for GETnet. One such problem is protein secondary structure detection and similar problems in Bioinformatics. For secondary structure analysis, one needs to identify (predict) 3 alphabet strings (helix, strand, and coil secondary structures) from 20 alphabet strings (amino acids) ${ }^{126}$. As one can see, the problem is already in form of sequence prediction. The required mapping is complex and long-term dependencies may exist ${ }^{127}$.

The future applications can also explore field of biomedical signal analysis. For instance, one may be interested in finding a robust and compact real time system that can monitor one or multi-channel ECGs and detect the onset of an abnormal cardiac activity.

In conclusion, based on the very general format of GETnet's inputs and outputs, provided the availability of the required computing power, one can find novel answers to many problems. However, it must be mentioned that black box methods such as GETnet should be only utilized where good, examined classical solutions do not exist. 


\section{Appendix A: More on Gradient Conjugate Methods}

There are different methods for calculating $\alpha$ in conjugate gradient method, including:

Fletcher-Reeves,

$$
\alpha(n)=\frac{\vec{\nabla} J(n) \cdot \vec{\nabla} J(n)}{\vec{\nabla} J(n-1) \cdot \vec{\nabla} J(n-1)}
$$

Polak-Ribiere,

$$
\alpha(n)=\frac{(\vec{\nabla} J(n)-\vec{\nabla} J(n-1)) \cdot \vec{\nabla} J(n)}{\vec{\nabla} J(n-1) \cdot \vec{\nabla} J(n-1)}
$$

and Hestenes-Steifel:

$$
\alpha(n)=\frac{(\vec{\nabla} J(n)-\vec{\nabla} J(n-1)) \cdot \vec{\nabla} J(n)}{\vec{\nabla} J(n-1) \cdot \vec{\nabla} s(n-1)}
$$

The above formulas are convergent and equal for quadratic error surfaces. There are two other methods, direct search and the scaled conjugate gradient method (SCG) that are convergent for non-quadratic error surfaces as well ${ }^{128}$. There are similarities between conjugate gradient and momentum learning methods. However they differ because $\alpha$ is adaptive in case of conjugate gradient. Based on its generality and power, SCG is the method of choice for GETnet. Please see section C for more explanations. 


\section{Appendix B: Nguyen-Widrow Weight Initialization Algorithm}

GETnet uses Nguyen-Widrow method to initialize network weights in order to achieve higher training speeds. This method is implemented in Matlab's neural network toolbox v. 4. Considering the connection weight $w_{i j}$ from node $\mathrm{j}$ to node $\mathrm{i}$, the algorithm first initializes network weights $w_{i j}$ randomly between -0.5 and 0.5 . Then, the weights are initialized again according to the following formula

$$
w_{i j}=\frac{0.7 \sqrt[n_{i}]{n_{h}} w_{i j}}{\sqrt{\sum_{n=1}^{n_{h}} w_{i j}^{2}}}
$$

where $n_{i}$ is the number of nodes in the input layer and $n_{h}$ is the number of neurons in the hidden layer. The bias for each neuron, say the $\mathrm{i}^{\text {th }}$, is then set randomly between $w_{i j}$ and $-w_{i j}$. 


\section{REFERENCES}

1 Roy A. (2003), "Neural Networks: How do we make a widely used technology out of it?” IEEE NNS Connections, Vol. 1, No. 2, pp. 8-12.

2 Medina J., and Mauk M. (2000), “Computer Simulation of Cerebellar Information processing,” Nature Neuroscience Supplement, Vol. 3, November, pp. 1205-1211. 3 Voogd J,, and Glickstein M. (1998), “The Anatomy of the Cerebellum,” Trends Neuroscience 21:370-375.

4 Eccles J. C., Ito M., and Szentágothai, J. (1967), The Cerebellum as a Neuronal Machine, Springer, Berlin, New York.

5 Ito, M. (1984), The Cerebellum and Neural Control, Raven, New York.

6 Principe, J. (1994). “An Analysis of the Gamma Memory in Dynamic Neural

Networks.” IEEE Trans. on Neural Networks, 5 (2), 331-337.

7 Bailey C. H., Kandel E. R., "Structural Changes Accompanying Memory Storage," Ann Rev Physiol 55:397-426,1993.

8 Bailey, C. H., and Kandel, E. R. (1994), "Structural Changes Underlying Long-term Memory Storage in Aplysia: A Molecular Perspective," Seminars Neuroscience. 6, 35 44.

9 Genisman, Y., deToledo Morrell F., Heller R. E., Rossi M., and Parshall, R. F. (1993), "Structural Synaptic Correlate to Long-term Potentiation: Formation of Axospinous Synapses with Multiple, Completely Partitioned Transmission Zones," Hippocampus 3 (4), 435-445.

10 Jessel, T. M., and Kandel, E. R. (1993), “Synaptic Transmission: A Bidirectional and Self Modifiable form of Cell-Cell Communication," Cell Supplement, 1-30.

11 Nicoll, R. A., and Malenka, R. C. (1995), "Contrasting Two Forms of LTP in the Hippocampus," Nature 377, 115-118. 
12 Villa, A., Tsien, R. W., and Scheller, R. H. (1995), "Presynaptic Component of Longterm Potentiation Visualized at Individual Hippocampal Synapses," Science 268, 16241628.

13 Feany, M. B., and Quinn, W. G. (1995), “A Neuropeptide Gene Defined by the Drosophila Memory Mutant Amnesiac,” Science 268, 869-873.

14 Quinn W.G., Sziber P.P., Booker R. (1979), “Drosophila Memory Mutant Amnesiac,” Nature 277:212-4.

15 de Belle J. S., and Heisenberg, M. (1995), “Genetic, Neuroanatomical and Behavioral Analyses of the Mushroom Body Miniature Gene in Drosophila Melanogaster," J Neurogenet 10:24-30.

16 Bouhouche, A., and Vaysse, G. (1991), "Behavioral Habituation of the Proboscis Extension Reflex in Drosophila Melanogaster: Effect of the no Bridge,” J. Neurogenet. 7, 117-128.

17 Broadie, K., and Bate, M. (1995), “The Drosphila NMJ: A Genetic Model System for Synapse Formation and Function,” Sem. Dev. Biol. 6, 221-231.

18 Broadie, K. (1994), "Synaptogenesis in Drosophila: Coupling Genetics and Electrophysiology,” J. Physiology 88, 123-139.

19 Seidl D.R. and Lorenz D., (1991) “A Structure by Which a Recurrent Neural Network can Approximate a Nonlinear Dynamic System,” Proc. Int. Joint Conf. Neural Networks, Vol. 2, pp. 709-714.

20 Siegelmann H. T. and Sontag E. D., (1995) "On the Computational Power of Neural Networks,” J. Comput. Syst. Sci., vol. 50, no. 1, pp. 132-150.

21 Siegelmann, H.T., Horne, B.G., Giles, C.L. (1997), “Computational Capabilities of Recurrent NARX Neural Networks" Systems, IEEE Transactions on Man and Cybernetics, Part B, Vol.27, Issue 2, pp 208-215.

22 Waibel, A.; Hanazawa, T.; Hinton, G.; Shikano, K.; Lang, K.J. (1989) "Phoneme Recognition Using Time-Delay Neural Networks" [see also IEEE Transactions on Signal Processing], IEEE Transactions on Acoustics, Speech, and Signal Processing, Vol.37, Issue 3. pp 328-339. 
23 Wan E., (1993) "Time Series Prediction Using a Neural Network with Embedded Tapped Delay-Lines", in Predicting the Future and Understanding the Past, SFI Studies in the Science of Complexity, Eds. A. Weigend, N. Gershenfeld Addison-Wesley. 24 Elman, J (1990) “Finding Structure in Time, ”Cognitive Science, 14, pp 179-211. 25 Jordan, M. (1986) "Serial order: A Parallel Distributed Processing Approach," Institute for Cognitive Science Report 8604. University of California, San Diego. 26 Haykin, S. and Liang Li (1995) "Nonlinear Adaptive Prediction of Nonstationary Signals," IEEE Transactions on Signal Processing, [see also Acoustics, Speech, and Signal Processing, IEEE Transactions on], Vol.43, Issue 2, pp 526-535.

27 Narendra, K.S.and Parthasarathy, K. (1990). "Identification and Control of Dynamical Systems Using Neural Networks" IEEE Transactions on Neural Networks, Vol.1, No.1, pp 4-27.

28 Duda R.O.and Hart P.E. (1973), Pattern Classification and Scene Analysis, New York, John Wiley and Sons.

29 Fukunaga K. (1990), Introduction to Statistical Pattern Recognition. Second edition, New York: Academic Press.

30 Cover T. (1965) "Geometrical and Statistical Properties of Systems of Linear Inequalities with Applications in Pattern Recognition," IEEE Transaction on Electronic Computers, pp. 326-334, 1965.

31 Trunk G. (1979) "A Problem of Dimensionality: A Simple Example," IEEE Transactions on Pattern Analysis and Machine Intelligence vol 1, No 3, pp 306-307. 32 Vaptnik, L (1995) The Nature of Statistical Learning Theory, Springer Verlag. 33 Vaptnik, L (1998) Statistical Learning Theory, Wiley.

34 Anlauf J.K. and Biehl M. (1989) "The AdaTron: an adaptive perceptron algorithm," Europhys. Letter., 10(7) pp 687-692.

35 Lang K. J. and Witbrock M. J. (1988)"Learning to tell two spirals apart," in Proceedings of Connectionist Models Summer School, Morgan Kaufmann. 36 Poggio, T. and Girosi, F. (1990) "Networks for approximation and learning," Proceedings IEEE vol 78, No 9 pp 1481-1497. 
37 Greenstein-Messica A.and Ruppin E. (1998), "Synaptic Runaway in Associative Networks and the Pathogenesis of Schizophrenia." Neural Computation, 10(2), 451-465. 38 Aharonov R., Segev L., Meilijson I., and Ruppin E. (2003) "Localization of Function Via Lesion Analysis.” Neural Computation, 15(4), pp 885-913.

39 Kohonen, T. (1982). "Self-organized formation of topologically correct feature maps," Biological Cybernetics, 43:59 - 69.

40 Weinrich M, Sutton G, Reggia J and D'Autrechy C. (1994) "Adaptation of NonCompetitive and Competitive Neural Networks to Focal Lesions," J. Artificial Neural Networks, 1, pp 51-60.

41 Corne D, et al (2003), "The Good of the Many Outweighs the Good of the One: Evolutionary Multi-Objective Optimization." IEEE NNS Connections, Vol. 1, No. 1, pp. 9-13.

42 Orr G. and Muller K., (1998). "Neural Networks: Tricks of the Trade," Lecture notes in computer science, vol. 1524. Springer Verlag, New York.

43 Oja E., (1995) "Principal Component Analysis," in The Hand Book of Brain Theory and Neural Networks, M. A. Arbib, Ed. Cambridge, Massachusetts: The MIT press. 44 Szu H. and Hwang W. (2003), "Self Supervised Backpropagation in Stem Cells." IEEE NNS Connections, Vol. 1, No. 3, pp. 8-9.

45 Eric W. Weisstein. "Sampling Theorem." From MathWorld--A Wolfram Web Resource. http://mathworld.wolfram.com/SamplingTheorem.html 46 S. Haykin (1995), Adaptive Filter Theory, 3rd Edition. Prentice Hall. 47 Levine D.S. (1991), Introduction to Neural and Cognitive Modeling, Lawrence Erlbaum Associates, Publishers.

48 Principe J., Euliano N., and Lefebvre W. (2000) "Neural and Adaptive Systems: Fundamentals Through Simulations." Wiley.

49 Kurkova V. (1995) “Kolmogorov's theorem,” In Michael A. Arbib, editor, The Handbook of Brain Theory and Neural Networks, pp 501-502. MIT Press, Cambridge, Massachusetts.

50 Werbos P. J. (1974) "Beyond Regression: New Tools for Prediction and Analysis in the Behavioral Sciences," PhD thesis, Harvard University. 
51 Almeida, L., Langlois T., and Amaral, J. (1997) “On-line Step Size Adaptation,” Technical Report, INESC RT07/97.

52 Fahlman, S. E. (1988) "Faster-Learning Variations on Back-Propagation: An Empirical Study" in Proceedings, Connectionist Models Summer School, MorganKaufmann, Los Altos CA.

53 Kirkpatrick S., Gelatt Jr. C. D., and Vecchi M. P. (1983), "Optimization by Simulated Annealing", Science, Vol. 220, No. 4598, pp. 671-680.

54 Demuth H. and Beale M., Neural Network TOOLBOX User's Guide for use with MATLAB. The MathWorks Inc.

55 Nguyen D., and Widrow, B (1990), “ Improving the Learning Speed of the 2-Layer Neural Networks by Choosing Initial Values of Adapting Weights," in Proceedings of the International Joint Conference on Neural Networks, Vol. 3, pp. 21-26, San Diego, CA. 56 Haykin S. (1999), Neural Networks, A Comprehensive Foundation, 2nd Edition, Prentice Hall.

57 Weisstein, Eric W. "Taylor Series." Eric Weisstein's World of Mathematics. http://mathworld.wolfram.com/TaylorSeries.html 58 Barrett, R. et al (1994) "Templates for the Solution of Linear Systems: Building Blocks for Iterative Methods,” 2nd Edition, SIAM: Philadelphia, PA.

59 Luenberger D. (1986). Linear and Nonlinear programming, Addison-Wesley, Reading MA.

60 LeCun Y., Denker J. S., and Solla S. A. (1990) “Optimal Brain Damage.” In D. S. Touretzky, editor, Advances in Neural Information Processing Systems 2, pp 598-605. Morgan Kaufmann, San Mateo, CA.

61 Perrone M.P., and Cooper L.N. (1993) "When Networks Disagree: Ensemble Methods for Hybrid Neural Networks," Neural Networks for Speech and Image Processing. R.J. Mammone, editors, Chapman-Hall.

62 Guigon, A., and Burnod Y. (1995) "Short Term Memory," The Handbook of Brain Theory and Neural Networks, Arbib M. editor, MIT Press, Cambridge, MA. 
63 Wang Z-O (1996) "A Bidirectional Associative Aemory Based on Optimal Linear Associative Memory" IEEE Transactions on Computers, Volume: 45 , Issue 10. pp 11711179.

64 Oppenheim, A. and Willsky, A. (1983), Signals and Systems, Prentice Hall, Englewood Cliffs, NJ.

65 Werbos, P.J. (1990)."Backpropagation Through Time: What It Does and How to Do It." Proceedings of the IEEE, Vol.78, Issue, pp1550-1560.

66 Sandberg, I. W. and Xu L. (1997) "Uniform Approximation of Multidimensional Myopic Maps." IEEE Transactions on Circuits and Systems I: Fundamental Theory and Applications [see also IEEE Transactions on Circuits and Systems I: Regular Papers], Vol.44, Issue6, pp 477-500

67 Freeman W. J., Yao Y., and Burke B. (1988) "Central Pattern Generation and Recognizing in Olfactory Bulb: A Correlation Learning Rule," Neural Networks. Vol. 1. pp. 277-288.

68 Derakhshani R, Schuckers SAC, "Continuous Time Delay Neural Networks for Detection of Temporal Patterns in Signals." Proceedings of the IEEE 2004 International Joint Conference on Neural Networks, Budapest, Hungary.

69 Day S. P. and Davenport M. R., (1993) "Continuous-time Temporal Backpropagation with Adaptable Time Delays," IEEE Trans. Neural Networks, vol. 4, pp. 348-354. 70 Murray R. M., Li, Z. X., and Sastry S. S. (1994). A Mathematical Introduction to Robotic Manipulation. CRC Press.

71 Vidyasagar M.(1993), Nonlinear Systems Analysis, Prentice-Hall, NJ.

72 Wang X., and Blum E. K. (1995) "Dynamics and Bifurcation of Neural Networks", The handbook of Brain Theory and Neural Networks, Arbib M.A. editor, MIT Press. 73 Hertz J. (1995) "Computing with Attractors", The handbook of Brain Theory and Neural Networks, Arbib M.A. editor, MIT Press.

74 Goles E. (1995) "Energy Functions for Neural Networks", The handbook of Brain Theory and Neural Networks, Arbib M.A. Ed., MIT Press. 75 Vidyasagar M.(1993), Nonlinear Systems Analysis, Prentice-Hall, NJ.

76 Khalil H. K..(1992), Nonlinear Systems, Macmillan, NY. 
77 Lakhmi C. Jain Ed., (1999) Evolution of Engineering and Information Systems and their Applications, CRC Press.

78 Bäck T., Hammel U., and Schwefel H.P. (1997) "Evolutionary Computation:

Comments on the History and Current State,'IEEE Trans. On Evolutionary Computation, Vol. 1, No.1.

79 J. Heitkoetter J., and Beasley D. (2001), The Hitch-Hiker's Guide to Evolutionary Computation (FAQ for comp.ai.genetic). online: http://www.faqs.org/faqs/ai-faq/genetic/ 80 Bäck T. (1995) "Evolution Strategies: An Alternative Evolutionary Algorithm," Artificial Evolution: European Conference, Ae 95 Brest, France, Selected Papers Lecture Notes in Computer Science; Alliot J.-M., Lutton E., Schoenauer M., and Ronald E. editors, Vol 1063, pp. 3-20.

81 Fogel D.B., and Fogel L.J. (1995) “An Introduction to Evolutionary Programming," Artificial Evolution: European Conference, Ae 95 Brest, France, Selected Papers Lecture Notes in Computer Science; Alliot J.-M., Lutton E., Schoenauer M., and Ronald E. editors, Vol 1063, pp. 21-33.

82 Fogel L.J. (1999), Intelligence Through Simulated Evolution: Forty Years of Evolutionary Programming, Wiley Series on Intelligent Systems.

83 Bäck T., Rudolph G., and Schwefel H.-P.,(1992)"Evolutionary Programming and Evolution Strategies: Similarities and Differences," Fogel D.B., and Atmar W.editors, pp 11-22, IEEE Proceedings of the Second Annual Conference on Evolutionary Programming, La Jolla, CA, USA.

84 Sipper M. (1995) “An Introduction to Artificial Life”, Explorations in Artificial Life (special issue of AI Expert), pp. 4-8, September Issue, Miller Freeman.

85 Rudolph, G. (1994) "Convergence Analysis of Canonical Genetic Algorithms". IEEE Trans. on Neural Networks, special issue on Evolutionary Computation, Vol. 5, No. 1, pp 96-101.

86 Schwefel H. P. (1981). Numerical Optimization of Computer Models, John Wiley \& Sons, New York.

87 Nolfi, S., and Parisi, D. (1995) "Genotypes for Neural Networks", The handbook of Brain Theory and Neural Networks, Arbib M.A. editor., MIT Press pp. 431-434. 
88 Yao, X. (1999), “Evolving Artificial Neural Networks,” Proceedings of IEEE, September, 87 (9) pp. 1423-1447.

89 Whitley, D. (1995) “Genetic Algorithms and Neural Networks” in Genetic Algorithms in Engineering and Computer Science, Periaux J., Galan M., and Cuesta P. editors, John Wiley, pp. 203-216.

90 Grönroos, M. (1999) “A Comparison of Some Methods for Evolving Neural Networks" Proceedings of GECCO'99, Vol 2. Morgan Kaufmann Publishers, San Francisco, California.

91 Yao, X. and Lio Y (1997). “A New Evolutionary System for Evolving Artificial Neural Networks.” IEEE Transactions on Neural Networks, Vol 8 No 3 pp 694-713. 92 Bengio Y., Simard P., and Frasconi P., (1994) "Learning Long-term Dependencies with Gradient is Difficult," IEEE Trans. Neural Networks, vol. 5, pp. 157-166.

93 Frasconi P., Gori M., and Soda G., (1992) “Local Feedback Multilayered Networks," Neural Computation, vol. 4, pp. 120-130.

94 Gori M., Maggini M., and Soda G. (1994), "Scheduling of Modular Architectures for Inductive Inference of Regular Grammars,” Proc. ECAI'94 Workshop, Combining Symbolic Connectionist Procedures, Amsterdam, The Netherlands, pp. 78-87.

95 El Hihi S. and Bengio Y., (1996) "Hierarchical recurrent neural networks for longterm dependencies," Neural Information Processing Systems 8. Cambridge, MA: MIT Press.

96 Hochreiter S. and Schmidhuber J. (1995) "Long Short-term Memory," Forschungsberichte Künstliche Intelligenz FKI-207-95, Germany: Institut für Informatik, Technische Universität München.

97 Baldwin, J. M. (1896) "A New Factor in Evolution." American Naturalist 30: 441451. Reprinted in Adaptive Individuals in Evolving Populations: Models and Algorithms, edited by R. K. Belew and M. Mitchell (SFI Studies in the Sciences of Complexity, Proc. Vol. XXVI, Addison-Wesley, Reading, MA, 1996).

98 Hinton, G. E. and Nowlan, S. J. (1987) "How Learning can Guide Evolution," Complex Systems, 1, 495-502. 
99 Jim K., Giles C.L., and Horne B.G. (1996) "An Analysis of Noise in Recurrent Neural Networks: Convergence and Generalization", IEEE Trans. Neural Networks, Vol. 7, No. 6, pp. 1424-1439.

100 Fletcher, R. (1975). Practical Methods of Optimization, John Wiley \& Sons.

101 Gill, P.E., W. Murray, M.H. Wright (1980). Practical Optimization, Academic Press. 102 Hestenes, M. (1980). Conjugate Direction Methods in Optimization, Springer Verlag, New York.

103 Powell, M. (1977). Restart Procedures for the Conjugate Gradient Method, in: Mathematical Programming, pp 241-254.

104 Moller M. (1993) “A Scaled Conjugate Gradient Algorithm for Fast Supervised Learning," Neural Networks, 6:525-533.

105 Sathyanarayan S. R., and Kumar C. (1996) "Evolving Recurrent Bilinear Perceptrons for Time Series Prediction", ASME Press Series on Intelligent Engineering Systems through Artificial Neural Networks ANNIE-1996 Proceedings, St. Louis, Missouri. 106 Kurzweil R (2000), The Age of Spiritual Machines: When Computers Exceed Human Intelligence, Penguin.

107 Box, G.E.P., Jenkins G.M., and Reinsel G.C. (1994), Time Series Analysis: Forecasting and Control, Third edition, Prentice Hall.

108 Chechik G., Meilijson I., Ruppin E. (1998), "Synaptic Pruning in Development: A Computational Account." Neural Computation, 10(7), 1759-1777.

109 Prinicipe J, Rathie A., and Kuo J.M. (1992) "Prediction of Chaotic Time Series with Neural Networks and the Issue of Dynamic Modeling", International Journal of Bifurcation and Chaos, Vol. 2, pp. 989-996.

110 Yao X. and Liu Y. (1997), "EPNet for chaotic time-series prediction," in Selected Papers from the Frist Asia-Pacific Conference on Simulated Evolution and Learning SEAL'96, X. Yao, J.-H. Kim, and T. Furuhashi, editors, Vol. 1285 of Lecture Notes in Artificial Intelligence, pp. 146-156, Springer-Verlag, Berlin.

111 De Falco A. et all (1998). “Optimizing Neural Networks for Time Series Prediction.” Third World Conference on Soft Computing WSC3.

112 http://neural.cs.nthu.edu.tw/jang/benchmark/ 
113 Mackey, M. C., and Glass, L. (1977) "Oscillation and Chaos in Physiological Control Systems," Science: 197, 287-289.

114 de Menezes M. A., and dos Santos R. M. Z. (2000). "The Onset of Mackey-Glass Leukemia at the Edge of Chaos," International Journal of Modern Physics C, Vol. 11, No. 8.

115 http://www.cse.ogi.edu/ ericwan/data.html

116 http://www.cse.ogi.edu/ ericwan/data.html

117 Schuckers S. A. C. (2002) "Spoofing and anti-spoofing measures," Information Security Technical Report, Vol. 7, No. 4, pages 56-62,.

118 Matsumoto T., Matsumoto H., Yamada K., and Hoshino S. (2002) "Impact of Artificial 'gummy' Fingers on Fingerprint Systems”, Proceedings of SPIE, vol. 4677. 119 van der Putte T., and Keuning J. (2000), "Biometrical Fingerprint Recognition: Don't Get Your Fingers Burned," in Proceedings of the Fourth Working Conference on Smart Card Research and Advanced Applications, Kluwer Academic Publishers, pp. 289303.

120 Derakhshani R, Schuckers S. A. C., Hornak L, and O’Gorman L (2003) "Determination of Vitality from A Non-Invasive Biomedical Measurement for Use in Fingerprint Scanners.” Pattern Recognition Journal, Vol. 36, No.2, pp. 383-396.

121 Schuckers S. A. C., Derakhshani R, Parthasaradhi S, and Hornak L (2004) "Improvement of an Algorithm for Recognition of Liveness using Perspiration in Fingerprint Devices.” Proceedings of SPIE, Vol. 5404.

122 Schuckers S. A. C., Parthasaradhi S., Derakhshani R., Hornak L. (2004) "Comparison of Classification Methods for Time-Series Detection of Perspiration as a Liveness Test in Fingerprint Devices." To appear in the proceedings of the International Conference on Biometric Authentication, Hong Kong (Springer-Verlag LNCS series). 123 Derakhshani R. (1999), "Determination of Vitality from a Non-invasive Biomedical Measurement for use in Integrated Biometric Devices.” Master's Thesis, West Virginia University, https://etd.wvu.edu/etd/etdDocumentData.jsp?jsp_etdId=1035 124 Fogel D.B. (1995), Evolutionary Computation: Toward a New Philosophy of Machine Intelligence, IEEE Press, NY. 
125 Day, S. and M. Davenport (1993),’Continuous-time Temporal Back-propagation with Adaptable Time Delays," IEEE Transactions on Neural Networks, Vol. 4 No. 2 pp. 348-354.

126 Rost B, and Sanders C., (1995) "Protein Structure Prediction", The handbook of Brain Theory and Neural Networks, Arbib M.A. editor, MIT Press.

127 Baldi P., Brunak S., Frasconi P., Pollastri G., and Soda G., (1999) "Exploiting the Past and the Future in Protein Secondary Structure Prediction", Bioinformatics, 15, 937946.

128 Shepherd, A. (1997) Second-Order Methods for Neural Networks: Fast and Reliable Training Methods for Multi-Layer Perceptrons, Springer Verlag. 


\section{REZA DERAKHSHANi \\ CURRICULUM VITAE}

\section{RESEARCH INTERESTS}

Computational Intelligence and its applications in Biomedical Signal Processing, Bioinformatics, and Biometrics.

\section{EDUCATION}

\section{August 2004 (Expected)}

Ph.D. in Computer Engineering. West Virginia University, Morgantown, WV. Dissertation topic: "Biologically Inspired Temporal Evolutionary Neural Circuits.” Advisor: Dr. Stephanie A.C. Schuckers.

\section{December 1999}

M.S. in Electrical Engineering (Major: Digital Systems, Minor: Software Engineering) West Virginia University, Morgantown, WV.

\section{January 1994}

B.S. in Electrical and Electronics Engineering. Iran University of Science and Technology, Tehran, Iran.

\section{Professional Experience}

\section{A. Teaching}

January 2004 - May 2004

Adjunct Faculty, Computer Science Department, Georgetown University.

- Teaching COSC 127/COSC 506: Mathematical Methods for Computer

Science/Concrete Mathematics and Complexity.

- Teaching COSC 251 Computer Systems Fundamentals II.

January 2003 - May 2003

Adjunct Faculty, Computer Science Department, Georgetown University.

- Taught COSC 251: Computer Systems Fundamentals II. 


\section{B. Academic Research}

\section{October 1998 - May 2004}

Research Assistant, Biomedical Signal Analysis Lab (BioSAL), and NSF IUCRC Center for Identification Technology Research (CITeR), Lane Department of Computer Science and Electrical Engineering, West Virginia University.

- Research on biometric systems and their vulnerabilities, including design of novel perspiration-based liveness detection algorithms for making fingerprint scanners spoof-proof ( 2 pending patents).

- Researched on processing techniques for cardiovascular ultrasound Doppler signals and images.

- Research on new evolving temporal artificial neural networks and their applications.

- Tested and evaluated different biometric systems.

\section{Industry}

\section{May 1996 - October 1997}

Data Communication Engineer, R\&D Department, Kish Communications Industries (KCI), Tehran, Iran.

- Increased Pars-Telefonkar PBXs' modem connection speeds from 1200 to 28800 BPS through circuit correction.

- Created remote connection between corporate LANs.

- Advised KCI in technology purchasing.

- Designed/supervised several projects in KCI R\&D, including fax/voice/data switch, experimental modem, etc.

\section{April 1994 - May 1996}

Electronic Circuit Designer, Atbin Co. Tehran, Iran.

- Designed and constructed several economical internal and external EPROM and/or micro-controller programmers using three different algorithms for generic memory families.

\section{April 1994- February 1996}

Electronic Circuit Designer, Telecommunication College's Repair Department, Army Telecommunication \& Electronics Training Center (Compulsory Military Service), Tehran, Iran.

- Designed and constructed high precision and temperature compensated RF synthesizers using PLL for military communication devices.

\section{Other}

\section{May 1998 - October 1998}

Application Programmer, West Virginia University Student Affairs Business Operations (SABO).

- Created simultaneous multi-user online spreadsheets. 


\section{January 1998 - May 1998}

Home Page Developer, West Virginia NASA Space Grant Consortium.

- Created and maintained multi-media web pages for the consortium.

\section{Patents}

1. Schuckers SAC, Derakhshani $R$, Hornak L, "Liveness Detection Technique for Multi- Technology Fingerprint Sensors," Disclosure filed January 4, 2003, patent application in process.

2. O'Gorman L, Schuckers SAC, Derakhshani R, Hornak L, "Method and Apparatus for Determining a Living Finger on a Fingerprint Sensor," US Provisional Patent Application, Docket Number P-7653 US. Filed October 7, 1999.

\section{Publications}

\section{A. Peer-Reviewed Journal Papers}

1. Parthasaradhi S, Derakhshani R, Hornak L, Schuckers SAC, "Time-Series Detection of Perspiration as a Liveness Test in Fingerprint Devices." To appear on the IEEE Transactions on Systems, Man, and Cybernetics, Part C: Applications and Reviews.

2. Derakhshani R, Schuckers SAC, Hornak L, O'Gorman L, "Determination of Vitality from A Non-Invasive Biomedical Measurement for Use in Fingerprint Scanners." Pattern Recognition Journal, Vol. 36, No.2, pp. 383-396, 2003.

\section{B. Conference Proceedings}

1. Derakhshani $R$, Schuckers SAC, "Continuous Time Delay Neural Networks for Detection of Temporal Patterns in Signals." To appear on the proceedings of the IEEE 2004 International Joint Conference on Neural Networks, Budapest, Hungary.

2. Schuckers SAC, Parthasaradhi S, Derakhshani R, Hornak LA, "Comparison of Classification Methods for Time-Series Detection of Perspiration as a Liveness Test in Fingerprint Devices." To appear on the proceedings of the 2004 International Conference on Biometric Authentication, Hong Kong (Springer-Verlag LNCS series).

3. Schuckers SAC, Derakhshani R, Parthasaradhi S, Hornak LA, "Improvement of an algorithm for recognition of liveness using perspiration in fingerprint devices." Proceedings of SPIE Vol. \#5404, 2004. 
4. Schuckers SAC, Hornak LA, Derakhshani R, Parthasaradhi S, "Initial Results of Spoofing and Liveness Detection in Fingerprint Scanners." Abstract in the Proceedings of Biometrics 2002, London, UK, November 2002.

5. Schuckers SAC, Hornak L, Norman T, Derakhshani R, Parthasaradhi S, "Issues for Liveness Detection in Biometrics." Abstract in the Proceedings of the Biometrics Consortium Conference, Arlington, VA, September 2002.

6. Derakhshani $R$, Schuckers SAC, "Biologically Inspired Evolutionary Temporal Neural Circuits." Proceedings of IEEE World Congress on Computational Intelligence, Honolulu, HI, 2002

7. Derakhshani R, Schuckers SAC, Hornak L, O'Gorman L, "Neural Network-Based Approach for Detection of Liveness in Fingerprint Scanners." Proceedings of the International Conference on Artificial Intelligence, Las Vegas, NV. CSREA Press, pp. 1099-1105, 2001.

8. Derakhshani $R$, Schuckers SAC, "Determination of Vitality From A Non-Invasive Biomedical Measurement for Use in Fingerprint Scanners." Abstract in Proceedings of World Congress on Medical Physics and Biomedical Engineering, Chicago, IL, 2000.

9. Derakhshani $R$, Schuckers SAC, "Determination of Vitality From A Non-Invasive Biomedical Measurement for Use in Fingerprint Scanners." Abstract in Proceedings of International Association for Identification 85th International Educational Conference, Charleston, WV, 2000.

\section{AWARDS}

1. January 2002 - January 2004. Lane Fellowship for the highest academic achievement in the field of study. Lane Department of Computer Science and Electrical Engineering, West Virginia University.

2. May 2002 - IEEE Travel Award for 2002 World Congress on Computational Intelligence, Honolulu, HI.

\section{Memberships and Certifications}

1. West Virginia Society of Professional Engineers (Engineer in Training).

2. Canadian Society of Professional Engineers (Certified).

3. IEEE Engineering in Medicine and Biology Society (Member).

4. IEEE Neural Network Society (Member).

5. IEEE Computer Society (Member). 


\section{COMPuter KNOWLEDGE}

Languages: JAVA, Visual BASIC, C/C++, IA32 Assembly, LISP, FORTRAN.

OS: Windows, UNIX, DOS, and VMS.

Software: MATLAB/SimuLink, NeuroSolutions, PlaNet, Maple, LASI, ORCAD, Active VHDL, MicroCap, Electronic Workbench, MS Visio, Front Page, and Office.

\section{LANGUAGE SKILLS}

English: Near-native fluency.

Farsi: Native speaker. 